\title{
Gas-Solid Interaction, Flow Behavior analysis and Development of a Design Basis equation for the Dense Entry Region of an Asymmetrically Loaded Cold Flow CFB Riser
}

\author{
Rajiv Dastane \\ West Virginia University
}

Follow this and additional works at: https://researchrepository.wvu.edu/etd

\author{
Recommended Citation \\ Dastane, Rajiv, "Gas-Solid Interaction, Flow Behavior analysis and Development of a Design Basis \\ equation for the Dense Entry Region of an Asymmetrically Loaded Cold Flow CFB Riser" (2010). Graduate \\ Theses, Dissertations, and Problem Reports. 4577. \\ https://researchrepository.wvu.edu/etd/4577
}

This Thesis is protected by copyright and/or related rights. It has been brought to you by the The Research Repository @ WVU with permission from the rights-holder(s). You are free to use this Thesis in any way that is permitted by the copyright and related rights legislation that applies to your use. For other uses you must obtain permission from the rights-holder(s) directly, unless additional rights are indicated by a Creative Commons license in the record and/ or on the work itself. This Thesis has been accepted for inclusion in WVU Graduate Theses, Dissertations, and Problem Reports collection by an authorized administrator of The Research Repository @ WVU. For more information, please contact researchrepository@mail.wvu.edu. 
Gas-Solid Interaction, Flow Behavior analysis and Development of a Design basis equation for the Dense Entry Region of an Asymmetrically Loaded Cold Flow CFB Riser

\author{
Rajiv Dastane
}

\author{
Thesis submitted to the \\ College of Engineering and Mineral Resources \\ at West Virginia University \\ in partial fulfillment of the requirements \\ for the degree of
}

\author{
Master of Science \\ in \\ Aerospace Engineering \\ Eric Johnson, Ph.D., Chair \\ Bruce Kang, Ph.D. \\ Larry Shadle, Ph.D.
}

Department of Mechanical and Aerospace Engineering

Morgantown, West Virginia

2010

Keywords: Circulating Fluidized Beds, Gas Solids Flow, Riser, Tracer Gas Profile, Turbulence Modulation, MANOVA, Correlation Ratio

Copyright 2010, Rajiv Dastane 


\begin{abstract}
Gas-Solid Interaction, Flow Behavior analysis and Development of a Design basis equation for the Dense Entry Region of an Asymmetrically Loaded Cold Flow CFB Riser
\end{abstract}

\title{
Rajiv Dastane
}

The dispersion of a gas tracer was used to indicate the effectiveness of the mixing process of an injected flow of solids into the dense bed region of NETL's cold flow CFB riser in three distinctly different fluidization regimes. NETL's cold flow test facility mimics commercial scale transport reactors with side entry of solids into the vertical riser. Pure $\mathrm{CO}_{2}$ was used as the tracer gas and was introduced continuously into the injected flow of solids and it was assumed to essentially remain in the injected flow stream. The tracer gas would be released from the injected flow stream as the as the flow stream begins to disintegrate. As the stream loses its identity the remaining tracer gas would be released. The tracer gas distribution was measured using inline IR $\mathrm{CO}_{2}$ detectors across the cross-sectional area of the riser at four different elevations, two near the injection point and two further downstream. Due to the high solids hold up and high reactant concentrations, a significant portion of the reaction can take place in the dense bed region. The effectiveness of a Transport Reactor depends on its ability to adequately mix the incoming flows of reactants: fuel, sorbent and air. These reactants have to be dispersed across the reactor's cross-sectional area by the different mixing mechanisms. A good description of the flow behavior is also essential in developing and validating predictor reactor models as well as in developing crucial gas and solids mixing relationships that will can be incorporated and validated for CFD codes (MFIX). In addition there are several operational variables (independent variables) that influence this mixing behavior. Multivariable analysis of variance (MANOVA) 
model were developed for the NETL cold flow CFB riser based on the dispersion data. The mixing process as a function of the operating parameters is empirically proposed outlining the independent variables (operating and system parameters) which significantly influenced the dispersion of the tracer gas. Since the contacting between the gas and solid phases in a fluidized bed is mainly governed by the degree of gas mixing, the equations governing the gas mixing in fast-fluidized beds is useful in reactor design. The results from this work have been presented at the 2010 Multiphase Flow Workshop organized by NETL at Pittsburgh. 


\section{ACKNOWLEDGEMENTS}

I would like to thank the Department of Energy for financially supporting this work. I would like to thank my parents and God for giving me the opportunity to come to the US without which this would not have been possible. I would like to thank my advisor, Dr. Eric Johnson for his support, guidance and encouragement. I would like to thank Dr. Larry Shadle for working very closely with me and giving me the opportunity to work with the group of scientists at the Department of Energy National Energy Technology Laboratory in Morgantown, WV. It was an exceptional opportunity to work with this dynamic team and also to work on such a large scale system. I would like to thank Dr. Jim Spenik and Dr. Chris Ludlow for the Department of Energy for listening ad offering many helpful suggestions. I would also like to thank Clifford Judy for the expertise in manufacturing the injection and detection probes. I would also like to thank Dr. Jacky Prucz for having the faith in me during good and bad times. 


\section{Table of Contents}

Title

Page

1. Introduction and Literature Review 1

1.1. Background 1

1.2. Mixing in the dense entry region 4

1.3. Objectives of this study 8

1.4. Significance of this study 8

1.5. Structure of the thesis $\quad 10$

2. Experimental Facility, Instrumentation and Procedure 12

2.1.Achieving high density/high flux conditions in risers 12

2.2. Classical Choking $\quad 12$

2.2.1. Equipment induced instabilities 13

2.2.2. Experimental Apparatus 15

2.3.Instrumentation $\quad 19$

2.3.1. Air flow measurements 19

2.3.2. Differential pressure measurements 19

2.3.3. Circulation rate measurements 20

2.3.4. The test matrix 21

2.4. Region classification of the NETL cold flow CFB riser 25

2.5. The dense entry region of the riser $\quad 27$

2.6. Probe Instrumentation 28

2.6.1. Tracer gas flow rate 28

2.6.2. Tracer gas probe system 28

$\begin{array}{ll}\text { 2.6.3. Gas injection and sampling system } & 29\end{array}$

3. Background - Tracer gas study 32

3.1. Gas solids fluidization regimes $\quad 32$

3.2. Flow behavior and regime transition in a high density CFB riser $\quad 37$

3.3. Relevant contemporary work done 38

4. Gas tracer study results $\quad 64$ 
4.1.Introduction 64

4.1.1. Different regimes and average bed voidage 64

4.1.2. Fluidization regimes at the test matrix operating conditions 65

4.2. Co-flow hypothesis $\quad 67$

4.3.Tracer gas measurements $\quad 71$

4.4. Radial tracer gas dispersion profiles $\quad 73$

4.4.1. Tracer Gas Profile results 73

4.4.2. Analysis of results 76

5. Hydrodynamic analysis of gas tracer results 82

5.1.Introduction $\quad 82$

5.2. Understanding the tracer gas profiles $\quad 94$

5.2.1. Scenario 1: No turbulence modulation 96

5.2.2. Scenario 2 and 3: Presence of turbulence modulation $\quad 97$

6. Statistical Analysis of gas tracer results 100

6.1.Introduction 100

6.2. The F-Distribution 103

6.3. Definition of Terminology 105

6.4. Testing the null hypothesis 108

6.4.1. Decision making rules 110

6.5. Checking the significance 113

6.6. Effect Size, power and sample size 125

6.6.1. Descriptive measures of effect size 125

6.6.1.1. Differences relative to variability of the observations 126

6.6.1.2. The proportion of variability accounted for by an effect 128

6.7. Formulation of the design basis equation $\quad 130$

7. Overall conclusions and future work 133

7.1. Overall conclusions 133

$\begin{array}{ll}\text { 7.2. Recommendations for future work } & 135\end{array}$

8. Appendices 136

8.1. Confidence Interval and Error Estimates 137 
8.2. Determination of average tracer concentration 138

$\begin{array}{ll}\text { 8.3.The F Table } & 139\end{array}$

8.4.Primer on Statistics 140

8.5.Primer on ANOVA 143

9. References 146 


\section{List of Figures}

Figure $\quad$ Page

Figure1.1 Typical configuration of a CFB 3

Figure1.2 Elgobashi's classification of particle turbulence coupling $\quad 10$

Figure 2.1 Schematic Diagram of the NETL Cold Flow CFB 15

$\begin{array}{ll}\text { Figure 2.2 SEM image of Glass Beads } & 17\end{array}$

Figure 2.3 The spiral vane used for measuring solids circulation rate 21

Figure 2.4 Operating Regime for the $750 \mu \mathrm{m}$ Polyethylene beads 24

Figure 2.5 The test matrix points using polyethylene beads 25

Figure 2.6 The NETL Cold Flow CFB Unit $\quad 27$

Figure 2.7 Locations at which tracer gas measurements 28

Figure 2.8 Layout of the Gas Injection System 31

Figure 2.9 Sampling Probe System 32

Figure 3.1 Gas Solid Fluidization Regimes 33

Figure3.2. Comparison of prediction of onset of DSU flow regime 37

Figure 3.3 Flow structure in a Solid Gas Riser $\quad 39$

Figure3.4 Principle outline of the 12 MW boiler (Sterneus) 40

Figure3.5. Schematic outline of the measurement equipment (Sterneus) 41

Figure3.6. Gas profiles measured at three different occasions (Sterneus) 43

Figure 3.7 Gas profiles measured at three different occasions (Sterneus) 43

Figure 3.8 Gas profiles measured at three different occasions (Sterneus) 44

Figure 3.9 Experimental Set Up (Zheng) $\quad 45$

Figure 3.10 Tracer Gas System (Zheng) 45

Figure3.11 Radial Dispersion Coefficient (Zheng) 46

Figure3.12 Schematics of Experimental Apparatus (Kim) 48

Figure3.13 Schematic diagram of the momentum probe (Kim) 49

Figure3.14 Axial Profile of cross sectional mean solids holdup (Kim) 51

Figure3.15 Effect of solids mass flux on solids hold up (Kim) $\quad 52$

Figure3.16 Pilot plant CFB system Riser (Werther) 53

Figure 3.17 Experimental setup for solids mixing (Werther) 54

Figure3.18 Horizontal $\mathrm{CO}_{2}$ gas concentration profile (Werther) 56

Figure3.19 Vertical profiles of local temperature (Werther) $\quad 57$ 
Figure 3.20 Vertical profiles of local temperature (Werther)

Figure3.21. Schematic diagram of the circulating fluidized bed (Bi) 60

Figure3.22 Schematic diagram of the ozone concentration measurement system(Bi) 62

Figure3.23 Typical axial solids holdup pro6les in the riser (Bi) 64

Figure3.31 Radial distributions of ozone concentrations (Bi) 65

Figure3.25 Axial ozone concentration profiles (Bi) 66

Figure3.26. Radial Profiles of Tracer Gas Measurements (Werther) 68

Figure3.27. Upstream and downstream tracer profiles (Werther) 69

Figure3.28. Stability of Down flowing tracer gas (Werther) 69

Figure 4.1 Dense Bed Height for different operating conditions 70

Figure 4.2 Bubbling regimes in the cold CFB $\quad 72$

Figure 4.3 Determination of the bottom bed height, $\mathrm{H}_{\mathrm{x}} \mathrm{v} \quad 73$

Figure 4.4. Solids Flux at Various Inventory Levels 74

Figure 4.5. AAD as a function of superficial gas velocity $\quad 75$

Figure 4.6 AAD as a function of superficial gas velocity for the test matrix $\quad 76$

Figure 4.7 Co Flow of $\mathrm{CO}_{2}$ tracer gas and Solids $\quad 77$

Figure 4.8 Expected tracer profile for low solids momentum $\quad 78$

Figure 4.9 Expected tracer profile for intermediate solids momentum 79

Figure 4.10 Expected tracer profile for high solids momentum $\quad 79$

Figure4.11 Locations at which tracer gas measurements $\quad 80$

Figure 4.12 Schematic of the probe and injection layout $\quad 81$

Figure 4.13 Radial tracer dispersion in E/W direction for low solids feed 82

Figure 4.14 Radial tracer dispersion in E/W direction for intermediate solids feed 83

Figure 4.15 Radial tracer dispersion in E/W direction for high solids feed 83

Figure 4.16 Radial tracer dispersion in N/S direction for low solids feed 84

Figure 4.17 Radial tracer dispersion in N/S direction for intermediate solids feed 84

Figure 4.18 Radial tracer dispersion in N/S direction for high solids feed 85

Figure 5.1 Turbulence intensity of air in presence of $3.4 \mathrm{~mm}$ particles 95

Figure 5.2 Turbulence intensity of air in presence of $0.2 \mathrm{~mm}$ particles 95

Figure 5.3 Profiles of turbulence intensity of air carrying solids 98

Figure 5.4 Effect of loading and particle density on turbulent intensity 99

Figure 5.5 Fiber optic for determining the particle velocity 104

Figure 5.6 Scenario 1 Gas Phase Free Jet 105 
Figure 5.7 Scenario 2 and 3 Modulation of Turbulence 107

Figure 6.1 Random Samples being selected from a Global Set 110

Figure 6.2.The F - Distribution 113

Figure 6.3 Magnitudes of significant influences 127

Figure 6.4 Variation in magnitude of dependence on operating conditions $\quad 135$

Figure 6.5 Pie chart showing contributions to total variance 144 


\section{List of Symbols}

\begin{tabular}{|c|c|}
\hline Symbol & Description of the Symbol \\
\hline $\mathrm{H}_{1}$ & Alternate hypothesis \\
\hline $\mathrm{AAD}$ & Average absolute deviations \\
\hline$\zeta$ & Axial elevation \\
\hline$\varsigma$ & Azimuthal position \\
\hline $\mathrm{H}_{\mathrm{x}}$ & Bed height \\
\hline $\mathrm{H}_{\mathrm{mf}}$ & Bed height at minimum fluidization velocity \\
\hline $\mathrm{H}_{\mathrm{x}} / \mathrm{L}$ & Bed height to Width \\
\hline$\tau_{\mathrm{s}}$ & Characteristic dimension of the particle \\
\hline$\tau_{\mathrm{C}}$ & $\begin{array}{l}\text { Characteristic time scale of the turbulent eddies in the continuous phase } \\
\text { according to the commonly used } k-\varepsilon \text { turbulence model equation }\end{array}$ \\
\hline $\mathrm{U}_{\mathrm{ch}}$ & Choking velocity \\
\hline$\varepsilon_{\mathrm{sf}}$ & Cross-sectionally averaged solids holdup \\
\hline $\mathrm{df}$ & Degrees of freedom \\
\hline$\rho_{\text {air }}$ & Density of air \\
\hline$\rho_{\mathrm{g}}$ & Density of gas(air) \\
\hline$\rho_{\mathrm{s}}$ & Density of the solids \\
\hline$d_{p}$ & Diameter of particle \\
\hline $\mathrm{D}$ & Diameter of riser \\
\hline $\mathrm{D}_{\mathrm{x}}$ & Dispersion coefficient \\
\hline$\varepsilon_{\mathrm{TE}}$ & Dissipation of turbulent energy \\
\hline$\Delta \mathrm{P}_{\text {dist }}$ & Distributor pressure \\
\hline $\mathrm{F}$ & Experimental or calculated F ratio \\
\hline $\mathrm{f}_{\mathrm{w}}$ & Frequency of vortex shedding \\
\hline$f\left(l_{w}\right)$ & Function of the wake size \\
\hline$\sigma$ & Gas (air) phase turbulence intensity \\
\hline GB & Glass beads \\
\hline g & Gravitational constant \\
\hline $\mathrm{M}_{\mathrm{s}}^{*}$ & Mass flow rate of solids \\
\hline
\end{tabular}




\begin{tabular}{|c|c|}
\hline $\mathrm{M}_{\mathrm{s}, \mathrm{c}}$ & Mass flow rate of solids at choking \\
\hline$\mu$ & Mean \\
\hline $\mathrm{C}_{0}$ & Mean mixed concentration \\
\hline $\bar{x}$ & Mean of the time series \\
\hline MS & Mean sum of squares \\
\hline $\mathrm{U}_{\mathrm{sg}}$ & Mean superficial velocity of the gas (air) in the axial direction \\
\hline$\Delta \mathrm{P}_{\mathrm{m}}$ & Measured time-mean pressure drop \\
\hline $\mathrm{U}_{\mathrm{mb}}$ & Minimum bubbling velocity \\
\hline $\mathrm{U}_{\mathrm{mf}}$ & Minimum fluidization velocity \\
\hline a & Momentum transfer coefficient \\
\hline $\mathrm{H}_{0}$ & Null hypothesis \\
\hline$\tau_{\mathrm{v}}$ & Particle relaxation time \\
\hline$\tau_{v}$ & Particle response time \\
\hline$\alpha$ & Probability \\
\hline $\mathrm{U}_{\mathrm{pa}}$ & Purging air velocity \\
\hline C & $\begin{array}{l}\text { Ratio of mass of the dispersed phase to the carrier phase in a two-phase } \\
\text { mixture }\end{array}$ \\
\hline $\mathrm{f}$ & Ratio of the drag coefficient to Stokes drag \\
\hline $\operatorname{Re}_{\mathrm{p}}$ & Reynolds number of the particle \\
\hline $\mathrm{R}$ & Riser pipe radius \\
\hline $\mathrm{d}$ & Size of solids tracer particles \\
\hline $\mathrm{G}_{\mathrm{s}}$ or $\mathrm{M}_{\mathrm{s}}$ & Solids circulation rate \\
\hline$\alpha_{\mathrm{s}}$ & Solids fraction \\
\hline $\mathrm{m}$ & Solids or particle loading \\
\hline$\Phi$ & Spehericity \\
\hline $\mathrm{R}^{2}$ & Square of the correlation ratio \\
\hline $\mathrm{s}$ & Standard deviation \\
\hline St & Stokes number \\
\hline S & Struhal number \\
\hline $\mathrm{k}$ & Sublimation constant \\
\hline
\end{tabular}




\begin{tabular}{|c|c|}
\hline SS & Sum of squares \\
\hline $\mathrm{U}_{\mathrm{g}}$ & Superficial gas velocity \\
\hline $\mathrm{U}_{\mathrm{t}}$ & Terminal velocity \\
\hline $\mathrm{k}$ & The kinetic energy of the turbulence \\
\hline$\tau_{\mathrm{K}}$ & $\begin{array}{c}\text { The Kolmogorov time scale, which describes the smallest time scales of the } \\
\text { turbulence in the continuous phase }\end{array}$ \\
\hline $\mathrm{F}_{\alpha}$ or $\mathrm{F}^{*}$ & Theoretical F ratio \\
\hline $\mathrm{K}$ & Total number of categories or groups \\
\hline $\mathrm{N}$ & Total number of measurements \\
\hline $\mathrm{N}$ & Total number of measurements made \\
\hline$\dot{Q}_{c o_{2}}$ & Tracer gas flow rate \\
\hline $\mathrm{s}^{2}$ & Variance \\
\hline $\mathrm{V}_{\mathrm{S}}$ or $\mathrm{U}_{\mathrm{s}}$ or & Velocity of the solids \\
\hline $\mathrm{U}_{\mathrm{p}}$ or $\mathrm{V}_{\mathrm{p}}$ & Viscosity of the gas(air) \\
\hline$\mu_{\mathrm{g}}$ & Voidage \\
\hline$\varepsilon$ & Voidage at minimum fluidization \\
\hline$\varepsilon_{\mathrm{mf}}$ & \\
\hline
\end{tabular}




\section{Chapter I \\ Introduction and Literature Review}

\subsection{Background}

Circulating fluidized bed (CFB) can be defined as a fluidized bed with a steady recirculation of solids through a gas-solid separator (Kunii and Levenspiel, 1991). The solid circulation rate should be sufficiently high to ensure uniformity throughout the bed. There are many types of gas-solid reactors have been applied in many different processes. For example, they are used extensively in the petroleum industry and are also used to provide energy from the combustion of coal. CFB operates at higher gas velocities than conventional fluidized bed processes, giving CFB reactors many distinct advantages. Some of these include better contact efficiencies due to the slip between the two phases, more uniform distribution of solids due to reduced gas by-passing, reduced axial back-mixing and excellent heat and mass transfer rates (Grace et al., 1997).

There are two types of CFBs: the riser and the downer, these refer to where the main reaction of interest is occurring. In the riser, the gas-solid suspension travels up against the force of gravity and in the downer the suspension flows down with the force of gravity. Although risers possess advantages over the downer processes, they are hampered by the non-uniform flow structure. This condition reduces the contact efficiency between the two phases and leads to skewed residence time distributions. The backmixing leads to skewed residence time distributions of 
gas and solids within the reactor. This flow can be a problem when the reaction occurs in a very short contact time.

The emissions from a CFB combustor are closely linked with how the gas and the solids interact in the riser, i.e., the riser hydrodynamics. Despite the use of CFB's in fossil fuel combustion, the effects of solids and gas mixing in the dense entry region are still not well known. This situation is due to the fact that most of the studies in CFBs have been carried out for the fully developed regime and for low flux and low density conditions. There are only a few studies which have concentrated mostly on the gas-solids mixing in the dense entry region. However, the available information on gas mixing is even scarcer. It has been observed that the gas mixing in CFB combustors is generally poor and the incomplete mixing of air and fuel can be a major problem (Koksal, 2001). The information on the effects of the design and the mixing of the solids and gas becomes crucial for the combustor performance. A typical CFB configuration is shown in Figure 1.1 (Koksal, 2001). Quantitative understanding of the hydrodynamics in CFBs is essential for the scale-up and design of the processes taking place in a CFB. The yield in a gas conversion reaction, the combustion efficiency and emissions in fossil fuel combustion, the heat and mass transfer between gas and solids, the wall-to-bed heat transfer coefficients all depend on how the gas and solids are distributed and mixed in the riser.

The two main operational parameters in CFBs are superficial gas velocity $\mathrm{U}_{\mathrm{g}}$ and solids circulation rate (solids mass flux) $\mathrm{G}_{\mathrm{s}}$. The gas velocity is an independent 
operating variable defined as the volumetric flow rate of the total fluidization gas divided by the riser cross-sectional area. The circulation rate can be either independent or dependent based on the design of the solids inventory and is defined as the mass of the solids passing through a unit riser cross-sectional area per unit time (Berruti et.al., 1995).

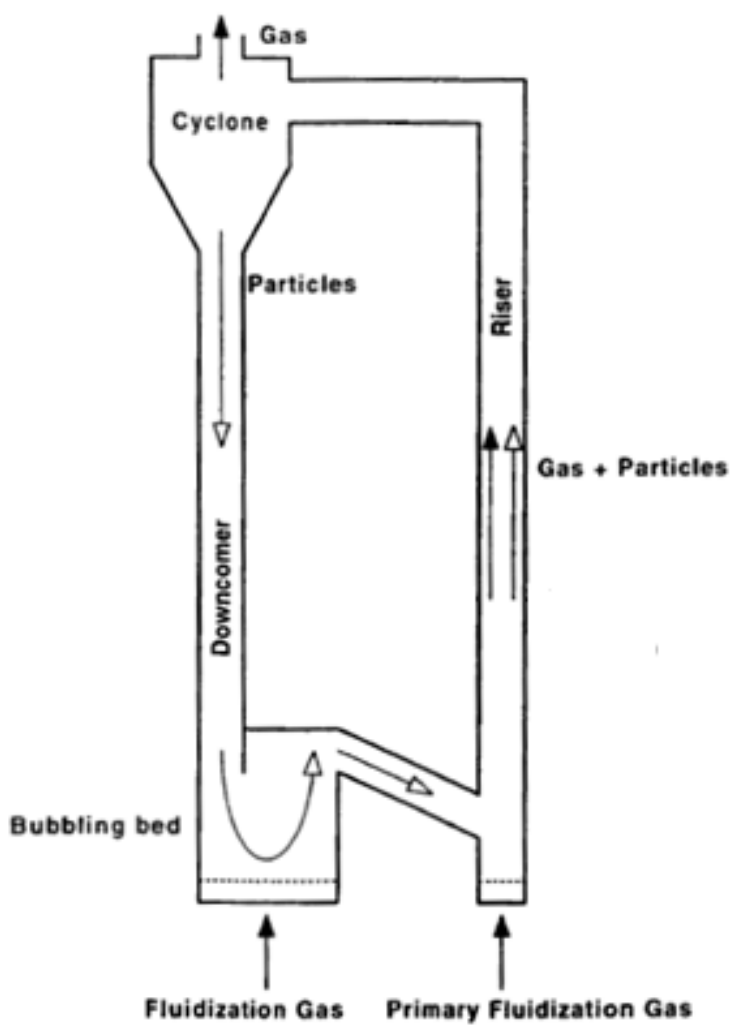

Fig1.1 Typical configuration of a CFB (Koksal, 2001)

Other design and operating parameters which affect the gas-solid flow pattern in a CFB are geometry of the riser (size, shape, inlet and exit configurations), riser wall roughness, particle properties, particle size distribution and secondary air injection (Arena et. al., 1992; Brereton and Grace, 1993; Kunii and Levenspiel, 1995; Zhou et al., 1996; Pugsley et. al., 1997; Arena, 1997). 
The gas-solid hydrodynamics inside the riser of a CFB is inherently complex. From purely fluid mechanical point of view, the problem can be seen as the flow of a turbulent gas-solid two-phase mixture in a vertical pipe or channel. The particles are neither small enough to follow the gas motion faithfully nor course enough to be unaffected by the gas. In addition to the effects of the gas the particle motion is equally affected by particle-particle and particle-wall collisions. The gas flow dynamics, in turn is also altered by the presence of the particles. The interactions between the gas and particles via viscous drag and between particles themselves via collisions produce complex solids congregation forms at different length scales such as clusters, streamers, swarms, and sheets (Lim et al., 1995).

These solids congregations form and disintegrate continuously. A duster has a slip velocity (relative velocity between the particles and the surrounding gas) of an order of magnitude larger than the terminal velocity of a single particle (Yerushalmi, 1986). This unique feature is the main cause of the improved heat and mass transfer between the particles and the gas in fast fluidization regime where clustering effects are dominant (Koksal, 2001).

\subsection{Mixing in the Dense Entry Region}

Many extensive studies have been conducted on CFB systems (Yemshalmi and Avidan, 1985; Reh, 1985; Grace, 1990, Contractor and Chaouki, 1991, Berruti, et al., 1995). Most of these studies have reported CFB data for systems operating at relatively low suspension densities (rarely above $2 \%$ solids by volume in the fully developed region), low gas velocities $(<10 \mathrm{~m} / \mathrm{s})$ and modest solids circulation rates 
$\left(<100 \mathrm{~kg} / \mathrm{m}^{2} \mathrm{~s}\right)$, conditions typical of CFB combustors. CFB risers consist of a dilute region towards the top (fully developed regime) and a relatively dense region near the bottom. The height of the dense region depends on the superficial gas velocity and the solids circulation rates. The fully developed regime has been subjected to a much wider investigation than the dense bed regime.

Very few systematic measurements of local parameters are available for high-flux dense systems. Studies conducted in the lower dense zone of CFB risers at relatively low circulation rates $\left(<100 \mathrm{~kg} / \mathrm{m}^{2} \mathrm{~s}\right)$ indicate differing observations. Some (Bolton and Davidson, 1988; Werther, 1994; Bai et al., 1995) indicate the lower dense region to be in the turbulent bed regime. While others (Ishii and Horio, 1991; Johnsson et al., 1992; Svensson et al., 1996) suggest the region to be a bubbling fluidized bed. In the present study we use amplitude analysis to conduct fluidization regime classification. A statistical measure for the amplitude of the pressure fluctuations is used to mark the transition between a bubbling bed and a turbulent bed as a result of a change in the superficial gas velocity. This concept was first proposed by Yerushalmi and Cankurt (1998). They defined this gas velocity for which the amplitude reaches a maximum as the transition velocity $U_{c}$. Depending on bed size, aspect ratio and solids used this velocity coincides with the velocity for which the bed is slugging at a maximum slug size or with the velocity for which the single bubble bed reaches maximum bubble size. Except for some recent work by Wei et al. (1997) in the bottom region of a CFB riser, no measurements of local solids flux under high density conditions are available 
in the literature. Although previous studies give some insight into the flow structure of the dense zone in CFB risers the solids circulation rates and gas velocity employed are well below those in commercial fluid catalytic cracking installations (see Table 1.1). Table 1.1 summarizes hydrodynamic studies with solids circulation rates in excess of $200 \mathrm{~kg} / \mathrm{m}^{2} \mathrm{~s}$. There is clearly a need for more fundamental research to study both global and local flow characteristics of CFB systems operated at high solids fluxes and suspension densities. The flow regimes and the associated suspension flow behavior must be considered or taken into account while modeling a CFB reactor. Improved understanding of the flow dynamics in high density circulating fluidized bed systems should enable better understanding of the advantages and limitations of HDCFB reactors. The present project was initiated to provide improved understanding of high density/high flux CFB systems, where the dense and dilute zones coexist in the riser as well as for conditions where the dense region occupies the whole column.

From the industrial application point of view this study is important because:

- Fluid Catalytic Cracking (FCC) is still by far the major industrial CFB system and improving the system may lead to substantial improvements.

- Better understanding of the fundamentals of high density/high flux systems could improve the design and increase the applicability and capacity of other CFB processes. 
- Increasing the suspension density even beyond that presently used in FCC reactors may lead to new applications requiring high solid/gas feed ratios and high solids hold-up (e.g. Shohji et al., 1983; Shaheen, 1983;

Stapinis, 199 1; Leuenberger and Wilbert, 1987; Park and Gau, 1986;

Martin et al., 1992).

- Developing the HDCFB should lead to high solids process capacity while maintaining good gas-solids contacting.

\begin{tabular}{|c|c|c|c|c|c|c|}
\hline Author & $\begin{array}{c}\text { Riser } \\
\text { (D× H) } \\
\text { mm } \times \mathbf{m}\end{array}$ & $\begin{array}{l}\text { Solids } \\
\text { feed } \\
\text { device }\end{array}$ & Particles & $\begin{array}{c}\mathbf{U}_{\max } \\
\mathrm{m} / \mathrm{s}\end{array}$ & $\begin{array}{l}\mathrm{G}_{\mathrm{s}, \mathrm{max}} \\
\mathrm{kg} / \mathrm{m}^{2} \mathrm{~s}\end{array}$ & $\begin{array}{c}\text { Measurement } \\
\text { technique }\end{array}$ \\
\hline $\begin{array}{l}\text { Yerushalmi et al. } \\
(1976,1978)\end{array}$ & $\begin{array}{l}76 \times 7.2 \\
152 \times 8.2\end{array}$ & $\begin{array}{l}\text { J-valve } \\
\text { J-valve }\end{array}$ & $\begin{array}{l}\text { FCC } \\
\text { FCC }\end{array}$ & $\begin{array}{l}7 \\
5\end{array}$ & $\begin{array}{l}213 \\
220\end{array}$ & $\begin{array}{l}\text { Differential } \\
\text { pressure }\end{array}$ \\
\hline $\begin{array}{l}\text { Arena et al. } \\
(1988,1991)\end{array}$ & $\begin{array}{l}41 \times 6.4 \\
400 \times 10.5\end{array}$ & $\begin{array}{l}\text { Slide valve } \\
\text { Loop seal }\end{array}$ & $\begin{array}{l}\text { Sand } \\
\text { Sand }\end{array}$ & $\begin{array}{l}7 \\
6\end{array}$ & $\begin{array}{l}600 \\
260\end{array}$ & $\begin{array}{l}\text { Differential } \\
\text { pressure }\end{array}$ \\
\hline $\begin{array}{l}\text { Galtier and } \\
\text { Pontier (1989) }\end{array}$ & $300 \times 22$ & \begin{tabular}{|l|}
$\begin{array}{l}\text { Mech. } \\
\text { valve }\end{array}$ \\
\end{tabular} & FCC & 20 & 400 & $\begin{array}{l}\text { Gamma-ray } \\
\text { absorption }\end{array}$ \\
\hline $\begin{array}{l}\text { Patience et al. } \\
\text { (1991) }\end{array}$ & $82.8 \times 5$ & L-valve & Sand & 8 & 240 & $\begin{array}{l}\text { Differential } \\
\text { pressure }\end{array}$ \\
\hline $\begin{array}{l}\text { Azzi et al. } \\
(1991)\end{array}$ & $\begin{array}{l}750,950 \\
\text { mm dia. }\end{array}$ & NA & FCC & 15 & 1000 & $\begin{array}{l}\text { Momentum probe } \\
\text { Gamma-ray } \\
\text { absorption }\end{array}$ \\
\hline $\begin{array}{l}\text { Contractor et al. } \\
\text { (1991) }\end{array}$ & $150 \times 27.4$ & NA & FCC & 4.4 & 230 & $\begin{array}{l}\text { Differential } \\
\text { pressure } \\
\text { Radioactive tracer }\end{array}$ \\
\hline $\begin{array}{l}\text { Mori et al. } \\
\text { (1991) }\end{array}$ & $50 \times 4.55$ & & FCC & 7 & 400 & $\begin{array}{l}\text { Differential } \\
\text { pressure }\end{array}$ \\
\hline $\begin{array}{l}\text { Dry and } \\
\text { Christensen } \\
\text { (1989) }\end{array}$ & $90 \times 7.2$ & NA & FCC & 8 & 200 & $\begin{array}{l}\text { Differential } \\
\text { pressure }\end{array}$ \\
\hline $\begin{array}{l}\text { Martin et al. } \\
\text { (1992) }\end{array}$ & $\begin{array}{l}190 \times 11.7 \\
940 \times 27\end{array}$ & Slide valve & $\begin{array}{l}\text { FCC } \\
\text { FCC }\end{array}$ & 6 & 216 & $\begin{array}{l}\text { Gamma ray } \\
\text { absorption } \\
\text { Radioactive tracer }\end{array}$ \\
\hline $\begin{array}{l}\text { Donsi and } \\
\text { Osseo (1994) }\end{array}$ & $57^{*} \times 4.6$ & \begin{tabular}{|l} 
Butterfly \\
valve
\end{tabular} & Glass & 30 & 500 & $\begin{array}{l}\text { Differential press. } \\
\text { Laser Doppler } \\
\text { anemometry }\end{array}$ \\
\hline $\begin{array}{l}\text { Pugsley et al. } \\
\text { (1993) }\end{array}$ & $50 \times 5.0$ & & Sand & 7 & 400 & $\begin{array}{l}\text { Differential } \\
\text { pressure }\end{array}$ \\
\hline $\begin{array}{l}\text { Wei et al. } \\
(1994)\end{array}$ & $186 \times 8$ & Slide valve & $\overline{\text { FCC }}$ & 8.5 & 215 & $\begin{array}{l}\text { Differential press. } \\
\text { Optical fiber imaging }\end{array}$ \\
\hline $\begin{array}{l}\text { Karri and } \\
\text { Knowiton } \\
\text { (1996) }\end{array}$ & $300 \times 13$ & L-valve & Catalyst & 8.1 & 586 & $\begin{array}{l}\text { Differential } \\
\text { pressure }\end{array}$ \\
\hline
\end{tabular}

- hydraulic diameter of a column of $40 \mathrm{~mm} \times 100 \mathrm{~mm}$ cross-section

Table1.1 Contemporary existing research conducted 


\subsection{Objectives of this study}

This thesis has three basic goals. The first goal of this study is to investigate the dispersion of a stream of solids being injected into a riser. This part deals with effects of the operating conditions on the mixing of the solids and gas in the dense entry region of the riser. The second part deals with developing design basis equations for the helping in designing combustors having a good mixing process of solids and gas.

The specific goals of this work are;

- To develop a tracer gas technique to study and characterize the flow and distribution of the solids being fed asymmetrically or symmetrically into a circulating fluidized bed riser, at the lower dense entry regions.

- To enhance our understanding and characterize the gas mixing in the dense entry region of a circulating fluidized bed riser.

- To develop a Statistical Design Basis Equations to assist engineers in designing more efficient gasifier reactors.

\subsection{Significance of the Study}

This study was conducted at the National Energy Technology Laboratory on their cold flow test facility mimics commercial scale transport reactors with side entry of solids into the vertical riser. Figure 1.2 illustrates how this study differs from most of the existing work done on gas-solids mixing. Figure 1.2 represents a classification for gas-solid suspensions proposed by Elgobashi. The figure shows that, when the suspension is very dilute $\left(\alpha_{s}<10^{-6}\right)$ the particles have no effect on the 
turbulent motion of the continuous phase, but their motion can be governed by the turbulent motion of the continuous phase if their inertia is sufficiently small. This process is called "one way coupling". Here the fluid carrier influences the particles via drag and turbulence, but the particles have no influence on the fluid carrier. When the particle volume fraction is increased (say up to $a_{s}=10^{-3}$ ), the effects of the presence of the particles on the turbulent motion of the continuous phase can be observed. This process is called "two way coupling". Here the fluid carrier influences the particulate phase via drag and turbulence, but the particles in turn influence the carrier fluid via reduction in mean momentum and turbulence. When the particle volume fraction exceeds a certain value (say $\alpha_{s}>10^{-3}$ ) the relative distance between particles is small enough so that particles collide. This process is called "four-way coupling. Here there is two-way coupling plus particle pressure and viscous stresses due to particles (four-way coupling). In most fluidization applications, four-way coupling is expected to occur, at least in the dense regions of the bed.

Most of the existing studies on the hydrodynamics of CFB's are carried out by feeding the fluidizing gas and solids coaxially from the bottom of the riser (Arena, 1997). Not many studies deal with the system hydrodynamics, i.e., the axial pressure drop, suspension density and particle velocity profiles for an industrial scale asymmetrically loaded riser. This study is unique as it deals with high solids density or fraction conditions indicated by the shaded region. In this region, there is a coupling between the solids on the air in the dense entry region of the riser. This 
coupling leads to an important phenomenon called turbulence phenomenon which will be discussed in detail in chapter V. The contemporary works on the hydrodynamics of the riser focus on the low solids density or solids fraction conditions and on the fully developed region of the riser. In such cases there is only a one way coupling.

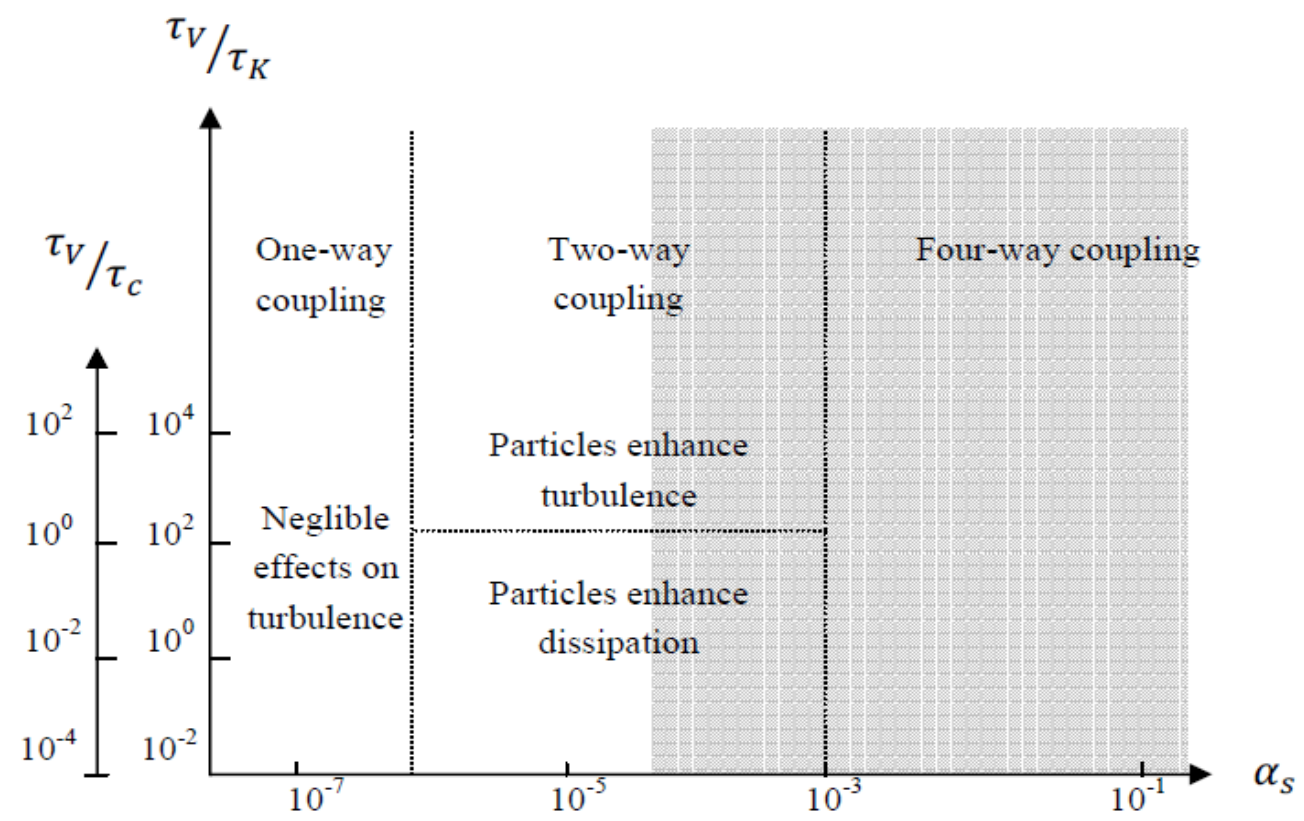

Fig.1.2. Elgobashi's (1991) classification of particle turbulence coupling according to Peirano and Leckner (1998)

\subsection{Structure of the thesis}

A general review of circulating fluidized beds is presented in Chapter I while leaving detailed discussion for presentation where appropriate in later chapters. Chapter II deals with (a) the experimental set up and instrumentation used for this research, (b) the operational procedure for this research and (c) test matrix implemented for this research. Chapter III covers the literature review of the solids and gas mixing process for a high flux, high density CFB riser with an emphasis on 
the dense entry region. Chapter IV deals with the analysis of the tracer gas dispersion results. Chapter $\mathrm{V}$ focuses on the gas solids interaction and flow behavior in the dense entry region of the riser. Chapter VI deals with the statistical formulation of the design basis equation based on the dispersion data. Chapter VII deals with the overall conclusions and future recommendations. 


\section{Chapter II}

\section{Experimental Set-Up, Instrumentation and Procedure}

\subsection{Achieving high density/high flux conditions in risers}

The suspension density in a circulating fluidized bed riser can be raised either by increasing the solids flux at a fixed superficial gas velocity or by lowering the superficial gas velocity while holding the solids flow rate constant. To maintain steady operation in the fast fluidization regime there are, however limits on the circulation rate for a given gas velocity and on the velocity for a fixed solids circulation flux. The limitations are imposed by either of two modes of operational instabilities which may take place in the riser. These problems are discussed next together with methods of avoiding them.

\subsection{Classical choking}

Gradually increasing the solids circulation rate in a CFB riser operating at a fixed superficial gas velocity eventually leads to a point at which the up flowing gas can no longer support the particle suspension causing it to collapse. On the other hand, when the superficial gas velocity is reduced at a fixed solids flux a similar phenomenon may also occur. This operational instability is generally referred to as choking (Zenz and Othmer, 1960) or Type C (Classical) choking (Bi et al.. 1993). It becomes impossible to operate the riser in the fast fluidization mode at a superficial gas velocity lower than that corresponding to choking at a fixed solids flux. To operate at a higher solids flux than that at the choking point for a given superficial 
gas velocity leads to severe slugging for slugging systems, or the bed switches directly into the bubbling regime for non-slugging systems. The onset of classical choking is dependent on gas and solids properties as well as on the size and geometry of the column. If suitable choices of these variables are made e.g. small particles and a riser of sufficiently large diameter the unit can be operated in the fast fluidization flow regime over a wide range of solid circulation rates. Choking investigations have led to a number of correlations to determine the onset of the instability. An equation proposed by Yousfi and Gau (1974)

$$
\frac{U_{c h}}{\sqrt{g d_{p}}}=32 R e_{t}^{-0.06}\left(\frac{G_{s}}{\rho_{g} U_{c h}}\right)^{0.28}
$$

has been found to be most accurate in predicting the classical choking velocity in Group A particles (Teo and Leung, 1984; Bi et.al., 1993).

\subsubsection{Equipment-induced instabilities}

Two other forms of instability may also occur in a CFB system while attempting to increase the riser solids hold-up before the classical transition point is reached. The first occurs if the gas blower is unable to provide a sufficient pressure head to overcome the pressure drop in the riser and maintain the particles in suspension (Zenz and Othmer. 1960; Doig and Roper, 1963; Leung et ai., 1971). The second results from pressure imbalance between the riser and the solids return leg making steady state operation impossible (Knowlton and Bachovchin, 1976; Takeuchi et al., 1986; Bader et al., 1988; Hirama et al., 1992).

The down-comer-riser loop pressure balance has been a subject of several theoretical analyses (Weinstein et al.. 1983; Kwauk et al.. 1986; Arena et al., 1987; 
Rhodes and Geldart, 1987; Rhodes and Laussmann, 1992; Yang, 1988; Breault and Mathur, 1989; Horio and Takei. 1991; Bi and Zhu, 1993). These researchers have provided useful information on improving $\mathrm{CFB}$ design and operation. Bi and Zhu (1993) proposed that high suspension densities and high solids fluxes could be achieved by a combination of factors. These factors are high solids inventories, large down-comer-to-riser diameter ratio, a low pressure drop solid feeder and minimizing pressure drops in solids separation devices and fittings doing the loop, a proper blower and suitable particle size and riser diameter combinations.

Other methods, which do not rely on the down-comer pressure head, have been used previously for feeding solids to high-density risers. Yousfi and Gau (1974) superimposed pressure on a fluidized bed feeder installed in the solids return leg and adjusted the pressure in the feeder with an exhaust valve. Screw feeders have also been used (Drahos et al.. 1988; Mon et al.. 1991 ; Hirama et al.. 1992). While these alternatives may overcome the disadvantages of gravity feeding, they are cumbersome from an industrial point of view and may not be able to supply the high solids rates required in commercial units.

The schematic of the single-loop cold flow circulating fluidized bed used for this work is shown in the figure 2.1. This unit is located at the National Energy Technology Laboratory, at Morgantown, West Virginia. As a result, this work was constrained by the locations of the ports so that we had to use the available ports for data acquisition and follow the existing test matrix. 


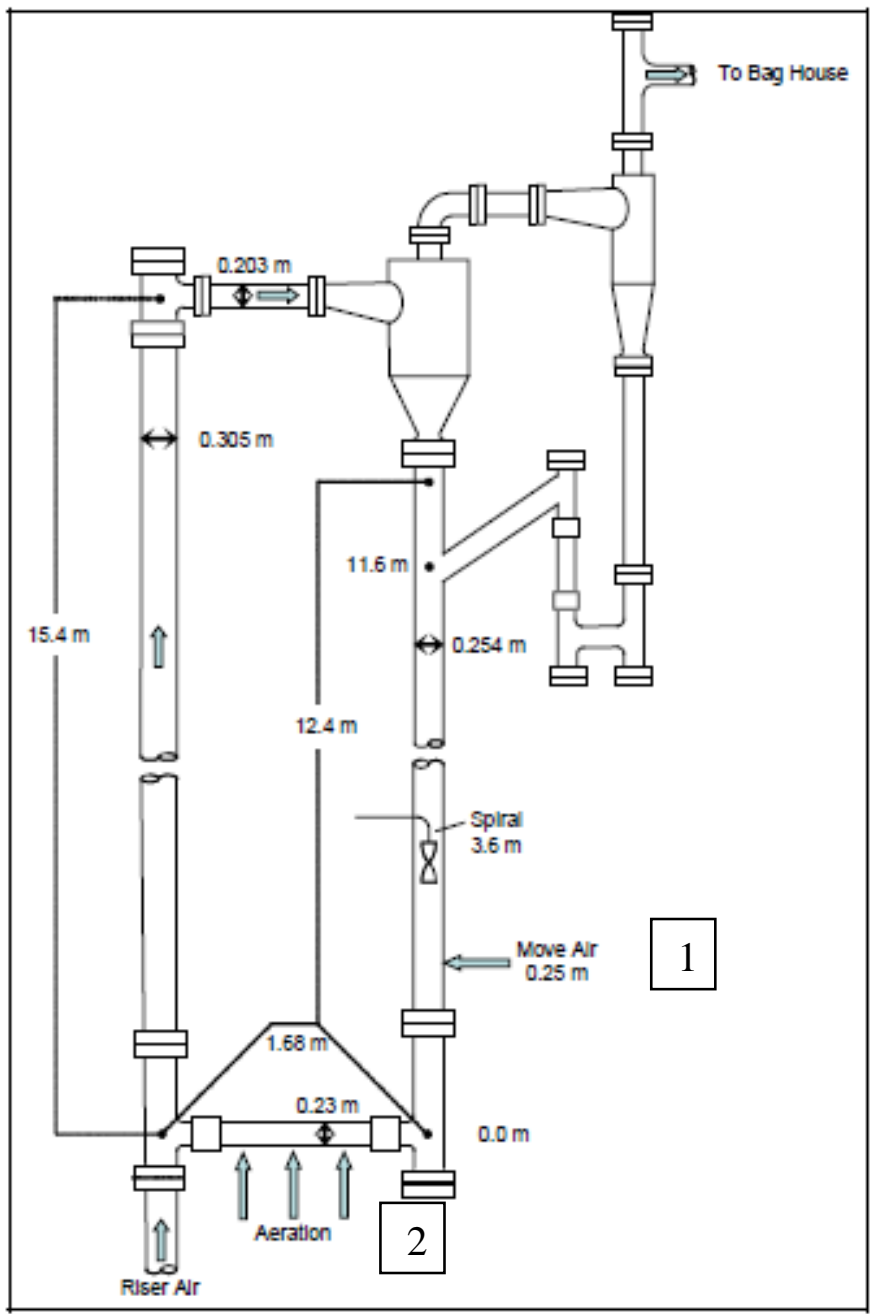

Figure 2.1 Schematic Diagram of the NETL 0.3m diameter, 15m high circulating fluidized bed cold model used in this study

\subsubsection{Experimental Apparatus}

Some of the main considerations that goes into the design of a CFB system are design were:

1. The unit is capable of operating at superficial gas velocities and solids fluxes in the riser which are interest to this study i.e., which are representative of the conditions of in FCC reactors and other high density reactors as discussed 
in chapter 1 . The air velocity in the riser should be between 3 and $12 \mathrm{~m} / \mathrm{s}$ and the solids flux should exceed $200 \mathrm{~kg} / \mathrm{m}^{2} \mathrm{~s}$ extending to at least $500 \mathrm{~kg} / \mathrm{m}^{2} \mathrm{~s}$.

2. The riser is tall enough that a substantial portion of it operates beyond the acceleration region. It should have as large a diameter as possible to make the results credible and useful for scale-up.

3. The unit is modular and flexible in construction to allow insertion or exchange of column sections permitting variations in inlet and exit configurations, height and even riser column diameter.

4. The unit is transparent to allow visual observation of the flow process/phenomenon.

The cold flow facility at NETL satisfies all the four requirement mentioned above. The riser was constructed of flanged steel and booted acrylic sections. The solids entered the riser from a $0.23-\mathrm{m}$ (ID) side port located $0.27-\mathrm{m}$ above the gas distributor. The solids entry side port was connected to a non-mechanical valve; an. L-Valve. Solids exited the riser through a $0.20-\mathrm{m}$ port perpendicular to the riser at a point 15.45 - $\mathrm{m}$ above the solids entry location (centerline to centerline). The tee at the top of the riser extended about $1.2-\mathrm{m}$ beyond the exit port. The riser velocities were corrected for temperature and pressure as measured at the base of the riser. Twenty incremental differential pressures were measured along the length of the riser using transmitters calibrated within $0.1 \%$ of full-scale or about $2 \mathrm{~Pa} / \mathrm{m}$. The other primary response measurement was the overall riser pressure drop and it was calibrated within $0.45 \mathrm{~Pa} / \mathrm{m}$. Mass circulation rates were continuously recorded by 
measuring the rotational speed of a twisted spiral vane located in the packed bed region of the standpipe [4]. As the solids flow down the standpipe, they rotate the spiral vane. The rotational rate of the spiral, which is a function of the rate of the down flowing solids, gives the value of the solids circulation rate.

This rate of the spiral rotation was converted to a mass flux using the measured packed-bed density shown in Table 2.1, and assuming a constant void fraction at the point of measurement. A microscopic image of the beads is shown in figure 2.2 .

\begin{tabular}{|c|c|c|}
\hline Quantity & Units & Value \\
\hline Density of Solids $\left(\rho_{\mathrm{s}}\right)$ & $\mathrm{kg} / \mathrm{m}^{3}$ & 2426 \\
\hline$\rho_{\mathrm{b}}$ & $\mathrm{kg} / \mathrm{m}^{3}$ & 1384 \\
\hline $\mathrm{d}_{\mathrm{sv}}$ & $\mu \mathrm{m}$ & 62 \\
\hline $\mathrm{U}_{\mathrm{t}}$ & $\mathrm{m} / \mathrm{s}$ & 0.225 \\
\hline $\mathrm{U}_{\mathrm{mf}}$ & $\mathrm{m} / \mathrm{s}$ & 0.085 \\
\hline $\mathrm{C}_{\mathrm{mf}}$ & & 0.421 \\
\hline$\varphi$ & & 0.86 \\
\hline
\end{tabular}

Table 2.1 high density PPE bead characteristics

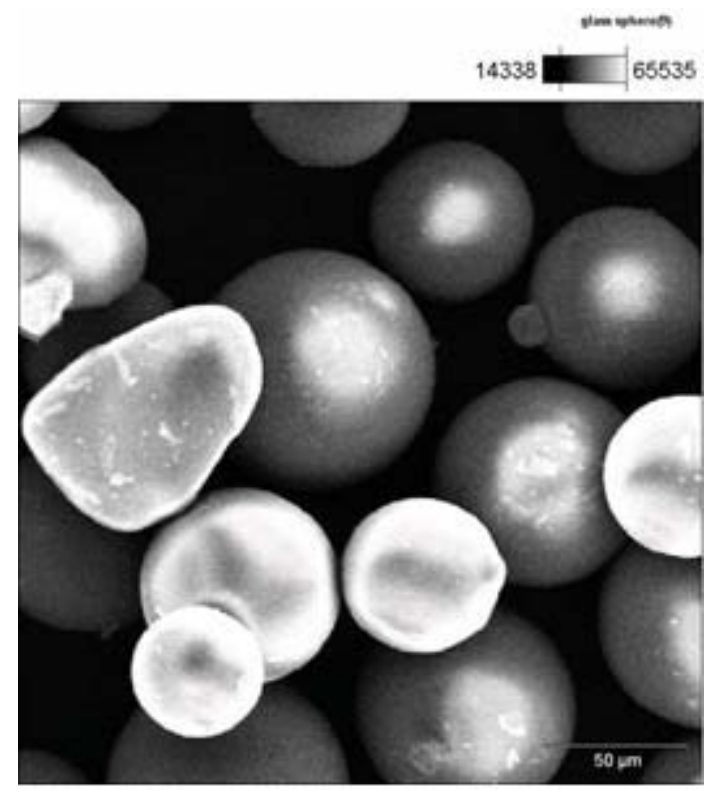


Fig2.2 HDPE beads SEM micrograph

The solids circulation rate was varied by controlling the aeration the aeration at the base of the standpipe and by adjusting the total system inventory to increase the standpipe height. Steady state conditions were defined as holding a constant set of flow conditions and maintaining a constant response in the pressure differentials over a 5-min period. All steady state test results represent an average over that 5min period. During an experiment, the air velocity in the riser was controlled at a constant level. The superficial riser velocity was the summation of the flow at the base of the riser with that at the base of the lift-leg in the loopseal. Steam was introduced into the air supply header as needed to maintain the relative humidity at approximately $40 \%$. During an experiment, the air velocity in the riser was held constant.

This calibrated volumetric measurement was converted to a mass flux using the measured void fraction of 0.42 in the packed bed and assuming that the void fraction at the point of measurement was constant. Analysis of the standpipe pressure profile, estimated relative gas-solids velocities, and bed heights have indicated that this constant voidage estimate was reasonable over the range of operating conditions reported here. Measurements of the capacitance across the standpipe at the same location as the spiral indicated that some increase in bed density could be observed at the highest solids fluxes; however, little or no deviation was observed in the linear relationship between riser pressure drop and measured circulation rate for each superficial gas velocity. 


\subsection{Instrumentation}

The cold flow high flux high density circulating fluidized bed project is operated and controlled by a combination of hardware and software components. The main control is implemented by Moore 352 single loop PID controllers in the control room. This gives the operator direct access to control the most of the system from the control room. The data acquisition system used for recording all data obtained from the CFB unit was a Paragon data acquisition system.

\subsubsection{Air Flow Rate Measurements}

The primary air flow rates were measured by flow meters which use solenoid controlled orifice plates to regulate air flow. The differential pressure from each flow meter was measured by a differential pressure transducer having a range of 0-5 inches water column (Omega PX164 - 005D5V). The average air velocity in each of the corresponding pipes was found from the correlation suggested by the manufacturer as:

$$
V=1096.2 \sqrt{\frac{\Delta P}{\rho_{\text {air }}}}
$$

Where $\mathrm{V}$ is the velocity in $\mathrm{ft} / \mathrm{sec}, \Delta P$ is the differential pressure in inches of water and $\rho_{\text {air }}$ is the air density in $\mathrm{lb} / \mathrm{ft}^{3}$. The volumetric air flow rates were then obtained and recorded by multiplying the average velocity with the pipe cross-sectional area.

\subsubsection{Differential Pressure Measurements}

The riser air differential pressure measurements are obtained from standard industrial instruments (Rosemont PDT's). They are connected to various ports on 
the riser and produced an output of 4-20 milliamps signal representing the differential pressure. The 4-20 milliamps output signal is converted it to $1-5 \mathrm{~V}$ signal using a $250 \Omega$ resistor. This voltage signal is then sent as an input to an OPTO-22 0-5V input/output data acquisition module to be read by the Paragon data acquisition system.

\subsubsection{Solids Circulation Rate Measurements}

The solids circulation rate is measured by a spiral vane placed in the standpipe at 13 feet elevation. The spiral vane is shown in figure 2.3. As the bed material passes the spiral, it causes the spiral to rotate. There are several assumptions made in this process. These assumptions are:

- The data acquisition software averages encoder counts over 2 seconds.

- Assuming 1 foot of bed travel $=180$ degrees of spiral rotation $=64$ counts (ideally)

o Counts/Second gives bed velocity

- Assuming plug flow for bed flow

- Assuming cross sectional area of bed = area of flow

- Bed Velocity $\times$ Area of Flow $=$ Bed Volumetric Flow

- Assuming the bulk density of the bed is known

$$
\text { o Bed Volumetric Flow } \times \text { Bulk Density }=\text { Bed Mass Flow }
$$

The spiral turns a shaft that is connected to an encoder. The encoder generates two pulse signals that are input to a digital input module (National Instruments) contained in an FPGA rack system. Labview software is used to 
determine the speed (rev/sec) of the spiral using a quadrature decoding of these signals. Labview then transmits this value to Paragon using an OPC interface between the two programs. Paragon uses the following equation to calculate the circulation rate given the rev/sec of the spiral from the encoder:

$$
\text { Solids Circulation Rate }=\text { Spiral } \frac{\mathrm{rev}}{\mathrm{S}} \times \text { Bed Density } l b / f t^{3} \times 57.4219 \times 0.5454 \times 128
$$

The Spiral (revolutions/s) is measured by the quadrature encoder. Bed density is entered by the operator. In house research (The Model Validation Group) at NETL came up with the other constants in the equation.

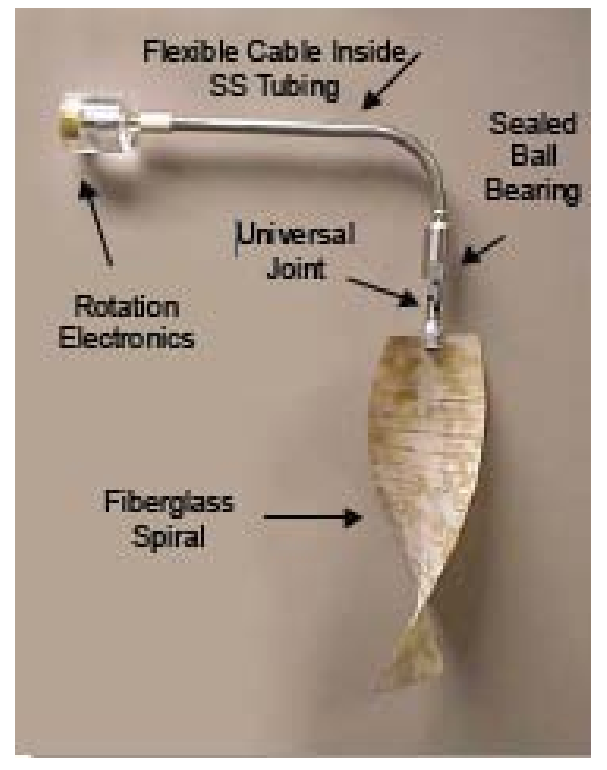

Fig 2.3 the spiral vane used for measuring solids circulation rate

\subsubsection{The Test Matrix}

\subsubsection{Initial work to determine the Test Matrix}

Several tests were conducted with the polyethylene beads in order to characterize the detailed hydrodynamic flow structure in the cold flow CFB riser. 
These tests were based on statistical factorial designs evaluating two primary operating parameters, the gas velocity taken at the exit of the riser and the mass circulation rate of solids being recycled into the riser.

\begin{tabular}{|c|c|c|}
\hline Material & Expression & Model Accuracy $\left(\mathrm{R}^{2}\right)$ \\
\hline PPE & $L g\left(G_{S}{ }^{*}\right)=\left(35.11-102.55 /\left(U_{g} / U_{t}\right)+80.85\left(U_{g} / U_{t}\right)^{2}\right.$ & 0.957 \\
\hline
\end{tabular}

Table 2.2 Equation to predict choking (Monazam et al 1999)

The test matrix included a center point operating condition which was duplicated each time the test matrix was conducted. This ensured that in the event that detailed local measurements taken on a given day could not be repeated, the uncertainty could be estimated from the set of duplicates taken at the center point operating condition. Each of the primary variables was tested at 3 levels. The experimental conditions were chosen based upon the relative distance from classical choking boundary. This boundary is depicted as the dashed teal line in figure 2.4 and is governed equation 1 in table 2.2. This equation was developed at NETL after an extensive analysis of different materials.

- In this analysis, 7 different granular materials were evaluated using the rapid transient analysis method pioneered by Perales et.al. (1990) and further developed by Monazam et al (2000) (Monazam et al. 2001).

- The 200 micron glass beads and the 750 micron polyethylene beads exhibited much lower choking velocities than could be reasonably explained by the saturated carrying capacity $\left(\mathrm{G}_{\mathrm{s}}{ }^{*}\right)$ equation expressed in table 2.2 . 
At the end of the analysis for the different materials and their relative transition velocities it became clear that some of the larger and denser materials behaved distinctly different from the others. For this reason a separate expression was developed and is used as the reference point for the test matrix. This expression shown in table 2.2 represents the observed transition from choking to turbulent to fast fluidized flow for the polyethylene beads.

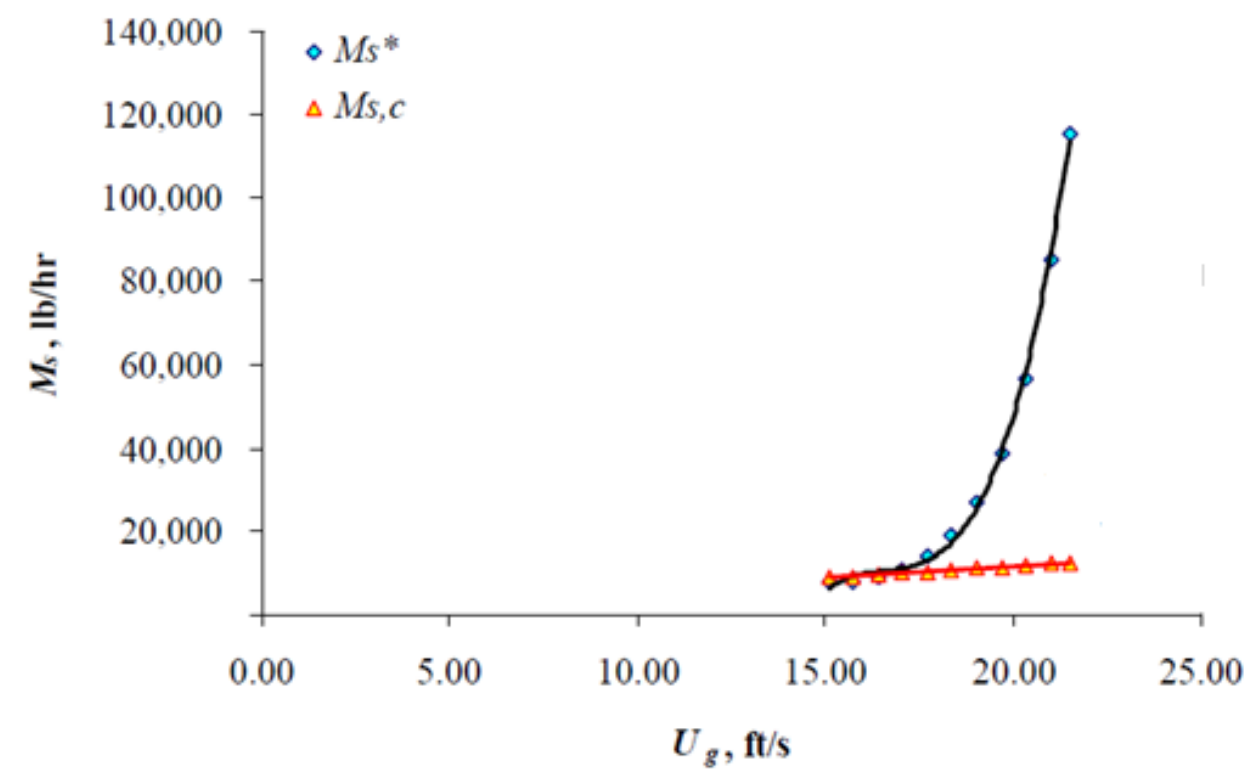

Fig2.4. Operating Regime for the $750 \mu \mathrm{m}$ Polyethylene beads representing the saturated carrying capacity and the choking velocities as taken from Yang's model and applied to the NETL CFCFB (Yang 1975).

The desired test matrix was chosen to span the space above the velocity in which the gas would reach its saturated carrying capacity (SCC). The test matrix is presented in figure 2.5 and is located below the SCC curve. The test conditions are represented by symbols on the $M_{s}-U_{g}$ plot and the operating conditions used during 
the test matrix are highlighted by the blue diamonds. This design represents a set of factorial points with a center point which have been transposed parallel to the SCC line. A dashed box displays these factorial points and the centroid of the test points/test matrix.

\begin{tabular}{|c|c|c|c|c|c|}
\hline Symbol & Ms $(\mathrm{kg} / \mathrm{s})$ & $\mathrm{Ug}(\mathrm{m} / \mathrm{s})$ & Gs $(\mathrm{kg} / \mathrm{sq} \cdot \mathrm{m} \mathrm{s})$ & $\begin{array}{c}\text { Mair(Dist) } \\
(\mathrm{kg} / \mathrm{s})\end{array}$ & $\begin{array}{c}\text { Mair(L-Valve) } \\
(\mathrm{kg} / \mathrm{s})\end{array}$ \\
\hline $\mathrm{a}$ & 1.386 & 5.486 & 27.353 & $4.87 \mathrm{E}-04$ & $4.10 \mathrm{E}-04$ \\
\hline $\mathrm{d}$ & 1.386 & 7.62 & 27.353 & $6.46 \mathrm{E}-04$ & $4.01 \mathrm{E}-04$ \\
\hline $\mathrm{g}$ & 2.835 & 6.401 & 55.949 & $5.57 \mathrm{E}-04$ & $7.27 \mathrm{E}-04$ \\
\hline $\mathrm{e}$ & 2.835 & 7.62 & 55.949 & $6.77 \mathrm{E}-04$ & $7.27 \mathrm{E}-04$ \\
\hline $\mathrm{b}$ & 5.544 & 6.401 & 109.412 & $6.23 \mathrm{E}-04$ & $7.27 \mathrm{E}-04$ \\
\hline b & 5.544 & 6.401 & 109.412 & $6.22 \mathrm{E}-04$ & $4.10 \mathrm{E}-04$ \\
\hline f & 9.765 & 7.62 & 192.715 & $6.48 \mathrm{E}-04$ & $4.10 \mathrm{E}-04$ \\
\hline c & 11.34 & 7.62 & 223.798 & $7.43 \mathrm{E}-04$ & $7.27 \mathrm{E}-04$ \\
\hline
\end{tabular}

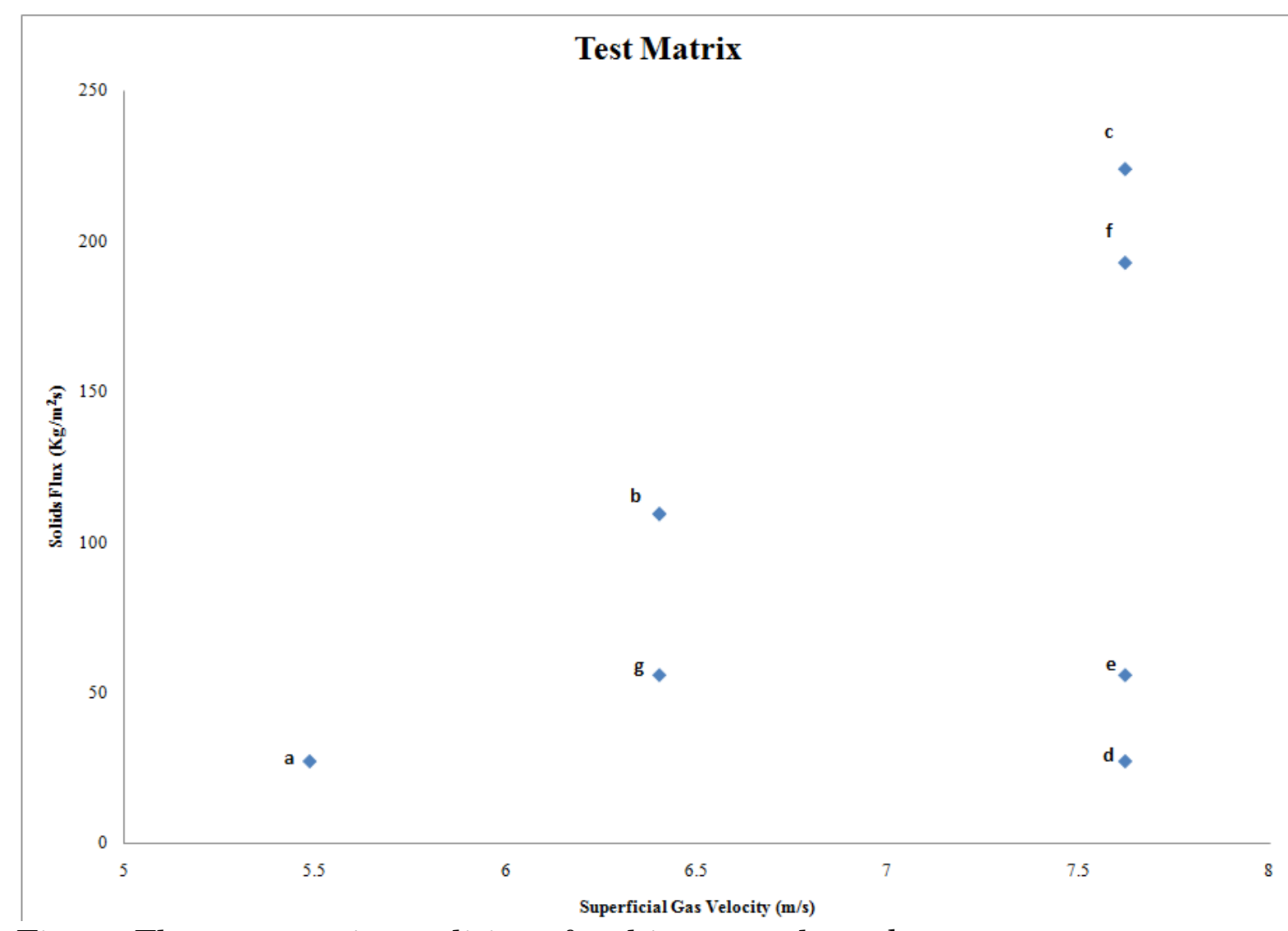

Fig2.5. The test matrix conditions for this research work 
There is an additional test point that was taken as part of the tests which represents a leaner riser operating condition as that used as the base condition. The base condition was conducted at $7.62 \mathrm{~m} / \mathrm{s}(25 \mathrm{ft} / \mathrm{s})$ and $4.105^{*} 10^{4} \mathrm{~kg} / \mathrm{hr}(90,000 \mathrm{lb} / \mathrm{hr})$, while the leaner condition was taken at the same gas velocity but with only and $1.021^{*} 10^{4} \mathrm{~kg} / \mathrm{hr}(22,500 \mathrm{lb} / \mathrm{hr})$ of solids being recirculated. In this way there are several points of identical gas velocities but having a range of solids circulation rates. The other set of conditions that exhibit this same characteristic are the points taken at $6.553 \mathrm{~m} / \mathrm{s}(21.5 \mathrm{ft} / \mathrm{s})$ at and $5.103^{*} 10^{3} \mathrm{~kg} / \mathrm{hr}(12,500 \mathrm{lb} / \mathrm{hr}) 2.012^{*} 10^{4} \mathrm{~kg} / \mathrm{hr}$ $(43,800 \mathrm{lb} / \mathrm{hr})$ and $3.504^{*} 10^{4} \mathrm{~kg} / \mathrm{hr}(77,500 \mathrm{lb} / \mathrm{hr})$ and at $5.486 \mathrm{~m} / \mathrm{s}(18 \mathrm{ft} / \mathrm{s})$ at $5.103^{*} 10^{3} \mathrm{~kg} / \mathrm{hr}(12,500 \mathrm{lb} / \mathrm{hr})$.

\subsection{Region Classification of the NETL Cold Flow CFB Riser}

This thesis is focused at the bottom dense region of the cold flow circulating fluidized bed riser. A detailed sketch of the NETL unit depicting the different regions is shown in figure 2.6. As mentioned in chapter 1 this technique is used as means to determine distribution of a stream of solids being injected into the riser. 


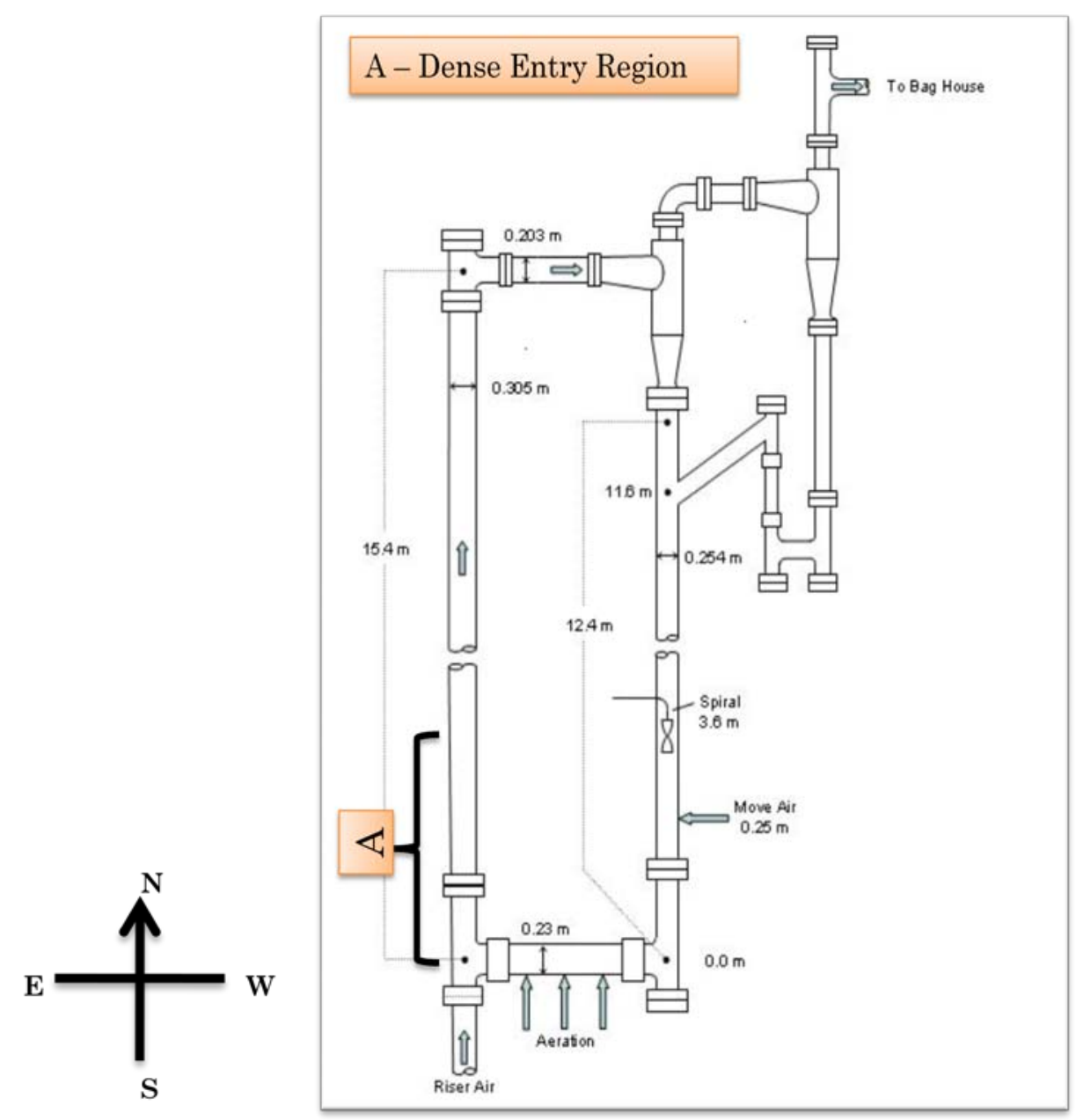

Fig2.6 Cold Flow CFB at NETL

The distribution measurements are limited to the lower levels of the dense entry region before the co-flowing stream disintegrated. The technique is also being used to determine the gas mixing in the dense entry region of the riser. The dense entry region of the riser is indicated by section (A) in figure 2.6. In the unit at NETL, the dense entry region ranges about $4.115 \mathrm{~m}$ (13ft) from the center line of the L-Valve (which is selected as ground $0(0 \mathrm{~m}$ or $0 \mathrm{ft})$. The stream of solids is fed into the riser base through the L-Valve, which is a non mechanical valve.

The injection of the $\mathrm{CO}_{2}$ tracer gas is made in the $\mathrm{L}$-Valve. The injection probe is so located that the probe is in the 6.35E-3 $\mathrm{m}$ from the base of the L-Valve. This ensured that the tracer gas was injected at center of the solids stream for all conditions. Injecting the tracer gas 
this way it is assumed that the solids moving through the $\mathrm{L}$-Valve, contains the $\mathrm{CO}_{2}$ tracer gas as it flows into the riser.

\subsection{The Dense Entry Region of the Riser}

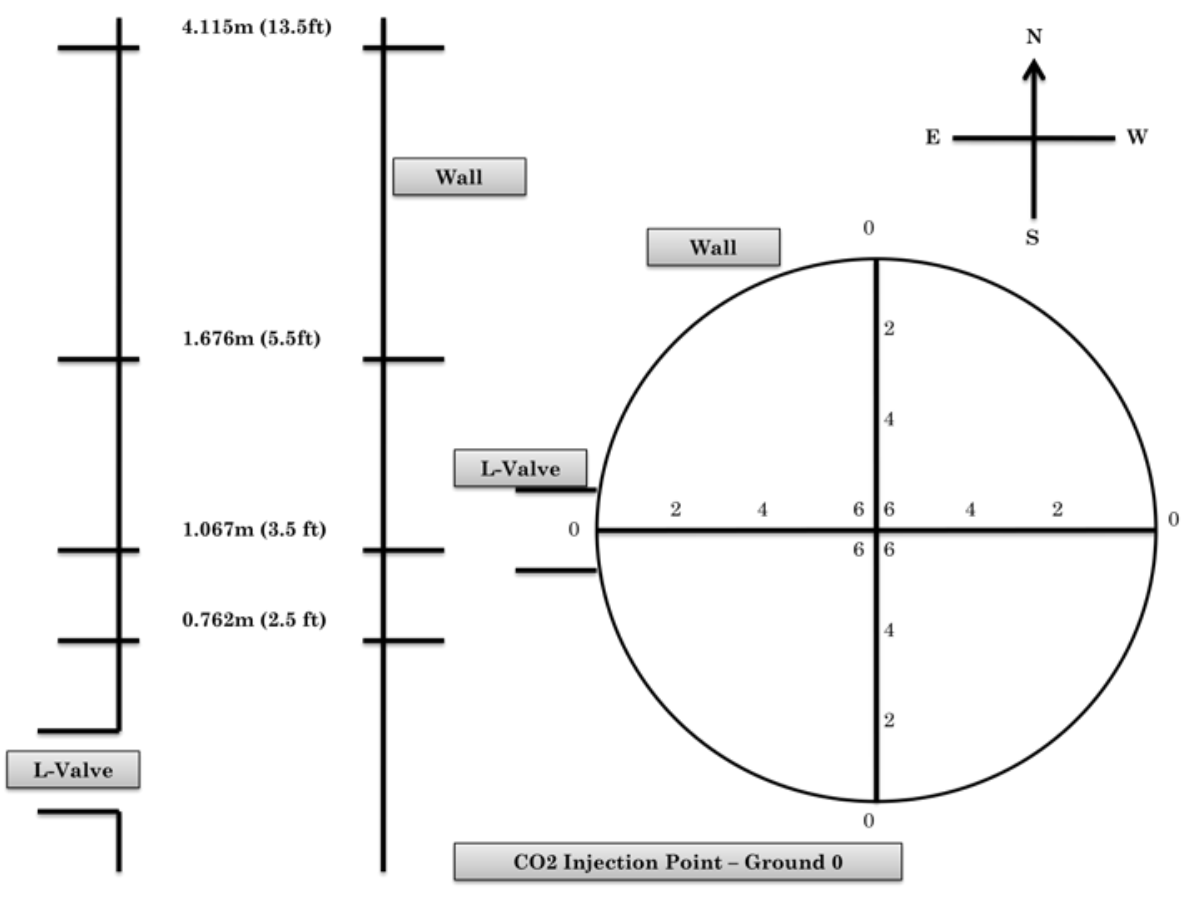

Fig2.7 Locations at which tracer gas measurements were taken in the dense entry region

Figure 2.7 shows the dense entry region of the riser as well as the elevations and radial locations at which measurements were taken. The center line of the L-Valve is the baseline and its elevation is $0 \mathrm{~m}$. As indicated in figure 2.7, the axial positions at which tracer has measurements were taken were $0.762 \mathrm{~m}, 1.067 \mathrm{~m}, 1.676 \mathrm{~m}$ and $4.115 \mathrm{~m}$. Two factors led to the selection of these axial elevations, one the primary focus was on the dense entry region of the riser and secondly, on the availability of the ports for measurement in the riser. The radial positions at which the measurements were made are also indicated in figure 2.8. For each axial position, 7 radial measurements were made, each radial position $0.051 \mathrm{~m}$ from each other. At each axial elevation measurements were made in the East-West and North-South directions. 
Repeats were measured at the center point of the riser for confidence interval calculations. The confidence interval determined at the center of the riser was assumed to be true for all the other radial positions.

\subsection{Probe Instrumentation}

\subsubsection{Tracer Gas Flow Rate}

The amount of the tracer gas injected is an important parameter that was set prior to the experiments. The linearity and the detectability of the measurements were also considered.

The atmospheric air contains $\mathrm{CO}_{2}$ at an average concentration of $0.05 \%$ by volume. Thus, the volumetric flow rate of $\mathrm{CO}_{2}$ tracer should be adjusted such that it gives a mean mixed concentration that is well above the normal $\mathrm{CO}_{2}$ composition of air. The $\mathrm{CO}_{2}$ in the atmospheric air is taken into account by employing the mean mixed concentration, $C_{0}$ (\% volume), which is defined as:

$$
C_{0}=100 \times \frac{Q_{\mathrm{CO}_{2}}+C_{a i r} Q_{\mathrm{aur}}^{\cdot}}{Q_{\mathrm{aur}}^{\dot{2}}}
$$

Where $Q_{\mathrm{CO} 2}$ and $Q_{\mathrm{air}}$ are the tracer and total fluidization air flow rates and $\mathrm{C}_{\mathrm{air}}$ (\% volume) is the concentration of $\mathrm{CO}_{2}$ in atmospheric air. The concentration of $\mathrm{CO} 2$ in the atmosphere was determined using the IR analyzers. $\mathrm{C}_{0}$, is the uniform concentration that would be attained if the riser were infinitely tall. The value of $\mathrm{C}_{0}$ was well above the normal concentration of $\mathrm{CO}_{2}$ in atmospheric air.

\subsubsection{The Tracer Gas Probe System}

Typically tracers are often used to obtain information on the mixing characteristics or residence time distribution of a reactor (Louge et al, 1997). By definition, a tracer is a material used to represent the flow properties of the substance into which it is injected. Ideally, a perfect 
tracer should have exactly the same flow properties as the substance it represents but at the same time should bear a characteristic which can be easily detected by an analytical instrument (Nauman and Buffham, 1983).

For studying the solids and gas mixing in fluidized beds, different types of solid and gas tracers have been used. The substances such as sodium chloride $(\mathrm{NaCl})$, ferromagnetic particles, fluorescent dye and irradiated sand have been utilized successfully as solid tracers in CFB risers (Werther and Hirschberg, 1998). Similarly, gases such as argon, helium, $\mathrm{CO}_{2}$, hydrogen, methane and ozone have been very commonly employed in gas mixing studies in CFB risers. Almost any gas or volatile liquid can be used as a tracer if there is a suitable detector. Linearity, detectability, and adsorption characteristics of the tracer gas on the solids particles should be considered. Namkung and Kim (1998) showed the effects of the adsorption on gas backmixing in a lab scale $\mathrm{CFB}$ when $\mathrm{CO}_{2}$ gas was used as the tracer gas with FCC particles.

In this study $\mathrm{CO}_{2}$ was chosen as the tracer gas because of its low cost, safety of operation and ease of analysis. The concentration of the $\mathrm{CO}_{2}$ gas can be accurately measured by an infrared gas analyzer.

\subsubsection{Gas Injection and Sampling System}

The schematic view of the tracer gas injection and sampling system is given in figure 2.8 and figure 2.9 respectively. The tracer gas injection apparatus consists of a cylinder in which compressed $\mathrm{CO}_{2}$ is at (100 psig), a dual stage pressure regulator, a mass flow controller to control and regulate its flow rate, and an injection probe. The tracer gas is injected at a continuous flow rate of 20 scfh. 


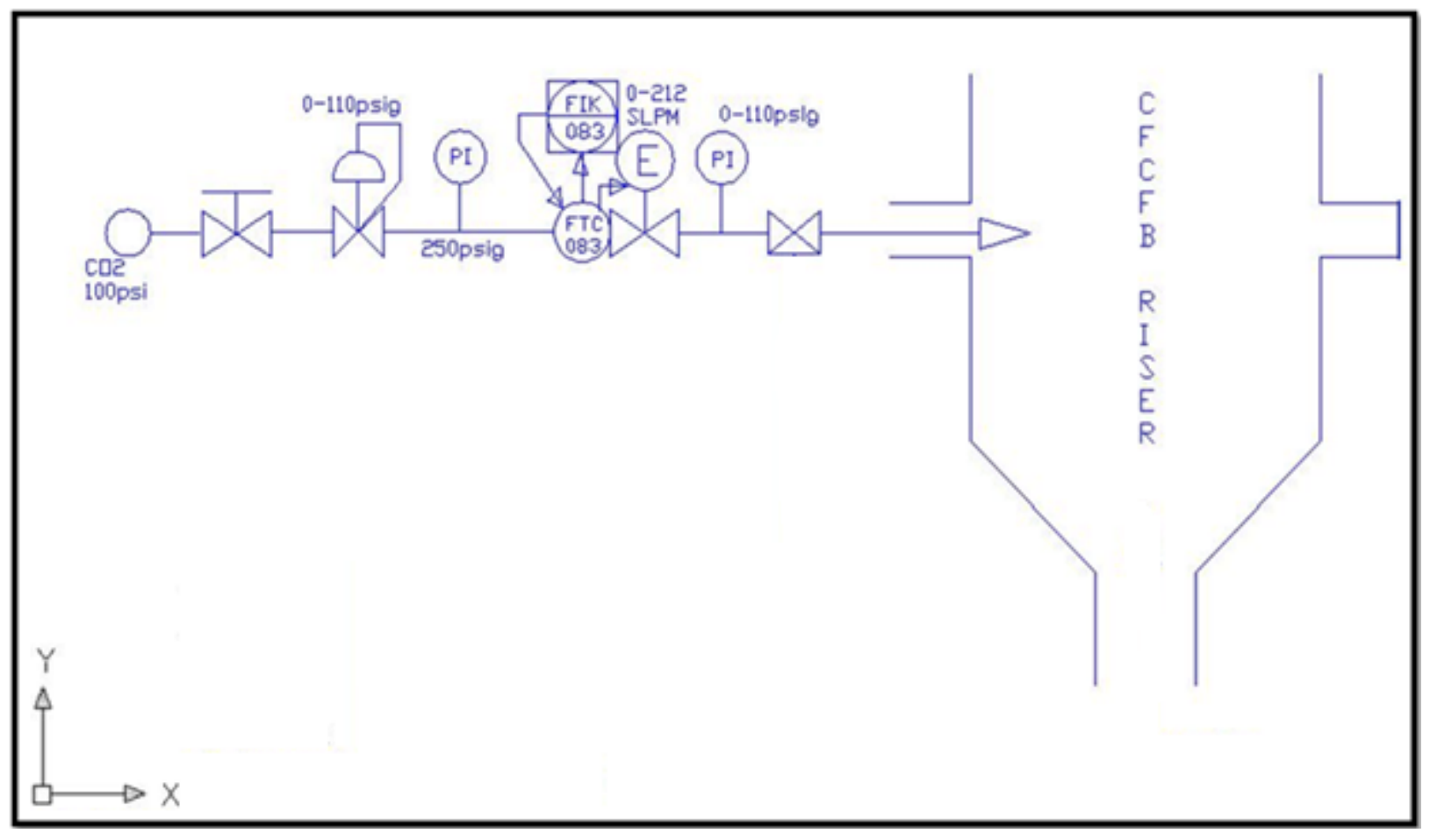

Fig2.8 Layout of the Gas Injection System

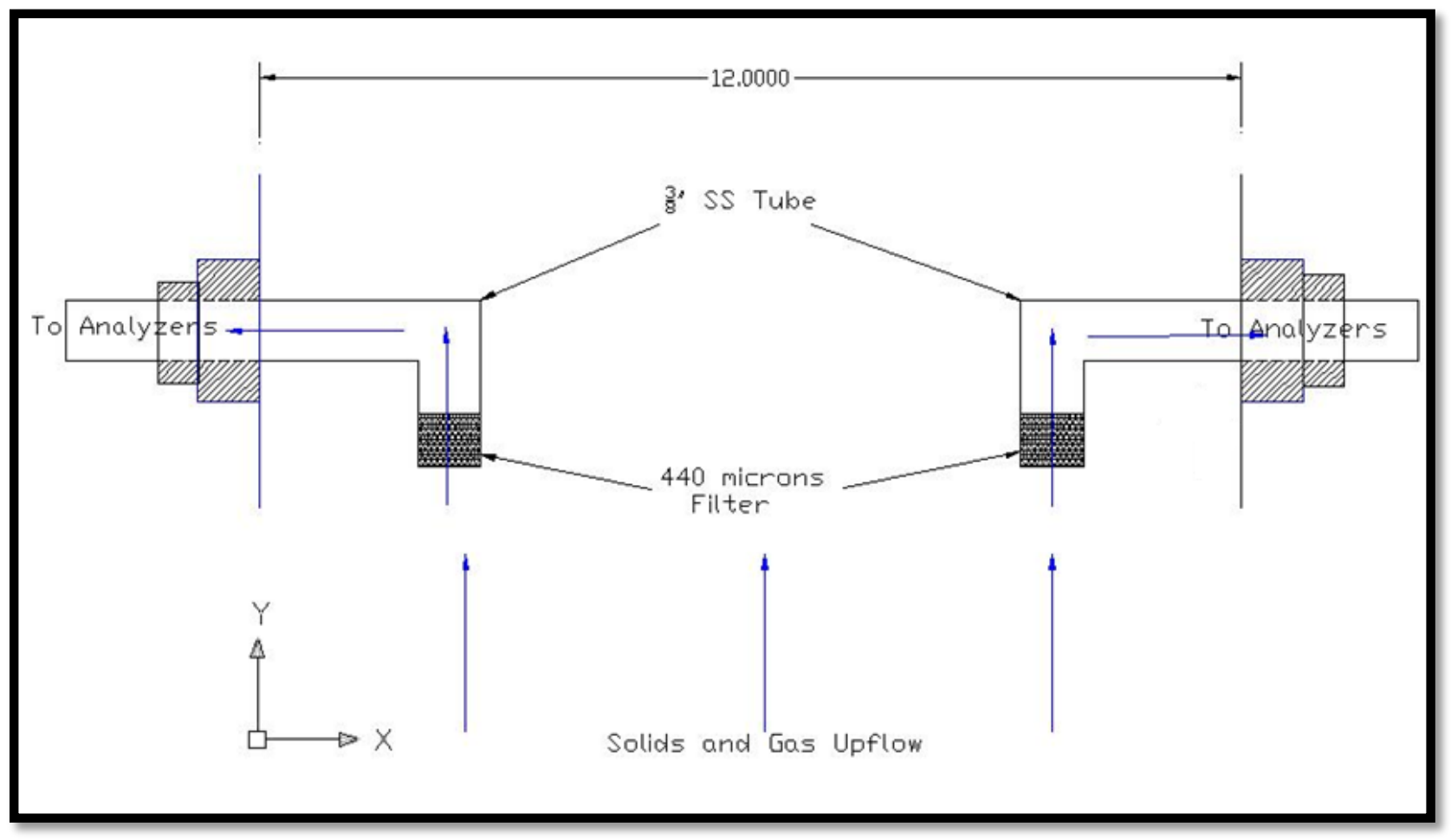

Fig2.9 Sampling Probe System 
The sampling line consists of a sampling probe and an infrared gas analyzer which was connected to the DAQ computer system. Nylon tubing, with a negligible permeability, is used throughout the injection and sampling system. The injection and sampling probes were made of 3.8 inch SS tubes, with a SS filter with pore size 440 microns was attached at the end of the probes to avoid any jet formation at the injection probe exit and prevent the solids from entering the sampling and injection lines. 


\section{Chapter III}

\section{Background Review - Gas Tracer Study}

\subsection{Gas Solid Fluidization Regimes}

Fluidized beds have enhanced mixing, uniform temperature distribution and higher contact efficiency when compared to other conventional reactors.

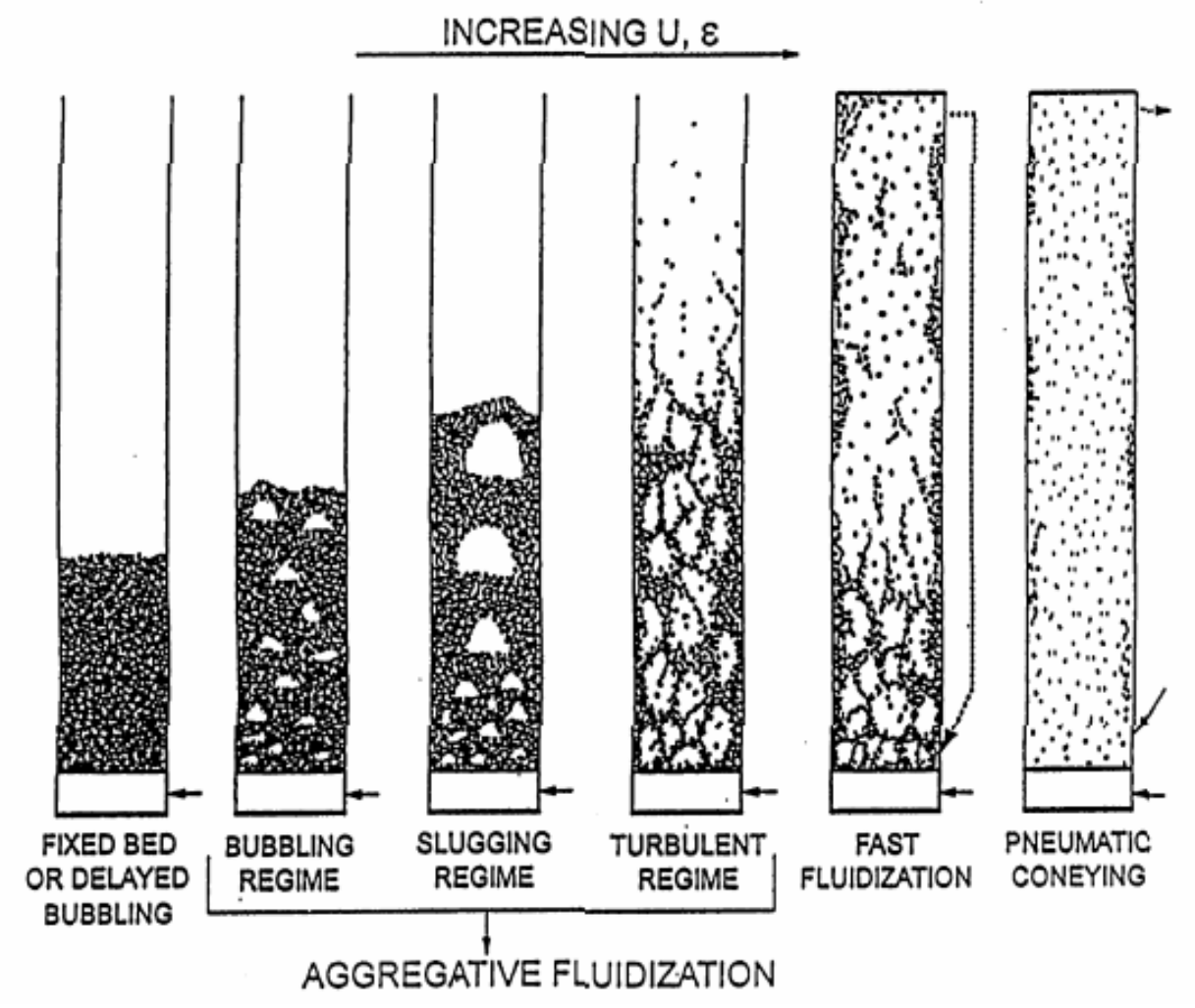

Fig. 3.1 Gas Solid Fluidization Regimes (Grace and Bi, 1997)

Figure 3.1 shows the fluidization regimes with increasing superficial gas velocity in fluidized beds (Grace and $\mathrm{Bi}, 1997)$. As the operational velocity is gradually increased in a bed filled with granular solids, the bed passes from the packed bed state to the fluidized state, once, the minimum fluidization velocity is 
exceeded. The minimum fluidization velocity, $\mathrm{U}_{\mathrm{mf}}$ is defined as the lowest gas velocity at which al1 the particles are suspended by gas. The onset of bubbling is indicated by the minimum bubbling velocity $U_{m b}$ the gas velocity at which the bubbles first appear in the bed. For beds of coarse particles, the minimum fluidization and the minimum bubbling velocities are usually close to each other whereas for the beds of fine particles a homogeneous bubble-free fluidization is bubble-free fluidization is observed between the minimum fluidization and minimum bubbling velocities.

When the minimum bubbling velocity is exceeded, gas bubbles form above the distributor plate and they grow as they rise towards the surface of the bed mainly due to coalescence. The top surface of the bed is well defined with bubbles breaking through periodically. The bubble size and speed increases with increasing supeficial gas velocity. With a further increase in gas velocity the size of the bubbles becomes even bigger covering the cross section of the bed, causing a slugging regime. The top section of the bed rises and collapses periodically with a reasonable regular frequency.

A continued increase in the velocity may eventually show a change in the pattern in the bed expansion. At this stage, the bubble phase loscs its identity, due to rapid coalescence and break up. This results in violently active and highly expanded bed. Particles are thrown into the freeboard above the bed. The bed surface is not very clear. Such beds are said to be operated in turbulent regime. The transition from bubbling/slugging regime to turbulent regime is gradua 1 and 
spans a range of gas velocities which depend on the properties of gas and solids. also on equipment scale (Yerushalmi, 1986). Yerushalmi and his co-workers, measuring the pressure fluctuations, were the first to characterize the transition to turbulent fluidization by two velocities $U_{\mathrm{c}}$ and $\mathrm{U}_{\mathrm{k}}$ (Yerushalmi, 1986). They proposed that the former marks the onset of the transition with a peak in the amplitude of the pressure fluctuations and the latter one shows the end of the transition with a leveling off in the amplitude of the pressure fluctuations. There is now a general agreement on $\mathrm{U}_{\mathrm{c}}$ however $\mathrm{U}_{\mathrm{k}}$ is the subject of continuing debate and discussions (Rhodes, 1996).

The turbulent regime extends to the so-called transport velocity, $U_{\text {tr. }}$ As the transport velocity is approached there is a sharp increase in the rate of particle carryover. and in the absence of solids recycle, the bed would empty rapidly. Beyond the transport velocity, particles fed to the bottom of the column or vesse 1 traverse it in fully entrained transport flow, and the concentration or density of the resulting suspension depends not only on the velocity of the gas but also on the flow ratte of solids. If the solids are fed to the column to its bottom via externa 1 cyclones and a standpipe then it is possible to maintain in the column with relatively large solids concentration typical of the fast-fluidization regime. The fast-bcd condition is also marked by aggregation of the particles in clusters and strands which break apart and reform in rapid succession, extensive back-mixing of solids, and slip velocities that are in order of magnitude greater thon the terminal velocities of the individual particles. 
The transition from the fast fluidization to the pneumatic transport regime is marked by the disappearance of a dense phase region of relatively high density and large amplitude pressure fluctuations at the bottom of the riser. In this regime, there is no axial variation of solids concentration except in the bottom acceleration section. However, some particle strands may still be identified near the wall (Grace, 1997).

Recently, new experimental evidences have shown the presence of a new flow regime occurring at high solids mass flux values $\left(\mathrm{G}_{\mathrm{s}}>250 \mathrm{~kg} \cdot \mathrm{m}^{2} \mathrm{~s}\right)$ and high superficial gas velocities (Grace et al., 1999). This new flow regime is named as dense suspension up-flow (DSU) by Grace et al., (1999). In this new flow regime, the solids no longer flow downward near the wall but they move upward and a coreannular structure does not exist. Clearly, more research is needed in this area. Boundaries for various flow regimes have been proposed in published literature. Grace et al. (1999) suggested a correlation to predict the onset of the DSU flow regime based upon the fully developed region of the riser. This correlation is given in equation (1) below;

$$
U_{D S U}=0.0113 G_{S}^{1.192} \rho_{g}^{-1.064}\left[\mu_{g} g\left(\rho_{p}-\rho_{g}\right)\right]^{-0.064}---(1)
$$

Gupta and Berruti (1998) also proposed the following correlation to predict the transition from core annulus to the DSU regime for group A particles. 


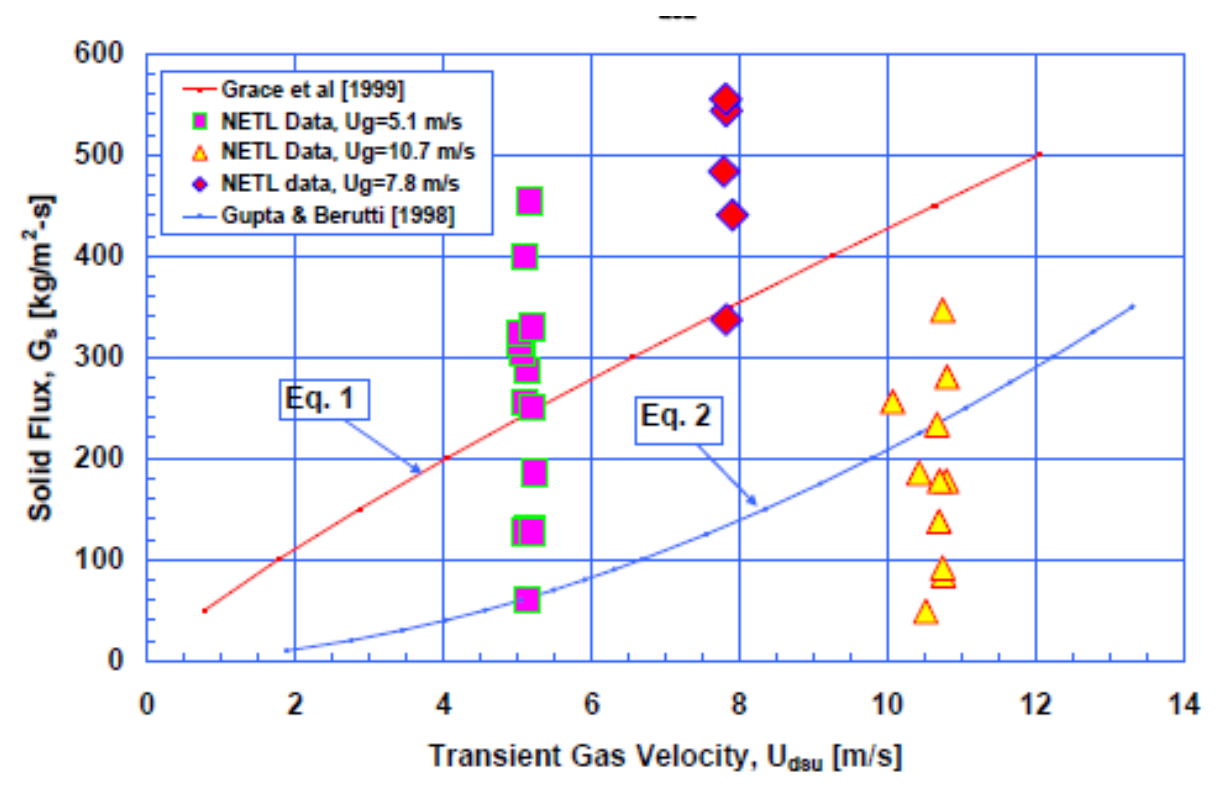

Figure3.2. Comparison of prediction of onset of DSU flow regime from correlations of Grace et al, (1999) and Gupta and Berruti (1998).

$$
U_{D S U}=12.55\left(\frac{G_{S}}{U_{t} \rho_{S}}\right)^{0.55} A r^{0.36}\left(\frac{\mu_{g}}{d p \rho_{g}}\right)---(2)
$$

In the context of the description of the gas-solid fluidization regimes, in a circulating fluidized bed several regimes can co-exist, simultaneously. The bottom dense region of industrial CFB combustors are usually in bubbling regime in contrast to laboratory scale circulating fluidized beds whose bottom parts operate mostly in turbulent regime (Svensson et al. 1996; Leckner, 1988) whereas the riser upper part is in fast fluidization or pneumatic transport regimes. It is believed that the DSU does not occur in the dense region which is the focus of this research work. 


\subsection{Flow Behavior and Regime Transition in a high density circulating fluidized bed riser}

Consider a steady and isothermal gas - solids flow in the riser, as shown in figure 3.3. If we ignore, the solids deceleration near the top exit of the riser and the intensive mixing regime near the bottom of the riser where solids and gas are introduced, the main part of the riser would consist of three regimes, namely, the dense regime, acceleration regime and dilute regime. The classification of these regimes is based on the hydrodynamic characteristics of solids flow and operating conditions. 


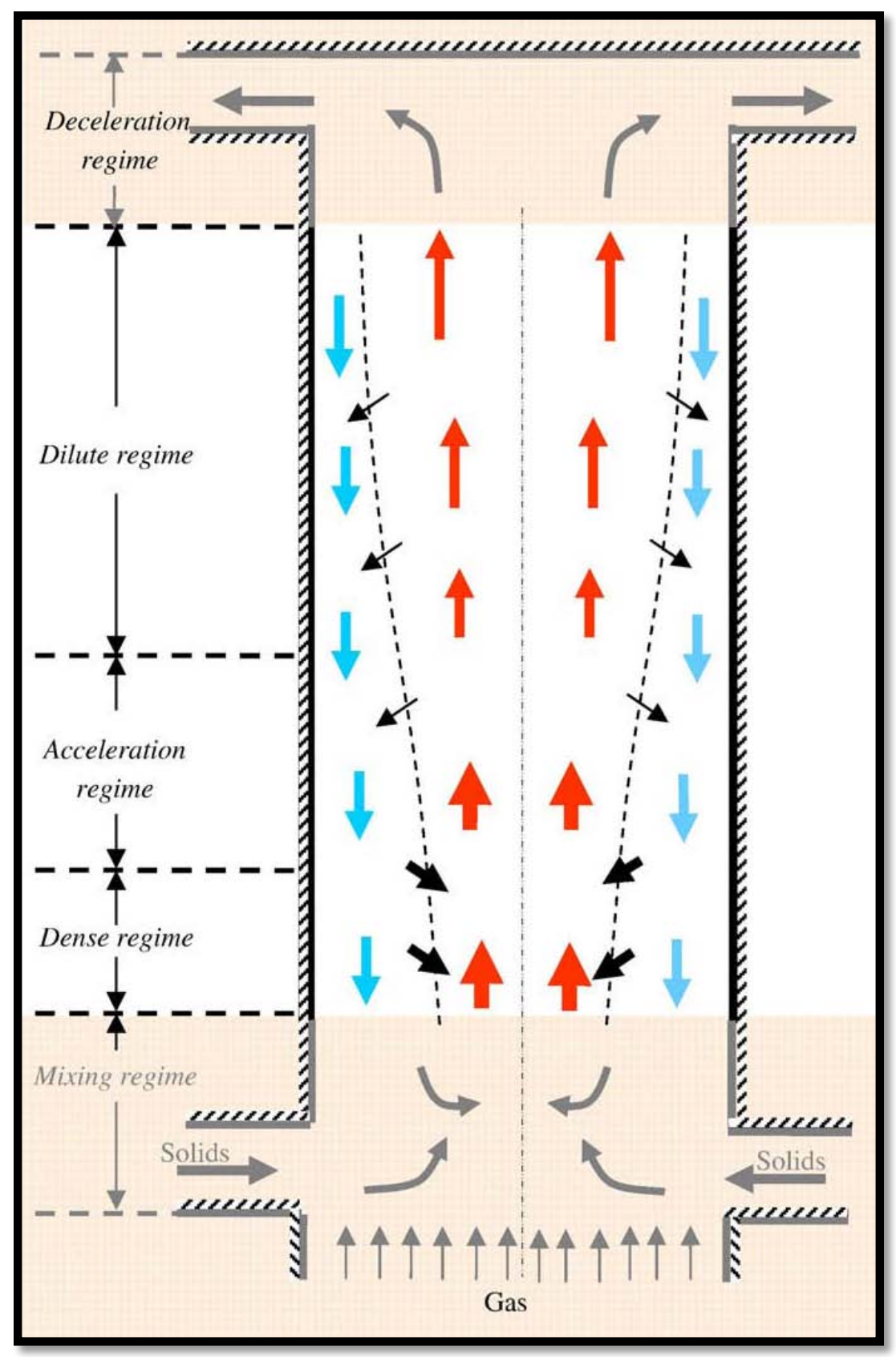

Fig3.3. Flow Structure in a Solid - Gas Riser

\subsection{Contemporary Work Done}

Sterneus et.al, (2000) carried out steady-state tracer-gas measurements were carried out in a circulating fluidized-bed (CFB) boiler and in a cold experimental (CFB) unit. The cold unit is $8.5 \mathrm{~m}$ tall and has a cross-section of $0.12 \times 0.70 \mathrm{~m}$. The 
bed material was the same as in the boiler. Gas velocity and solids mass flux could be changed independently, and therefore it was possible to study the influence of gas velocity and solids concentration separately. The main focus of their work was on the radial spread of gas in the core region of the transport zone (above 5.85m) under different operating conditions. Helium (He) was used as tracer gas, and the local He-concentration was determined by a mass spectrometer. The fluidization velocity ranged from 1.2 to $4.3 \mathrm{~m} / \mathrm{s}$ in the boiler and from 1 to $6 \mathrm{~m} / \mathrm{s}$ in the cold unit.

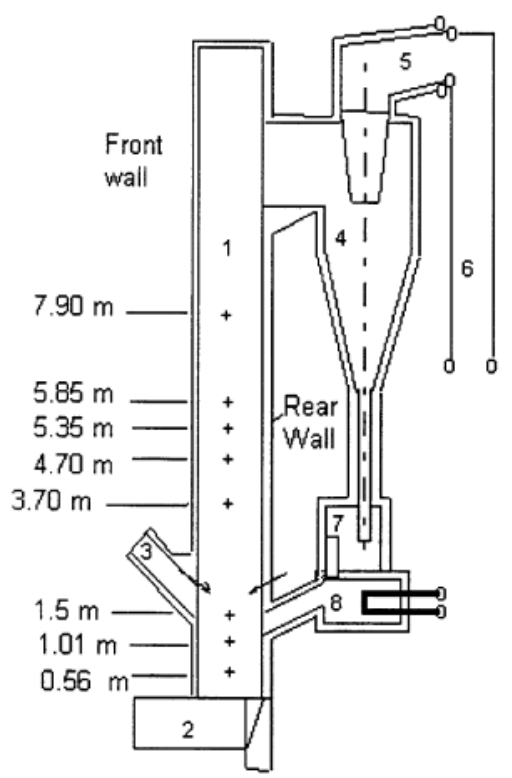

Figure3.4 Principle outline of the 12 MW boiler (1) Furnace, (2) air plenum, (3) fuel feed chute, (4) cyclone, (5) exit duct, (6) convection pass, (7) particle seal and (8) particle cooler, $(\rightarrow)$ Secondary air nozzles, $(+)$ measurement holes used in this study. Sterneus et.al, (2000) The bed material was silica sand with an average particle diameter of $0.32 \mathrm{~mm}$ in both units. Measurements were also carried out in the bottom bed (3.70 to $5.85 \mathrm{~m}$ ) 
and splash zone (0.56 to $1.5 \mathrm{~m})$ of the boiler for comparison with those of the transport zone.

Figure 3.4 shows the principle outline of the $12 \mathrm{MW}$ boiler used by the authors for their work.

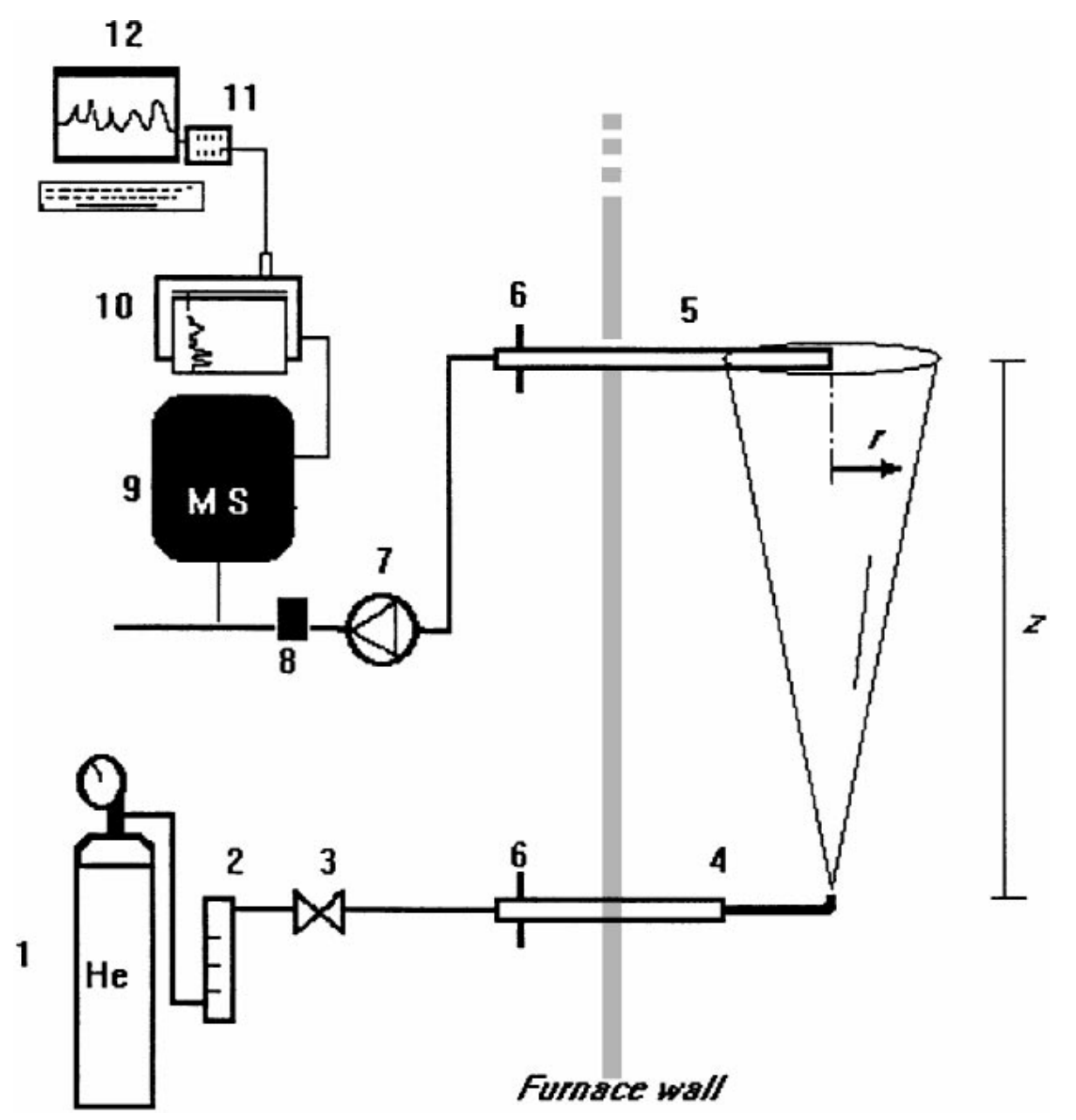

Figure3.5. Schematic outline of the measurement equipment (1) Helium tube, (2) flow meter, (3) valve, (4) injection probe, (5) sampling probe, (6) cooling water, (7) pump, (8) dryer (blue gel), (9) mass spectrometer, (10) plotter, (11) AD-converter and (12) computer. Sterneus et.al, (2000) 
A schematic outline of the measurement equipment is given in figure 3.5. Tracer gas (helium) was continuously introduced from a bottle (1) by an injection probe (4)

In the first series of measurements, two injection probes (water cooled) were used. The sampling probe (5) $(2.3 \mathrm{~m}, 25 \mathrm{~mm}$ ID) was the same in all measurements. The mass spectrometer (9) gave a voltage signal corresponding to the Heconcentration of the sampled gas. The original signal was amplified and a measurement computer (12) recorded the output signal (0\}10 V) during 90$\} 180 \mathrm{~s}$ with a frequency of $10 \mathrm{~Hz}$.

During the core measurements, the tracer gas was introduced at the centre line and a radial (horizontal) concentration profile was measured downstream of the injection level. Initial tests showed that there was no backmixing in the core.

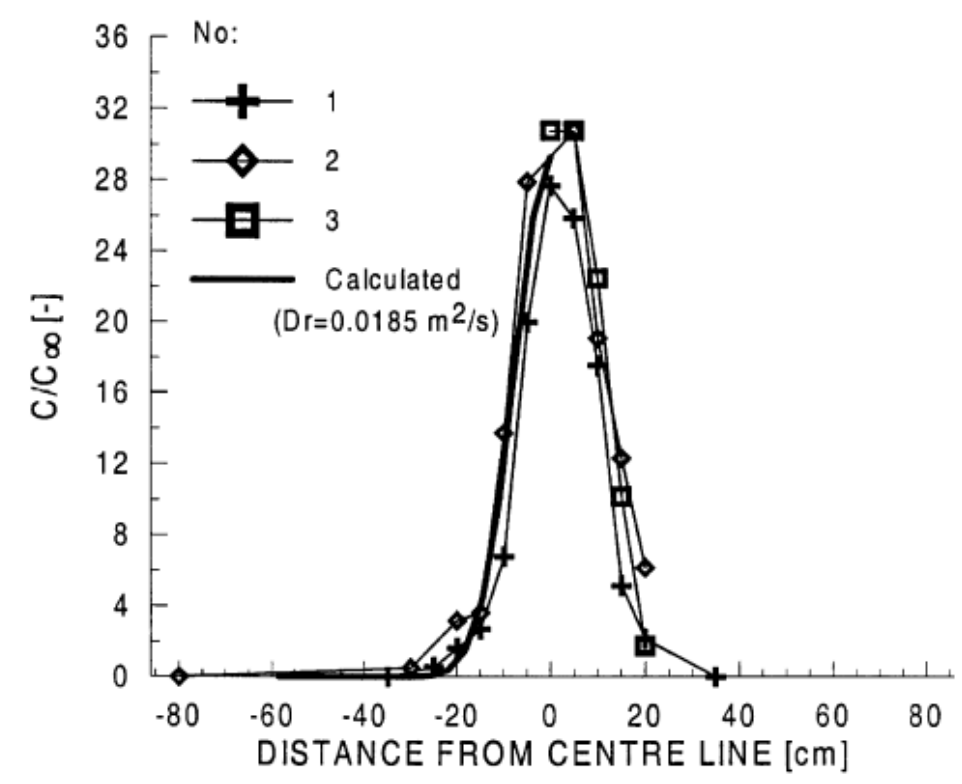

Figure3.6. Gas concentration profiles measured at three different occasions. $U=2.6$ $\mathrm{m} / \mathrm{s}, \mathrm{z}=0.65 \mathrm{~m}, \mathrm{H}_{\text {inj }}=4.7 \mathrm{~m}$. Sterneus et.al, (2000) 


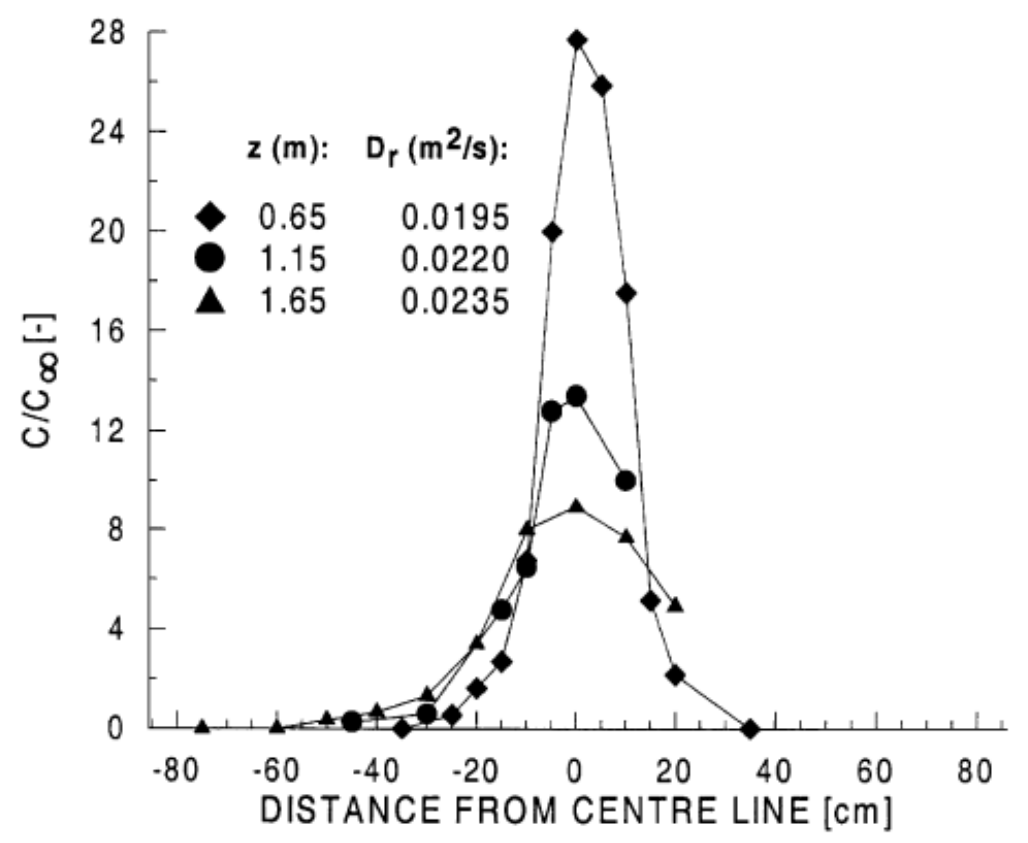

Figure3.7. Tracer gas concentration profiles measured at ; $\mathrm{U}=2.6 \mathrm{~m} / \mathrm{s}$ and different vertical measuring distances. Sterneus et.al, (2000)

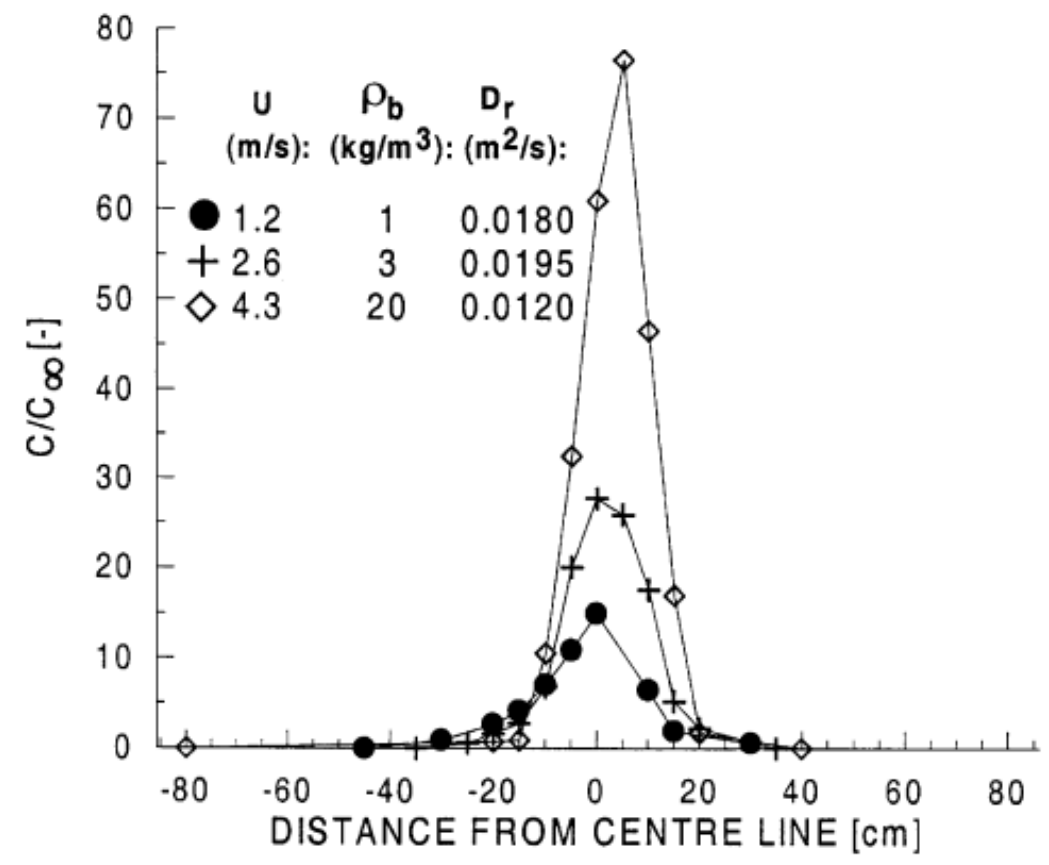

Figure3.8. Gas concentration profiles obtained at three operating conditions $(\mathrm{U}=$ $1.2,2.6$ and $4.3 \mathrm{~m} / \mathrm{s}$ ) and, $z=0.65 \mathrm{~m}$. Sterneus et.al, (2000) 
As seen from figure 3.6 for a center line injection the time averaged tracer gas profile is parabolic or leptokurtic as expected. As the distance from the injection point increases the profile starts transitioning from a leptokurtic to a platykurtic profile as shown in figure 3.7. And for a constant elevation, as the superficial gas velocity increased the leptokurtic tracer gas profile started transitioning to a platykurtic profile as shown in figure 3.8.

Zheng et al, (1996) carried out experiments on radial gas dispersion in a cold model CFB using tracer gas technique. The test system is shown in figure 3.9. It consists of a riser with $0.102 \mathrm{~m}$ I.D., $5.25 \mathrm{~m}$ in height and a downcomer with $0.154 \mathrm{~m}$ I.D., two staged cyclones and an L-Valve.

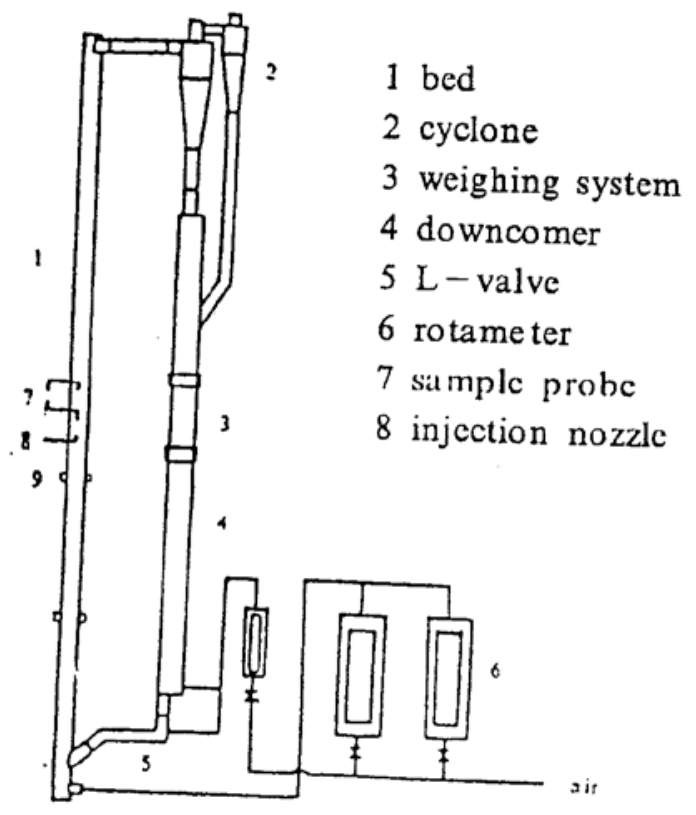

Figure3.9. Schematic diagram of the experimental system Zheng et al, (1996)

The tracer gas injection and sampling system is shown in figure 3.10 and are designed to obtain the radial dispersion coefficient $\mathrm{D}_{\mathrm{r}}$. Carbon Dioxide tracer gas is 
injected continuously through a nozzle into the center of the bed, $2.3 \mathrm{~m}$ above the circulating solid inlet. Sampling points are located at different levels above the injection point.

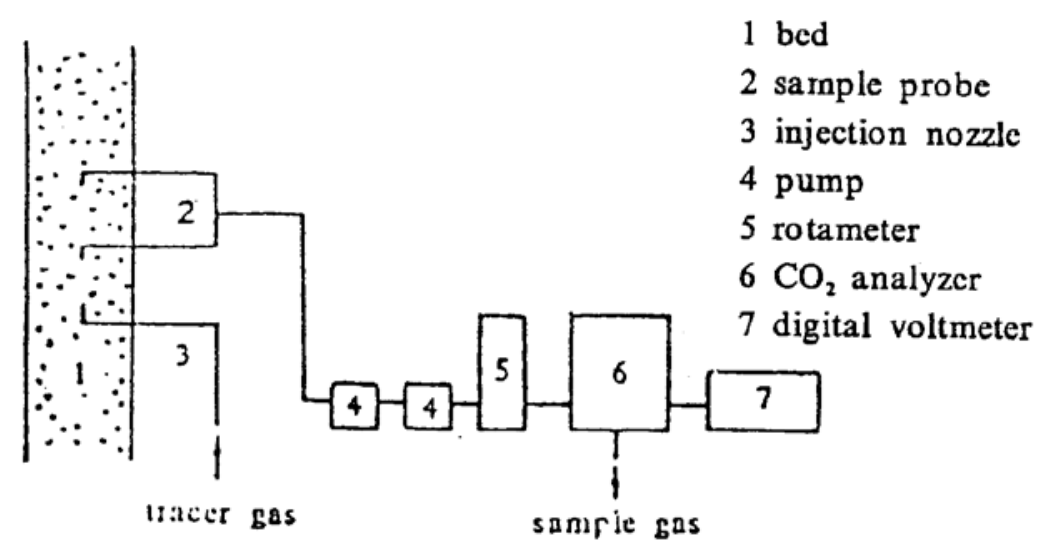

Figure3.10. Gas Tracer System Zheng et al, (1996)

The test materials were resins and quartz sands with density of $1392 \mathrm{~kg} / \mathrm{m}^{3}$ and $2560 \mathrm{~kg} / \mathrm{m}^{3}$ respectively. The experiments were conducted with four kinds of particles to measure the radial concentration of the tracer gas in the bed under various gas velocities $\mathrm{U}_{\mathrm{f}}$ and solids flux $\mathrm{G}_{\mathrm{s}}$ with and without secondary air injection. The mean diameters of resins were $0.567 \mathrm{~mm}\left(1^{\#}\right)$ and $0.701 \mathrm{~mm}\left(2^{\#}\right)$ and of sands were $0.364 \mathrm{~mm}\left(3^{\#}\right)$ and $0.570 \mathrm{~mm}\left(4^{\#}\right)$. The effect of $\mathrm{U}_{\mathrm{f}}$ and $\mathrm{G}_{\mathrm{s}}$ on the gas dispersion coefficient is shown in figure 3.11. From the curves it was seen that $\mathrm{U}_{\mathrm{f}}$ and $\mathrm{G}_{\mathrm{s}}$ affect Dr unmonotonously. 


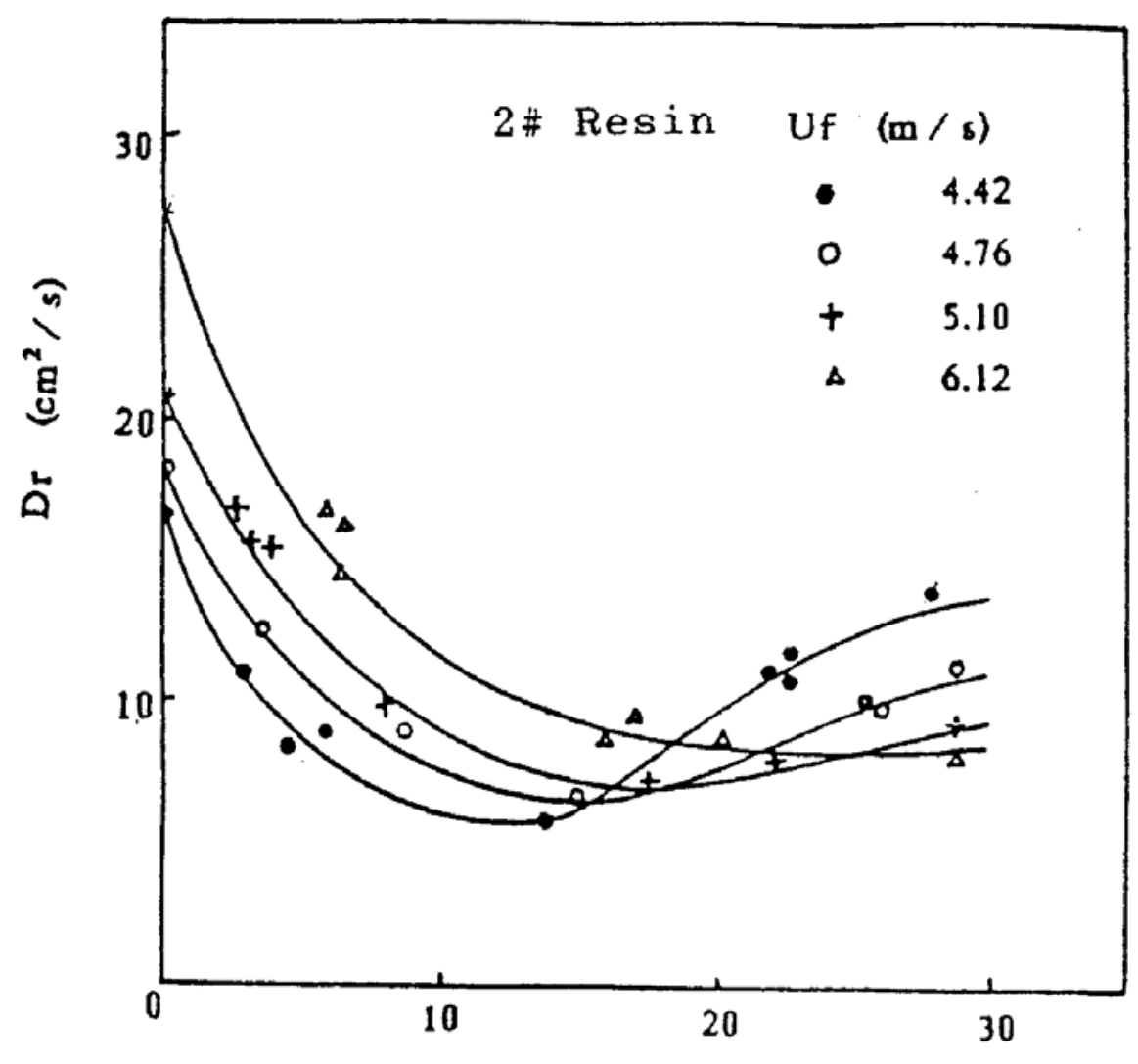

\section{Gs $\left(\mathbf{k g} / \mathrm{m}^{2} \mathrm{~s}\right)$}

Figure3.11. Radial Dispersion Coefficient at Different Gas Velocity and Solids Mass Flux. Zheng et al, (1996)

The effect of the gas velocity on the radial dispersion coefficient is not monotonous. There is a certain value of $\mathrm{G}_{\mathrm{s}}$, acting as the threshold. When the $\mathrm{G}_{\mathrm{s}}$ is less than the threshold value, $D_{r}$ increases with the increase in $U_{f}$. When the $G_{s}$ is higher than the threshold value, $D_{\mathrm{r}}$ decreases with the increase in $\mathrm{U}_{\mathrm{f}}$. The effect of $\mathrm{G}_{\mathrm{s}}$ on $\mathrm{D}_{\mathrm{r}}$ is also not monotonous. $\mathrm{D}_{\mathrm{r}}$ first decreases to a minimum value with the increase in $\mathrm{G}_{\mathrm{s}}$ and then increases along with the increase in $\mathrm{G}_{\mathrm{s}}$. When the gas velocity remains constant, a minimum $\mathrm{D}_{\mathrm{r}}$ exists in the curves shown in figure 3.11. 
Experiments were carried out Kim et al., (2004) using a Plexiglas riser (0.203m id. by $5.9 \mathrm{~m}$ height) coupled to a cold model (Knapper et al., 2002) commercial fluid cokers, as shown in figure 3.12. The solids in this study were FCC particles of mean diameter $70 \mu \mathrm{m}$ and density $1700 \mathrm{~kg} / \mathrm{m}^{3}$. The unit was operated at steady state, with solids flowing downwards through a standpipe and U-bend to the bottom of the riser. The overall solids circulation rate was controlled by a pinch valve at the location shown.

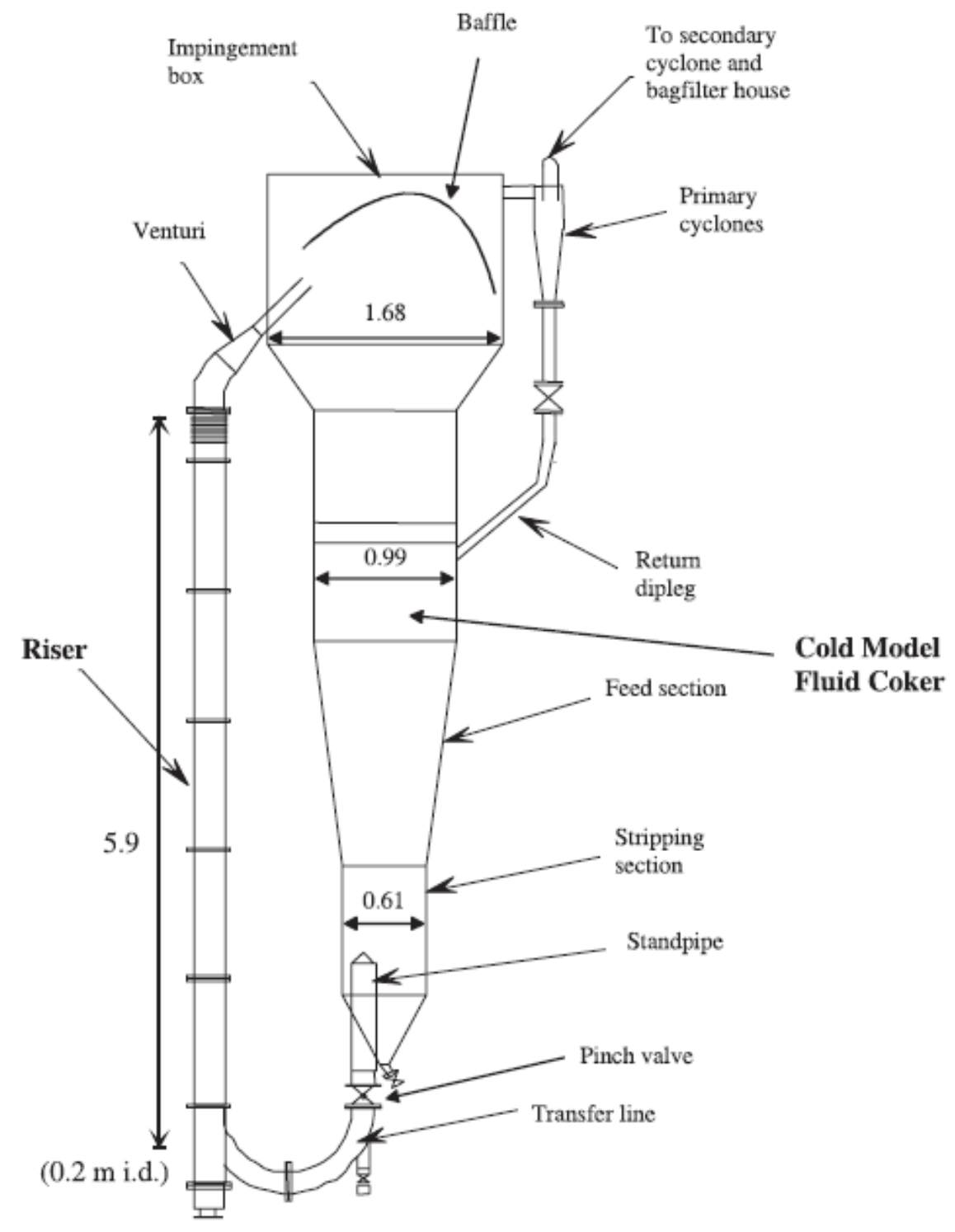


Figure3.12 Schematics of Experimental Apparatus (Kim et. al. 2004)

At the top of the riser, the gas and entrained particles passed through a venturi constriction after a $45^{\circ}$ bend before entering an impingement box. A baffle separator, located in the impingement box removed most of the particles from the air stream leaving the riser. The flow from the separator passed through primary cyclones and most particles returned to the fluidized return system through diplegs. A secondary cyclone and two bag filter houses in parallel captured any remaining particles in the exiting air stream.

An orifice meter was used to measure the gas flow rate in the riser. The airflow rate and superficial velocity $\left(\mathrm{U}_{\mathrm{g}}\right)$ were calculated from the pressure drop $(\Delta \mathrm{P})$ across the orifice meter. The solids mass flux $\left(\mathrm{G}_{\mathrm{s}}\right)$ through the riser was calibrated by monitoring the $\Delta \mathrm{P}$ across the venturi constriction at the top of the riser, while simultaneously measuring the solids mass flux in the standpipe using a fiber optical velocitimeter probe to determine the solids void fraction and velocity. Pressure taps were mounted flush with the column wall and covered with a screen to prevent particle leakage. Pressure transducers (Omega, PX140) were connected to pressure taps along the column height to measure $\Delta \mathrm{P}$ between different locations. The signals from the pressure transducers were amplified and sent via an A/D converter to a personal computer for recording. Apparent solid holdups $\left(\varepsilon_{\mathrm{s}}\right)$ were estimated from the measured pressure drops (Issangya et al., 1997, 1999; Namkung et al., 1999), i.e. from

$$
\varepsilon_{\mathrm{S}}=\Delta \mathrm{P} / \rho_{\mathrm{p}} \mathrm{g} \Delta \mathrm{z}
$$


A schematic diagram of the momentum probe is shown in figure 3.13 .

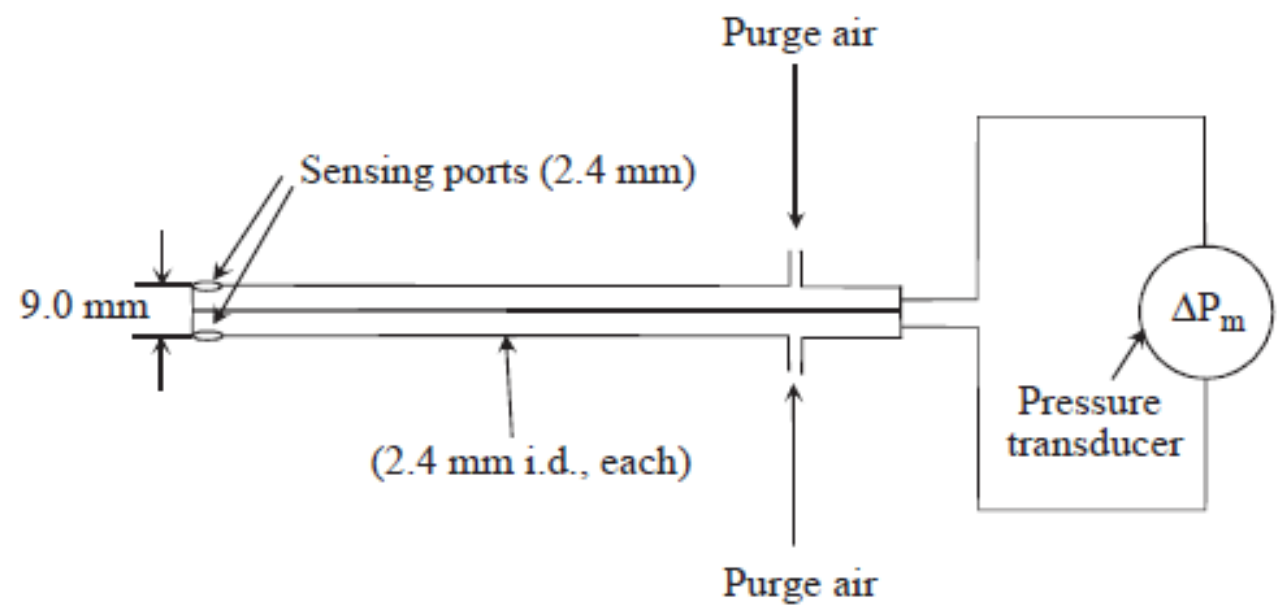

Figure3.13. Schematic diagram of the momentum probe (Kim et al, 2004)

Interaction between the gas-solid suspension and the probe results in momentum transfer to the gas. The momentum was measured by means of the dynamic pressure signals. The probe is similar to the momentum probe of Zhang et al. (1995, 1997). It consists of two stainless-steel tubes (both $2.4 \mathrm{~mm}$ id.), small enough that interference with the flow is minimized. The end $(2.4 \mathrm{~mm}$ id.) of one tube faces upstream through a right angle, while the other end (2.4 $\mathrm{mm}$ id.) is directed downstream with an identical shape. The probe was traversed horizontally so that measurements could be made at different radial positions. A preliminary test was carried out to determine the purging air velocity $\left(\mathrm{U}_{\mathrm{pa}}\right)$ through the momentum probe. Air-purging at high pressure (4 bar air) and $U_{\mathrm{pa}}=10.5 \mathrm{~m} / \mathrm{s}$ was chosen to prevent entrained particles from blocking the tube holes and to minimize the effects of purging air on riser gas flow (Rhodes et al., 1998; Kim et al., 2001). A pressure transducer (Omega, 140X) and data acquisition system recorded the instantaneous pressure signals. In this way, the measured time-mean pressure 
drop, $\Delta \mathrm{P}_{\mathrm{m}}$, across the two tips should correspond to the average linear momentum flux of the gas-solid suspension flow, neglecting the static pressure difference between the two ports (Bai et al., 1995; Issangya et al., 1997; Kim et al., 2001):

$$
\Delta \mathrm{P}_{\mathrm{m}}=\alpha\left(\rho_{\mathrm{g}} \overline{\varepsilon \mathrm{U}_{\mathrm{g}}^{2}}+\rho_{\mathrm{p}} \overline{\varepsilon_{\mathrm{s}} \mathrm{V}_{\mathrm{s}}^{2}}\right) \cong \alpha \rho_{\mathrm{p}} \overline{\varepsilon_{\mathrm{s}} \mathrm{V}_{\mathrm{s}}^{2}}---(1)
$$

where $\alpha$ is a momentum transfer coefficient. Since the solids density is usually more than 3 orders of magnitude greater than the gas density, the gas momentum flux term (first term on the right-hand side of Eq. (1)) can reasonably be ignored with a relative error of less than 5\% (Bai et al., 1995). Figure 3.14 shows axial profiles of solids apparent holdup in the riser as a function of the solids mass flux $\left(\mathrm{G}_{\mathrm{s}}\right)$ at a riser gas velocity $\left(\mathrm{U}_{\mathrm{g}}\right)$ of $6.0 \mathrm{~m} / \mathrm{s}$.

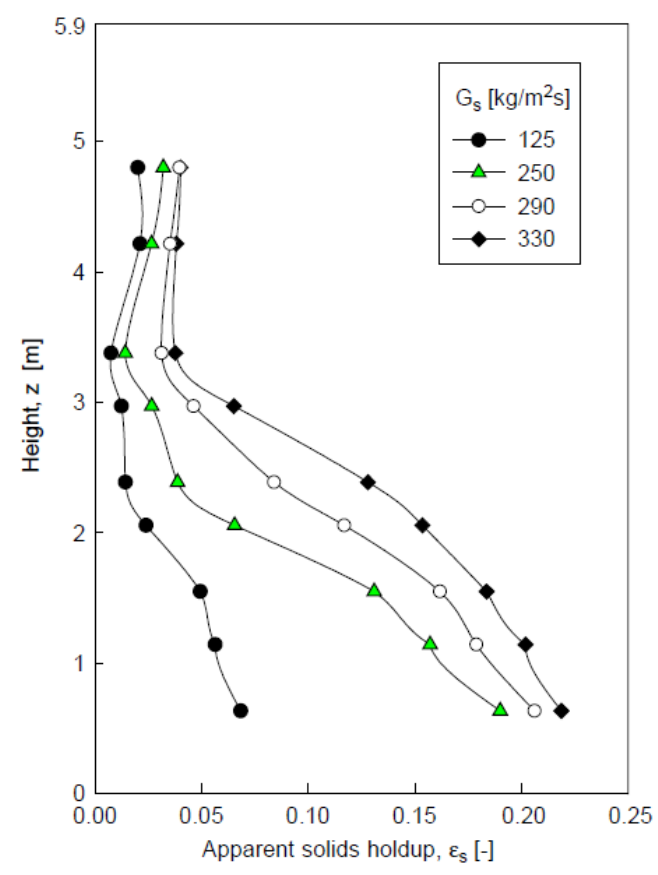

Figure3.14. Axial Profile of cross sectional mean solids holdup for various solids mass fluxes at $U_{g}=6 \mathrm{~m} / \mathrm{s}$ (Kim et.al, 2004) 
Solids holdup $\left(\varepsilon_{\mathrm{s}}\right)$ decreases sharply with increasing height at each $\mathrm{G}_{\mathrm{s}}$. With increasing $\mathrm{G}_{\mathrm{s}}, \varepsilon_{\mathrm{s}}$ increases and the shape of the axial distribution profiles changes to an S-shape with a dense phase at the bottom and a relatively dilute region in the top section. These trends are similar to those in several previous studies of high density riser flows (Malcus et al., 2002; Karri and Knowlton, 2002; Manyele et al., 2002).

On the other hand, Issangya et al. (1999) found the dense suspension to persist over the entire riser height at high $\mathrm{G}_{\mathrm{s}}$. Pressures in the CFB loop must be balanced for stable steady-state operation. The pressure drop in the standpipe or return system provides the driving force for solids flow and solids holdup in the riser (Kim and Kim, 2002). The different profiles reported by Issangya et al. (1999) likely arose because they adopted a dual CFB system with a standpipe whose height was larger than that of the riser and with a sufficient solids inventory that they could obtain high pressure-drops and $\varepsilon_{\mathrm{s}}$ over the entire riser. Different exit geometries may also have contributed to different axial profiles of solids holdup reported in the literature. 


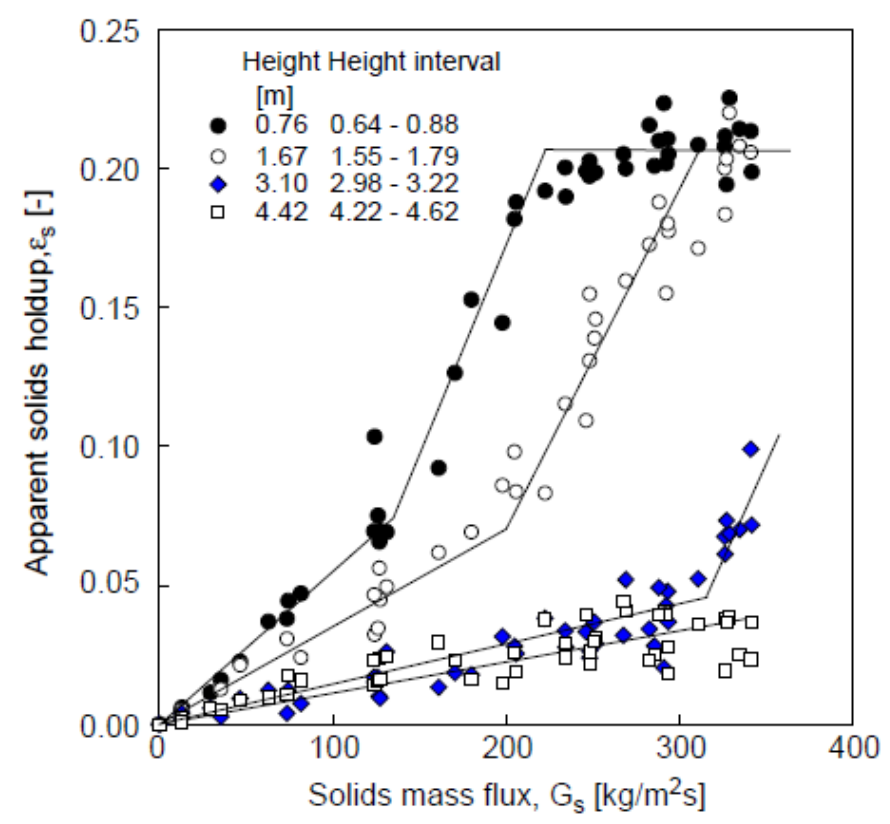

Figure3.15. Effect of solids mass flux on solids hold up for various heights in the riser at $\mathrm{U}_{\mathrm{g}}=6 \mathrm{~m} / \mathrm{s}$ (Kim et.al, 2004)

The effect of solids mass flux on solids holdup at several heights along the riser is shown in figure 3.15 for a gas velocity of $6 \mathrm{~m} / \mathrm{s}$. A dense region forms in the lower section of the riser, with a steep increase in $\varepsilon_{\mathrm{s}}$ as $\mathrm{G}_{\mathrm{s}}$ increases. When Gs reaches about $200 \mathrm{~kg} / \mathrm{m}^{2} \mathrm{~s}, \varepsilon_{\mathrm{s}}$ at the lowest measurement level $(0.76 \mathrm{~m})$ reaches a constant value of about $0.21 ; \varepsilon_{\mathrm{s}}$ shows no further increase with increasing $\mathrm{G}_{\mathrm{s}}$, indicating that this location is in the high-density condition or dense suspension up flow regime (Issangya et al., 1999). With a further increase of $\mathrm{G}_{\mathrm{s}}$ above $200 \mathrm{~kg} / \mathrm{m}^{2} \mathrm{~s}$, the high-density condition was also reached at the next measurement level ( $\mathrm{z}=$ $1.67 \mathrm{~m})$. However, the two locations nearest the top $(\mathrm{z}=3.10$ and $4.42 \mathrm{~m})$ were still in the lean phase without a sharp increase of $\varepsilon_{\mathrm{S}}$ for the given $\mathrm{G}_{\mathrm{s}}$ range. These results indicate that the flow behavior and regime transitions are functions of height in the riser. 
Werther et.al (2001) conducted experiments to determine the solids mixing in the bottom zone of a circulating fluidized bed. The experiments were carried out in a cold model circulating fluidized bed depicted in figure 3.16. The riser has an inner cross-section of $0.3=1.0 \mathrm{~m}$ and a height of $8.5 \mathrm{~m}$. The off-gas is cleaned by two cyclones, the solids are returned into the riser at a height of $1 \mathrm{~m}$ within an angle of 458. The externally circulating solids mass flux is measured by a weighting section in the downcomer pipe.

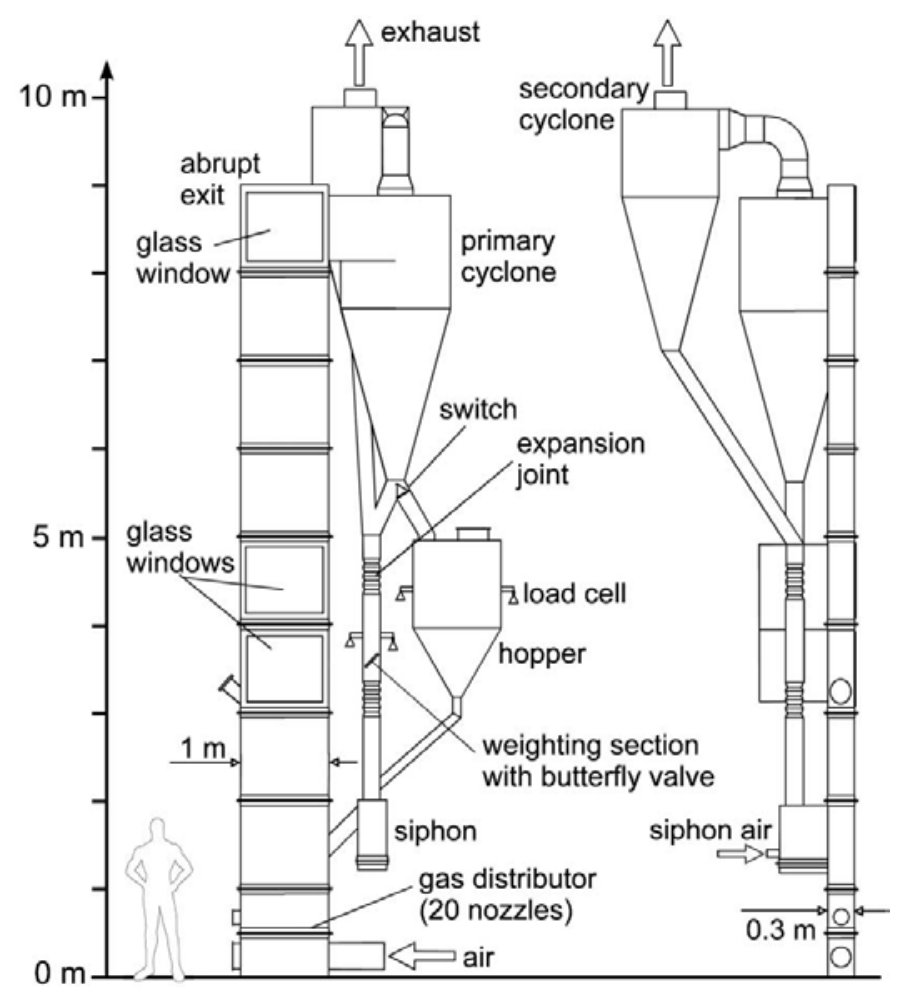

Figure3.16. Pilot plant CFB system Riser cross-section $0.3 \times 1.0 \mathrm{~m}$, height $\mathrm{H}=8.5 \mathrm{~m}$ Werther et.al (2001)

Quartz sand with a Sauter diameter $d_{p}$ of $150 \mu \mathrm{m}\left(\mathrm{U}_{\mathrm{mf}}\right.$ in air under ambient conditions $0.03 \mathrm{~m} / \mathrm{s}$ ) was used as bed material. Operating conditions, which are typical for CFB combustors, were chosen $\mathrm{u}=3 \mathrm{~m} / \mathrm{s}, \Delta \mathrm{P}_{\text {riser }}=10^{3} \mathrm{~Pa}, \mathrm{G}_{\mathrm{s}}=20 \mathrm{~kg} / \mathrm{m}^{2} \mathrm{~s}$ ) 
in the experiments. To investigate solids mixing pellets of solid carbon dioxide (dry ice) were fed over a limited time via a screw feeder into the bottom zone of the rectangular CFB riser, as shown in figure 3.17.

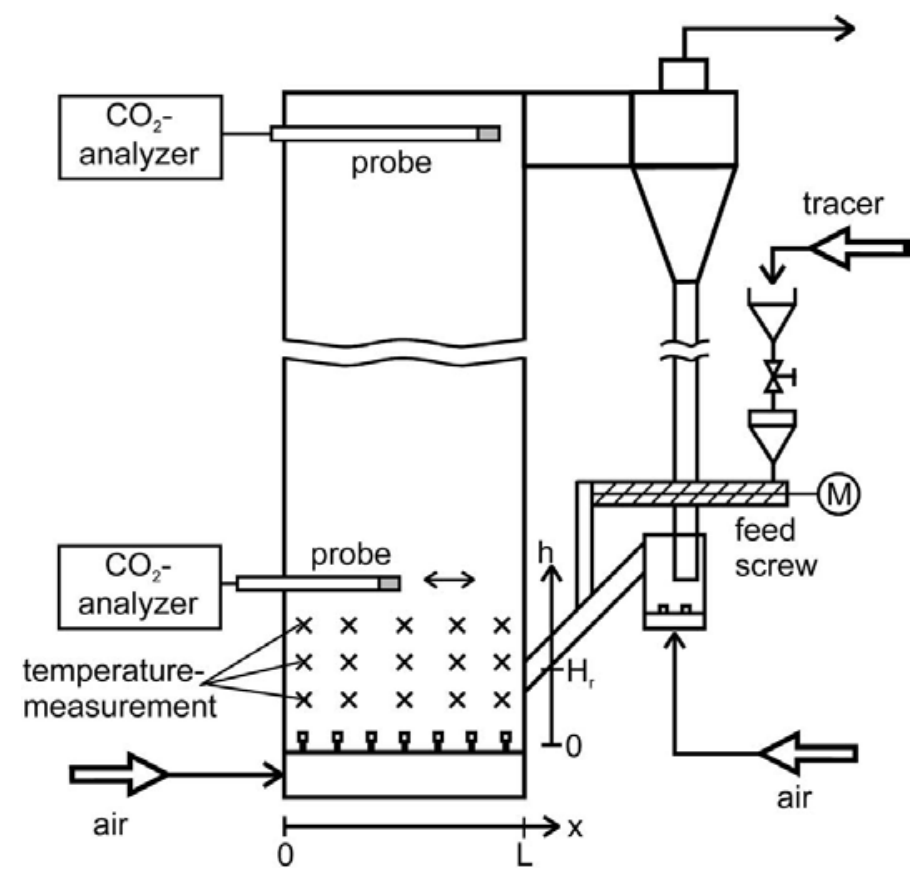

Fig3.17 Experimental setup for the investigation of solids mixing Werther et.al (2001)

The tracer was supplied at three different locations_-from the left side $(\mathrm{x}=$ $0 \mathrm{~m}, \mathrm{y}=0 \mathrm{~m}, \mathrm{~h}=0.35 \mathrm{~m})$, from the back into the center of the riser $(\mathrm{x}=0.5 \mathrm{~m}, \mathrm{y}=0 \mathrm{~m}$, $\mathrm{h}=0.35 \mathrm{~m})$ and into the solids return line, which ended just above the bottom bed at $(\mathrm{x}=1 \mathrm{~m}, \mathrm{y}=0 \mathrm{~m}, \mathrm{~h}=1 \mathrm{~m})$. The tracer particle feed rate was $135 \mathrm{~kg} / \mathrm{h}$ for about $120 \mathrm{~s}$.

The distribution of the tracer particles in the riser was monitored by two different methods (Bellgardt et al 1986). On one hand, the local concentration of the emanating $\mathrm{CO}_{2}$ gas was measured by collecting gas samples with a suction probe. On the other hand local bed temperatures were obtained with aid of a QuaT 
temperature measuring system (Heraeus Sensor) whose sensor is a piezoelectric resonator (Schaudel et al 1989The transition from solid carbon dioxide to gaseous carbon dioxide occurs at atmospheric pressure. The specific sublimation heat $\Delta \mathrm{H}$ is $25.23 \mathrm{~kJ} / \mathrm{mol}$ at a temperature of $194.67 \mathrm{~K}$.

Cylindrical pellets (Kühlhaus, Hamburg), having a constant diameter of 8 $\mathrm{mm}$ with a length up to $12 \mathrm{~mm}$, were used in the experiments. Horizontal profiles of the local carbon dioxide gas concentrations within the riser were measured along the $\mathrm{y}$ and $\mathrm{x}$-directions. In the measurements performed along the $\mathrm{y}$-direction of the riser, no concentration gradients were observed. Therefore, the horizontal profiles in the $\mathrm{x}$-direction were measured at the center line at ys $0 \mathrm{~m}$ at a height of $\mathrm{h}=0.56 \mathrm{~m}$.

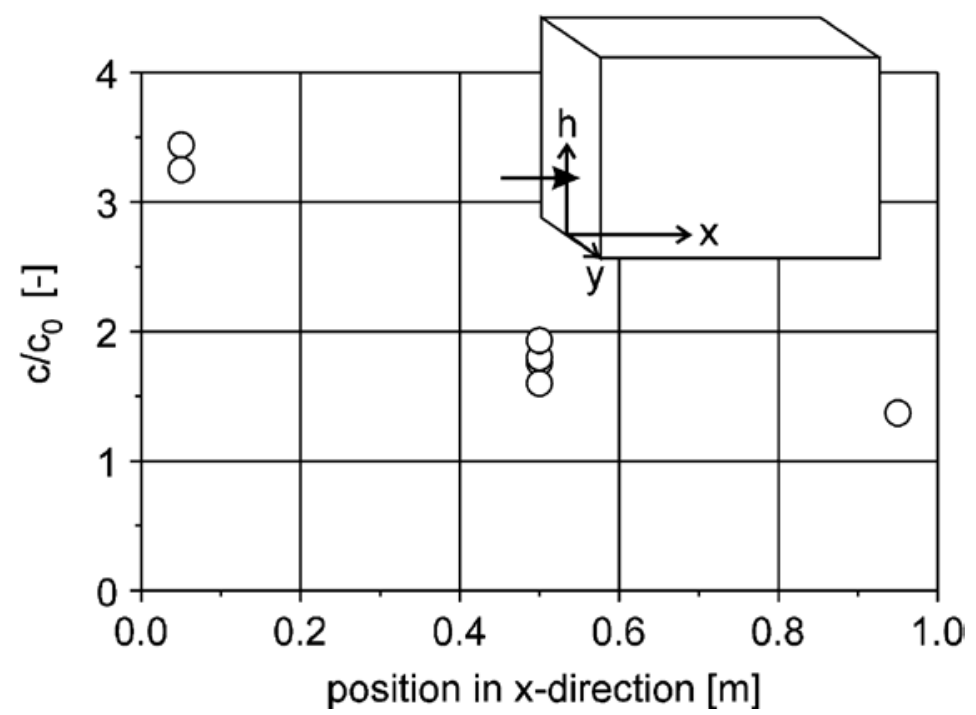

Figure3.18. Horizontal $\mathrm{CO}_{2}$ gas concentration profile at $\mathrm{h}=0.56 \mathrm{~m}$; tracer feed at $(\mathrm{x}$ $=0 \mathrm{~m}, \mathrm{y}=0 \mathrm{~m}, \mathrm{~h}=0.35 \mathrm{~m}) \mathrm{C}_{0}-$ avg. $\mathrm{CO}_{2}$, Werther et.al (2001)

The measurements depicted in figure 3.18 indicate that the normalized carbon dioxide concentration has its highest value close to the tracer feed point. With increasing distance from the feed point, the measured tracer concentration 
decreases as expected. The existence of a distinct concentration gradient shows that mixing in the horizontal direction is limited.

In figure 3.19 , the vertical profiles of the temperature differences taken at different lateral positions, $\mathrm{x}$, are plotted when the tracer was fed from the left side $(\mathrm{x}=0 \mathrm{~m}, \mathrm{y}=0 \mathrm{~m})$ at a height of $\mathrm{h}=0.35 \mathrm{~m}$. In the range $\mathrm{x}=0.25$ to $0.95 \mathrm{~m}$, the temperatures are almost independent of height at the respective locations. This indicates ideal solids mixing in the vertical direction in the bottom zone of the circulating fluidized bed. In contrast, the distinct temperature gradients in the horizontal direction show that the lateral mixing is limited, which is in agreement with previous gas concentration measurements.

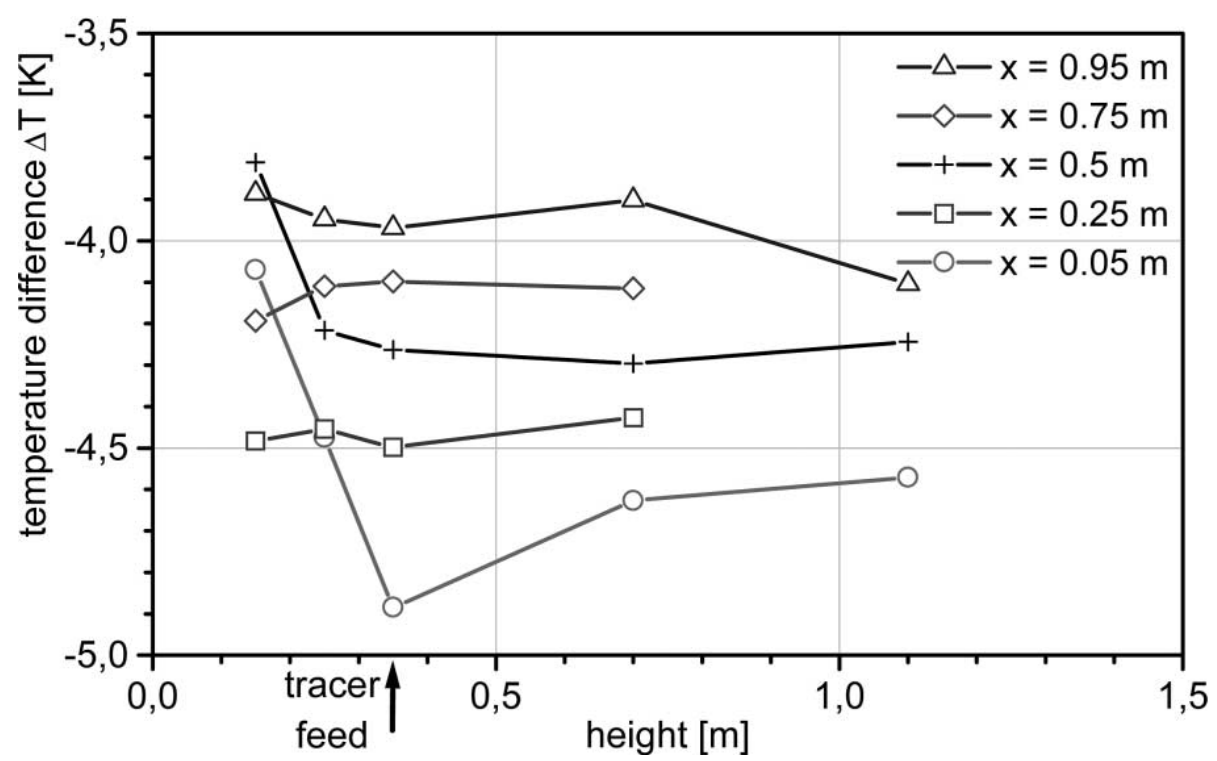

Figure3.19 Vertical profiles of local temperature differences between 10 and $110 \mathrm{~s}$ after start of feeding taken at different lateral positions $\mathrm{x}(\mathrm{y}=0$; tracer feed at $\mathrm{y}=0$, $\mathrm{x}=0.5 \mathrm{~m}, \mathrm{~h}=0.35 \mathrm{~m}$; tracer feed rate, $135 \mathrm{~kg} / \mathrm{h})$ Werther et.al (2001)

If the tracer particles are fed into the solids return line $(\mathrm{x}=1 \mathrm{~m}, \mathrm{~h}=1 \mathrm{~m}$., the temperature difference between the different lateral locations decreases further as 
shown in figure 3.20. As for the other feed locations, the temperature at each lateral location is almost independent of height. The temperature on the side which is opposite to the solids recycle entry is again slightly lower than at the locations which are closer to the solids inlet. This indicates that if the tracer particles are introduced into the solids return line, the convective transport of the solids leads to a more uniform distribution of the tracer particles in the bottom zone of the circulating fluidized bed. This effect is already practically used: in many industrial fluidized bed combustors, the fuel feed is mixed with the externally recirculated solids in order to obtain a better fuel distribution in the combustion chamber.

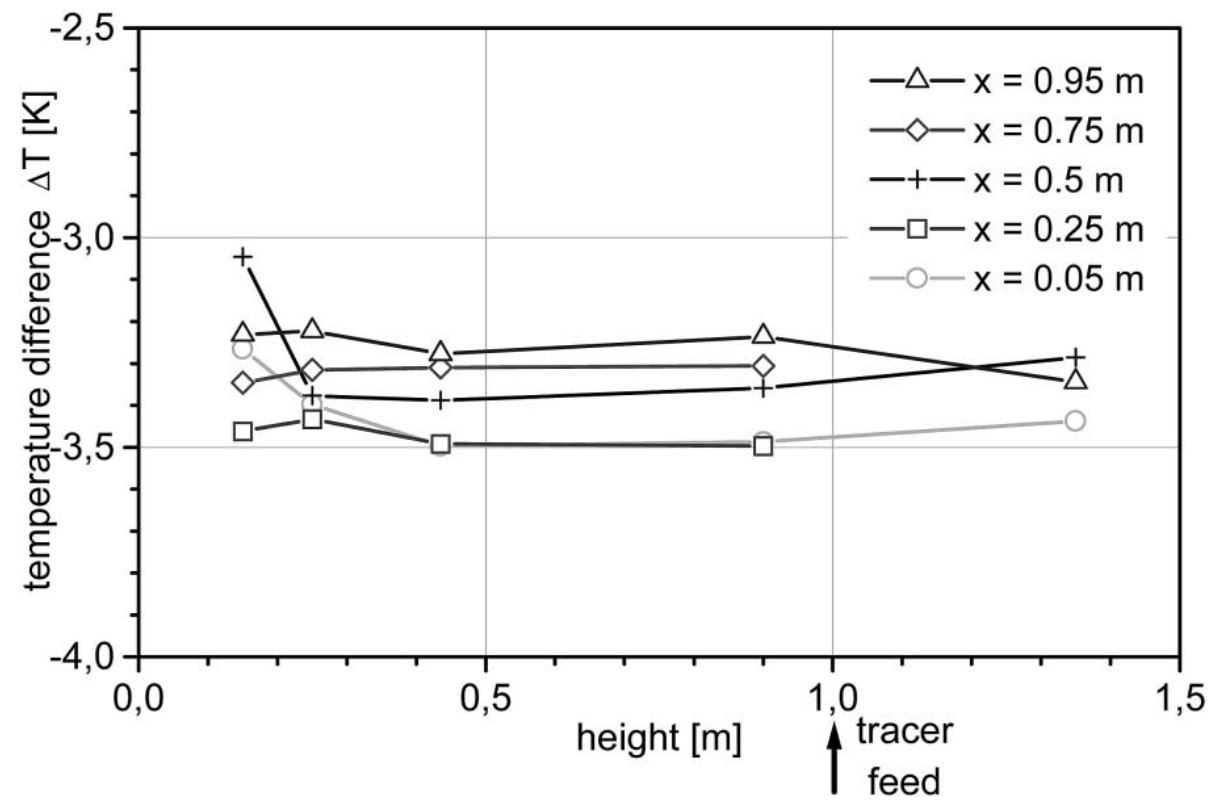

Fig3.20 Vertical profiles of local temperature differences between 10 and $110 \mathrm{~s}$ after start of feeding taken at different lateral positions $\mathrm{x}(\mathrm{y}=0$; tracer feed at $\mathrm{y}=0, \mathrm{x}=$ $1 \mathrm{~m}, \mathrm{~h}=1 \mathrm{~m}$; tracer feed rate, $135 \mathrm{~kg} / \mathrm{h})$ Werther et.al (2001)

Bi et al (1992) characterized a fast-fluidized bed. The schematic diagram of experimental apparatus used in this study is shown in figure 3.21. It 
consists of a riser, $102 \mathrm{~mm}$ in diameter and $6.32 \mathrm{~m}$ in height; a separator and secondary cyclone system, an L-valve and a large volume particle storage hopper. The particles are entrained in the upward flowing stream and exit at the top through a right-angled bend into a $102 \mathrm{~mm}$ diameter horizontal pipe connected to the separator. Subsequently, the particles are separated from the gas by the separator and secondary cyclone at the top of the riser and then fed back to the reactor by means of the non-mechanical L-valve at the bottom of the riser.

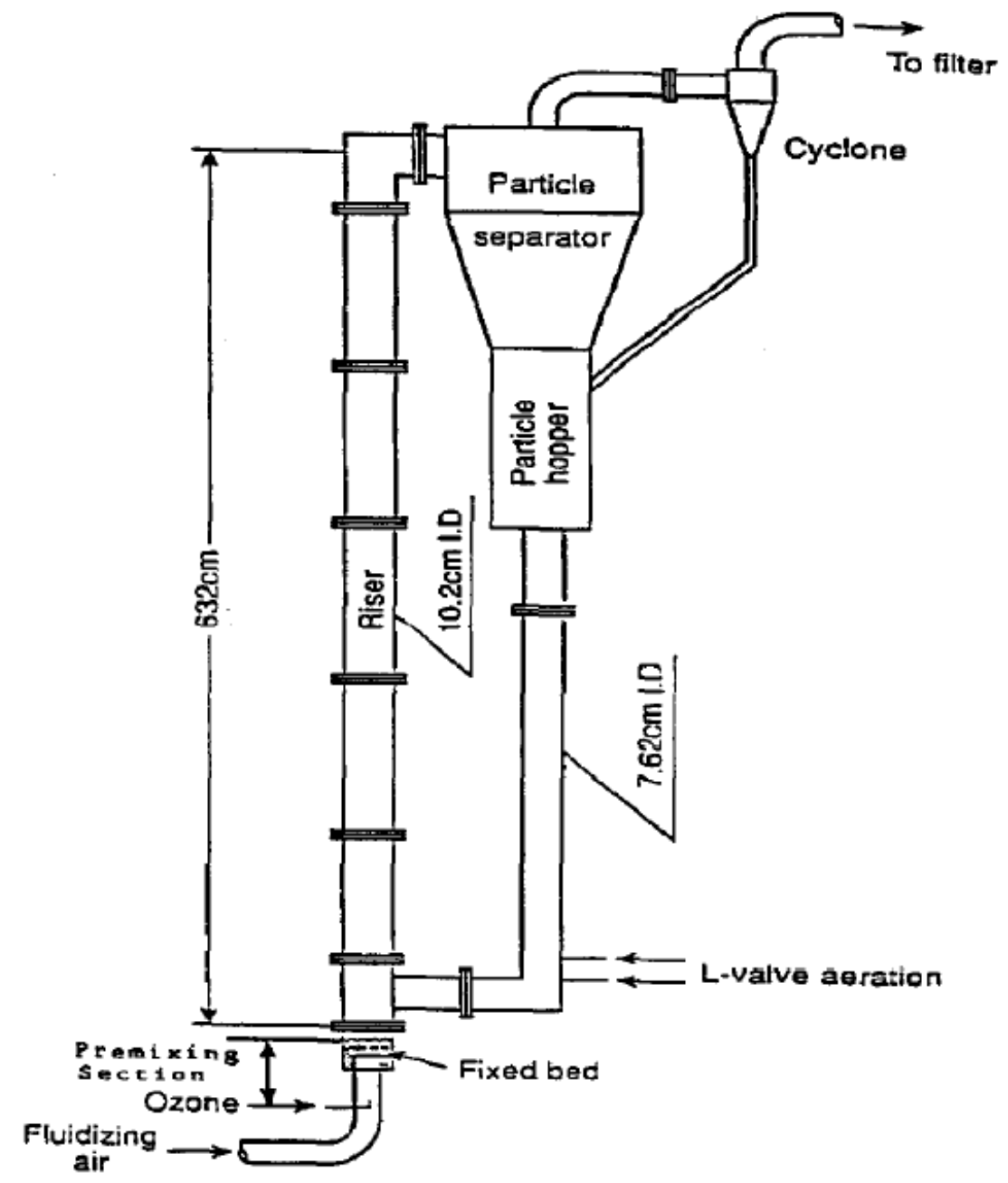

Figure3.21. Schematic diagram of the circulating fluidized bed (Bi et al 1992) 
The solids circulation rate is controlled by adjusting the flow rate of aeration air at the injecting points of the L-valve. Coarse particles placed in the system amount to $10 \%$ by volume of the total amount of particles of weight $27 \mathrm{~kg}$. The coarse particles used are $4.4 \mathrm{~mm}$ polyethylene spheres with a density of $1010 \mathrm{~kg} / \mathrm{m}^{3}$ and $2 \mathrm{~mm}$ glass beads with a density of $2500 \mathrm{~kg} / \mathrm{m}^{3}$. Ozone generated from the ozone generator is introduced into the air stream at a flow rate of $75 \mathrm{~cm}^{3} / \mathrm{s}$ by an injector located at the premixing section. To ensure that the ozone entering the column is evenly dispersed, a fixed bed with coarse particles is placed between the ozone injector and the distributor. The reaction takes place at the ambient condition $\left(23^{\circ} \mathrm{C}\right)$.

A UV detection technique reported earlier (Jiang et al., 1990a) is used to measure the ozone concentration. Figure 3.22 shows the schematic diagram of the measurement system.

To measure the ozone concentration in different axial and radial positions, an internal sampling probe is inserted through the wall of the riser. The tip of the probe is covered with a fine mesh to prevent particles from entraining into the sampling system. The sampling flow rate is very low compared to the flow rate in the bulk stream. Experiments are conducted in the absence of solids particles to evaluate a possible ozone reaction with the riser wall made of Plexiglas. The results indicate that the ozone reaction with the riser wall is negligible (Jiang et al 1990a).

Figure 3.23 shows the typical apparent bed density profiles in the riser in the presence of polyethylene (PE) coarse particles. Experimentally, it is observed that, 
at a low gas velocity and a low solids circulation rate, coarse particles are retained in the riser. This is reflected in the profiles which exhibit a dense region at the bottom section containing coarse and fine particles, and a dilute region at the upper section containing only fine particles (at $\mathrm{G}_{\mathrm{s}}=4.1 \mathrm{~kg} / \mathrm{m}^{2} \mathrm{~s}$ in figure 3.29 ). With an increase in the solids circulation rate, coarse particles are entrained by the gas and fine-particle stream and eventually carried out of the riser.

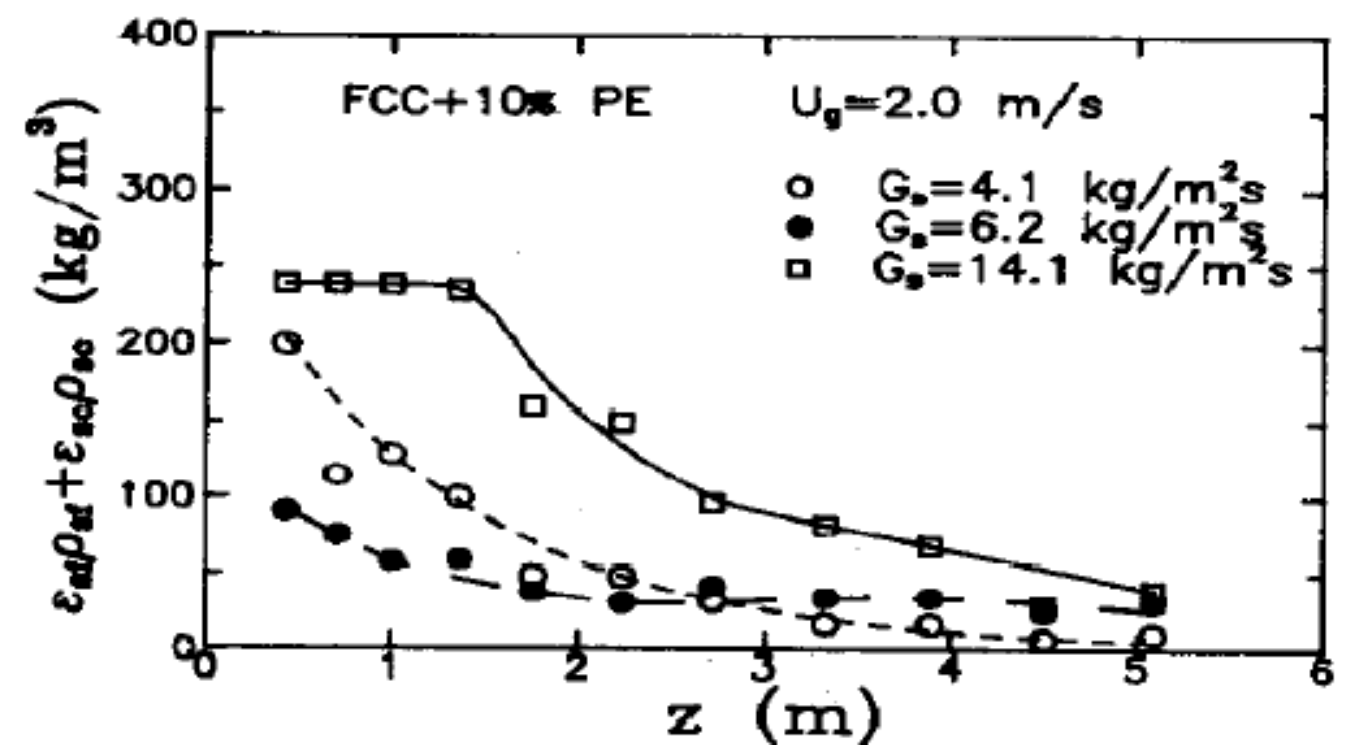

Figure3.23 Typical axial solids holdup pro6les in the riser with coarse particles (Bi et al 1992)

This, as can be seen from this figure, at $\mathrm{G}_{\mathrm{s}}=6.2 \mathrm{~kg} / \mathrm{m}^{2} \mathrm{~s}$ the variation of the apparent bed density along the bed height becomes less pronounced and the apparent bed density at the entrance region of the bed is significantly lower compared to that at $\mathrm{G}_{\mathrm{s}}=4.1 \mathrm{~kg} / \mathrm{m}^{2} \mathrm{~s}$. As the solids circulation rate is further increased, the fine particle circulation rate may reach the saturated carrying capacity and coarse-particle concentrations become relatively low. Thus, fine 
particles are accumulated at the bottom region, resulting in an S-shaped apparent bed density profile (at $\mathrm{G},=14.1 \mathrm{~kg} / \mathrm{m}^{2} \mathrm{~s}$ in figure 3.23 ).

Radial ozone concentration profiles at a vertical distance of $2 \mathrm{~m}$ from the distributor are presented in figures 3.24(a) and (b). Also shown in the figures are the corresponding apparent axial bed density profiles. It is seen from figure 3.24(a) that a parabolic profile is observed at three solids circulation rates for the system of PE coarse particles.
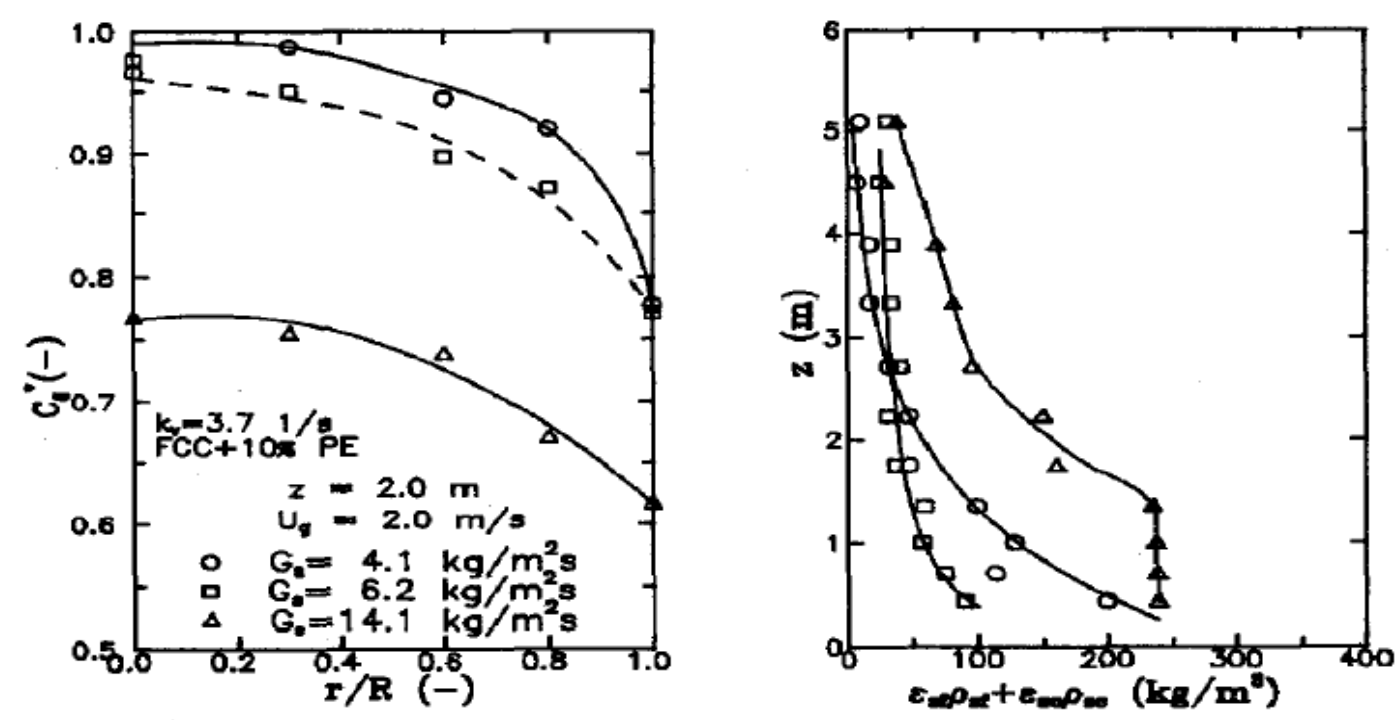

(a)
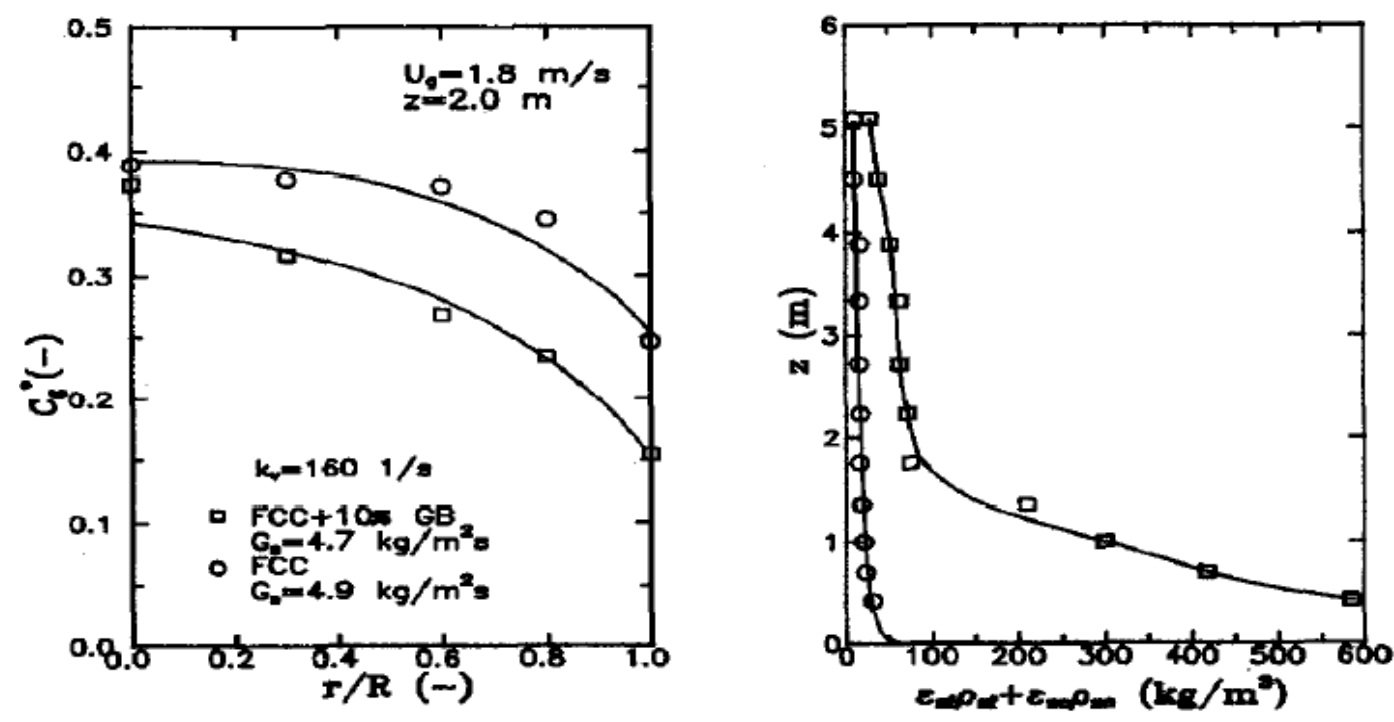

(b) 
Fig3.24 Radial distributions of ozone concentrations as a function of solids circulation rate (Bi et al 1992)

This may be in part due to higher solids holdups in the wall region as compared to those in the core region since higher solids holdups result in increased ozone conversion, and thus in a lower local ozone concentration in the wall region.

The results of figure 3.24(b) indicate that at virtually the same solids circulation rate, the riser containing the coarse GB has a radial ozone concentration profile similar to that in the absence of the coarse GB. The implication is that the radial solids holdup distribution of both systems may be similar although the solids holdup of fines appears to be higher for the system in the presence of GB, as inferred from consistently lower local ozone concentrations for that system.

Figures 3.26 to 3.28 give the results obtained by Werther et.al. In figure 3.26 radial profiles of the tracer gas concentration were measured in a plane downstream of the injection level. Injection into the empty tube is compared with injection under circulating fluidized bed conditions. The empty tube measurements show that the tracer gas has already penetrated about $0.1 \mathrm{~m}$ into the radial direction and no tracer is observed at the vessel center. A significant change in the concentration profile occurs after switching to the circulating fluidized bed operation. The profile is now flattened and a significant amount of the tracer gas is detected on the center line of the column.

One might conclude from this comparison that the presence of the solids simply leads to an enhancement of the radial gas dispersion. 
That this explanation would be too simple becomes obvious from the measurements shown in Figure 3.27 where two gas concentration profiles are compared which have been measured downstream and upstream, respectively of the injection plane. The backmixing effect is marked although the distance between the measuring plane and injection level is greater for the upstream measurements the concentrations measured in the vicinity of the wall in this case are still much larger than in the case of the downstream measurements.

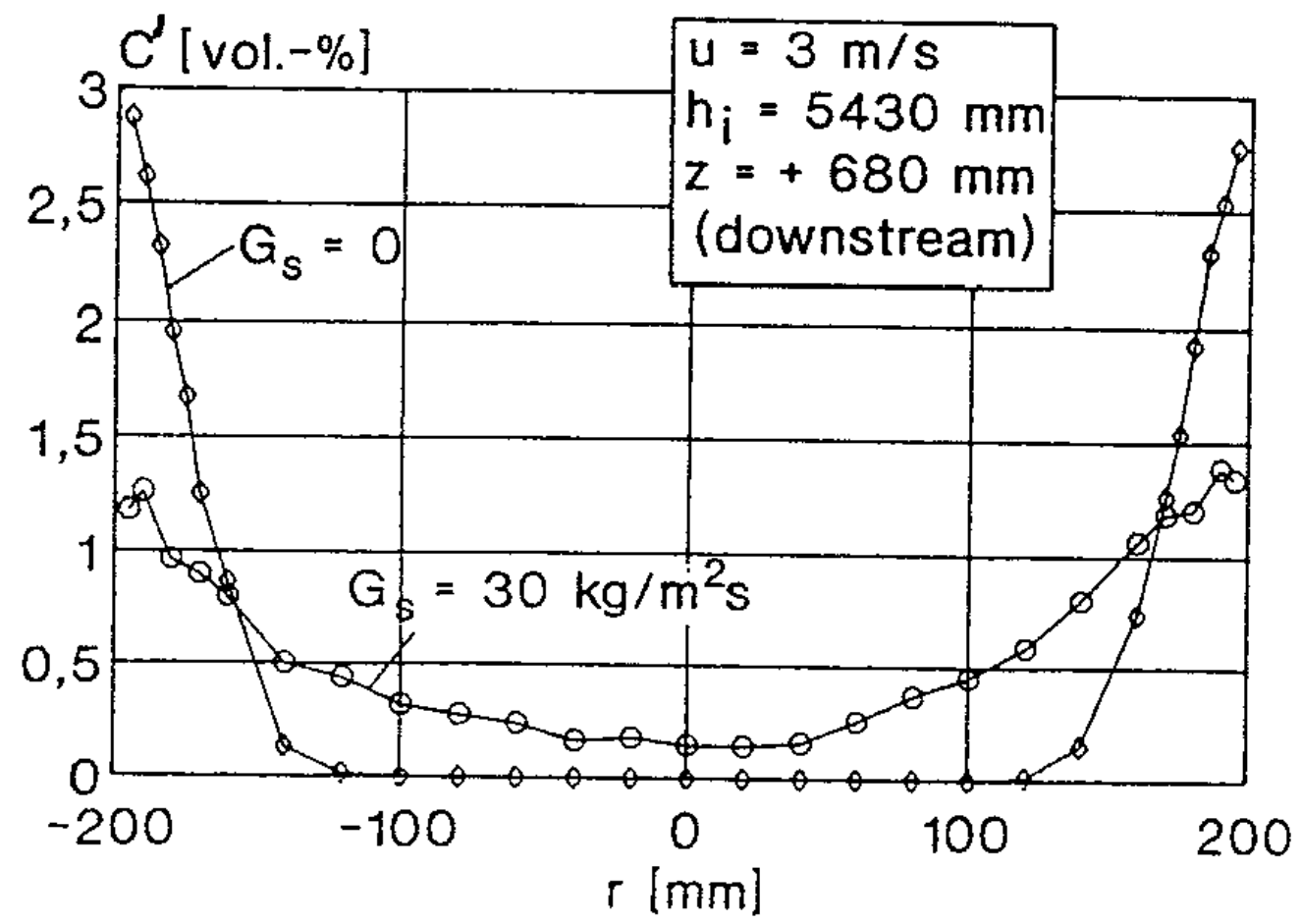

Figure3.26. Radial Profiles of Tracer Gas Measurements (Werther et al 1999) 


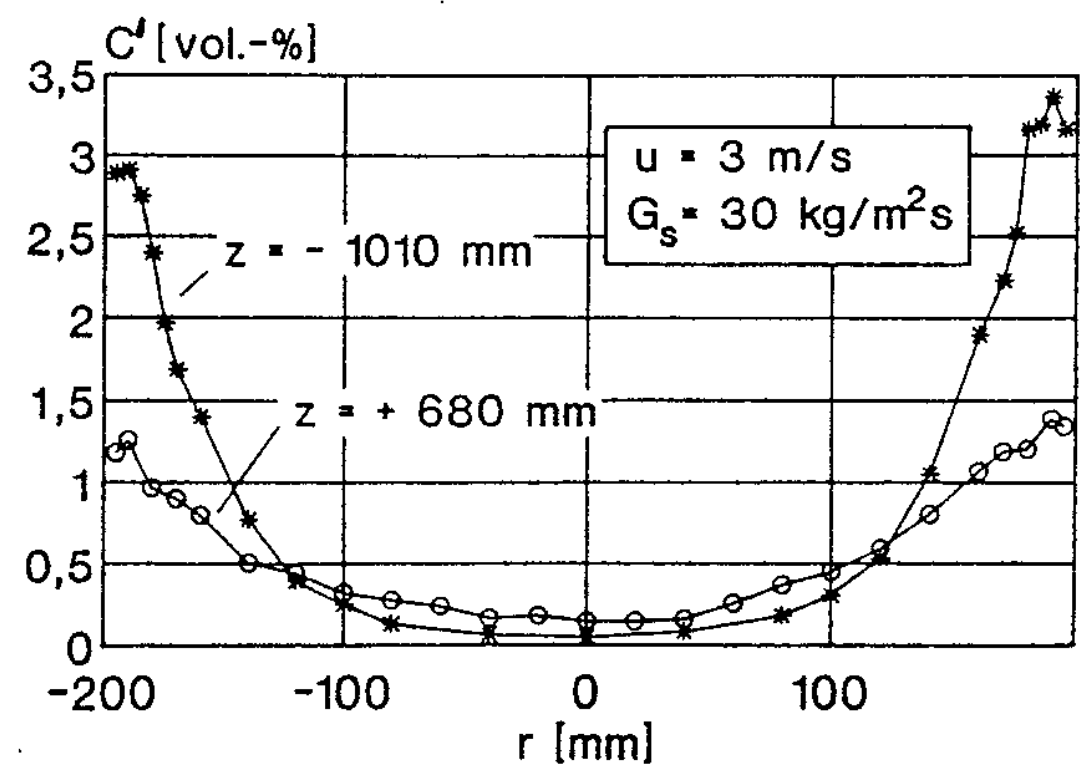

Figure3.27. Upstream and downstream tracer profiles (Werther et al 1999)

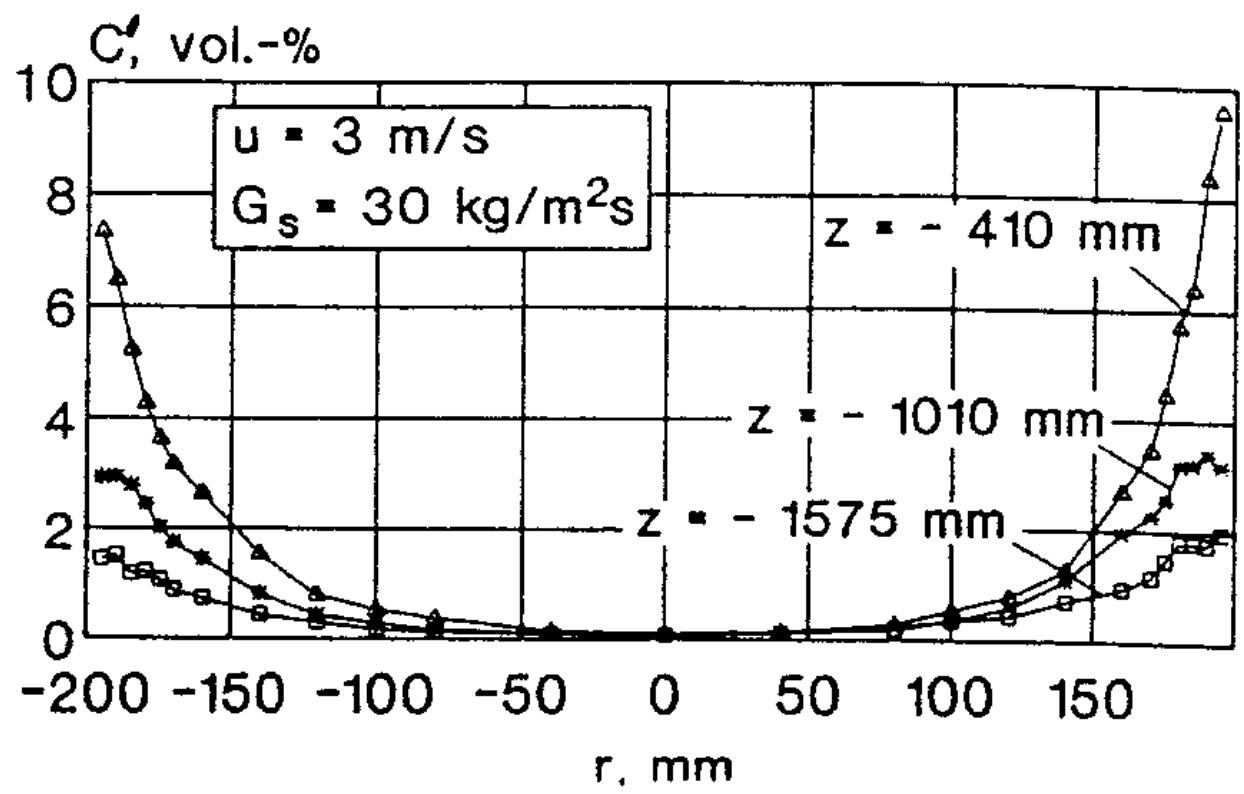

Figure3.28. Stability of Down flowing tracer gas (Werther et al 1999)

The extent of this effect can only be accounted for in a model which considers a down-flow of the gas near the wall. Figure 3.28 shows that this down-flowing current is quite stable over large distances. 


\section{Chapter IV}

\section{Tracer Gas Study Results}

\subsection{Introduction}

In this chapter, the experimental results obtained from pressure measurements and tracer gas experiments are discussed. The chapter starts with the presentation of the results of the static pressure measurements and continues with the results of the gas mixing experiments. The radial gas mixing and the axial gas mixing are discussed separately.

\subsubsection{Different Regimes and Average Bed Voidage}

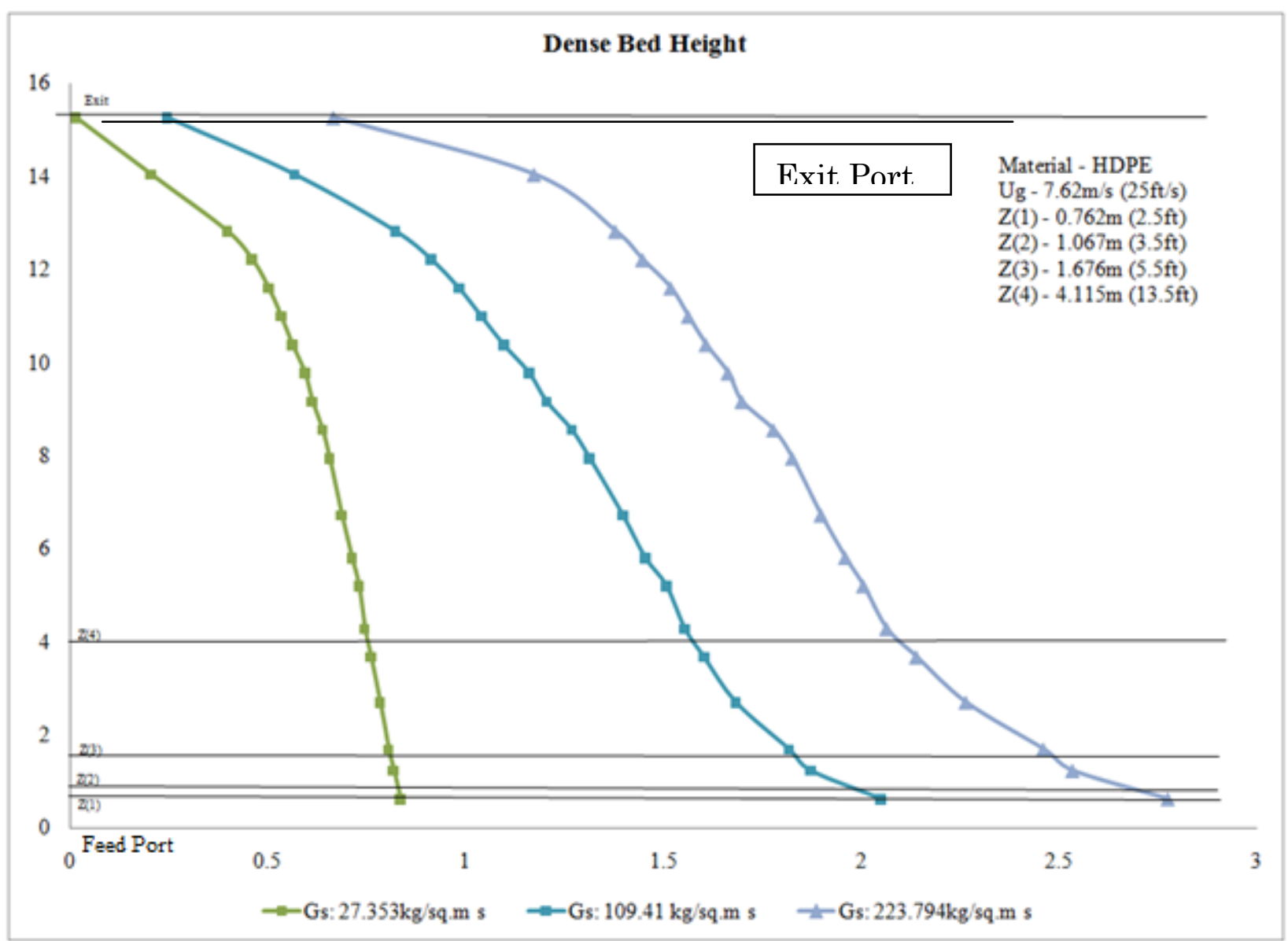

Fig4.1 Dense Bed Height for the test matrix conditions with respect to riser height

Figure 4.1 gives the dense bed height for the three levels of solids circulation rates of the test matrix. There is no dense bed formed for the low solids circulation rate however there is dense bed formed for the intermediate and high solids circulation rates. The dense bed height 
was found to be between 1 to $2 \mathrm{~m}$ for both the cases with respect to the center line of the LValve.

\subsubsection{Fluidization Regimes for the Test Matrix Operating Conditions}

This section is aimed at identifying quantitatively the regimes of fluidization in the range existing in the envelope of the operating conditions selected for this work. To determine the regimes an amplitude analysis, yielding the average absolute deviations (AAD) i.e. a measure of the average amplitude is used. The AAD is a robust invariant which quantifies the average amplitude of the time series.

$$
A A D=\frac{1}{N} \sum_{i=1}^{N}\left|x_{i}-\bar{x}\right|
$$

Where $\bar{x}$ is the mean of the time series (pressure fluctuations) and $\mathrm{N}$ is the number of samples in the time series. The AAD is comparable to the standard deviation and shows similar dependence on operating conditions. Figure 4.5 illustrates the work done by Zijerveld et.al. (1998) where below $\mathrm{U}_{\mathrm{c}}=2.2 \mathrm{~m} / \mathrm{s}$ the bed is in the bubbling or slugging regime and the AAD increases for increasing superficial gas velocity. $U_{c}$ in this study represents the transition velocity, slugging bed to intermediate turbulent bed $(\mathrm{m} / \mathrm{s})$. The AAD decreases beyond $\mathrm{U}_{\mathrm{c}}$ because the slugs are broken up. The AAD of the series of runs with a settled bed height of $0.56 \mathrm{~m}$ levels off at high superficial gas velocity when a bottom bed no longer exists. Whereas the AAD of the series of runs with a settled bed height of $0.85 \mathrm{~m}$ levels off at high superficial gas velocity when there is already considerable solids mass flux $\left(\mathrm{G}_{\mathrm{s}}=42 \mathrm{~kg} /\left(\mathrm{m}^{2} \mathrm{~s}\right)\right.$ at $\left.4.5 \mathrm{~m} / \mathrm{s}\right)$.

Zijerveld et.al, found that as the superficial gas velocity increased the bed from a bubbling bed(2) - slugging bed (5) - intermediate turbulent/turbulent bed (6) - circulating slugging bed (8) - dilute transport flow (9). Figure 4.6 gives the AAD analysis depicting the change in fluidization regimes for test matrix in this study. 

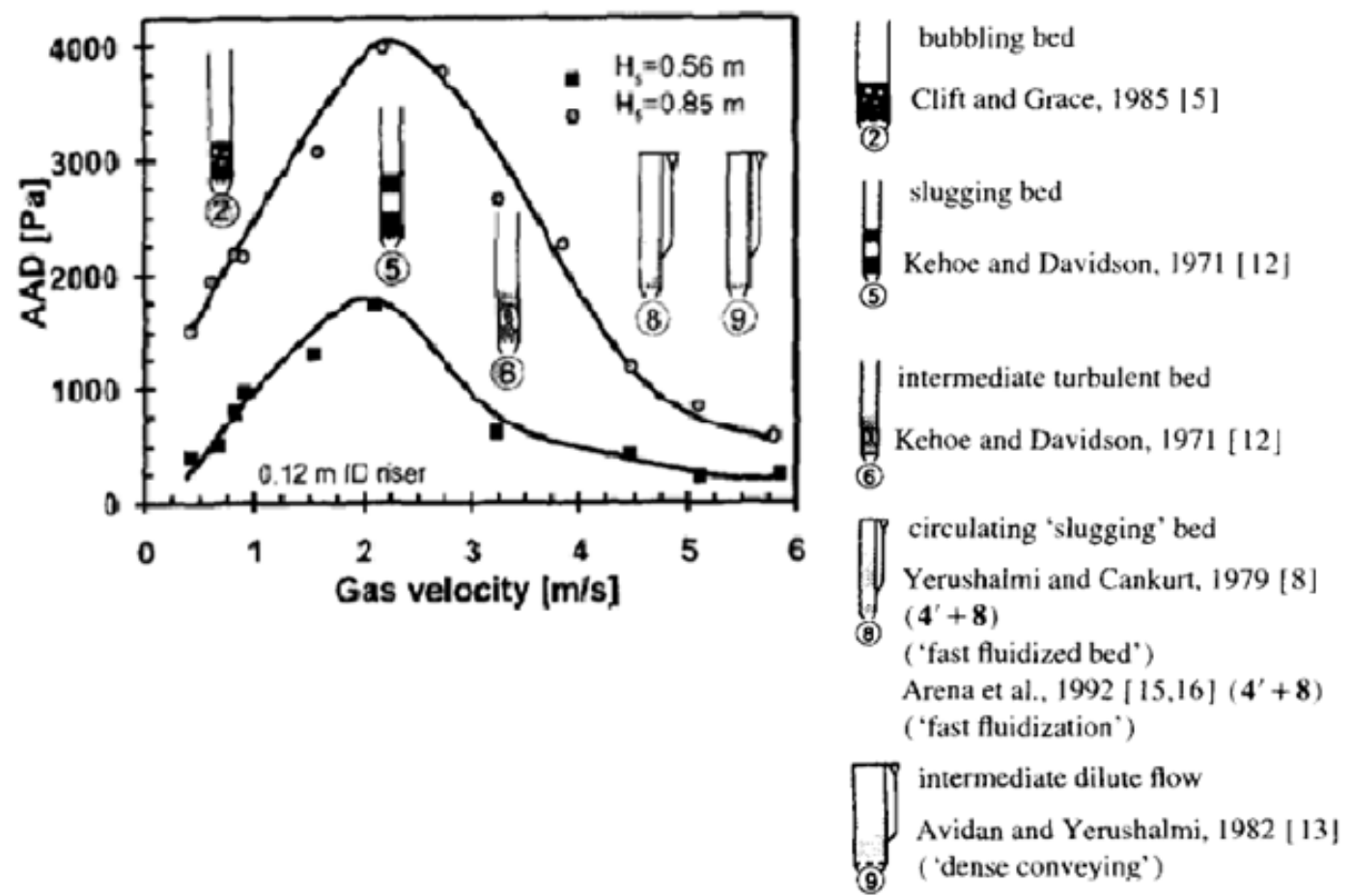

Fig 4.5. AAD as a function of superficial gas velocity for route 1 in the $0.12 \mathrm{~m}$ id riser; the pressure fluctuation probe is at $0.57 \mathrm{~m}$ (Zijerveld et al 1998) 


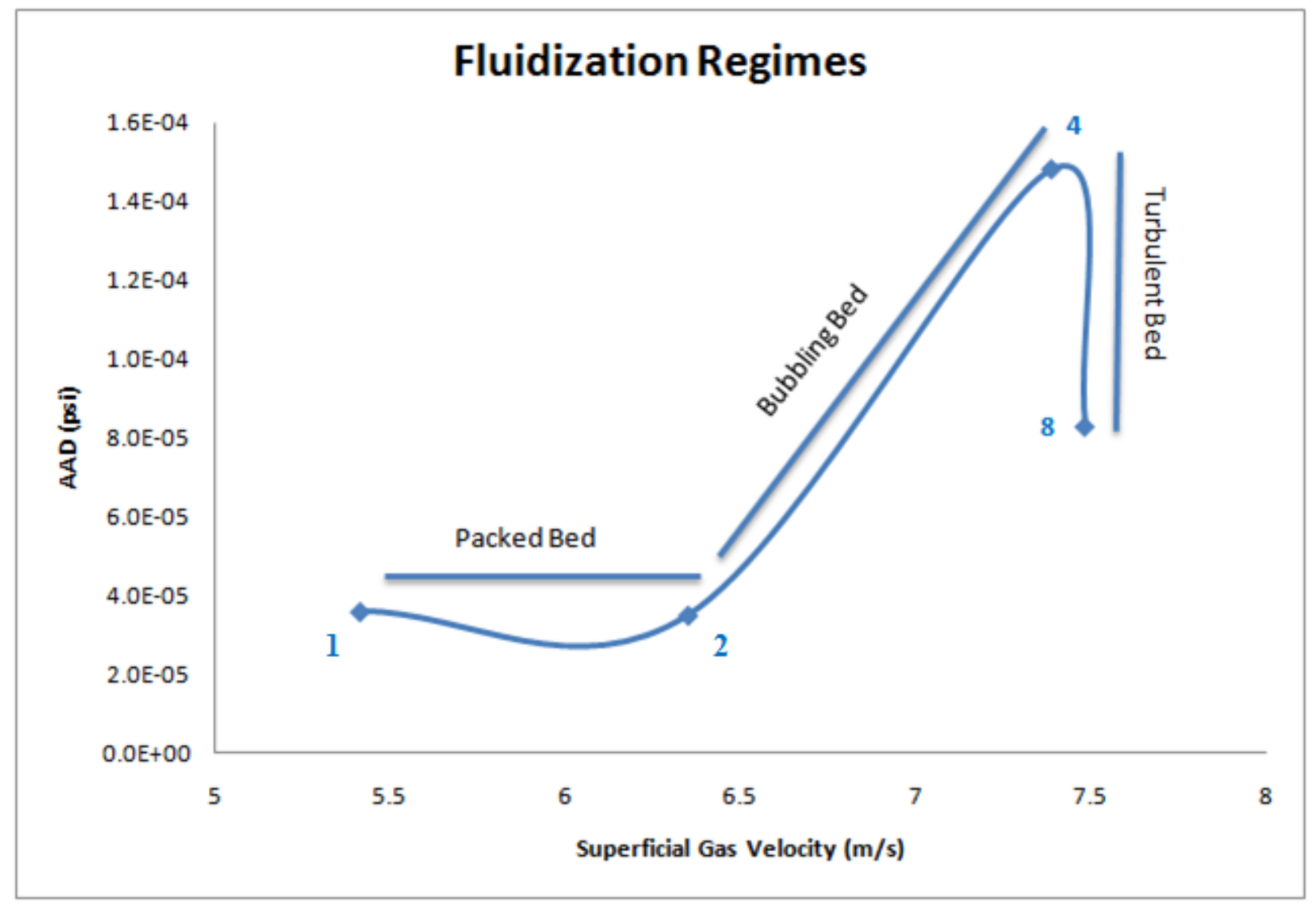

Fig4.6 AAD as a function of superficial gas velocity for the factorial test matrix

Figure 4.6 is quite similar to the result of Zijerveld et.al. Hence as the superficial gas velocity increases the dense bed transitions from a bubbling bed - to a slugging bed to a turbulent bed. The AAD calculations for this work was based on the high speed pressure measurements made at $0.762 \mathrm{~m}(2.5 \mathrm{ft})$.

\subsection{Co Flow Hypothesis}

In chapter 1 , section 1.5 , it was mentioned that this tracer gas technique is being used to determine the distribution of a stream of solids being fed into the riser and the concentration of the fed solids in the dense entry regions. This essence of this technique is represented in figure 4.1. The tracer gas is injected in the middle of the solids i.e., the point of the injection is in the middle of the angle of repose. The trapped tracer gas flows along with the solids into the riser. The tracer gas essentially flows along with the solids in the riser till the time the stream of solids disintegrates. At this point, the stream of the solids has been dispersed over a local volume in the riser. 


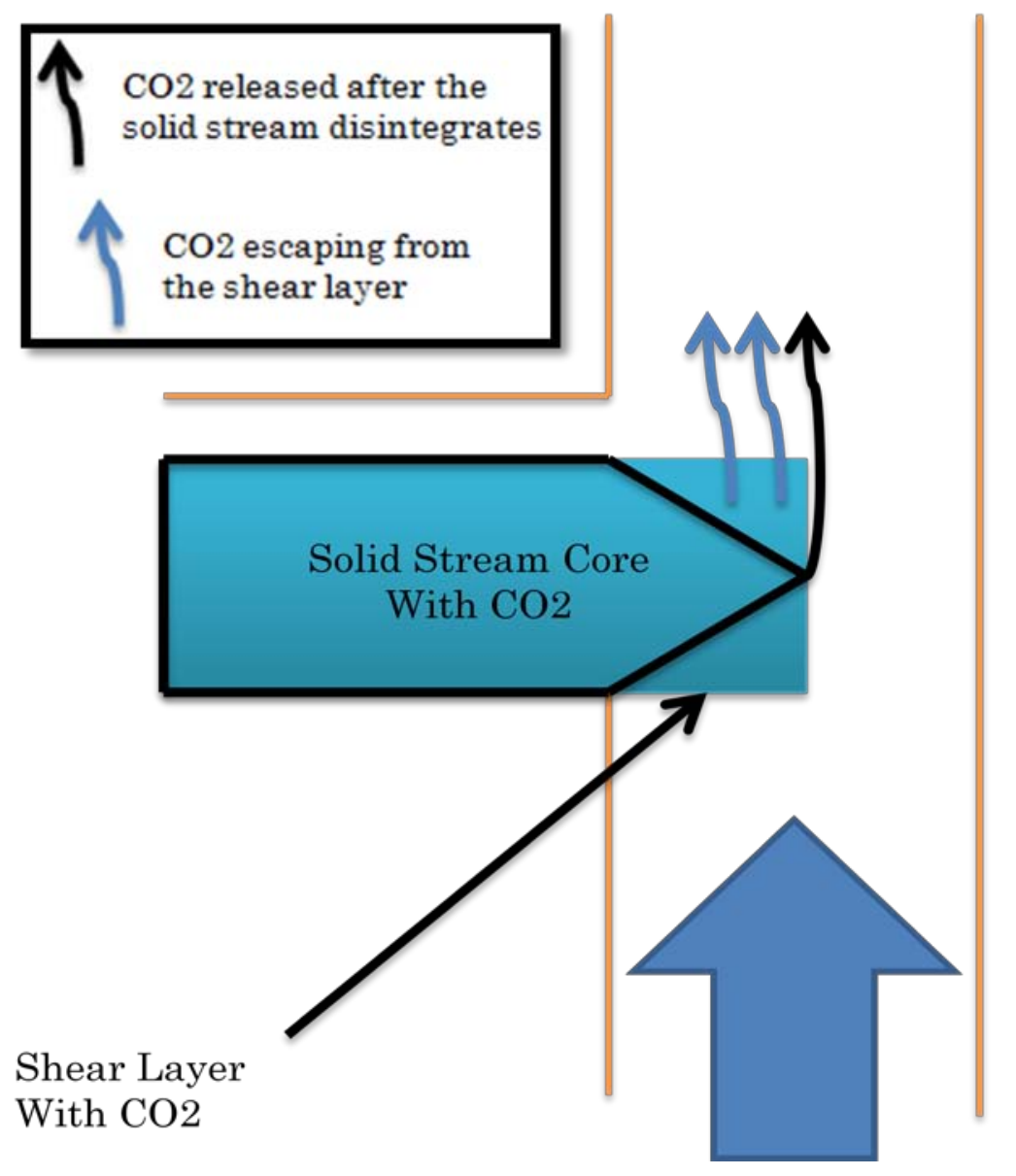

Fig4.7 Co Flow of $\mathrm{CO}_{2}$ tracer gas and Solids

This co-flow condition of the solids and gas will eventually separate and will not hold true for all the dense entry region of the riser. As a result this technique can be used to determine the solids concentration of the injected particles in the lower sections of the bottom dense region of the riser. As such in the lower sections of the dense entry region, a high tracer gas concentration should not just indicate a higher concentration of the gas but also a higher concentration of the solids flowing in to the riser.

It has been observed based on the experience in drying, that it is difficult to remove a gas from a flowing stream of solids. Based on this observation if the tracer gas is injected in the middle of the flowing stream of solids being fed into the riser, the gas will essentially remain in the stream until the stream disintegrates. The gas does not diffuse out from the solids stream quickly. Thus a co-flow condition develops where the tracer gas is entrained in the injected stream of solids and moves with the solid stream till the time the solids stream disintegrates. This co flow condition is shown in figure 4.7. 
Depending on the combined momentum of the stream of solids being fed into the riser from the L-Valve and the dense bed solids above the distributor, three distinct $\mathrm{CO}_{2}$ tracer gas profiles can be visualized. The solids coming from the L-Valve can have a low magnitude of momentum, an intermediate magnitude of momentum and a high magnitude of momentum. The expected tracer gas profiles for these three different magnitudes of momentum are shown in figures 4.8, 4.9 and 4.10 respectively. As the tracer gas flows up the riser, the sampling probes, sample the air from the riser from 12 radial positions and 4 axial positions. The 12 radial and 4 axial positions are shown in figure 4.11. At the lower levels of the dense entry region, where it can be assumed that the co-flow condition and that the solids stream has not disintegrated, the tracer concentration profiles can be used as an indicator for the distribution of the solids fed into the riser.
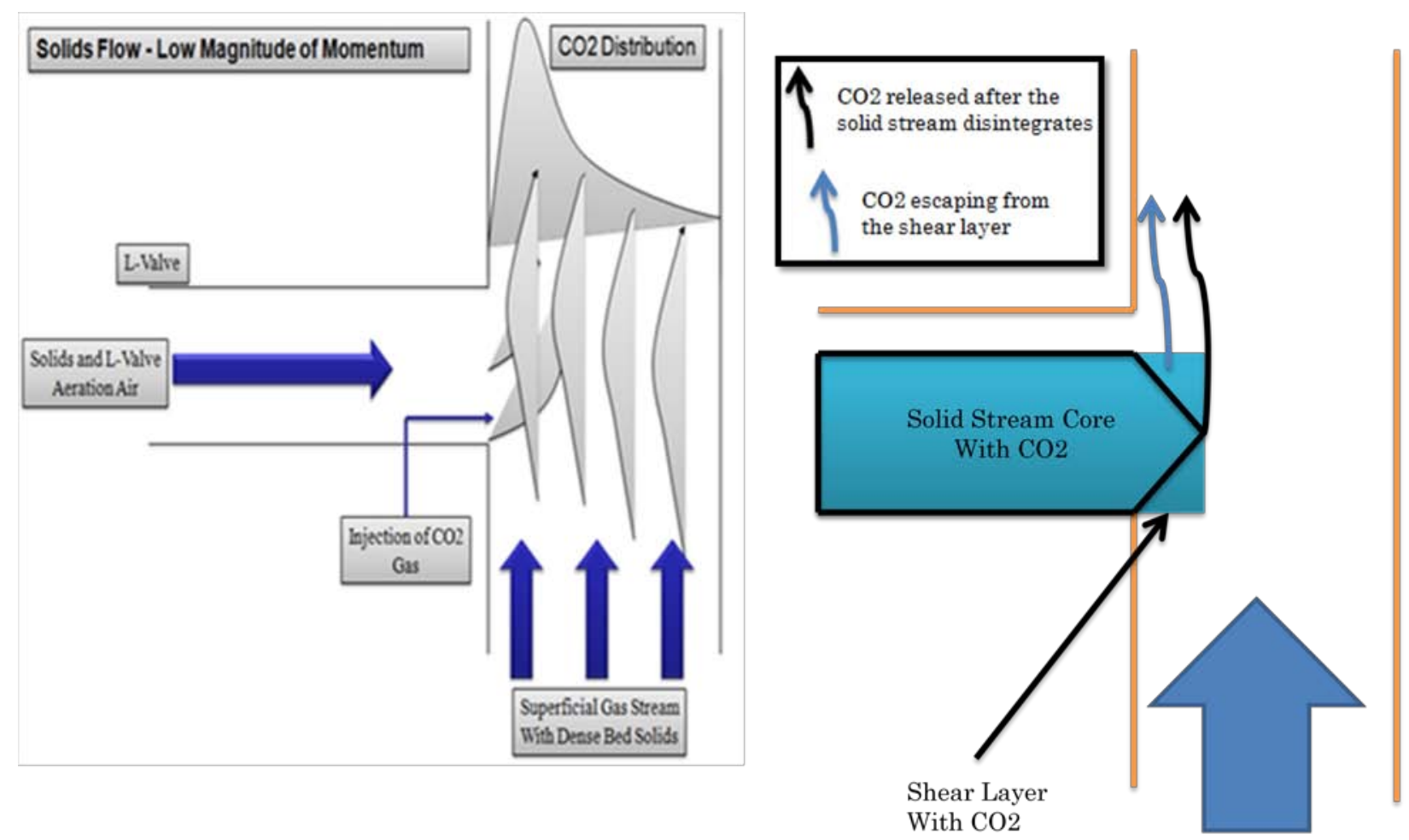

Fig4.8 Expected tracer profile for low solids momentum 


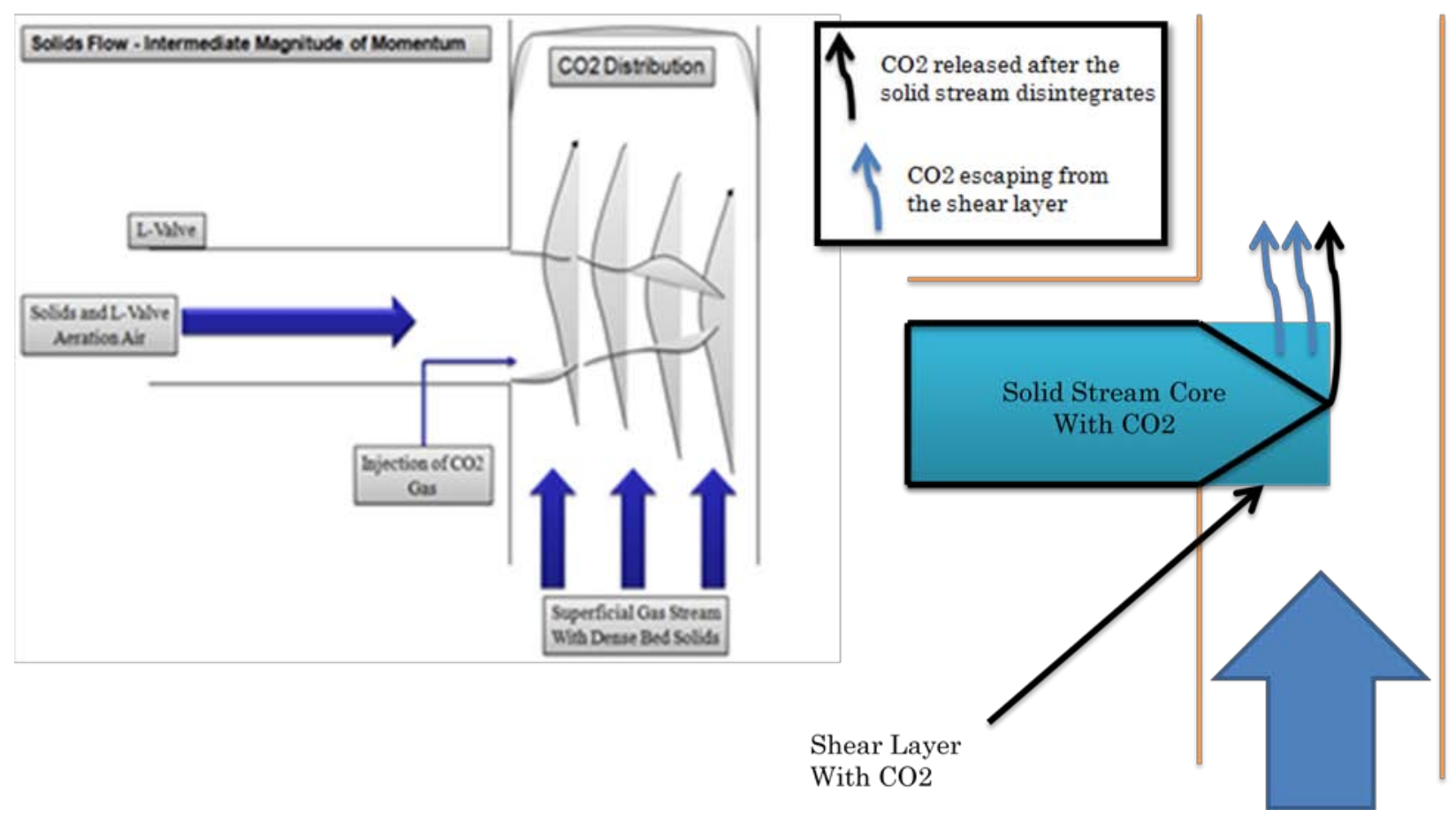

Fig4.9 Expected tracer profile for intermediate solids momentum

However at higher elevations, it is highly possible that the solids stream has disintegrated and so the tracer gas profiles can only be used as a measure to gauge the gas mixing process.
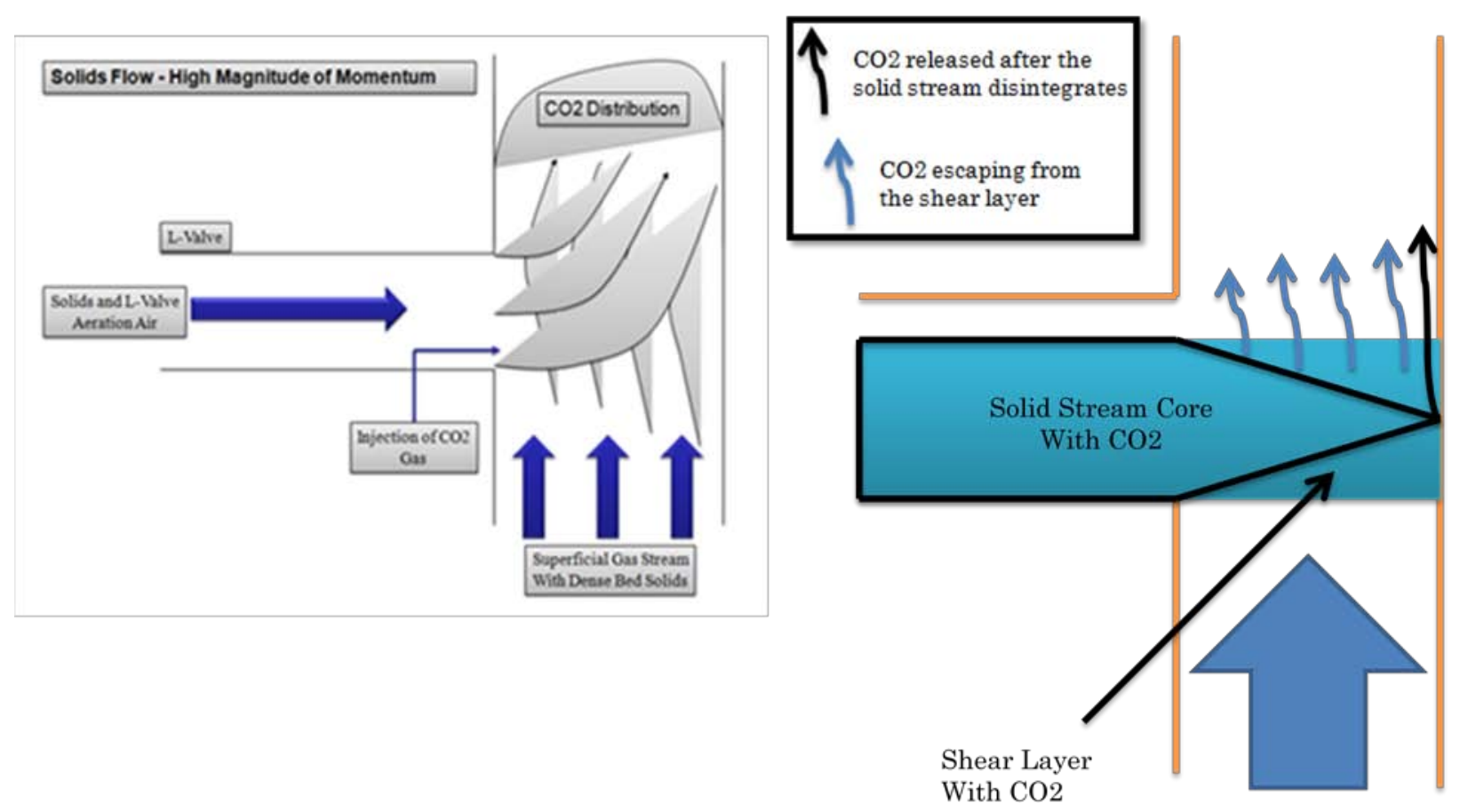

Fig4.10 Expected tracer gas profile for high momentum of solids 
A detailed explanation or the reason behind the three distinct profiles will be given in the results and analysis section.

The axial positions were $0.762 \mathrm{~m}(2.5 \mathrm{ft}), 1.067 \mathrm{~m}(3.5 \mathrm{ft}), 1.676 \mathrm{~m}(5.5 \mathrm{ft})$ and $4.115 \mathrm{~m}(13.5 \mathrm{ft})$. Two factors led to the selection of these axial positions

1. The primary focus of this work is on the dense entry region of the riser

2. On the available ports for measurement purposes. As most of the ports were being used to measure the pressure drop and other parameters across the Riser the selection of the ports was quite constrained.

\subsection{Tracer Gas Measurements}

Figures 4.11 shows the locations at which the tracer gas measurements were made. Figure 4.12 shows the schematic layout of the probe and detectors in the dense entry region of the riser.

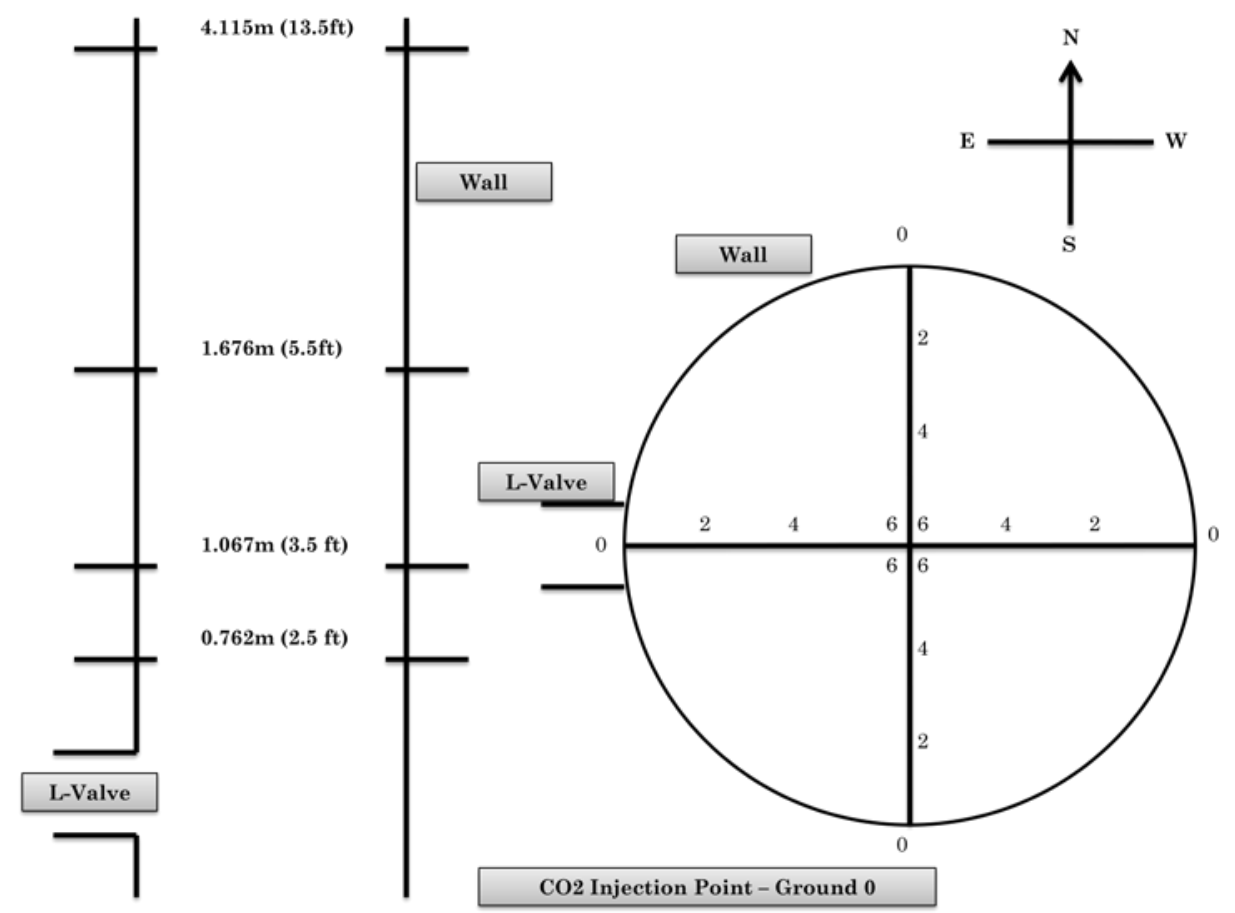

Fig4.11 Locations at which tracer gas measurements were taken in the dense entry region

The resulting tracer gas concentration curves represent how the tracer gas is dispersed in the radial direction at various axial elevations in both azimuthal positions. The location of the injection and the sampling probes along the riser height with respect to axial solids distribution is important since the resulting gas dispersion will depend on the gas-solid regime between the two 
probes. For instance, if the probes are located in the fully developed region where the axial solids distribution is relatively constant the information will be specific to that dilute region.

There are several pitfalls that have to be avoided in radial mixing experiments (Grace et al 2009); the tracer gas injection and gas sampling velocities may affect the gas dispersion. For tracer gas injection, the tracer gas velocity at the tip of the injection probe should be less than the local gas velocity since injection at a velocity greater than the maximum gas velocity at the centerline can produce jet formation which in turn increases gas dispersion. In this study, the injection probe tip diameter was chosen as $9.525 \mathrm{~mm}$ (3/8in). This ensures a tracer gas velocity which is less than the local gas velocity for all the tracer gas flow rates and superficial gas velocities used during the experiments.

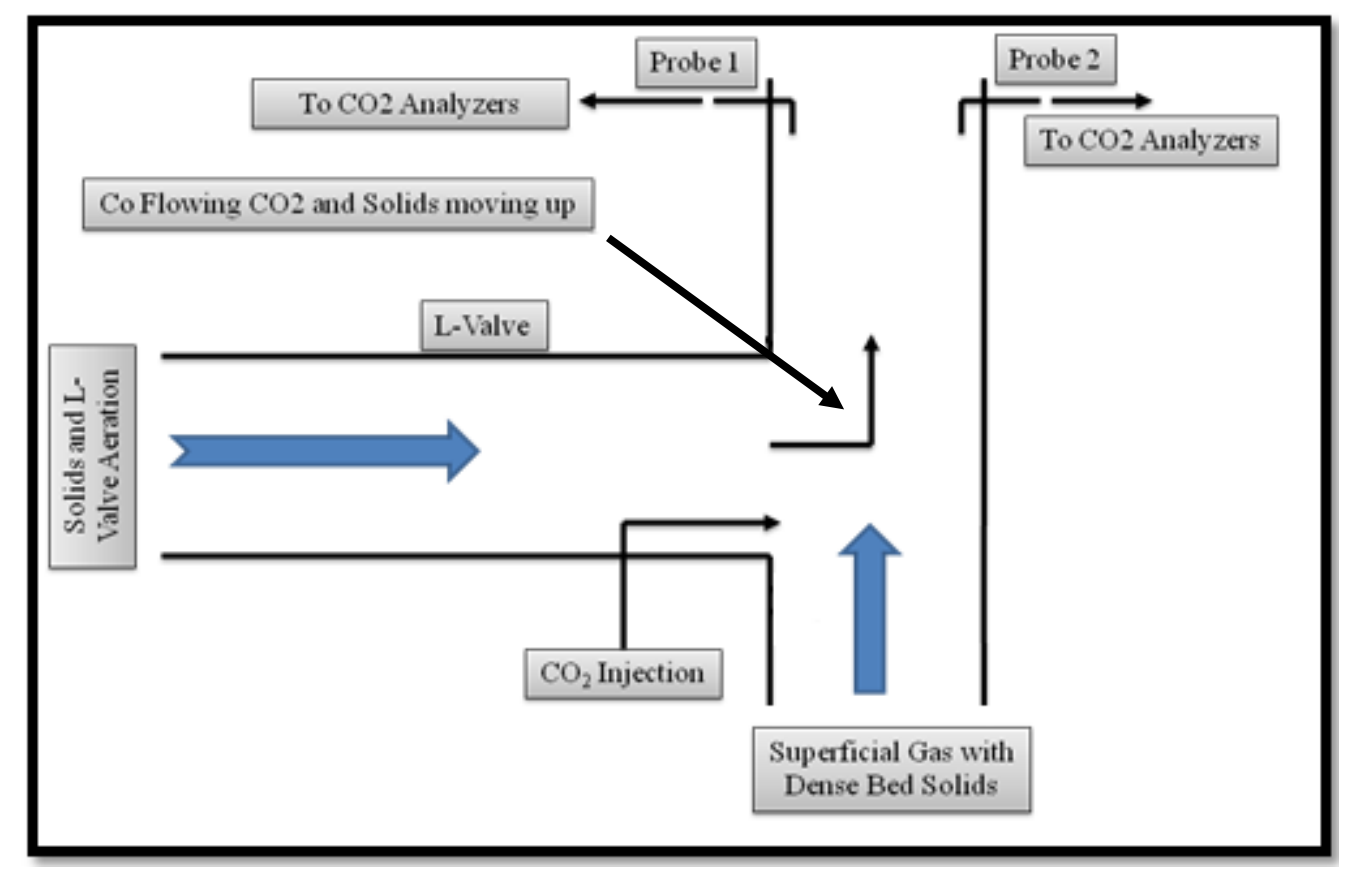

Fig 4.12 Schematic of the probe and injection layout

For instance, for a typical tracer gas flow-rate of $0.566 \mathrm{~m}^{3} / \mathrm{hr}$ at a superficial gas velocity of $5-8$ $\mathrm{m} / \mathrm{s}$, the tracer injection velocity becomes $0.781 \mathrm{~m} / \mathrm{s}$ which is well below the average gas velocity in the riser. Furthermore, a porous stainless steel filter was groove fitted to the tip of the injection probe so that the tracer gas has a uniform entrance to the riser without a jet formation.

For gas sampling, in their experiments, Amos et al. (1993) did not see any effect of the sampling gas velocity on the radial dispersion when it was kept smaller than the local gas velocity. The sampling flow rate in this study was determined by the limitations of the gas analyzer; the specifications dictated it to be in the range of $1.1-2.0 \mathrm{l} / \mathrm{min}$ and it was set to be 
between $1.6-2.0 \mathrm{l} / \mathrm{min}$ during the experiments. With a sampling probe of $9.525 \mathrm{~mm}$ ID, the suction velocity at the tip varied between 1.35 and $1.7 \mathrm{~m} / \mathrm{s}$ with varying suction flow rate. This is a fairly low velocity compared to the superficial gas velocities $(5-8 \mathrm{~m} / \mathrm{s}$ ) used in this study. Preliminary tests showed no difference in tracer gas concentration as the suction flow rate changed between $1.6-2.0 \mathrm{l} / \mathrm{min}$

\subsection{Radial Tracer Gas Dispersion Profiles}

\subsubsection{Tracer Gas Profile Results}

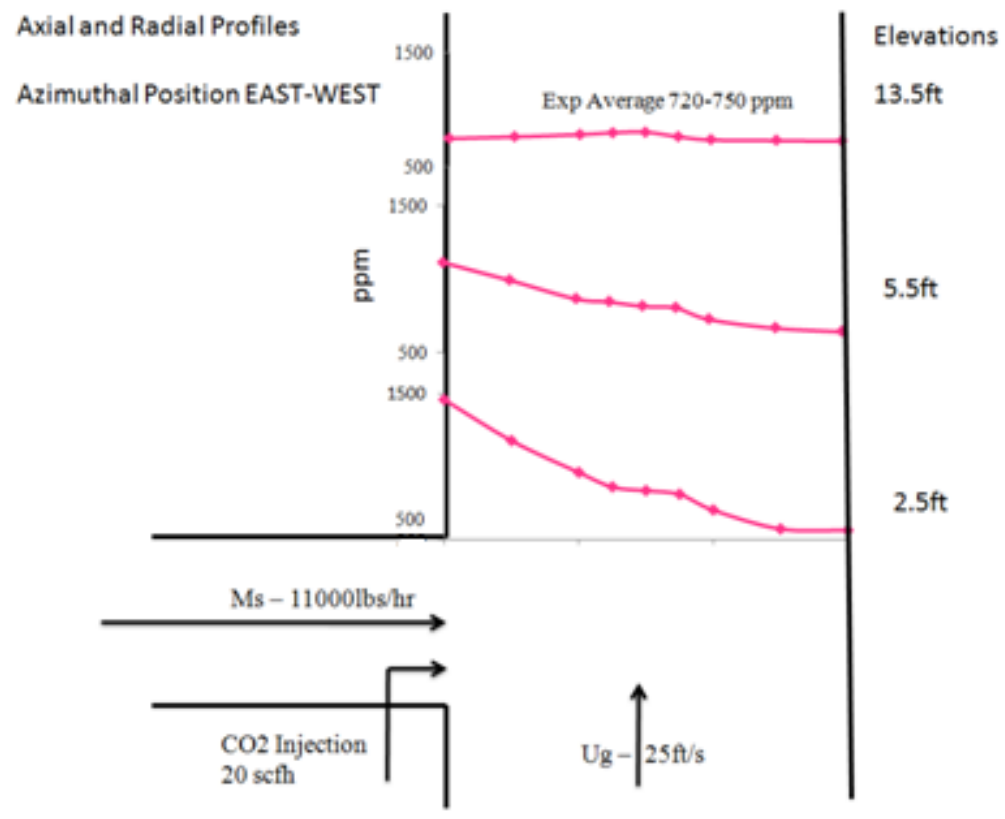

Fig 4.13 Radial dispersion of the tracer gas in the east west direction for low solids feed 


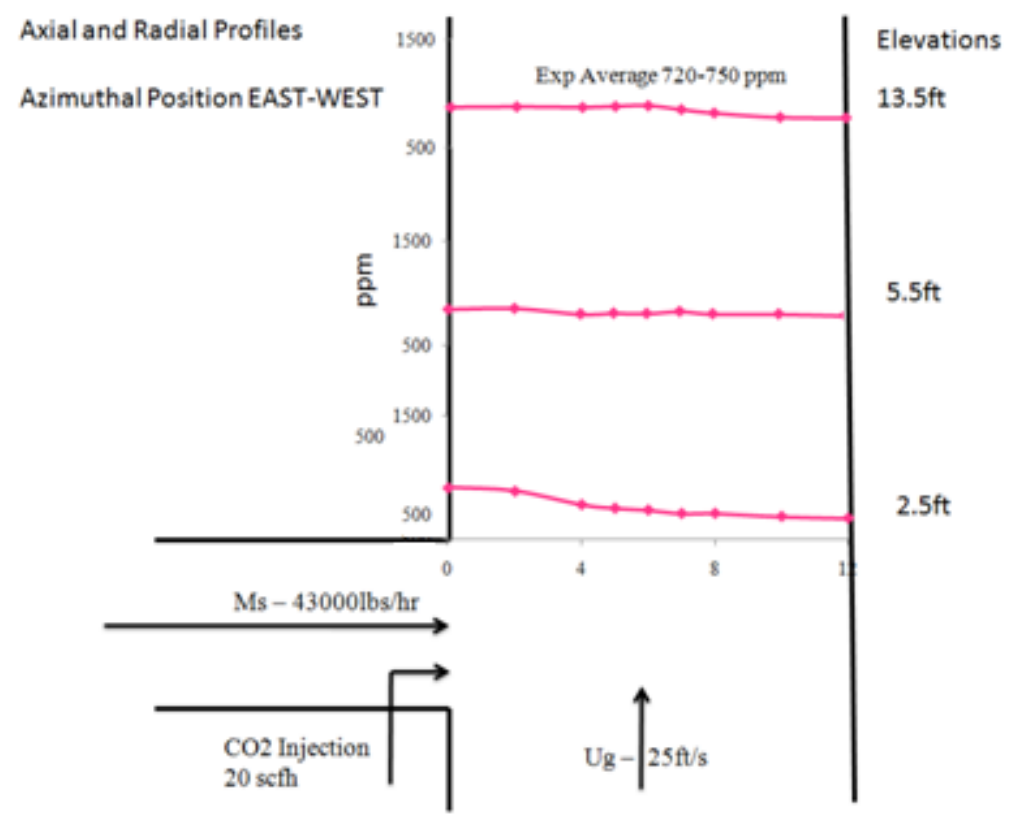

Fig 4.14 Radial dispersion of the tracer gas in the east west direction for intermediate solids feed

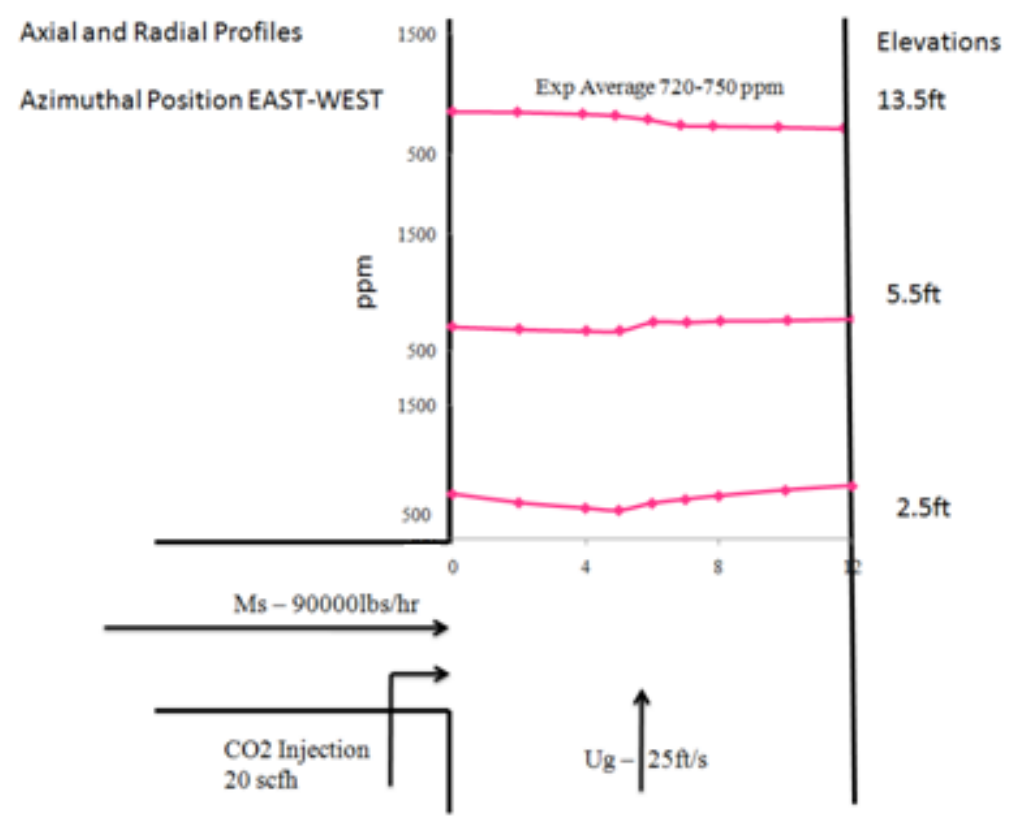

Fig 4.15 Radial dispersion of the tracer gas in the east west direction for high solids feed 


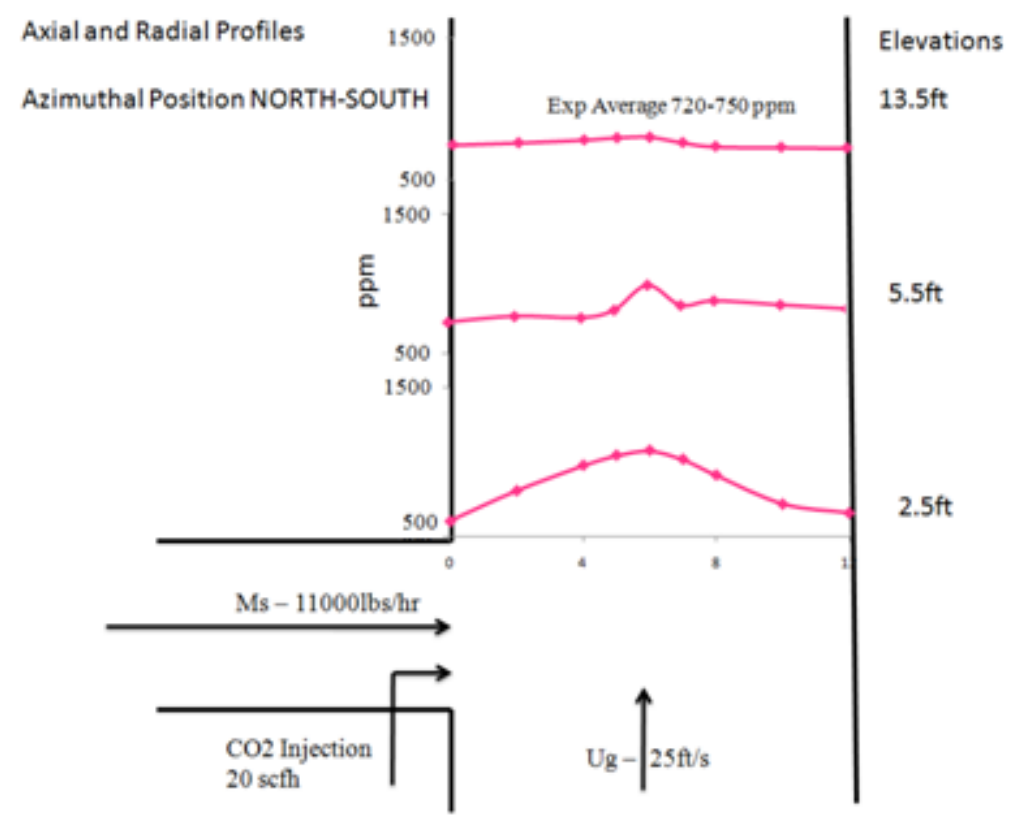

Fig 4.15 Radial dispersion of the tracer gas in the north south direction for low solids feed

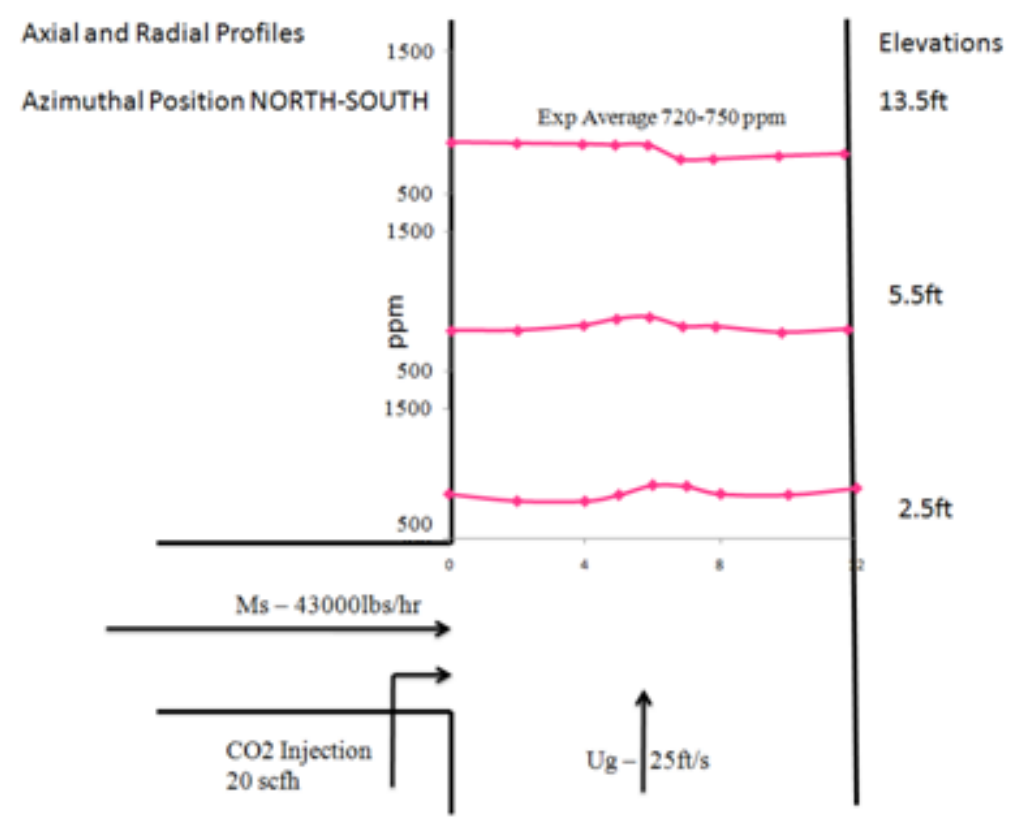

Fig 4.16 Radial dispersion of the tracer gas in the north south direction for intermediate solids feed 


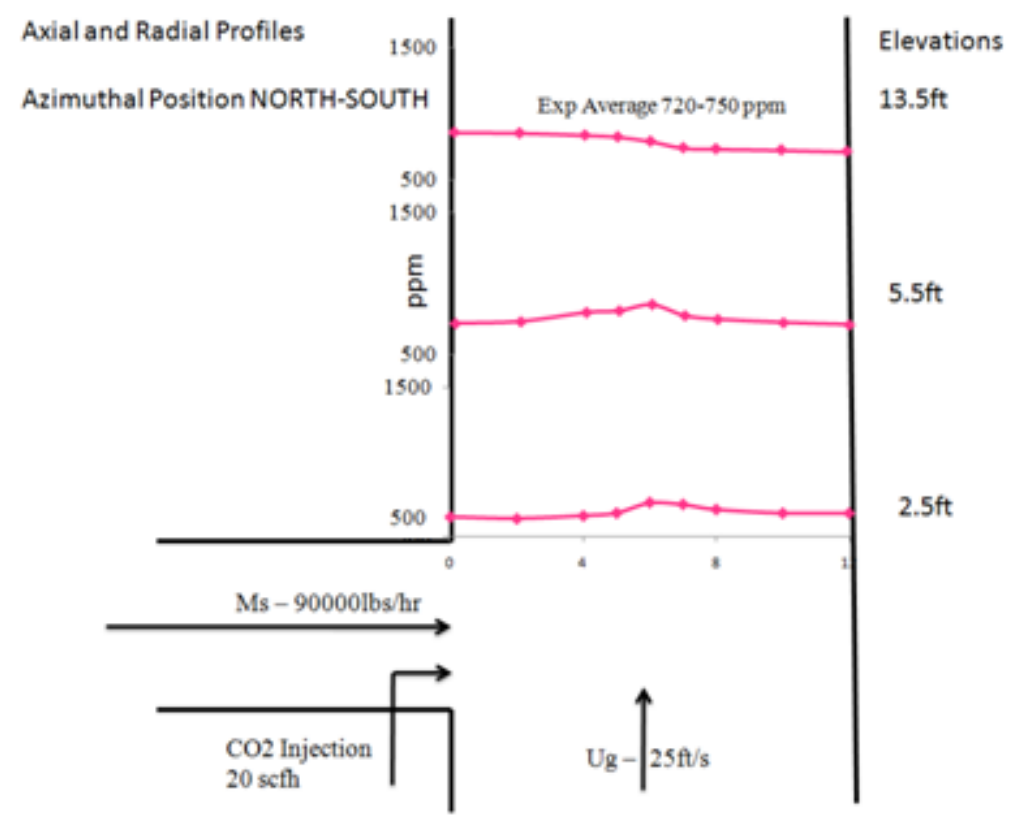

Fig 4.17 Radial dispersion of the tracer gas in the north south direction for high solids feed

\subsubsection{Analysis of the Results}

Figures 4.13 to 4.17 show the radial dispersion of the tracer gas. These results are for three levels of solids circulation rates $\left(G_{s}\right)$, low, intermediate and high and for three levels of superficial gas velocities $\left(\mathrm{U}_{\mathrm{g}}\right)$ low, intermediate and high. The $\mathrm{CO}_{2}$ tracer gas is injected into the stream of solids coming through the L-Valve at 20 SCFH. A couple of prominent observations that can be made are that for all elevations especially at the low elevations, in the east west directions, the results follow the pattern anticipated in figures 4.8, 4.9 and 4.10. At low momentum for the solids stream entering the riser the concentration of the tracer gas is highly concentrated at the entry wall. For an intermediate momentum for the solids stream entering the riser, the concentration of the tracer gas levels out across the riser cross section. And for a high momentum for the solids stream entering the riser the concentration of the tracer gas, though more leveled than the low momentum case shows a higher value on the opposite side of the wall than at entry wall. For the results of the North South measurements, we can clearly see that for a low momentum of the solids stream entering the riser the tracer gas concentration is highly parabolic. The tracer gas concentration profile keeps leveling out as the momentum of the solids stream entering the riser keeps increasing from a low to an intermediate to a high value.

These observed trends go well with the results obtained by Bi el al (1992) as indicated in chapter 3 (figure 3.31) where ozone concentrations transition towards a flatter level profile as the 
solids concentration increases. These trends also match the results obtained by Werther et al (1999) shown in chapter 3 (figures 3.33 to 3.35). Here to the concentration of the tracer gas transitions from a skewed profile to a well distributed profile as the solids concentration increases. In addition it is quite evident from the tracer gas measurements made in the NorthSouth direction that there is not a significant influence of any tracer gas backmixing along the wall. A lack of a higher concentration values at the wall followed up by a dip in the trend next to the wall show that there's no effect of tracer gas backmixing in the dense bed region.

This tracer gas technique is primarily being used as a tool to study the distribution of a stream of solids entering the riser for the lower levels of the dense entry region. At higher elevations, the solid stream will have had disintegrated thus the information obtained at those elevations cannot be used to estimate the distribution of the solids stream entering the riser. From the tracer gas results obtained in the east-west and north-south directions, it is quite clear that at the low elevations, for a low momentum of the solids stream entering the riser, the injected solids are concentrated at the entry wall as indicated by a higher tracer concentration value. As the momentum of the solids stream entering the riser increases, the distribution of the solids becomes more uniform across the riser. At a high momentum of the solids stream entering the riser, the solids distribution shows a higher concentration at the opposite wall from the L-Valve though overall the solids distribution is quite uniform as indicated by the tracer concentrations.

As seen from the figures, there is a 5\% error shown for each radial measurement in for the abscissa and mantissa. Thus there is a 95\% confidence in the measurements made at each radial position for all elevations and azimuthal positions. The confidence interval was calculated for the center point as 8 repeat measurements were made for the center point. The confidence interval calculated for the center point was assumed to be true for all other radial positions.

Before we go further a key aspect here is that for measurements made in the east west direction the concentration profiles are Skewness-sensitive where bias of the profile is the important parameter. The skewness values for the measurements made at 0.762 and 4.115 meter in the East West direction are given in tables 4.1 and 4.2 respectively.

\begin{tabular}{|c|c|}
\hline Solids Momentum & Skewness \\
\hline Low & 1.2264 \\
\hline Intermediate & 0.1195 \\
\hline High & -1.0244 \\
\hline
\end{tabular}

Table4.1 Skewness values for 0.762m in the East West Direction 


\begin{tabular}{|c|c|} 
Low & 0.1024 \\
\hline Intermediate & -0.1218 \\
\hline High & -0.1536 \\
\hline
\end{tabular}

Table4.2 Skewness values for $4.115 \mathrm{~m}$ in the East West Direction

Skewness measure indicates the level of non-symmetry. If the distribution of the data is symmetric then skewness will be close to 0 (zero). The further from 0 , the more skewed the data. A negative value indicates a skew to the left. To analyze if the skewness is significant or not we need to compare the skewness numbers to the standard error of skewness. The standard error of skewness is given by the equation

$$
\text { Std. Error of Skewness }=\sqrt{\frac{6}{N}}
$$

If the skewness is more than twice the standard error of skewness than it indicates that the skewness is significant. For all three momentum rates the skewness of the tracer gas distribution is not significant for both elevations. However the magnitude of the skewness decreases with elevation. The best case scenario is the intermediate momentum of the solids stream coming into the riser as it has the lowest magnitude of skewness for both elevations.

The skewness values indicate a couple of things. A high skewness at a point indicates that the solids stream entering the riser is concentrated at that point especially at the lower dense entry regions. This leads to a poor mixing and is caused due to a lack of solids momentum which in turn leads to low turbulence in the system.

Kurtosis is a measure of the peakedness of the data. Again, for normally distributed data the kurtosis is 0 (zero). As with skewness, if the value of kurtosis is too big or too small, there is concern about the normality of the distribution. To analyze if the kurtosis is significant or not we need to compare the kurtosis numbers to the standard error of kurtosis. The standard error of kurtosis is given by the equation

$$
\text { Std. Error of Kurtosis }=\sqrt{\frac{24}{N}}
$$

The kurtosis values for the measurements made at 0.762 and 4.115 meters in the East West direction are given in tables 4.3 and 4.4 respectively.

\begin{tabular}{|c|c|}
\hline Solids Momentum & Kurtosis \\
\hline Low & 1.3720 \\
\hline Intermediate & 0.3011 \\
\hline High & 0.3087 \\
\hline
\end{tabular}


Table4.3. Kurtosis values for $0.762 \mathrm{~m}$ in the North South Direction

\begin{tabular}{|c|c|}
\hline Solids Momentum & Kurtosis \\
\hline Low & 0.5323 \\
\hline Intermediate & 0.1841 \\
\hline High & 0.1389 \\
\hline
\end{tabular}

Table4.4. Kurtosis values for $4.115 \mathrm{~m}$ in the North South Direction

If the kurtosis is more than twice the standard error of skewness than it indicates that the distribution of the data is non-symmetric. The standard error of kurtosis is 1.07. For all three momentum rates the kurtosis of the tracer gas distribution is not significant for both elevations. However the magnitude of the kurtosis decreases with elevation. The best case scenario is the intermediate momentum of the solids stream coming into the riser as it has the lowest magnitude of kurtosis for both elevations.

The kurtosis values indicates several items to be considered. In case of the measurements made in the north/south direction the measurement axis being perpendicular to the probe, for lower solids momentum and higher superficial gas velocity a highly peaked (leptokurtic) tracer profile is observed. This is again an indication of two things. At the lower levels of the dense entry region, the solids stream entering the riser is concentrated at certain points. Second this localized concentration leads to poor mixing and is caused due to a lack of adequate solids momentum to create sufficient turbulence in the system.

At the end one may conclude that the best case scenario is the intermediate momentum of the solids stream coming into the riser. The condition yields a positive skewness but is less than twice the standard error of skewness and hence the skewness of the data is not a concern for all elevations. It also provides a platykurtic dispersion profile for all elevations and the kurtosis values for all elevations is less than twice the standard error of kurtosis, making the kurtotic profile not a concern.

An overall observation made from the dispersion profiles is that the skewness and kurtosis values transition from significantly influencing to non-influencing with an increase in elevation. This indicates that the gas is well dispersed across the riser cross section. Nothing can be mentioned about the solids dispersion since at these high elevations, the co-flow condition may or may not exist. It is quite possible that at these elevations the two streams have separated. In addition, an increase in turbulence on account of an increase in the solids momentum also led to flatter concentration profiles. 
An anomaly is observed for a particular case where the stream of solids entering the riser at low momentum is well distributed across the riser cross section at the lower levels of the dense. That is, the tracer gas profiles have a low magnitude of positive skewness in the east/west direction and more platykurtic (flat) in the north south direction. There is a specific difference in this operating condition than those for other low solids momentum conditions. The momentum of the dense bed solids and superficial gas coming from the riser base is also low in this case. This leads to a potential reason for the variations observed. At the lower elevations, a high positive skewness and a highly leptokurtic profile is seen due to a lack of sufficient momentum of the solids stream entering the riser. As result the solids stream cannot penetrate the stream of the dense bed solids and superficial air coming from the riser base. This ultimately leads to a low magnitude of turbulence produced.

Ideally a perfect mixing condition would be indicated by a flat line with $\mathrm{C} / \mathrm{C}_{0}=1.0$. Even at a height of $4.115 \mathrm{~m}$ (13.5 ft) which corresponds to a distance of approximately 14 times the riser diameter the perfect mixing condition is not achieved as can be seen from the figures.

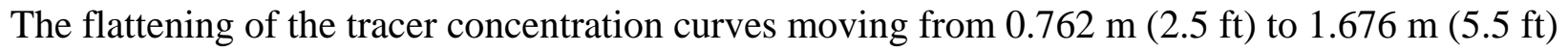

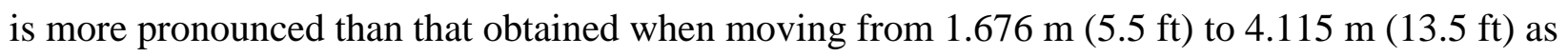
can be seen from the figures. In addition the variation in the tracer profiles from going from a low solids circulation rate to a high circulation rate is more pronounced at the lower elevations as compared to the higher elevations. As the tracer gas is dispersed the concentration gradients decrease leading to less mass transfer in the radial.

To determine that we have reached our true well mixed profile for the given system, we perform a simple test. We take the mean of the radial measurements of the tracer gas at the highest elevation for an intermediate solids momentum, which gives the best well mixing profile. We then compare this value to the expected theoretical mean at fully developed condition. Allowing for a confidence interval of the $90 \%$ we see that the two values are within the given acceptable tolerance shown below. Thus the idea is to compare the theoretical expected mean tracer concentration to the mean of the tracer concentration at the best mixed condition $(4.115 \mathrm{~m}$ and intermediate solids circulation). The experimental mean or average concentration was found to be $806.579 \mathrm{ppm}$ without baseline correction. The expected mean concentration for a 20scfh flow was calculated to be $820 \mathrm{ppm}$. Thus there is an error of less than $5 \%$ which indicates accurate measurements. 
The results presented above, can be analyzed in the context of particle turbulence interaction in gas solids flows. As the carrier phase turbulence is thought to be the main mechanism in dispersion of gas in radial direction, any change in the turbulence structure due to particle presence also affects the gas dispersion. Generally the problem in which the changes in the carrier phase turbulence due to the effect of the dispersed phase is analyzed, is known as turbulence modulation, and will be covered in Chapter V. 


\section{Chapter V}

\section{Hydrodynamic Analysis of Tracer Gas Results}

\subsection{Introduction}

Turbulent particle laden flow is a cornerstone for many industrially relevant processes such as pneumatic conveying of particulates, coal gasifier and so on. Despite their widespread applications a majority of these processes do not operate at their optimal conditions due to a lack of understanding of the intricate particle laden flow phenomenon during the process design stage. This lack of understanding leads to the difficulties in design, optimization and scale up of such processes where start-up of unit operations involving particle laden flows only has a $60 \%$ success rate compared to the $90 \%$ for other operations (Merrow et.al, 1985). One particle laden flow phenomenon of significant industrial interest is the effect of the Reynolds number variation on the modulation of the gas-phase turbulence intensity. The effectiveness of a particle laden flow is governed by the gas phase turbulent intensity, which influences the pressure drop and the gas particle interactions in the process. The presence of particles can either attenuate or enhance the gas-phase turbulence intensity with respect to its unladen value. Importantly the modulated gas phase turbulent intensity in turn influences the particle fluctuating motion resulting in a two way coupling interaction between the two phases. The turbulent intensity modulation can be attributed to two mechanisms 1) Interparticle collisions that results in a redistribution of the particle fluctuating velocity that induces velocity fluctuations in the gas phase, 2) gas particle interaction that is significant when the particle volume fraction (a) larger than 10-6 (Elghobashi, 1994). In the first mechanism the gas-phase velocity fluctuations induced by the inter-particle collisions modifies the gas-phased Reynolds stress that controls the rate of turbulence production and dissipation (Vreman, 1997) and moreover it can also enhance the fluctuating motion of the neighboring particles through a "long range" gas-particle interaction mechanism (Koch, 1990). In the second mechanism the gas- 
particle interaction leads to turbulence dissipation by means of the fluctuating drag force and/or turbulence productions by means of the vortex shedding phenomenon.

Gore and Crowe (1989) and Hestroni (1989), sought to summarize the trends in turbulence modulation by compiling turbulent fluid particle flow data available in the literature. Their compiled data included both liquid particle and gas particle/jet flows at a wide range of particle size, density and concentration. They defined the particle Reynolds number as:

$$
R e_{p}=\rho_{f} d_{p}\left(U_{f}-U_{s}\right) / \mu_{f}
$$

This was used to characterize the particle laden flows, where $d_{p}, \mu_{g}$, and $\rho_{g}$ are the particle diameter, fluid viscosity and density respectively whereas $U_{\mathrm{s}}$ and $U_{\mathrm{g}}$ are the streamwise mean (i.e. time averaged) velocities of the fluid and particle respectively.

In their work the authors used the ratio of particle diameter to a turbulent length scale, $d_{p} / l_{e}$ as an estimate of the turbulence intensity. $l_{e}$ is the characteristic length of the most energetic eddy when only one phase is present. They concluded that the turbulence intensity in the pipe core was attenuated with respect to its unladen value in the presence of small particles (low $R_{p}$ ). The turbulence attenuation was attributed to the viscous drag force exerted on the small particles travelling with the turbulent eddies. Conversely, they found that the turbulence intensity was enhanced in the presence of large particles (high $R_{p}$ ) that were irresponsive to the turbulent eddies. The turbulence enhancement was attributed to the vortex shedding phenomenon in which instability in the wake region associated with the large particles at $\operatorname{Re}_{\mathrm{p}}>400$ (Hetsroni, 1989) enhanced the gas-phase velocity fluctuations. Gore and Crowe (1989) proposed that the ratio of the particle diameter to the turbulence length scale could be used as an indicator to predict whether turbulence attenuation or augmentation would occur. In summary the found that the ratio $\mathrm{d}_{\mathrm{p}} / l_{\mathrm{e}} \approx 0.1$ provides an estimate of whether the relative turbulent intensity of the carrier phase will be increased or decreased by the addition of the second phase when particle fluid interactions are dominant. They noted that the critical diameter/length scale ratio refers to only the question of 
increasing or decreasing the turbulent intensity and does not relate to the magnitude of the change. The amount of change will be affected by various parameters such as, concentration, density ratio, flow Reynolds number and flow configuration. The strongest evidence for this trend has been observed for gas solid flows in pipes and jets.

In addition to the particle size, increasing the particle loading was also found to intensify the degree of the gas phase turbulence modulation for both large and small particles (Tsuji et.al, 1984). The particle loading (m) is defined as the ratio of the particle mass flow rate to that of the gas. Moreover, particle laden flows often give rise to particle clustering in which a collection of particles form meso-scale structures that occur at a length scale larger than the individual particle scale. The particle clustering phenomenon tends to occur in a highly concentrated particle laden flow in which the loss of particle fluctuating energy due to the inelastic interparticle collision is significant. Importantly the mesoscale structures exhibit a highly turbulent nature as a result of the unsteady formation and dissociation of the clusters (Agrawal et.al, 2001), which modulates the gas-phase turbulence intensity. Lastly particle rotation which is typically generated due to the interparticle collision, particle wall collision or the mean shear flow across the particle can create wake instability (i.e., vortex shedding) in liquid particle flows for $\mathrm{Re}_{\mathrm{p}}<400$ resulting in turbulence enhancement or augmentation (Best, 1998).

In the work done by Hadinoto and Curtis (2009), the particle Reynolds number is defined as

$$
R e_{p}=2 \rho_{g} U_{s g} R / \mu_{g}
$$

Where $\mathrm{R}$ is the pipe radius and $\mathrm{U}_{\mathrm{sg}}$ is the mean superficial velocity of the gas in the axial direction. The gas phase turbulence intensity $(\sigma)$ is defined as the gas phase velocity variance normalized by the square of the axial mean velocity of the unladen flow at the pipe center $\left(\mathrm{U}_{0}\right)$. Other work on turbulence modulation in gas-particle vertical flows were limited to experimental studies on the effects of particle physical properties and particle concentrations (Tsuji et.al, 1984; Agrawal et.al, 2001; Best, 
1998; Kasagi et.al, 1995; Lee et.al, 1982; Jones, 2001). Most of the work mentioned, reported the turbulence modulation based on a constant particle Reynolds number.

For studies in which the Reynolds number was varied, the particle size and loading radios were also varied. As a result the isolated effects of varying the flows length and velocity scales on the turbulence modulation were never examined. For this reason Hadinoto et. al, (2005) conducted a gas particle pipe flow experiment to investigate the effect of the particle Re number variation on turbulence modulation. They varied Re between 6000 and 24000 by varying the mean superficial velocity between 8 and $22 \mathrm{~m} / \mathrm{s}$ while keeping the particle loading constant. The experiment was conducted in a vertical downward pipe flow using 70 and $200 \mu \mathrm{m}$ glass beads with particle density of $2500 \mathrm{~kg} / \mathrm{m}^{3}$. For both 70 and $200 \mu \mathrm{m}$ glass particles Hadinoto et.al, (2005) reported that the gas phase turbulence intensity in the pipe core increased with an increase in $\mathrm{Re}_{\mathrm{p}}$, which resulted in turbulence enhancement relative to the unladen flow at high $\operatorname{Re}_{\mathrm{p}}(>13000)$.

The turbulence enhancement in the presence of small glass beads at high $\operatorname{Re}_{\mathrm{p}}$ and at a low particle loading was also reported by Maeda et.al, (1980). The trend in the turbulence intensity as a function of $\operatorname{Re}_{\mathrm{p}}$ for the gas particle flow was in contrast to that of the unladen flows. Here the turbulence intensity in the pipe core had been found to decrease with an increasing $\operatorname{Re}_{\mathrm{p}}$ (Fluent users guide book, 2001). Using only the mean slip velocity data Hadinoto et.al, (1985) postulated that a collective particle effect in which multiple neighboring particles move with non zero slip velocities might have contributed to the turbulence enhancement at high $\mathrm{Re}_{\mathrm{p}}$.

The enhanced turbulence in the presence of large particles can be explained by the vortex shedding phenomenon. It is known that when

$$
R e_{p}=\frac{\left(U_{g}-U_{p}\right) * d}{v_{g}} \leq 110
$$

there is no vortex shedding downstream of the particle. Achenbach (1974) has shown that vortex shedding occurs as $R e_{p}>400$. In his experiments vortex shedding occurred in the range $400<R e_{p}<1000$ with a Strouhal number of $S=0.2$ where

$$
S=f_{w} d / u_{r}
$$


$f_{w}-$ frequency of the vortex shedding, $d_{p}$ - diameter of the sphere, $u_{g}-$ free stream velocity. For $1000<R e_{f_{w}}<10,000$ he found $\mathrm{S}=2.0$. An examination of the data by Tsuji et.al (1984, 1988; Tsuji and Morikawa 1982) (figure 5.2) reveals that small particles $d_{p}=200 \mu m, R e_{p} \approx O(0.10)$ always caused a suppression of turbulence of the mainstream. Large particles $\left(\mathrm{d}_{\mathrm{p}}=300 \mu \mathrm{m}\right), R e_{p} \approx O(1000)$ always caused an increase in the turbulence intensity in the mainstream as shown in figure 5.1.

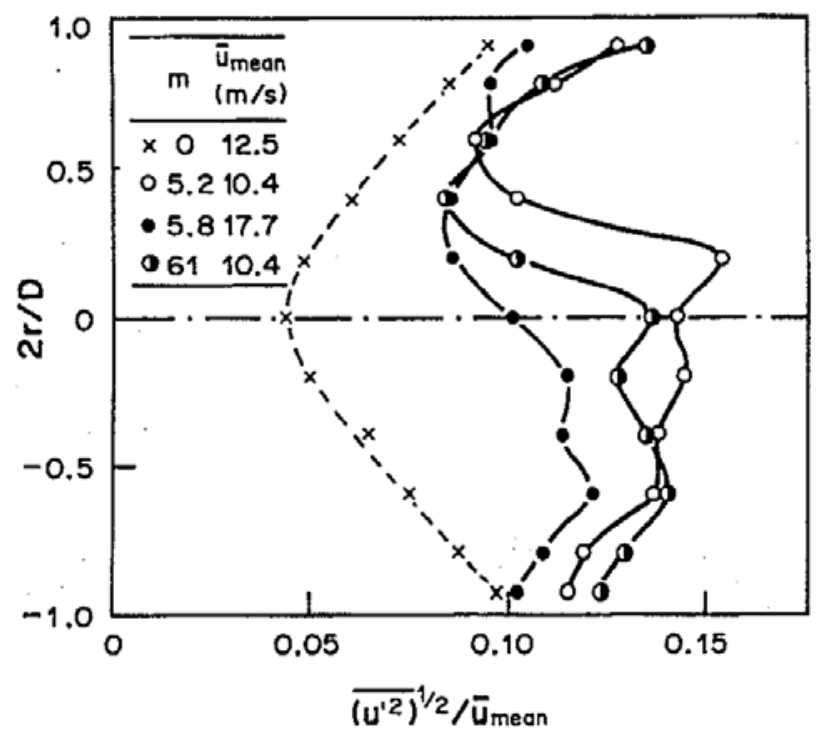

Fig5.1 Turbulence intensity of air in presence of $3.4 \mathrm{~mm}$ particles in a $30 \mathrm{~mm}$ dia. horizontal pipe

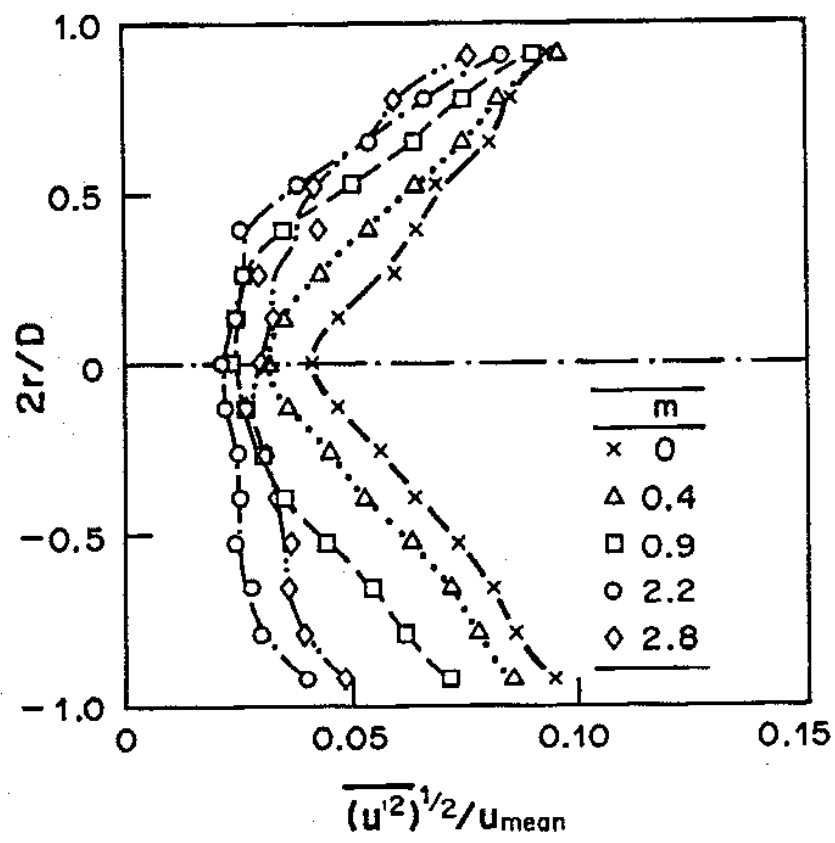


Fig.5.2. Turbulence intensity of air in presence of $0.2 \mathrm{~mm}$ particles in a $30 \mathrm{~mm}$ dia. horizontal pipe

This concept of turbulence modulation has been applied to jets. The L-valve feeding the solids into the riser, can be considered as a large jet. In one of the earliest measurements by Hetsroni and Sokolov in 1971, the properties of an air jet laden with $13 \mu \mathrm{m}$ droplets were measured. They found that the turbulence intensity was uniformly reduced by small particles i.e. it was decreased almost proportionally to the loading of the droplets. Tsuji et.al, (1988) using a one dimensional LDA measured the motion of the particles in an air jet. They too realized that effect of smaller particles is to decrease the intensity of turbulence. They did not see a significant effect of large particles - primarily because the loading was very light but the large particles did increase the intensity of turbulence in some parts of the jet. Levi and Lockwood (1981) using a one dimensional LDA measured sand particles in free downward air jet. Here too they found that larger particles of $850-$ $1200 \mu \mathrm{m}$ caused a significant increase in the turbulence level, whereas smaller particles of 180 - $250 \mu \mathrm{m}$ clearly caused a suppression of turbulence.

The jet or the L-valve feeds the solids into the riser. The riser geometrically is a vertical pipe. Tsuji (1984) investigated vertical pipe flow. He found that the smaller the particles, the flatter the mean velocity distribution. He also found that large particles possessing a high particle Reynolds number increased the air turbulence throughout the pipe cross section, whereas small particles with low Reynolds number reduced the air turbulence.

It is now accepted that for dilute two-phase flows, the presence of the particles can either dampen or augment the gas phase turbulence depending on the gas and particle properties (Tsuji et al., 1984; Hetsroni, 1989; Gore and Crowe, 1989; Elgobashi, 1994; Kulick et al., 1994) However, there is no general consensus about the criterion which determines the borderline and extent of the suppression and augmentation of gas phase turbulence with the addition of particles. Some experimental data suggest an increase in turbulence with addition of particles (Tsuji and Morikawa, 1983; Yokuda, 1990) whereas some results indicate the 
opposite trend (Schreck and Kleis, 1993). It should also be noted the fluctuations in one direction can be more affected by the presence of the particles than the fluctuations in the other direction (Tsuji et al. 1984).

Gore and Crowe (1989) gathered data from a variety of researchers in order to find a trend for turbulence modulation. They suggested that the ratio of the particle diameter to length scale of the largest eddy, $l_{\mathrm{e}}$ (scale of the most energetic eddy) could be used as the key parameter determining the turbulence modulation. When this ratio is smaller than 0.1 , the data shows that the turbulence level decreases with the addition of particles whereas an increase is observed for ratios greater than 0.1. The physical explanation is as follows: the small particles which are much smaller than the large scale eddies responsible for the turbulent transport follow the eddies they interact for at least part of their life time. Part of the eddy's energy is imparted to the particles since the eddy, through the drag force, is moving the particle. The turbulent energy of the eddy is therefore transformed in to the kinetic energy of the particle and the turbulent intensity is reduced. Large particles $\left(d_{p} / l_{e}>0.1\right)$, on the other hand, tend to create turbulence in their wake near the scale of the large scale eddies, thus increasing the turbulent intensity of the gas. Hestroni (1989) postulated a similar theory by performing an order of magnitude analysis, stating that the particles with Reynolds numbers greater than 400 would augment the turbulence due the generation of turbulence by vortex shedding at their wakes and those with Reynolds numbers less than 400 would attenuate it.

Zheng et al. (1992) observed that the radial dispersion coefficients decreased with an increase of the solids volume fraction. Arena (1997) suggests that for higher solids volume fraction values, clustering effects become significant and a cluster having an effective diameter larger than a single particle might increase the turbulence due to the wake effects leading to a well mixed profile. The theories of Gore and Crowe (1989) and Hestroni (1989) depend on data from relatively dilute two-phase flows, including gas-liquid flows. Secondly, these theories emphasize the transition from single phase to two-phase flow, rather than the variation of the turbulence intensity with the volume fraction of the discrete phase. Thirdly, the 
nature of gas turbulence and particle interactions in dense gas-solid flows is not really well known as in case of dilute flows (Elgobashi, 1994). Therefore, it would be more appropriate to state that in case of dense CFB risers, the solids alter the gas phase flow and turbulence in such a way that, the radial gas mixing increases with increasing solids loading. Some of the studies that contradict these trends are cited here, For instance, Werther et al., (1992a) found no difference in radial dispersion coefficients at a superficial gas velocity of $3 \mathrm{~m} / \mathrm{s}$ and the solids circulation rate changing between $0-70 \mathrm{~kg} / \mathrm{m}^{2} \mathrm{~s}$, although the solids concentration at the measurement location changed between 0-1\%. Recently, Sterneus et al., (2000) published the results of their gas mixing experiments carried out in a rectangular cross-section riser. They stated that a very dilute flow gives about the same dispersion as the single phase flow. Increasing the solids holdup at constant velocity increased the dispersion coefficient to a plateau value after which no change in dispersion coefficient was observed. As they indicated, the increase in the dispersion coefficient with loading was possibly due to the large scale motions and fluctuations caused by the bubbles at the bottom of the bed, which is not usually observed in lab scale risers with high values of height-to-diameter ratio.

In a very recent study conducted by Kartusinsky et.al, (2009) they looked into the variation of mass loading and particle density in gas - solid particle flow in pipes. They also found that as seen in figure 5.3, there is an increase in turbulence intensity of the air for larger particles and correspondingly a decrease in the turbulence intensity due to small particles. 


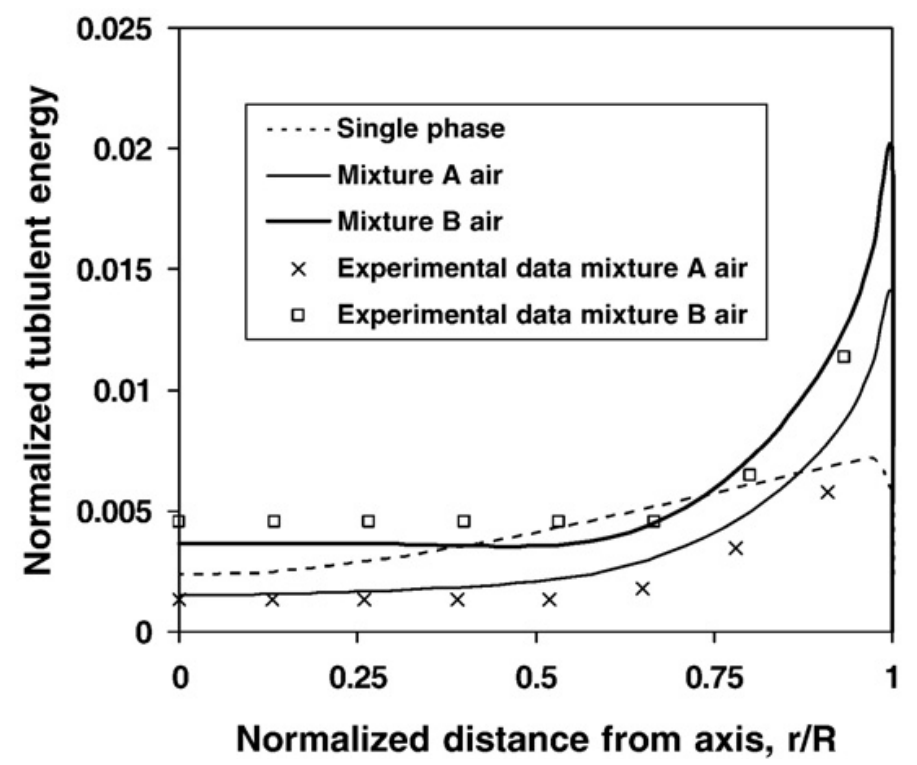

Fig.5.3. Profiles of turbulence intensity of air carrying solids [Kartusinsky, (2009)]

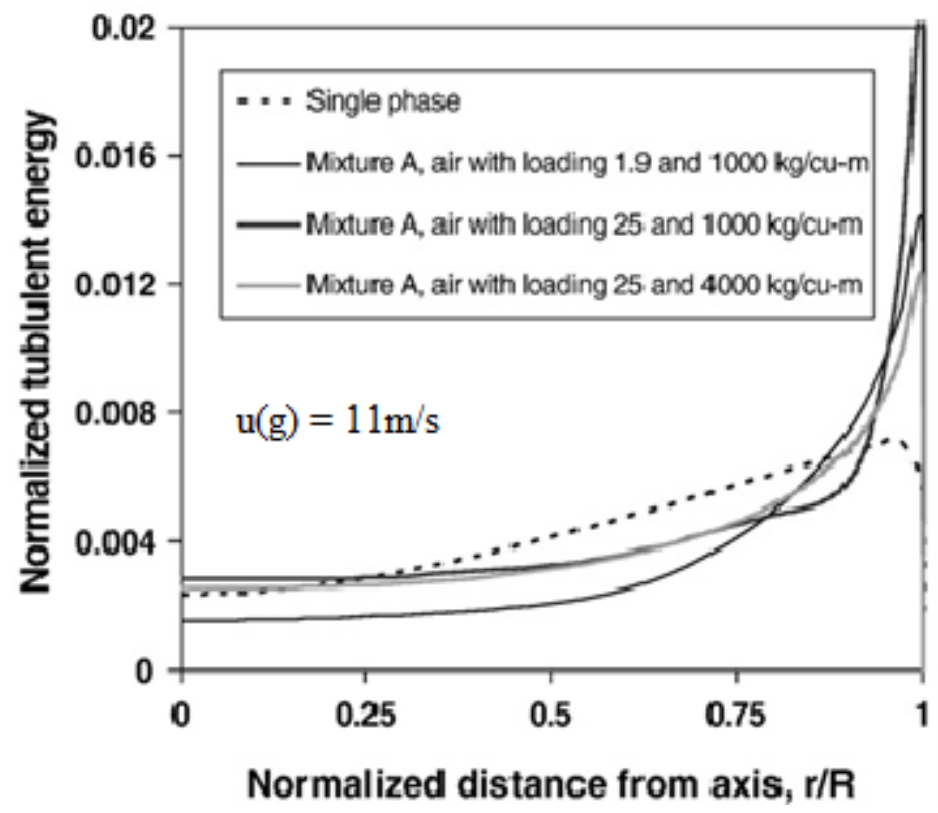

Fig.5.4. Effect of loading and particle density on turbulent intensity [Kartusinsky, (2009)]

They also found as evident from figure 5.4, that an increase in the solids loads led to an increase in the turbulent intensity whereas the density change had no significant effect on the turbulent intensity except near the pipe boundary. In their work Mixture A, is composed of equal parts (33.3\%) of smaller particles with diameters $\mathrm{d}_{1}=219 \mu \mathrm{m}, \mathrm{d}_{2}=243 \mu \mathrm{m}$ and $\mathrm{d}_{3}=264 \mu \mathrm{m}$, material density $\rho_{\mathrm{p}}=1000$ 
$\mathrm{kg} / \mathrm{m}^{3}$ and loading $\mathrm{m}=1.9 \mathrm{~kg} / \mathrm{kg}$. Mixture $\mathrm{B}$, is composed of equal parts $(33.3 \%)$ of larger particles with diameters $\mathrm{d}_{1}=450 \mu \mathrm{m}, \mathrm{d}_{2}=500 \mu \mathrm{m}$ and $\mathrm{d}_{3}=550 \mu \mathrm{m}$, material density $\rho_{\mathrm{p}}=1000 \mathrm{~kg} / \mathrm{m}^{3}$ and loading $\mathrm{m}=3.4 \mathrm{~kg} / \mathrm{kg}$.

Mathematically there are several models have been proposed to explain this observed trends. Yuan and Michaelides (1992) proposed a model in which the velocity defect in the wake is responsible for the augmentation of the turbulence and the work associated with the motion of the particulate phase is responsible for the attenuation of turbulence. They suggest that the turbulence generation is given by

$$
G_{s}=d_{p}^{2} \rho_{c} f\left(l_{w}\right)\left(U_{g}^{2}-V_{p}^{2}\right)
$$

Where $d_{p}$ is the particle diameter, $\rho_{g}$ is the density of the carrier phase, $f\left(l_{\mathrm{w}}\right)$ is the function of the wake size and $U_{g}$ and $V_{p}$ are fluid and particle velocities respectively. From the above equation we can see that there is direct proportionality between the turbulence generation and the particle diameter. Thus larger particles cause turbulence augmentation and smaller particles cause turbulence attenuation. More recently Kenning and Crowe (1997) proposed a model for turbulence modulation for gas particle flows based on the work done by particle drag and the dissipation based on the length scale corresponding to the interparticle spacing. They stated that the generation rate of the turbulence energy per unit mass of mixture is given by

$$
G_{s}=\frac{f}{\tau_{v}} C\left(U_{g}-V_{p}\right)^{2}
$$

Where $\mathrm{f}$ is the ratio of the drag coefficient to Stokes drag, $\mathrm{C}$ is the ratio of mass of the dispersed phase to the carrier phase in a two-phase mixture and $\tau_{v}$ is the particle response time. Thus from this equation we can see that there is direct proportionality between the amount of turbulent energy generated and the volume fraction. A higher solids volume fraction leads to an augmentation of the turbulent energy intensity and a lower solids volume fraction leads to an attenuation of the turbulent energy intensity, for a constant $d_{p}$.

Michaelides (2006) lists six mechanisms for turbulence modulation due to the presence of a discrete phase; which is applicable to solids particles. These are; 
a) Vortex Breaking and dissipation of turbulent kinetic energy on the surface of the immersed objects

b) Modification of the effective viscosity of the fluid

c) Eddy energy dissipated on the acceleration and deceleration of the elements of the dispersed phase

d) Wakes and shedding of vortices behind immersed objects

e) Fluid moving with immersed objects or being displaced by them

f) Enhancement of fluid velocity gradients between two neighboring immersed objects

The first three mechanisms increase the dissipation of the turbulence energy and the last three increase the production of the turbulent kinetic energy. Of the six mechanisms listed Peirano and Leckner (1998) state that b) and f) may not be significant for dilute suspensions. Peirano and Leckner (1998) and Elgobashi (1991) classified the coupling between particles and the turbulence of the surrounding flow into three categories based on the volume fraction of the particles or the relative distance between the particles and the ratio of the flow and particle time scales. This coupling is shown in figure 1.2. $\tau_{\mathrm{C}}$ is the characteristic time scale of the turbulent eddies in the continuous phase according to the commonly used $k-\varepsilon$ turbulence model

- $\tau_{\mathrm{K}}$ is the Kolmogorov time scale, which describes the smallest time scales of the turbulence in the continuous phase.

- The kinetic energy of the turbulence is $k$ and $\varepsilon T E$ is the dissipation of the turbulent energy.

- The third time scale in the diagram is $\mathrm{tv}$ which is the particle relaxation time (Peirano and Leckner, 1998)

$$
\tau_{C}=C_{\mu} \frac{k}{\varepsilon} \quad C_{\mu}=0.09 \quad \tau_{K}=\left(\frac{v}{\varepsilon}\right)^{\frac{1}{2}} \quad S t=\frac{\tau_{V}}{\tau_{S}}
$$


- With a very low particle phase volume fraction, the inertia of the discrete phase is low and has little effect on the turbulence of the carrying fluid. This is called a "one - way" coupling by Elgobashi (1991).

- As the volume fraction of the particles increases to around $10^{-6}$, the discrete phase begins to modulate the turbulence through the six mechanism listed earlier, creating a "two - way coupling" between the phases.

- With high Stokes numbers the particles enhance the turbulence and at low Stokes numbers, the dissipation is enhanced.

- The region of increasingly dense suspension $\left(>10^{3}\right)$, where the collisions between the particles becomes important is characterized by "four - way coupling" with increasing complexity (Peirano and Leckner, 1998)

- The shaded part of the diagram is the area of interest. It is here where this work differs from the existing work done on gas solids mixing. Most of the work done is for dilute conditions and in the fully developed regime. Because of the low flux and low density of the operating conditions in contemporary work, they were always in the "one - way coupling" and hence did not see any effect of turbulence or modulation of turbulence.

- This work is for the dense entry region of a high flux and high density riser,. The solids suspension values for the low, intermediate and high solids feed rate is given below;

$$
\frac{\mathrm{M}_{\mathrm{S}}}{\mathrm{M}_{\mathrm{g}}}=\left(\begin{array}{c}
0.747 \\
2.468 \\
4.33
\end{array}\right)
$$

- Based on the solids fraction, we are opering in the "four - way coupling" region unlike most of the work done. 
- According to Peirano and Leckner, the effects of the particle presence on dissipation and production of turbulence are governed by redistribution of the turbulent kinetic energy spectrum, rather than uniform mechanisms over the whole spectrum.

- With medium sized particles $(500-1000 \mu \mathrm{m})$, the mean size being the particle used in this study, it was found that they increased the intensity of the turbulence in the core section but reduced it at the walls.

- These results have been theoretically predicted by Derevich (1988) since then (Peirano and Leckner, 1998).

\subsection{Understanding the Radial Tracer Gas Profiles}

The tracer gas profiles we observe cannot be explained completely by either of the two factors, i.e. momentum of the solids stream entering the riser or turbulence modulation. The results however can be explained combining the two factors i.e. a combination of the solids momentum theory and the modulation of turbulence theory.

Thus the observed results can be explained as

\section{OBSERVED RESULTS = SOLIDS MOMENTUM + TURBULENCE MODULATION}

How do we know that there is turbulence modulation? For turbulence modulation to occur for a given particle, the Reynolds number for the particle must be

$$
R e_{p}=\frac{\left(U_{p}-U_{g}\right) * d}{v_{p}} \leq 110
$$

The superficial gas velocity $\left(U_{g}\right)$ is known and the particle velocity $\left(U_{p}\right)$ is determined from the fiber optic measurements given in figure 5.5. The particle Reynolds number for both low and high velocities for a particle size of 850 microns is calculated. For the low velocity case, the $\left(R e_{p}\right)$ was determined to be 20.685 while 
the $\mathrm{Re}_{\mathrm{p}}$ was determined to be 212.568 for the high velocity case. This clearly shows that the High Density Poly Ethylene (HDPE) beads cause turbulence modulation at high circulation rates.

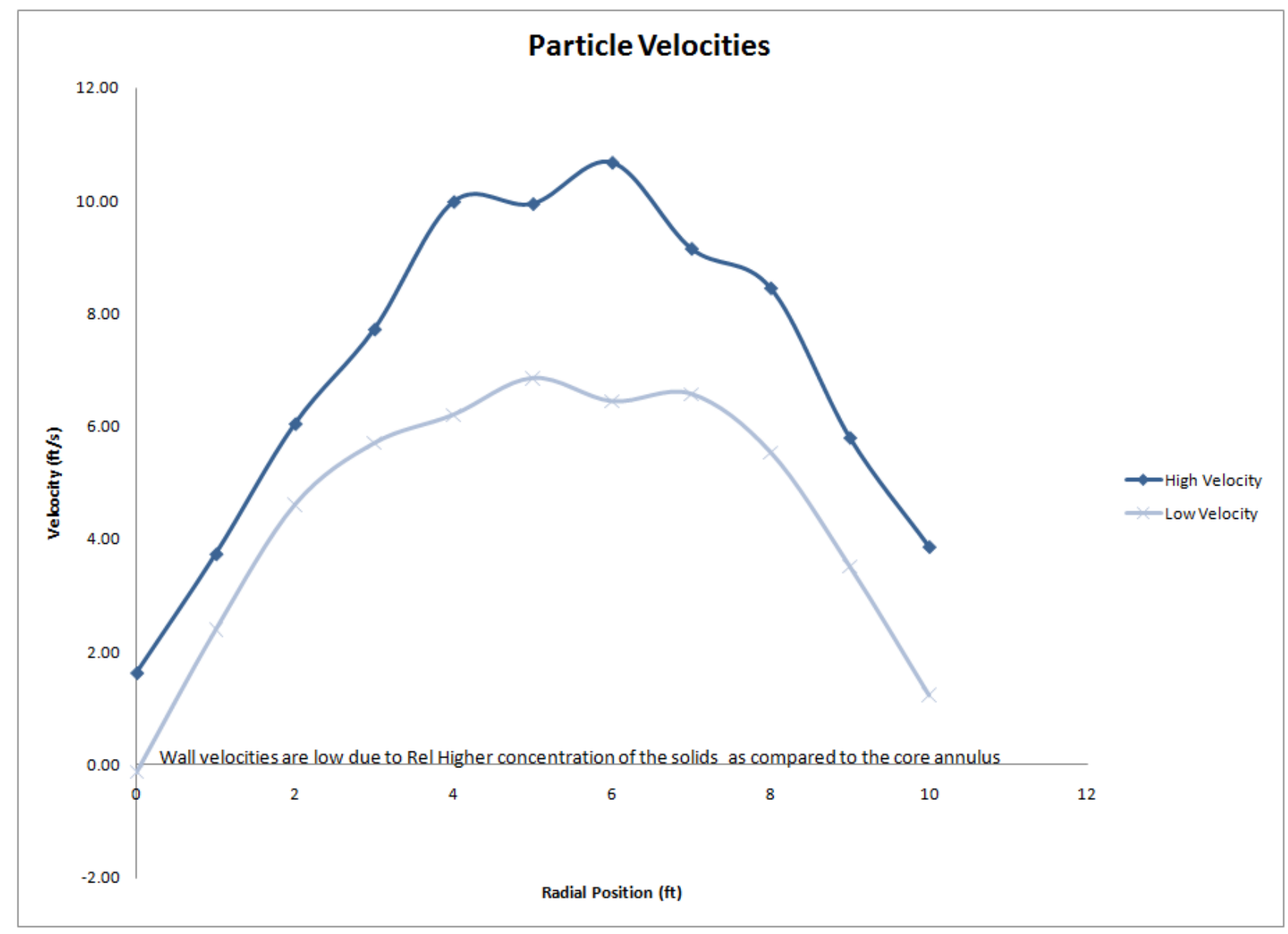

Fig5.5 Fiber optic measurements made in this work for determining the particle velocity

- Based on the solids momentum theory we have three scenarios;

- Scenario 1: The stream of solids coming from the L-Valve does not have enough momentum to enter the superficial gas jet core (low solids momentum).

- Scenario 2: The stream of solids has enough momentum to reach the center of the superficial gas jet core (intermediate solids momentum). 
- Scenario 3: The stream of solids has enough momentum to flow all the way across the superficial gas jet core till the opposite riser wall (high solids momentum).

- Now depending on the above three scenarios, we have the following cases

- Case 1-no modulation of turbulence as the solids do not enter the continuous phase

- Case 2 - modulation of turbulence achieved

- Case 3-modulation of turbulence achieved

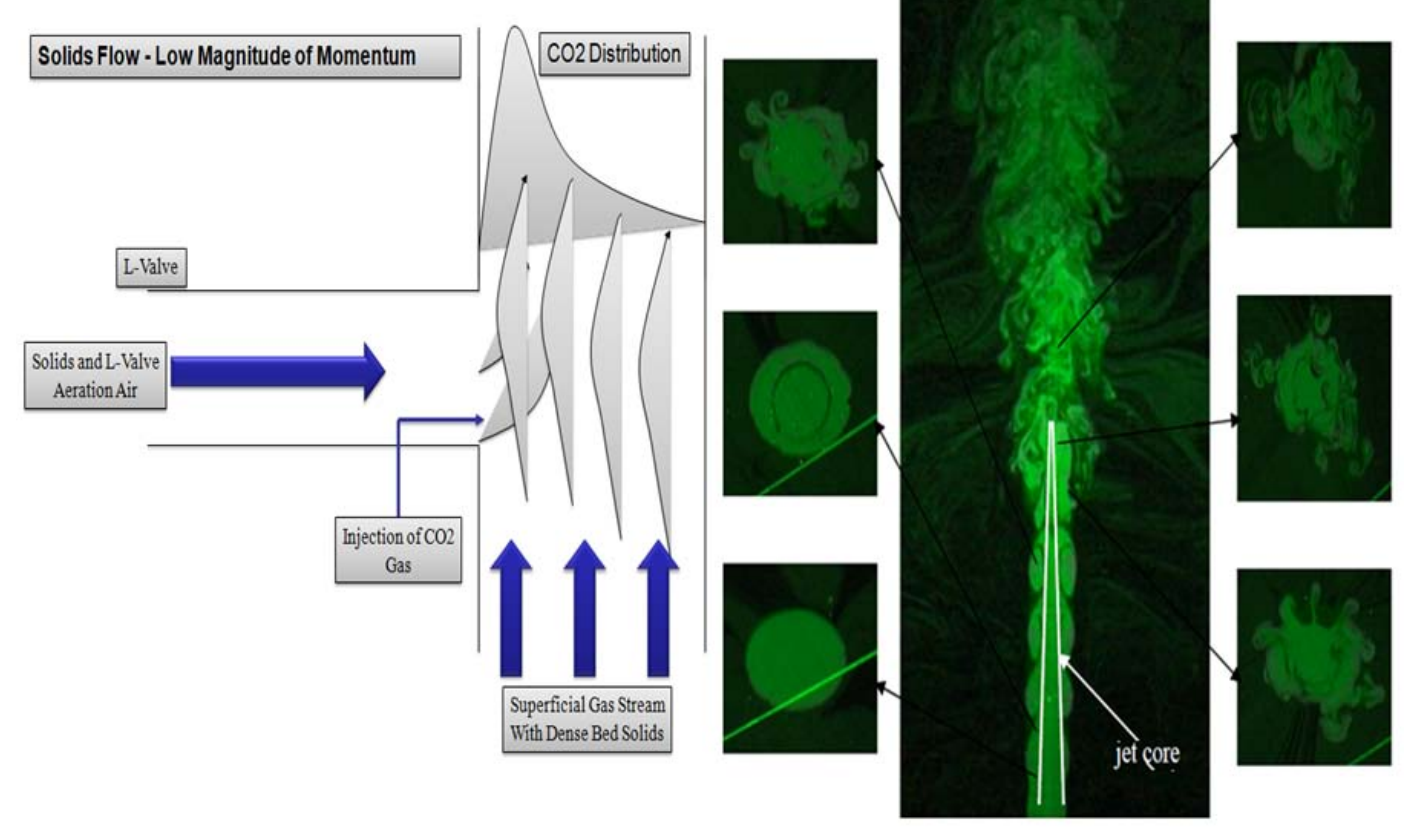

Fig 5.6 Scenario/Case 1 Gas Phase Free Jet

\subsubsection{Scenario 1: No Modulation of Turbulence as the solids do not enter the continuous phase core (Superficial Gas Stream Core)}

- In this scenario since the solids coming from the L-Valve don't have enough momentum to penetrate the superficial gas jet. Hence the superficial gas jet is like a gas phase free jet as shown in figure 5.6. 
- The transition process from the laminar to the turbulent flow is also shown in figure 5.6. The initial vortex rings roll up close to the nozzle exit.

- When the vortex rings develop downstream, the undulating ejections generate in the radial direction, which changes the regular pattern of vortex rings and finally results in the formation of stream-wise structures.

- When the stream-wise structures are broken up, the flow finishes the transition process from the laminar to the turbulence. The vortex pairs are also observed in the pictures.

- The pictures in the radial-cross sections show the mixing between the jet fluid and the ambient fluid. The potential core is filled by the dyed fluid, appearing light and the un-dyed ambient fluid appears dark.

- The ambient fluid is entrained by the jet vortex ring into the jet, whereas the jet core fluid is ejected into the ambient fluid far away which induces the jet diffusion in the radial direction.

\subsubsection{Scenario 2 and 3: Modulation of Turbulence as the solids enter the continuous phase core (Superficial Gas Stream Core)}

- Figure 5.7 compares the vortex structures of the free shear gas jet at the longitudinal cross section with those when a particle is fixed in the flow field.

- The particles are placed in the potential core region of the jet, mimicking the process of scenarios 2 and 3 of the NETL riser, where the solids now have enough momentum to penetrate the core of the superficial gas stream. Here the characteristic flow structures are rolling up vortex rings.

- There are two main influences of particle in the free shear jet flow field. Firstly, the particle changes the flow passage, which induces the flow instability earlier. 
- Secondly, for the particles in diameter larger than $10 \mathrm{~mm}$, the wakes are formed behind them, but no wakes are behind the smaller particles.

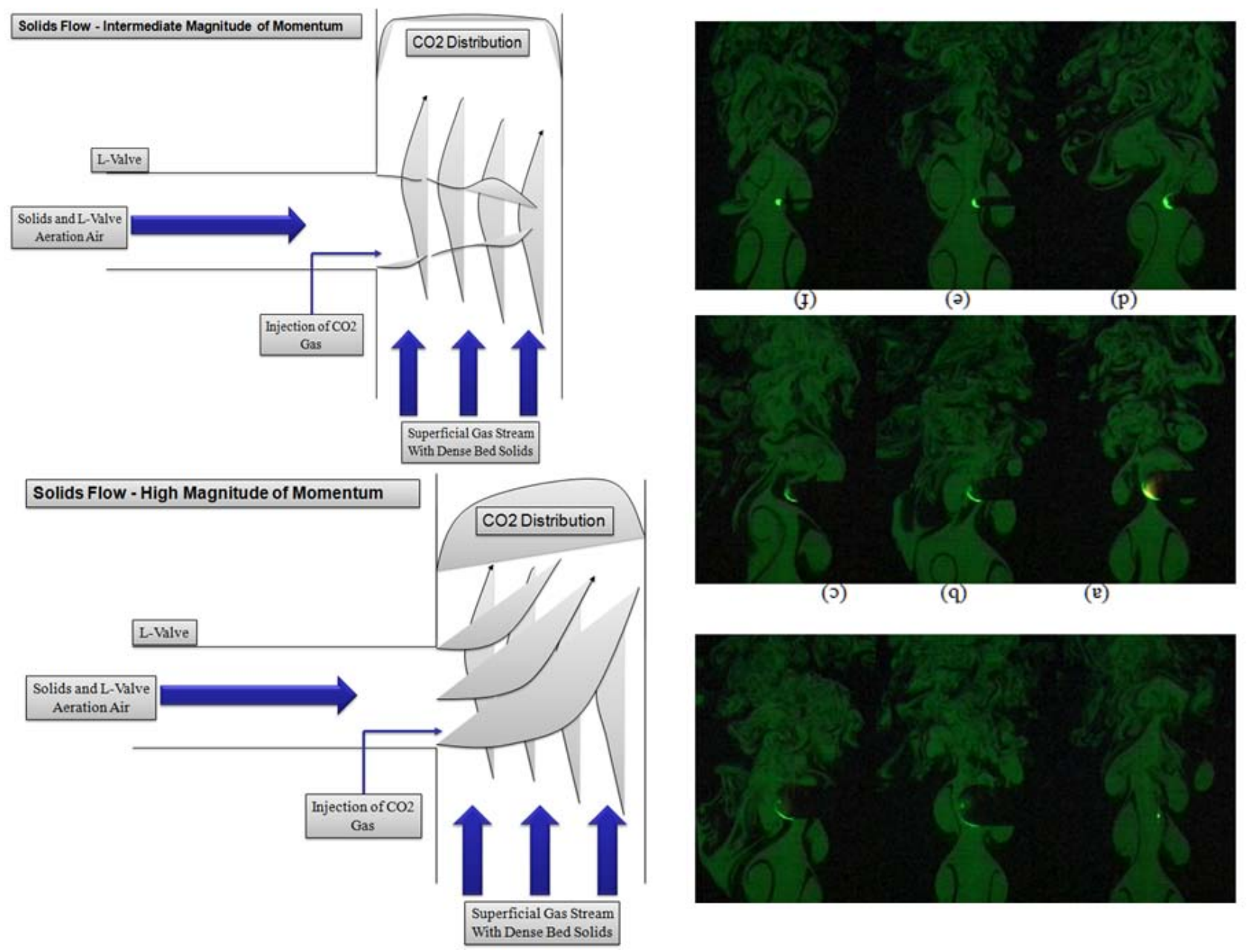

Fig 5.7 Scenario/Case 2 and 3 Modulation of Turbulence as Solids penetrate flow core

- The placed larger particles in the flow field accelerate the flow development.

- The particle wakes interact with the jet vortex rings, which make the vortex rings break up, i.e., the large scale structures are destroyed earlier which leads to a turbulent flow pattern compared to the flow without placed larger particles.

- The flow patterns are even not changed by the placed small particles. 
- The flow over the small particles still yields to Strokes flow and no oscillating and shedding wakes behind the particles. Thus the jet flow pattern is very similar with that of particle-free jet such as in scenario 1 .

As a conclusion, the presence of particles with low Reynolds number tends to suppress the turbulence of the carrier fluid. Particles with high Reynolds number as in this case enhance the turbulence most likely due to vortex shedding. Thus in the range of experimental conditions of this study $\left(\mathrm{U}_{\mathrm{g}}=5.486 \mathrm{~m} / \mathrm{s}\right.$ to $7.620 \mathrm{~m} / \mathrm{s}$ and $\mathrm{G}_{\mathrm{s}}=$ $5103 \mathrm{~kg} / \mathrm{hr}$ to $41050 \mathrm{~kg} / \mathrm{hr}$ ) the skewed and kurtotic tracer gas distribution was found to decrease from a low solids circulation rate to a high solids circulation rate since the amount of vortex shedding increased with the increase in the solids volume fraction leading to a increased turbulence. 


\section{Chapter VI}

\section{Statistical Analysis of the Gas Tracer Results}

\subsection{Introduction}

In this chapter, the experimental results obtained from the gas tracer experiments are analyzed using a statistical technique called Analysis of Variance (ANOVA). The objective of this analysis is to answer some key questions regarding the data/results obtained. In other words, our aim is to verify or prove some important hypothesis inferred from the gas tracer results. This statistical hypothesis we test is called the Null Hypothesis symbolized as $\mathrm{H}_{0}$. Its function is to specify the values of a particular parameter (the mean $\mu$, for instance) in the different treatment populations or data groups ( $\mu_{1}, \mu_{2}, \mu_{3}$, and so on). The Null Hypothesis gives the parameters the same value for all the data groups or treatment populations:

\section{$\mathrm{H}_{0}: \mu_{1}=\mu_{2}=\mu_{3}=$ and so on.}

This is tantamount of saying that no treatment effects are present in the population. The other hypothesis we propose is the Alternative Hypothesis symbolized as $\mathrm{H}_{1}$. The alternative hypothesis specifies values for the parameters of the data groups or treatment populations that are incompatible with the null hypothesis. Usually the alternative hypothesis states that the values of the parameter in the different treatment populations or data groups are not all equal. Specifically,

\section{$H_{1}$ : not all $\mu$ 's are equal.}


A decision to reject $\mathrm{H}_{0}$ implies an acceptance of $\mathrm{H}_{1}$, which implies that the means of each treatment population or data group are not close to each other and are quite distinct. On the other hand, if the means of each data group or treatment populations are quite close each other then in that case the null hypothesis is accepted and not rejected.

The reason this analysis is called ANOVA rather than multi-group means analysis (or something like that) is because it compares group means by analyzing comparisons of variance estimates. Consider:

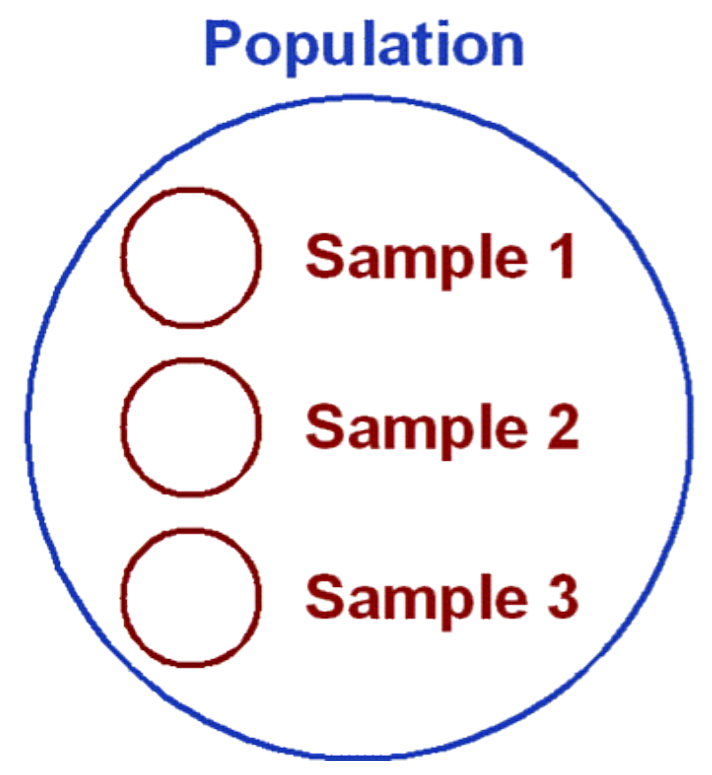

Fig.6.1. Random Samples being selected from a Global Set

We draw three random samples from a global set as shown in figure 6.1. It is quite possible that the means of each of these three samples might differ from each other. There are two possible reasons for the means to differ;

1. Group Membership (i.e., the treatment effect).

2. Differences not due to group membership (i.e., chance or sampling error). 
ANOVA is based on the fact that two independent estimates of the population variance can be obtained from the sample data. A ratio is formed for the two estimates, where: the between groups estimate is sensitive to the treatment effect and error combined and the within groups estimate is sensitive to just the error.

When the Null Hypothesis is true (in this case $\mathrm{H}_{0}: \mu_{1}=\mu_{2}=\mu_{3}$ ) the two variance estimates should be equal. That is, since the null assumes no treatment effect, both variance estimates reflect error and their ratio will equal 1 . To the extent that this ratio is larger than 1 , it suggests a treatment effect (i.e., differences between the data groups). In other words, experimental error is reflected in two ways in the results of an experiment; as differences among subjects, given the same treatment and as differences among groups of subjects given different treatments. This can be expressed as a ratio:

$$
\frac{\text { differences among different groups of subjects }}{\text { differences among subjects in the same groups }}
$$

When the null hypothesis is true, you can think of this ratio as contrasting two sets of differences that each reflects the presence of experimental error;

$$
\frac{\text { experimental error }}{\text { experimental error }} \approx 1
$$

If this experiment is repeated a large number of times on new samples of subjects drawn from the same population, the expected average value of this ratio would be about 1.0. Now if the null hypothesis is false, there is an additional 
component in the numerator, one that reflects the treatment effects. Symbolically the ratio becomes;

$$
\frac{\text { treatment effects }+ \text { experimental error }}{\text { experimental error }}
$$

If this experiment is repeated a large number of times, the expected average value of this ratio will be greater than 1.0. The ratio of these two variance estimates is distributed as an F-Distribution when the Null Hypothesis is true (Keppel and Wickens "Design and Analysis: A Researchers Handbook" Prentice Hall Publication, 2004.

\subsection{The F-Distribution}

The $\mathrm{F}$-distribution is an asymmetric distribution that has a minimum value of 0 , but no maximum value. The curve reaches a peak not far to the right of 0 , and then gradually approaches the horizontal axis the larger the $\mathrm{F}$ value is. The $\mathrm{F}$ distribution approaches, but never quite touches the horizontal axis. The Fdistribution is a ratio having two degrees of freedom, $d_{1}$ for the numerator and $d_{2}$ for the denominator. For each combination of these degrees of freedom there is a different $\mathrm{F}$-distribution. The $\mathrm{F}$-distribution curve is most spread out when the degrees of freedom are small. As the degrees of freedom increase the F-distribution curve is less dispersed.

Figure 6.2 shows the shape of the $\mathrm{F}$-distribution. The $\mathrm{F}$ value is on the horizontal axis with the probability for each $\mathrm{F}$ value being represented by the vertical axis. The shaded area in the diagram represents the level of significance a shown in the table. 
There is a different F-Distribution for each combination of the degrees of freedom of the numerator and denominator. Since there are so many FDistributions, the $\mathrm{F}$ tables are organized somewhat differently than the tables for the other distributions. The three tables that are presented in the appendix are organized by the level of significance.

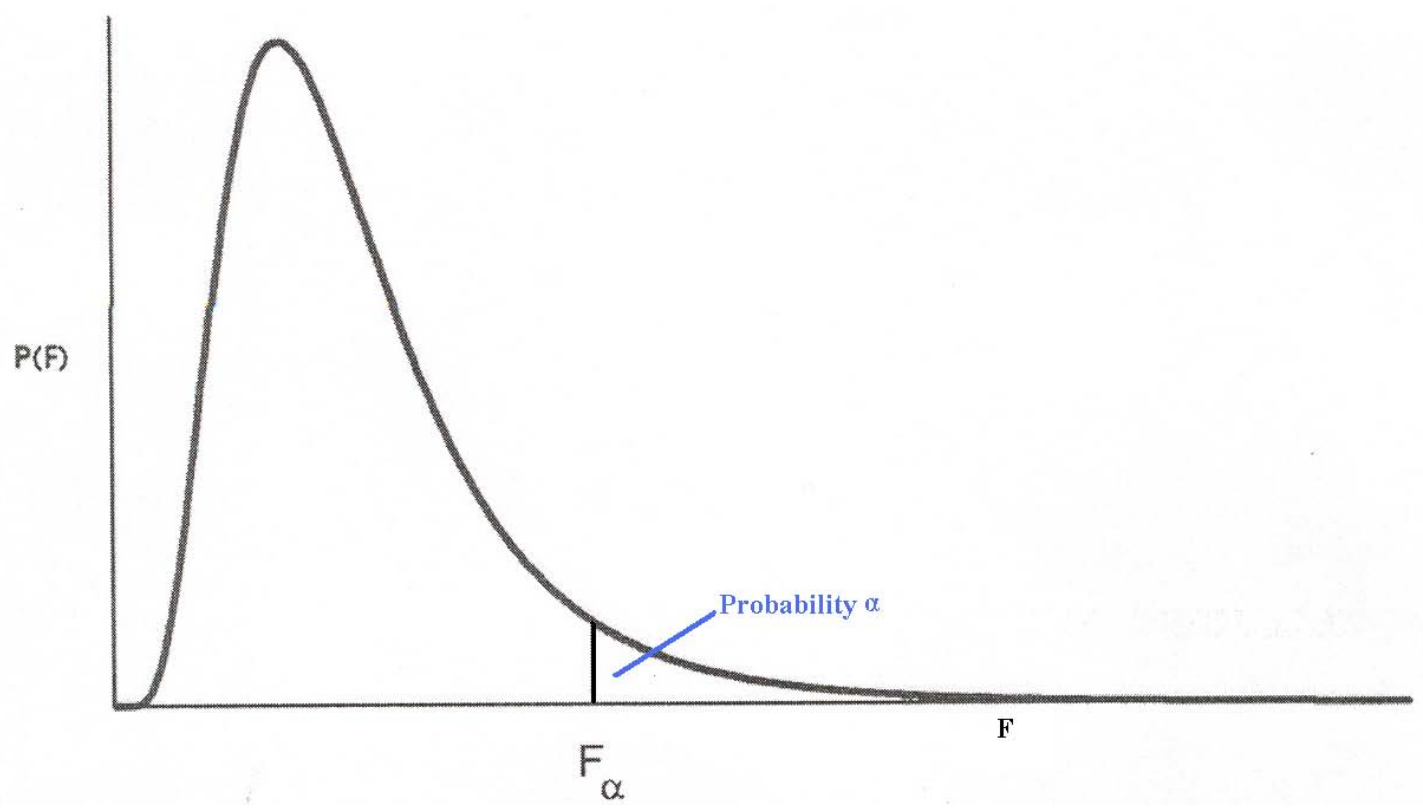

Fig.6.2.The F - Distribution

The first table gives the $\mathrm{F}$ values for that are associated with $\alpha=0.10$ of the area in the right tail of the distribution. The second table gives the $\mathrm{F}$ values for $\alpha=0.05$ of the area in the right tail and the third table gives the $\mathrm{F}$ values for $\alpha=0.01$ level of significance. In each of these tables, the $\mathrm{F}$ values are given for various combinations of degrees of freedom. In order to use the F tables, first select the level of significance to be used and then determine the appropriate combination 
of the degrees of freedom. In this thesis, the significance level was determined from the confidence interval calculations. The confidence interval calculated in Chapter IV was $95 \%$. Thus a significance of $\alpha=0.05$ was selected for this thesis. As observed from figure $6.2, \mathrm{~F}$ is positively skewed.

\subsection{Terminology}

6.3.1 Since we are talking about the Analysis of Variance, what is variance? The variance is given as

$$
\text { variance }=\frac{\text { sum of sqaured deviations from the mean }}{\text { degrees of freedom }}
$$

Thus it is essentially an average or the mean of the squared deviations about the mean.

6.3.2 Degrees of Freedom - is defined as the number of ways that the deviations are able to vary from each other. It is given as

$$
d f=(\text { number of independent variables })-(\text { number of population estimates })
$$

The degrees of freedom are different for each source of variability. These are given as

- Total Degrees of Freedom: $\mathrm{N}-1$; where $\mathrm{N}$ is the total number of values in all groups

- Within Degrees of Freedom: $d f_{\text {within }}=N-K$; where $\mathrm{K}$ is the number of categories or groups. $\mathrm{N}$ is still the total number of values in all groups

- Between Degrees of Freedom: $d f_{\text {between }}=K-1$ 


\subsubsection{Sums of Squares:}

Before proceeding ahead with calculating the sum of squares, it is important to be able to calculate the variance as a measure of the variability. With ANOVA or analysis of variance, we compute a ratio of variances: between to within variance. Recall that variance is the average square deviation of scores about the mean. We will compute the same value here, but as the definition suggests, it will be called the "mean square" for the computations.

So to calculate the variance, recall that to compute the variance we first find the sum of the square deviations, and then divide by the sample size ( $\mathrm{n}-1$ or degrees of freedom for a sample).

$$
s^{2}=\frac{\sum X^{2}-\frac{\left(\sum X\right)^{2}}{N}}{N-1}
$$

Where the numerator is the sum of squares and the denominator is the degrees of freedom. To compute the Mean Square (Variance) in order to form the F-Ratio, the same logic will be followed. Compute the sums of squares and divide by degrees of freedom. The basic logic is to find the variance for our between factor and dividing that by the variance for the within factor. These two variances will be computed by finding each sum of squares and dividing those sums of squares by their respective degrees of freedom.

The same basic formula for sums of squares will be used with variance while the between variance and within variance will be used to compute the $\mathrm{F}$ ratio. For completeness the sums of squares total (all values) will also be computed. 


\subsubsection{Total Sums of Squares: is given as}

$$
S S_{T O T}=\sum X_{T O T}^{2}-\frac{\left(\sum X_{T O T}\right)^{2}}{N_{T O T}}
$$

The subscript (TOT) stands for total. It indicates that you perform the operation for all values in your distribution (all subjects in all groups) over the total number of values in the distribution.

6.3.3.2 Within Sum of Squares: is given as

$$
S S_{\text {WITHIN }}=\left[\sum X_{1}^{2}-\frac{\left(\sum X_{1}\right)^{2}}{N_{1}}\right]+\left[\sum X_{2}^{2}-\frac{\left(\sum X_{2}\right)^{2}}{N_{2}}\right]+\cdots+\left[\sum X_{k}^{2}-\frac{\left(\sum X_{k}\right)^{2}}{N_{k}}\right]
$$

As we can see from the equation above that each segment is the same formula for sums of squares we used in the formula for variance and for the total sums of squares above. What is different here is that you consider each group separately. So, the first segment with the subscript "1" means you compute the sum of squares for the first data group. Group two is labeled with a "2", but notice that after that we have group " $\mathrm{k}$ " instead of a number. This notation indicates that you continue to find the sums of squares as you did for the first two groups for however many groups you have in the problem. So, "k" could be the third group, or if you have four groups then you would do the same sums of squares computation for the third and fourth group.

6.3.3.3 Between Sums of Squares: is given as

$$
S S_{\text {BETWEEN }}=\left[\frac{\left(\sum X_{1}\right)^{2}}{n_{1}}\right]+\left[\frac{\left(\sum X_{2}\right)^{2}}{n_{2}}\right]+\cdots+\left[\frac{\left(\sum X_{k}\right)^{2}}{n_{k}}\right]-\left[\frac{\left(\sum X_{T O T}\right)^{2}}{N_{T O T}}\right]
$$


We have the same "k" notation here. Again you perform the same operation for each separate data group in your problem. However, with this formula once we compute the value for each group we must subtract an operation at the final step. This operation is half the sums of squares we computed for the sums of squares total.

6.3.4 Mean Square: Now we divide each Sums of Squares by their respective Degrees of Freedom to get the Mean Squares.

6.3.4.1 Between Mean Square: is given as

$$
M S_{\text {Between }}=\frac{S S_{\text {Between }}}{d f_{\text {Between }}}
$$

6.3.4.2 Within Mean Square: is given as

$$
M S_{\text {Within }}=\frac{S S_{\text {Within }}}{d f_{\text {Within }}}
$$

6.3.5 F-Ratio: the final step is to divide the Between Variance by the Within Variance to see if the effect (Between) is large compared to the error (Within).

$$
F=\frac{M S_{\text {Between }}}{M S_{\text {Within }}}
$$

\subsubsection{Evaluating the F-Ratio}

The F-Ratio for the treatment effects brought into question the null hypothesis, there is however the possibility that it only reflected chance factors or chance variation. To overcome this problem we use a procedure known as the Null-Hypothesis Significance Testing, to assess the FRatio.

\subsection{Testing the Null Hypothesis}


Let us specify the null $\left(\mathrm{H}_{0}\right)$ and alternative $\left(\mathrm{H}_{1}\right)$ hypotheses, the two hypotheses are;

$$
\begin{aligned}
& H_{0}: \text { all } \mu_{j}^{\prime} s \text { are equal } \\
& H_{1} \text { : not all } \mu_{j}^{\prime} \text { s are equal }
\end{aligned}
$$

The null hypothesis will test states that the means of the treatment populations are equal. This particular null hypothesis is selected because it is usually the only hypothesis that can be stated exactly. There is no ambiguity in the assertion that the population means are equal. The alternative hypothesis is a mutually exclusive statement, which generally asserts only that some of the population treatment means are not equal - that is, that some treatment effects are present. This hypothesis however is an inexact statement. Nothing is said about the actual differences that are in the population.

Suppose we have conducted an experiment and have computed the value of the F-Ratio. We have to decide whether it came from the F-Distribution. Because we are evaluating the null hypothesis we focus on the F-Distribution. Although some values of $\mathrm{F}$ are less likely to occur than others, it is still possible theoretically to obtain any value of $\mathrm{F}$ in an experiment when the null hypothesis is true. From one point of view this situation does not bode well for the experimentalists. This is because if any value of $\mathrm{F}$ may be the result of chance factors, and then we can never be absolutely certain that the $\mathrm{F}$ we observe in an experiment did not come from the F-Distribution. However, taking this position will make it impossible to conduct any experiments in the real life and find out things about the world. Hence it is 
imperative that we risk making a mistake by rejecting the null hypothesis when it is true; otherwise we could never reject it and never learn anything

Suppose we imagine a vertical dividing line for any F-Distribution as shown in figure 6.2 , where the values of $\mathrm{F}$ falling above the line are considered to be "unlikely" and the values of $\mathrm{F}$ falling below the line are considered to be "likely". We can then determine if whether an observed $\mathrm{F}$ falls above or below this arbitrary dividing line. When the $\mathrm{F}$ value fell above the line, we conclude that the obtained $\mathrm{F}$ value is incompatible with the null hypothesis. We then reject the $\mathrm{H}_{0}$ and conclude that the alternative hypothesis is true. When the $\mathrm{F}$ value fell below the line, however, we conclude that the observed $\mathrm{F}$ value is compatible with the null hypothesis. Under these circumstances, we can safely say that the $\mathrm{H}_{0}$ holds true. $\mathrm{By}$ following this set of rules, we can conclude that an independent variable was effective whenever an $\mathrm{F}$ is obtained that falls within the region of incompatibility.

\subsubsection{Decision Rules}

The problem is to find a way to objectively define the regions of compatibility and incompatibility. When the null hypothesis is true, we can determine the sampling distribution of F. Suppose we find a point on this distribution beyond which the probability of finding $\mathrm{F}$ is very small - the probability is represented by the proportion of the total area under the curve that appears beyond this point. We adopt the convention that the values of $\mathrm{F}$ falling above the dividing line are incompatible with the null hypothesis. This decision rule, then, is to reject the null 
hypothesis when the observed $\mathrm{F}$ value falls within this region of incompatibility. One risk being, that we will occasionally make the wrong decision.

Suppose we begin to enlarge the region of incompatibility by moving the critical point of transition to the left - towards the larger portion of the curve. We as we increase the size of the region, we also increase the chance of observing values from it. That is increasing the region of incompatibility results in the inclusion of F's that are increasingly more compatible with the null hypothesis. Thus the dilemma is how big should this region of incompatibility? We can take any probability as long as we make the decision before the start of the experiment. In practice, however, there is a fairly common agreement on a probability of $\alpha=0.05$ to define the region of incompatibility for the F-Distribution (3). This probability is called the Significance Level of the test. This significance level a defines a

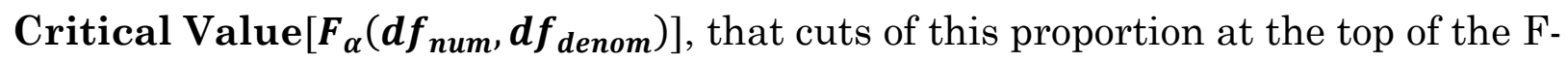
Distribution. For example we have calculated the value $F_{\text {calculated }}$ from the data. If the $F_{\text {calculated }}$ value falls within the region of incompatibility determined by $\left[F_{\alpha}\right]$, then the null hypothesis is rejected and the alternative hypothesis is accepted. If the $F_{\text {calculated }}$ value falls within the region of compatibility then the null hypothesis is not rejected. Symbolically, this rule is;

$\left\{\begin{array}{l}\text { If } F_{\text {calculated }} \geq F_{\alpha}\left(d f_{\text {num }}, d f_{\text {denom }}\right), \text { then reject } H_{0} \text { and retain } H_{1} \\ \text { If } F_{\text {calculated }}<F_{\alpha}\left(d f_{\text {num }}, d f_{\text {denom }}\right), \text { then retain } H_{0} \text { and reject } H_{1}\end{array}\right\}$

Once we have calculated the $\mathrm{F}$ from the equation above, to decide if the dependent variable is significantly influenced by the independent variable, we compare the calculated $\mathrm{F}$ value with a $\mathrm{F}_{\mathrm{a}}$ value determined from the $\mathrm{F}$-distribution 
tables. If $\mathrm{F}_{\text {calc }}$ is greater than $\mathrm{F}_{\alpha}$, then the dependent variable is significantly influenced by the independent variable. If the $\mathrm{F}_{\text {calc }}$ is less than $\mathrm{F}_{\mathrm{a}}$, then the dependent variable is not significantly influenced by the independent variable. This decision making rule can also be expressed as two ratio given below

$$
\begin{aligned}
& \frac{F_{\text {observed }}}{F_{\alpha}}>1---(1) \\
& \frac{F_{\text {observed }}}{F_{\alpha}} \leq 1---(2)
\end{aligned}
$$

For if ratio (1) holds true then the dependent variable is significantly influenced by the independent variable and if ratio (2) holds true then the dependent variable is not significantly influenced by the independent variable.

This chapter discusses the effects of different CFB operational variables such as solids circulation rate, superficial gas velocity, radial and axial elevations, azimuthal direction. A list of variables has been provided in table 6.2. Using the Analysis of Variance we are interested in answering the following questions. 1) Is there significant dependence of the $\mathrm{CO} 2$ concentration on the independent variables listed in table 6.2 for the entire dense entry region? 2).Is there a significant dependence of the $\mathrm{CO} 2$ concentration on the variation between the operating conditions at each axial elevation? 3).Is there is a significance dependence of the CO2 concentration on the axial elevation?

Answering all these questions will provide the design engineers with important information which will assist them in designing better gasifiers. This is because the hydrodynamics of the dense entry region has a significant influence on 
the hydrodynamics of the fully developed and dilute regions of a circulating fluidized bed riser.

\subsection{Checking for Significance}

In this section we are seeing if there is a significant dependence of the $\mathrm{CO} 2$ concentration on the variation (levels) of each independent variable for the entire dense entry region. Table 6.2 gives us the levels of each independent variable. As you can see from table 6.2, there three levels of $U_{g}$, five levels of $M_{s}$, seven levels of r/R ( $\left.\mathrm{R}^{\circ}\right)$, four levels of Axial Elevation (Ъ), and two levels of Azimuthal Position (s).

\begin{tabular}{|c|c|c|}
\hline $\begin{array}{c}\text { Independent } \\
\text { Variable }\end{array}$ & Description of Variable & Levels \\
\hline $\mathbf{U}_{\mathrm{g}}$ & Superficial Gas Velocity & 3 \\
\hline $\mathbf{M}_{\mathrm{s}}$ & Solids Circulation Rate & 3 \\
\hline $\mathbf{r}$ & Radial Position Normalized with Riser Radius & 7 \\
\hline 3 & Axial Elevation & 4 \\
\hline S & Azimuthal Position & 2 \\
\hline
\end{tabular}

Table.6.2. Levels of the Independent Variables

Table 6.3 to Table 6.7 gives the Analysis of Variance results for checking the significant dependency of the tracer gas concentration on the each individual independent variable listed in Table 6.2.

\begin{tabular}{|l|c|c|c|c|c|c|}
\hline \multicolumn{7}{|c|}{ ANOVA Table for $U_{\mathrm{g}}$} \\
\hline Source of Variation & SS & df & MS & F & F $^{*}$ & $\%\left(F-F^{*}\right)$ \\
\hline
\end{tabular}




\begin{tabular}{|c|c|c|c|c|c|c|}
\hline Rows (Tracer Conc.) & 161791680.000 & 55 & 2941666.909 & 104.952 & 19.478 & 438.825 \\
\hline Columns(Ug Levels) & 56057.238 & 2 & 28028.619 & 25.017 & & \\
\hline Error & 374209.429 & 334 & 1120.388 & & & \\
\hline & & & & & & \\
\hline Total & 16221946.667 & 391 & & & & \\
\hline
\end{tabular}

Table 6.3 Dependence on $\mathrm{U}_{\mathrm{g}}$

\begin{tabular}{|c|c|c|c|c|c|c|}
\hline \multicolumn{7}{|c|}{ ANOVA Table for M $_{\text {s }}$} \\
\hline Source of Variation & SS & df & MS & F & $F^{*}$ & $\%(F-F *)$ \\
\hline Rows (Tracer Conc.) & 218726942.629 & 55 & 3976853.502 & 118.238 & 19.478 & 507.033 \\
\hline Columns(Ms Levels) & 67268.686 & 2 & 33634.343 & 257.365 & & \\
\hline Error & 197627.680 & 334 & 130.687 & & & \\
\hline & & & & & & \\
\hline Total & 218991838.994 & 391 & & & & \\
\hline
\end{tabular}

Table 6.4 Dependence on $\mathrm{M}_{\mathrm{s}}$

\begin{tabular}{|c|c|c|c|c|c|c|}
\hline \multicolumn{7}{|c|}{ ANOVA Table for $\mathbf{R}^{*}$} \\
\hline Source of Variation & SS & df & MS & $\mathbf{F}$ & $\mathbf{F}^{*}$ & $\%(F-F *)$ \\
\hline Rows (Tracer Conc.) & 77274788.114 & 55.000 & 1404996.148 & 20.886 & 3.000 & 596.211 \\
\hline Columns( $\mathbf{R}^{*}$ Levels $)$ & 403612.114 & 6.000 & 67268.686 & & & \\
\hline Error & 346336.315 & 330.000 & 1049.501 & & & \\
\hline Total & 78024736.544 & 391.000 & & & & \\
\hline
\end{tabular}

Table 6.5 Dependence on $\mathrm{R}^{*}$

\begin{tabular}{|c|c|c|c|c|c|c|}
\hline \multicolumn{7}{|c|}{ ANOVA Table for Axial Elevation (马) } \\
\hline Source of Variation & SS & $\mathrm{df}$ & MS & $\mathrm{F}$ & $\mathrm{F}^{*}$ & $\%\left(F-F^{*}\right)$ \\
\hline
\end{tabular}




\begin{tabular}{|c|c|c|c|c|c|c|}
\hline Rows (Tracer Conc.) & 138832670.171 & 97.000 & 1431264.641 & 21.277 & 8.567 & 148.358 \\
\hline Columns(ろ Levels) & 201806.057 & 3.000 & 67268.686 & & & \\
\hline Error & 311221.926 & 291.000 & 1069.491 & & & \\
\hline & & & & & & \\
\hline Total & 139345698.154 & 391.000 & & & & \\
\hline
\end{tabular}

Table 6.6 Dependence on Axial Elevation (马)

\begin{tabular}{|c|c|c|c|c|c|c|}
\hline \multicolumn{7}{|c|}{ ANOVA Table for Azimuthal Position (s) } \\
\hline Source of Variation & SS & df & MS & F & $\mathrm{F}^{*}$ & $\%\left(\mathrm{~F}_{-} \mathrm{F}^{*}\right)$ \\
\hline Rows (Tracer Conc.) & 261948434.286 & 195.000 & 1343326.304 & 18.154 & 10.210 & 77.807 \\
\hline Columns(s Levels) & 73996.554 & 1.000 & 73996.554 & & & \\
\hline Error & 192201.794 & 195.000 & 986.650 & & & \\
\hline & & & & & & \\
\hline Total & 262214631.634 & 391.000 & & & & \\
\hline
\end{tabular}

Table 6.7 Dependence on Azimuthal Position (s)

In all the tables the F statistic calculated in the "F" column is the calculated F value. The $\mathrm{F}^{*}$ statistic is the value determined from the $\mathrm{F}$ distribution tables in appendix V.1. To determine if the independent variable significantly influences the dependent variable $\left(\mathrm{CO}_{2}\right.$ concentration) the calculated $\mathrm{F}$-statistic value should be higher than the $\mathrm{F}^{*}$ statistic. If the calculated $\mathrm{F}$-statistic is less than the $\mathrm{F}^{*}$ statistic then the dependent variable $\left(\mathrm{CO}_{2}\right.$ concentration) is not significantly influenced by the independent variables. The $\mathrm{F}^{*}$ statistic is determined from the F-distribution table based on the degrees of freedom of the independent variable and the number of observations made for each level of the independent variable. A percentage error 
is determined between the two statistics, i.e. the calculated F-statistic and the theoretical $\mathrm{F}^{*}$-statistic. If the $\mathrm{F}^{*}$-statistic is the same for several independent variables or analyses, a comparative study is conducted. In this study, we analyze the percentage error values. A higher error signifies a higher magnitude of dependency of the dependent variable $\left(\mathrm{CO}_{2}\right.$ concentration) on the independent variables. In other words the larger the error, the higher the degree of significant influence of the independent variable on the dependent variable. This is shown in figure 6.3 below.

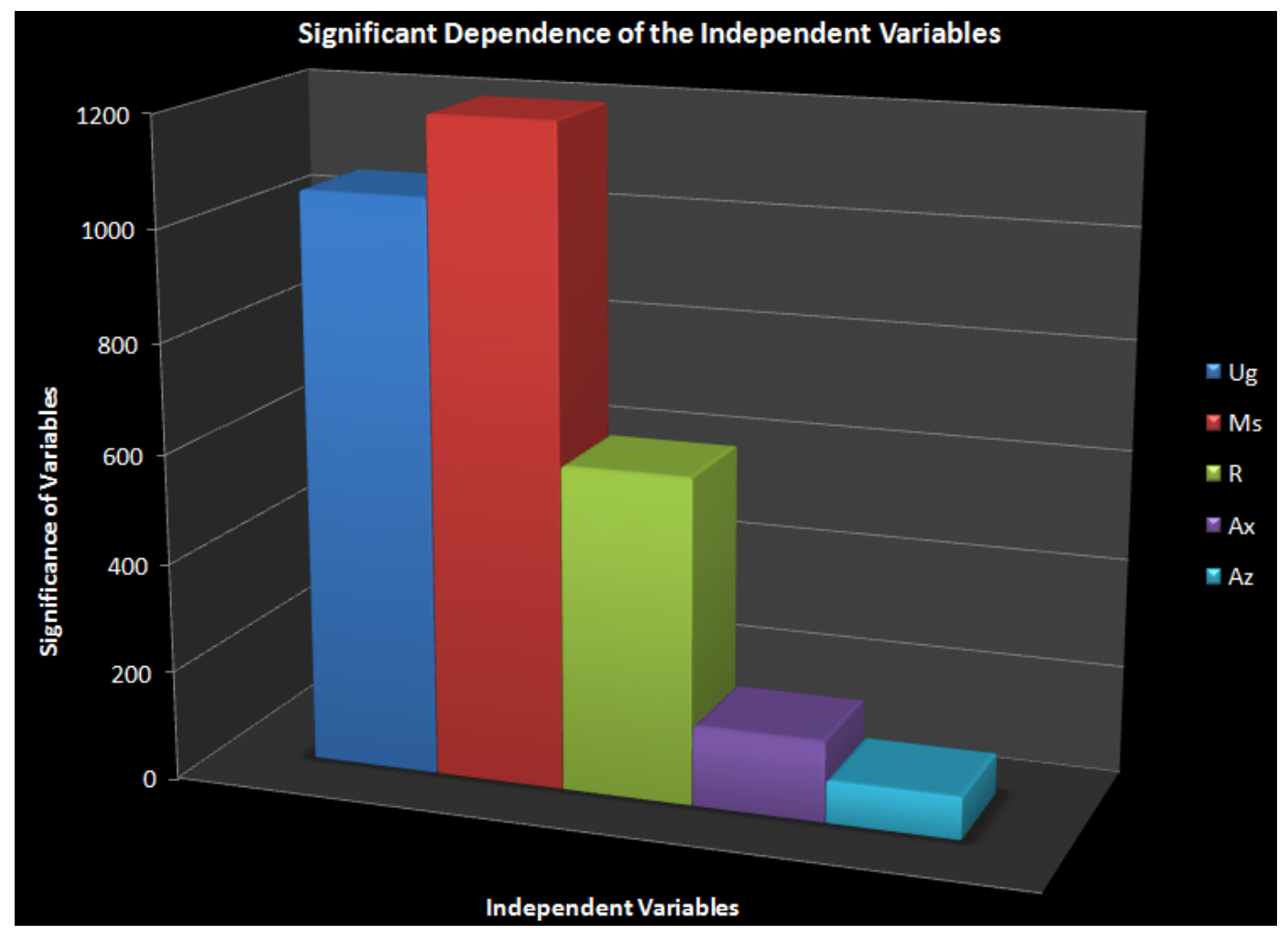

Fig.6.3 Magnitudes of significant influences of the Primary Independent Variables As seen from figure 6.3, from a comparative analysis of the percentage error between $U_{g}, M_{s}$ and $R$, we can rank the three in ascending order of influence given as; $\mathrm{R}<\mathrm{U}_{\mathrm{g}}<\mathrm{M}_{\mathrm{s}}$. Since the other two variables, i.e. the axial elevation and azimuthal 
position each had varying degrees of freedom, they each had different $\mathrm{F}^{*}$ statistic values. This prevented any comparative analysis.

Tables 6.8 to 6.17 show the ANOVA results which check for the dependence of the tracer gas concentration on the variation within the operating conditions for all axial and azimuthal positions.

\begin{tabular}{|c|c|c|c|c|c|c|}
\hline \multicolumn{7}{|c|}{ Variation Between Operating Conditions (OCs) - 2.5 Feet East West } \\
\hline \multicolumn{7}{|c|}{ ANOVA Results } \\
\hline Source of Variation & SS & df & MS & F & $F^{*}$ & $\%($ F-F*) \\
\hline Rows (Tracer Conc.) & 4622619.429 & 6.000 & 770436.571 & 11.453 & 4.280 & 167.596 \\
\hline Columns(OCs Levels) & 403612.114 & 6.000 & 67268.686 & & & \\
\hline Error & 40544.511 & 36.000 & 8216.598 & & & \\
\hline & & & & & & \\
\hline Total & 5066776.054 & 48.000 & & & & \\
\hline
\end{tabular}

Table 6.8 Dependence on the variation within operating conditions at 2.5 feet (eastwest)

\begin{tabular}{|c|c|c|c|c|c|c|}
\hline \multicolumn{7}{|c|}{ Variation Between Operating Conditions - 2.5 Feet North South } \\
\hline \multicolumn{7}{|c|}{ ANOVA } \\
\hline Source of Variation & SS & df & MS & F & F* & $\%($ F-F*) \\
\hline Rows (Tracer Conc.) & 5200446.857 & 6.000 & 866741.143 & 11.625 & 4.280 & 171.620 \\
\hline Columns(OCs Levels) & 447336.760 & 6.000 & 74556.127 & & & \\
\hline Error & 244590.311 & 36.000 & 5402.049 & & & \\
\hline & & & & & & \\
\hline Total & 5892373.928 & 48.000 & & & & \\
\hline
\end{tabular}


Table 6.9 Dependence on the variation within operating conditions at 2.5 feet (north-south)

\begin{tabular}{|c|c|c|c|c|c|c|}
\hline \multicolumn{7}{|c|}{ Variation Between Operating Conditions - 3.5 Feet East West } \\
\hline \multicolumn{7}{|c|}{ ANOVA } \\
\hline Source of Variation & SS & df & MS & F & $F^{*}$ & $\%($ F-F*) \\
\hline Rows (Tracer Conc.) & 3852182.857 & 6.000 & 642030.476 & 8.810 & 4.280 & 106.843 \\
\hline Columns(OCs Levels) & 437246.457 & 6.000 & 72874.410 & & & \\
\hline Error & 115739.503 & 36.000 & 7454.730 & & & \\
\hline & & & & & & \\
\hline Total & 4405168.817 & 48.000 & & & & \\
\hline
\end{tabular}

Table 6.10 Dependence on the variation within operating conditions at 3.5 feet (east-west)

\begin{tabular}{|c|c|c|c|c|c|c|}
\hline \multirow{2}{*}{\multicolumn{7}{|c|}{$\begin{array}{l}\text { Variation Between Operating Conditions - 3.5 Feet North South } \\
\text { ANOVA }\end{array}$}} \\
\hline & & & & & & \\
\hline Source of Variation & SS & df & MS & $\mathbf{F}$ & $\mathbf{F}^{*}$ & $\%\left(\mathrm{~F}-\mathrm{F}^{*}\right)$ \\
\hline Rows (Tracer Conc.) & 3599864.880 & 6.000 & 599977.480 & 7.783 & 4.280 & 81.842 \\
\hline Columns(OCs Levels) & 462539.483 & 6.000 & 77089.914 & & & \\
\hline Error & 266547.539 & 36.000 & 7404.098 & & & \\
\hline Total & 4328951.902 & 48.000 & & & & \\
\hline
\end{tabular}

Table 6.11 Dependence on the variation within operating conditions at 3.5 feet (north-south)

\section{Variation Between Operating Conditions - 6.5 Feet East West}




\begin{tabular}{|c|c|c|c|c|c|c|}
\hline \multicolumn{7}{|c|}{ ANOVA } \\
\hline Source of Variation & SS & df & MS & F & F $^{*}$ & \%(F-F*) \\
\hline Rows (Tracer Conc.) & 3081746.286 & 6.000 & 513624.381 & 6.545 & 4.280 & 52.912 \\
\hline Columns(OCs Levels) & 470880.800 & 6.000 & 78480.133 & & & \\
\hline Error & 342650.975 & 36.000 & 9518.083 & & & \\
\hline & & & & & & \\
\hline Total & 3895278.061 & 48.000 & & & & \\
\hline
\end{tabular}

Table 6.12 Dependence on the variation within operating conditions at 6.5 feet (east-west)

\begin{tabular}{|c|c|c|c|c|c|c|}
\hline \multicolumn{7}{|c|}{ Variation Between Operating Conditions - 6.5 Feet North South } \\
\hline Source of Variation & SS & df & MS & F & F $^{*}$ & \%(F-F*) \\
\hline Rows (Tracer Conc.) & 2786669.079 & 6.000 & 464444.846 & 6.206 & 4.280 & 45.003 \\
\hline Columns(OCs Levels) & 449018.477 & 6.000 & 74836.413 & & & \\
\hline Error & 167510.297 & 36.000 & 4653.064 & & & \\
\hline & & & & & & \\
\hline Total & 3403197.853 & 48.000 & & & & \\
\hline
\end{tabular}

Table 6.13 Dependence on the variation within operating conditions at 6.5 feet (north-south)

\begin{tabular}{|c|c|c|c|c|c|c|}
\hline \multicolumn{7}{|c|}{ Variation Between Operating Conditions - 13.5 Feet East West } \\
\hline \multicolumn{7}{|c|}{ ANOVA } \\
\hline Source of Variation & SS & df & MS & F & $F^{*}$ & $\%\left(F-F^{*}\right)$ \\
\hline Rows (Tracer Conc.) & 2311309.714 & 6.000 & 385218.286 & 4.581 & 4.280 & 7.039 \\
\hline
\end{tabular}




\begin{tabular}{|c|c|c|c|c|c|c|}
\hline Columns(OCs Levels) & 504516.143 & 6.000 & 84086.857 & & & \\
\hline Error & 245148.809 & 36.000 & 6809.689 & & & \\
\hline & & & & & & \\
\hline Total & 3060973.666 & 48.000 & & & & \\
\hline
\end{tabular}

Table 6.14 Dependence on the variation within operating conditions at 13.5 feet (east-west)

\begin{tabular}{|c|c|c|c|c|c|c|}
\hline \multicolumn{7}{|c|}{ Variation Between Operating Conditions - 13.5 Feet North South } \\
\hline Source of Variation & SS & $\mathrm{df}$ & MS & F & F $^{*}$ & $\%\left(F_{-} \mathrm{F}^{*}\right)$ \\
\hline Rows (Tracer Conc.) & 1835566.131 & 6.000 & 305927.522 & 4.546 & 4.280 & 6.205 \\
\hline Columns(OCs Levels) & 403813.920 & 6.000 & 67302.320 & & & \\
\hline Error & 247284.780 & 36.000 & 6869.022 & & & \\
\hline & & & & & & \\
\hline Total & 2486663.832 & 48.000 & & & & \\
\hline
\end{tabular}

Table 6.15 Dependence on the variation within operating conditions at 13.5 feet (north-south)

\begin{tabular}{|c|c|c|c|c|c|c|}
\hline \multicolumn{7}{|c|}{ Variation Between Operating Conditions - 28.5 Feet East West } \\
\hline SS & $\mathrm{df}$ & MS & F & $\mathrm{F}^{*}$ & $\%($ F-F*) \\
\hline Source of Variation & SS & & & & \\
\hline Rows (Tracer Conc.) & 2022396.000 & 6.000 & 337066.000 & 3.758 & 4.280 & 12.195 \\
\hline Columns(OCs Levels) & 538149.486 & 6.000 & 89691.581 & & & \\
\hline Error & 241994.871 & 36.000 & 6722.081 & & & \\
\hline & & & & & & \\
\hline
\end{tabular}




\begin{tabular}{|c|c|c|c|c|c|c|}
\hline Total & 2802540.356 & 48.000 & & & & \\
\hline
\end{tabular}

Table 6.16 Dependence on the variation within operating conditions at 28.5 feet (east-west)

\begin{tabular}{|c|c|c|c|c|c|c|}
\hline \multicolumn{7}{|c|}{ Variation Between Operating Conditions - 28.5 Feet North South } \\
\hline \multicolumn{7}{|c|}{ ANOVA } \\
\hline Source of Variation & SS & df & MS & $\mathbf{F}$ & $\mathbf{F}^{*}$ & $\%\left(F-F^{*}\right)$ \\
\hline Rows (Tracer Conc.) & 1835566.131 & 6.000 & 305927.522 & 3.912 & 4.280 & 8.608 \\
\hline Columns(OCs Levels) & 469266.352 & 6.000 & 78211.059 & & & \\
\hline Error & 45441.327 & 36.000 & 1262.259 & & & \\
\hline Total & 2350272.810 & 48.000 & & & & \\
\hline
\end{tabular}

Table 6.17 Dependence on the variation within operating conditions at 28.5 feet (north-south)

Again we can clearly see that the variation within the operating conditions significantly influences the tracer gas concentration. This holds true for all axial elevations and both azimuthal positions. For this analysis, the $\mathrm{F}^{*}$ statistic is constant for all cases, as the degrees of freedom is the same for all conditions. Thus the percentage error based on the difference of the calculated F-statistic and the theoretical $\mathrm{F}^{*}$-statistic gives a good indication of how the significance varies with the changes in operating conditions for each elevation and azimuthal position. Further analysis of the percentage error also reveals how the magnitude of dependence or significant influence decreases with elevation for both azimuthal positions. This is shown in figure 6.4 , 


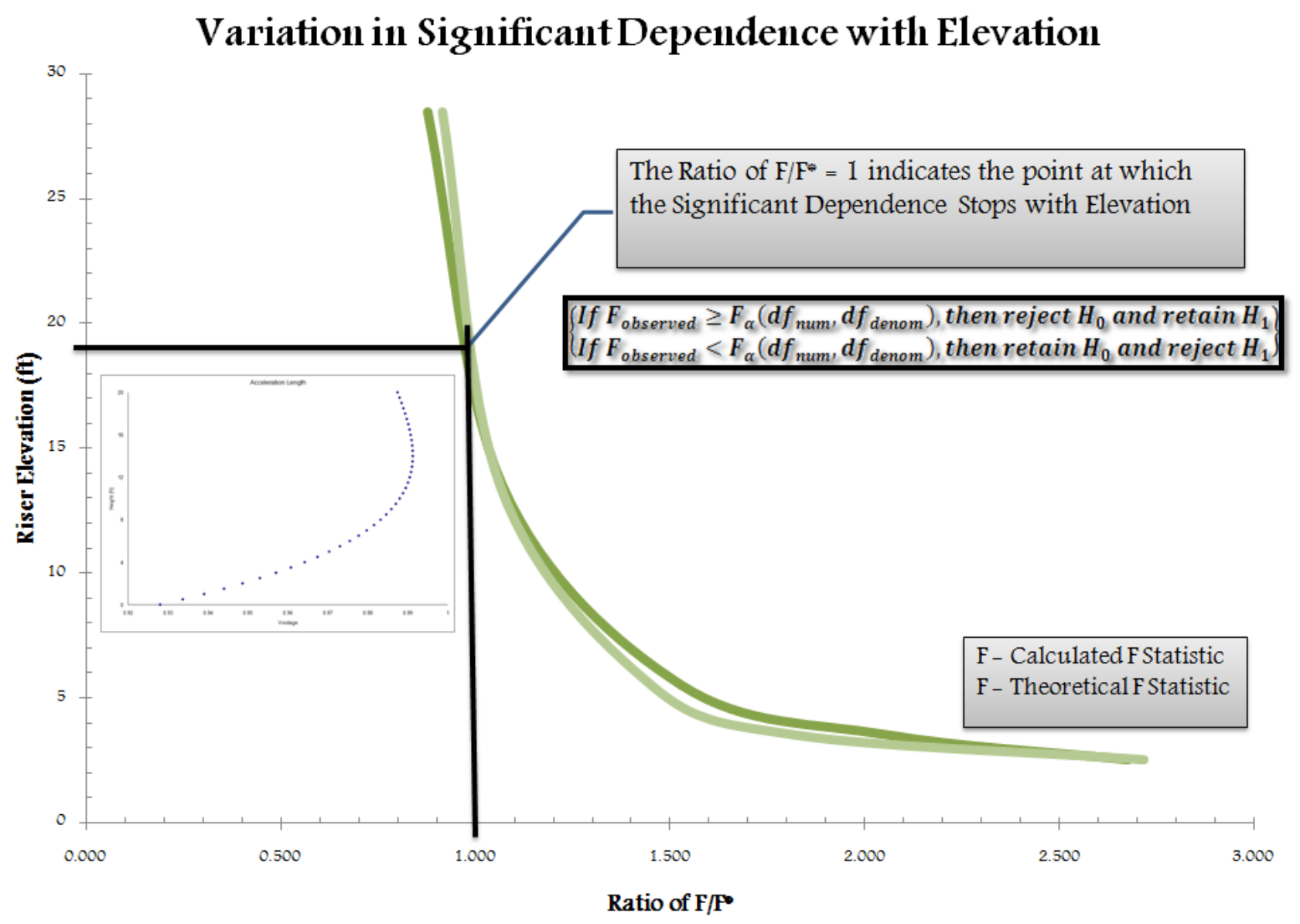

Fig.6.4 Variation in magnitude of dependence on operating conditions, the length of the acceleration region is shown in the small box diagram (Monazam, 2004).

As we can see from the figure, the magnitude of dependence decreases with elevation for both azimuthal positions. Between 13.5 feet and 28.5 feet, the percentage error shows a negligible increase and beyond 28.5 feet elevation it is impossible to say with certainty that the variation seen in the tracer gas concentrations is because of the variations in the operating conditions. This holds true for both azimuthal positions.

ANOVA Table for Significance of Axial Elevation

Source of Variation SS df MS F $\begin{array}{ll}\mathrm{F}^{*} & \%\left(\mathrm{~F}-\mathrm{F}^{*}\right)\end{array}$ 


\begin{tabular}{|c|c|c|c|c|c|c|}
\hline Rows (Tracer Conc.) & 4618767.246 & 6.000 & 769794.541 & 19.222 & 8.940 & 115.013 \\
\hline Columns(Axial Elevations) & 120141.873 & 3.000 & 40047.291 & & & \\
\hline Error & 360689.423 & 18.000 & 20038.301 & & & \\
\hline & & & & & \\
\hline Total & 5099598.541 & 27.000 & & & & \\
\hline
\end{tabular}

Table 6.18 Dependence on the variation of axial elevation in the East - West Azimuth Position

\begin{tabular}{|c|c|c|c|c|c|c|}
\hline \multicolumn{7}{|c|}{ ANOVA Table for Significance of Axial Elevation } \\
\hline Source of Variation & SS & df & MS & F & $F^{*}$ & $\%\left(F_{-} F^{*}\right)$ \\
\hline Rows (Tracer Conc.) & 3505486.400 & 6.000 & 584247.733 & 16.528 & 8.940 & 73.690 \\
\hline Columns(Axial Elevations) & 112876.855 & 3.000 & 37626.618 & & & \\
\hline Error & 288229.182 & 18.000 & 16012.732 & & & \\
\hline & & & & & & \\
\hline Total & 3906592.436 & 27.000 & & & & \\
\hline
\end{tabular}

Table 6.19 Dependence on the variation of axial elevation in the North - South Azimuth Position

Table 6.18 and table 6.19 gives the ANOVA analysis for the significant dependence or the influence of the axial elevation on the tracer gas concentration for azimuthal direction of east - west direction and north - south direction respectively. In this case, concentrations recorded for all radial positions for each axial elevation were taken for one particular operating condition. The operating condition selected was the mid-point of the factorial test matrix. We can clearly see that the variation in the axial elevation is a significantly influencing factor for both 
azimuthal positions as the calculated F-statistic is higher than the theoretical $\mathrm{F}^{*}$ statistic determined from the F-distribution table. It is also evident that effect of the variation in the Axial Elevation in the East - West Azimuthal Position has a higher magnitude of significance than the variation in Axial Elevation in the North South Azimuthal Position based on the comparative analysis of the percentage error.

\subsection{Effect Size, Power and Sample Size}

The results of this experiment are expressed most directly by the values of the sample means. In the previous sections we have described the pattern of the means and estimates of their variability, usually the standard deviations of their scores. However, an important information, a designer will like to have is an overall measure of the magnitude of the effect that incorporates all the groups at once.

\subsubsection{Descriptive Measures of Effect Size}

An effect size measure is a quality that measures the size of an effect as it exists in the population, in a way that is independent of certain details of the experiment such as the sizes of the samples used. We cannot use the F-statistic or its associated p-value as a measure of effect size because of two problems. One problem is that when there are differences among $\mu_{\mathrm{j}}$ in the population, a study that uses large samples tends to give a larger $\mathrm{F}$ value and a smaller $\mathrm{p}$ value than does a study with small samples. Thus these quantities depend on both the population and particulars of the study. 
A related problem is that a test statistic such as $\mathrm{F}$ and its associated $\mathrm{p}$ value have no population counterparts. Descriptive measures of effect size, other than the means themselves, can generally be divided into two types, those that describe the differences in the means relative to the study's variability and those that look at how much of the variability can be attributed to the treatment conditions. Each type emphasizes a different aspect of the effect, and each has its place in describing a study.

\subsubsection{Differences Relative to Variability of the Observations}

The first measure is often known as the standardized difference between the means. Consider a study where there are two groups A and B. The means of these two groups are $\overline{Y_{1}}=15, \overline{Y_{2}}=6$ and the sample size $(n=5)$. We can say that the effect of the action implemented on the two groups is to reduce the scores by $15-6=9$ points. Useful as this quantity is, it takes no account of how much variability there is in scores. Is a 9 point difference large or small relative to the extent to which performance varies from one element of the groups to another?

To obtain a measure of the effect that takes this variability into account, we divide the difference by the standard deviation of the scores:

$$
d_{12}=\frac{\overline{Y_{1}}-\overline{Y_{2}}}{s_{12}}
$$

The standard deviation $s_{12}$ in the equation above is the pooled standard deviation of the two groups. With equal sample sizes, it equals the average of the variance: 


$$
s_{12}=\sqrt{\frac{s_{1}^{2}+s_{2}^{2}}{2}}
$$

With unequal sample sizes, we must pool the sums of the squares and degrees of freedom separately, as we did when finding the mean square in a familiar situation

$$
s_{12}=\sqrt{\frac{S S_{1}+S S_{2}}{d f_{1}+d f_{2}}}
$$

In this example, the two groups contain the same number of boys, so we can use either formula to calculate $s_{12}$. The pooled standard deviation and the d-statistic value gives the value with which the two groups differ by. The measure $d$ is quite popular, because it is simple to use and makes good intuitive sense. If any measure can be called simply "the effect size", it is this. It is zero when there is no difference between the two means, and increases without bound when the difference becomes large. It is particularly useful in the field of the meta-analysis, which involves combining the findings from several studies. By working with d instead of the actual differences between means, the disparate studies are put on a common basis.

\subsubsection{The Proportion of Variability Accounted for by an Effect}

The d-statistic is a natural measure of effect size when only two groups are involved. When a study involves more than two groups, it is useful to have a single measure that summarizes the effect in the entire experiment. This measure is constructed by considering how the total variability is divided into systematic and unsystematic parts. 
The sum of the squares for the original set of data can be divided into a part associated with the difference between the groups and a part associated with error.

$$
S S_{T}=S S_{A}+S S_{S / A}
$$

The size of the differences among the groups determines $S S_{A}$, which is zero when the means in the experiment are all the same and positive when they are not. To obtain an overall measure of the effect, we can take the ratio of the size of this component to the total amount of variability:

$$
R^{2}=\frac{S S_{A}}{S S_{\text {TOTAL }}}=\frac{S S_{\text {TOTAL }}-S S_{S / A}}{S S_{\text {TOTAL }}}
$$

This quantity is known as the square of the correlation ratio. It is zero when there are no differences among groups and approaches as one as the group differences come to dominate the within-groups variability. An equivalent formula, based on the F-statistic, is

$$
R^{2}=\frac{(a-1) F}{(a-1) F+a(n-1)}
$$

This formula is often the easiest way to find $R^{2}$, particularly from published results which usually reports F's, not sums of squares. Effect-size measures of this type are widely reported. You may find it helpful to think of them in a broader context. The sums of squares are measures of the variability of the scores, so we can express the two parts of the equations above as

$$
\text { Effect Size }=\frac{\text { Variability Explained }}{\text { Total Variability }}=\frac{\text { Total Variability }- \text { Unexplained Variability }}{\text { Total Variability }}
$$


This expression is easily adapted to all kinds of treatment effects, including those in complex experimental designs and those based on procedures other than the analysis of variance.

In a very influential book on power analysis Cohen (1988) defined some standards for interpreting effect sizes

- $\quad$ Small Effect: is one that captures 1 percent of the variance (i.e. $R^{2}=0.01$ ). These effects tend not to be noticed except by statistical means. In terms of the standardized difference, small effect has $d \approx 0.25$.

- Medium Effect: captures about 6 percent of the variability (i.e. $R^{2}=0.06$ or $d \approx 0.5)$. These effects are apparent to careful observation, although they are not obvious to a casual glance.

- $\quad$ Large Effect: captures at least 15 percent of the variability (i.e. $R^{2}=0.15$ or $d \approx 0.8$ or more). It is obvious to superficial glance.

Using Cohen's guidelines, it is important to avoid any evaluative interpretation of the words "small", "medium" and "large". From a researcher's perspective, a large effect may not be better or more important than a small one. Indeed the type of the effects that need careful research and statistical analysis are the medium and small ones. Large effects, in Cohen's sense, are often widely known and there is nothing to be gained from verifying them. Where the discovery of large effects makes important contributions to psychological theory, they are often introduced and made possible by advances in instrumentation or the introduction of new paradigms instead of statistical analysis. Particularly in mature fields, the 
medium and small effects are the most often investigated. For example a survey by Sedlmeier and Gigerenzer (1989) reported that average study in the Journal of Abnormal Psychology produced a "medium" effect, a value that has been found in other areas of research (e.g. Cooper and Findley, 1982). There is some agreement among methodologists that a small effect is roughly the lower limit of what one might call a meaningful effect.

\subsection{Formulation of the Design Basis Equation}

Using the "correlation ratio" we can formulate the design basis equation for design engineers. Assuming that the total variability is $100 \%$ what we are going to determine how much contribution each independent variable makes towards the total variability. The total variability can be expressed as the percentage of the tracer gas. So the form of the Design Basis Equation would be like the given below:

$$
{ }^{0} \mathrm{CO}_{2_{\text {tot_var }}}=x U_{g}+y M_{s}+z R^{*}+a \zeta+b \varsigma
$$

The factors $x, y, z, a, b, c$ are the contributions of the independent variables towards the total variance. These factors will be determined for each independent variable using the correlation ratio discussed above. From the calculations shown in the appendix, we have the correlation ratios for the independent variables given in the table below.

\begin{tabular}{|c|c|}
\hline Independent Variable & Correlation Ratio \\
\hline $\mathrm{Ug}$ & 0.171 \\
\hline $\mathrm{Ms}$ & 0.189 \\
\hline
\end{tabular}




\begin{tabular}{|c|c|}
\hline $\mathrm{R}$ & 0.272 \\
\hline Axial Position & 0.178 \\
\hline Azimuthal & 0.085 \\
\hline
\end{tabular}

Table 6.5 Correlation Ratios for the Independent Variables

A pie chart showing the contributions of the independent variables towards the total variance is shown in figure 6.5

\section{Contribution of Each Independent Variable}

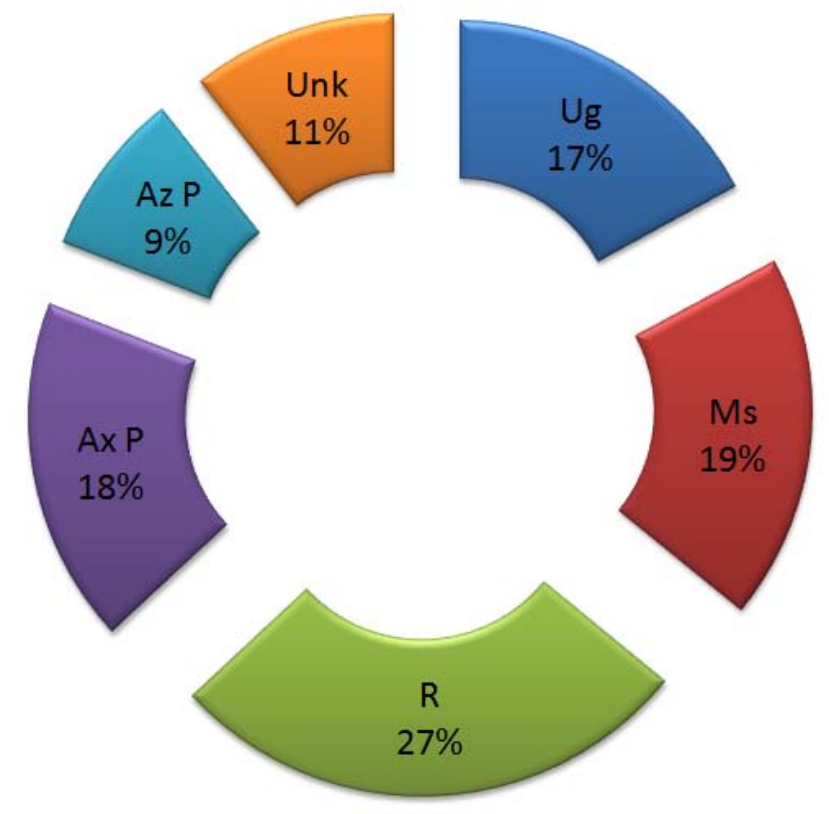

Fig 6.5 Pie chart showing contributions to total variance

As we can see from table 6.5 and from the pie chart, we can see that the superficial gas velocity $\left(\mathrm{U}_{\mathrm{g}}\right)$ and Solids Circulation Rates $\left(\mathrm{M}_{\mathrm{s}}\right)$ contribute equally to the total variance (17\%). The Radial position has the highest contribution (27\%) to the total variation. The azimuthal position has the least effect (9\%) on the total variance while the axial elevation has a slightly higher contribution (18\%) than the superficial gas velocity and solids circulation rates. Based on the division on the 
values of the correlation ratios we can clearly see that the effects of $U_{g}, M_{s}, R$, and $\zeta$ are large effects. The effect of azimuthal position $(\mathrm{S})$ is a medium effect while the contribution due to error or other independent variables is also a large effect.

Adding up the ratios we can explain $88 \%$ of the total variance based on the contributions of the above 5 independent variables. This means that there is there is a $12 \%$ of the total variance which is not attributed to the contributions from the five independent variables. This $12 \%$ of the total variance is attributed to the experimental, system and process error. It can also be attributed to the contribution to the total variance from other independent variables not taken into account in this study. Thus based on the correlation ratios we can come up with the design basis equation given below

$$
\% \mathrm{CO}_{2_{\text {total }} \text { variance }}=17 \mathrm{U}_{\mathrm{g}}+19 \mathrm{M}_{\mathrm{s}}+27 \mathrm{R}^{*}+18(\mathrm{Ax} . \text { Pos })+10(\text { Az. Pos })+12(\text { Unknown })
$$




\section{Chapter VII}

\section{Overall Conclusions and Future Recommendations \\ 7.1 Overall Conclusions}

This study investigates the gas and solids mixing process in the dense entry region of an asymmetrically loaded high density, high flux Circulating Fluidized Bed Riser. The experimental part of this study involves tracer gas measurements to obtain information on the gas and solids distribution in the dense entry region of the riser. A co-flow hypothesis has been proposed (Collaboratory for Multiphase Flow Research, Project 10009445, OSP Ref 06-485Z(New), 2006) where it is suggested that the tracer gas is injected with the solids the tracer gas gets entrapped within the solids. This co-flow condition exists only at the lower levels of the dense entry region before the tracer gas separates from the injected solids. Thus at these lower levels of the dense entry region, the hypothesis implies that the tracer gas concentration indicates the concentration of the injected solids at those elevations. However this technique is also used for determining the gas mixing at all elevations of the dense entry region of the riser. Based on the ANOVA analysis of the tracer gas data, this study proposes a statistical model that can be applied to determine which operational variables significantly affect the gas and solids mixing process. This information will be invaluable for design engineers and is also essential in developing and validating predictor reactor models

The gas tracer measurements show that there is a significant variation of the tracer gas concentration at different axial elevations for both azimuthal positions. This variation is more prominent for low solids circulation rates. The variation is not that significant for high solids circulation rates or for low solids and superficial gas flow rates. This effect is attributed to two factors.

The first factor is the solids momentum. As seen from the process description figures in chapters 3 and 4, for low solids circulation, the momentum of the solids is not sufficient to overcome the momentum of the dense mixing bed. As a result the 
solids with co-flowing tracer gas never reach the other side of the riser and hence we obtain a skewed profile. The skewness decreases as we go up in elevation and this decrease is evident from the tracer profiles. This phenomenon is observed for both azimuthal positions. For high solids circulation rates, the momentum of the incoming solids is quite sufficient to overcome the momentum of the superficial gas velocity and so the solids reach the opposite wall of the riser along with the coflowing tracer gas. This process is also observed for low solids circulation rates and low superficial gas velocity for now the low solids momentum is adequate to overcome the dense bed momentum. Here we do observe a skewed profile but the magnitude of skewness is not that severe. Also the variation or decrease in skewness as we go up in elevation is not drastic. This is true for both azimuthal positions. The tracer gas results were measured in the dense bed region of the riser for four axial elevations shown in figure 4.1. The dense bed transitioned from a packed to a turbulent bed as the superficial gas velocity increased with the test matrix evident from figure 4.6. The work done in this research differs from a majority of the contemporary work done. The test matrix for this work is in the four way coupling region shown in figure 1.2 whereas most of the work done is in the one way coupling region. It is believed that there were no core annular or dense suspension up-flow conditions existing in the dense region of the riser. However a core annular structure with DSU was observed for the same test conditions but at higher elevations beyond the scope of this research.

The second factor is called "Turbulence Modulation", where the presence of large particles and high Reynolds numbers tend to increase the turbulence on account of "Vortex Shedding". In addition, a dense particle suspension increases the turbulence as compared to a dilute particle suspension. This is because the vortex shedding increases for dense suspensions of large particles. In this study we have used large particles and the results we observe for low and high solids suspensions is attributed to turbulence modulation as explained in chapter $\mathrm{V}$.

In statistical analysis of this study, a Multivariate Analysis Of Variance (MANOVA) model was developed to analyze the data. The aim of this analysis is to 
determine which of the primary independent variables significantly influences the mixing process. We found that the almost all the primary independent variables listed in chapter 5 significantly influence the mixing process, in different orders of magnitude. It was also found that the variation in the operating conditions and the variation in axial elevations significantly influenced the mixing process for both azimuthal positions. Performing a comparative analysis on relative magnitudes of significance we showed how the significance of the variation in the operating conditions decreased with an increase in elevation. This coincides with the solids and gas moving from the dense entry region to the fully developed region of the riser where the latter is not influenced by changes in the operating conditions.

\subsection{Recommendation for Future Work}

The recommendations for future work are as follows

a. Develop a direct solids tracer technique to determine the solids concentration in the dense entry region of the riser. Having a direct measurement technique will help in corroborating the co-flow hypothesis and give credibility to the gas tracer technique as tool for measuring solids concentration in the lower levels of the dense entry region of the riser.

b. This study has focused on the dense entry region of the riser. The gas and solids mixing process in the fully developed and exit regions of the riser should also be investigated.

c. The existing feed section of the CFB riser needs modifications so that the solids can be fed from the bottom along with the superficial gas stream. A parametric study needs to be carried where solids are fed from the side and from the bottom in different ratios and the mixing process is studied for each case in all three regimes.

d. Further studies are needed to analyze the effect of turbulence modulation on the mixing process. Currently only one type B material is used. Use of different materials with different diameters and Reynolds number is needed to see how the turbulence modulation affects the mixing process.

e. Develop mathematical models for studying jet penetration into a dense bed. 
Chapter VII

Appendices 


\section{Appendix8.1}

Determination of the Confidence Interval for the Tracer Gas Measurements The measurements represented here are the 8 measurements made in the Riser Center The confidence interval determined for the riser center is assumed to hold true for all other radial points across the riser in both azimuthal directions.

mi - Measurement Index; all measurements are in ppm

Elevation - 0.762m

Azimuth - East West

Solids Feed - $2.835 \mathrm{~kg} / \mathrm{s}$

$\mathrm{m}_{1}:=404.6632$

$\mathrm{m}_{2}:=366.345$

$\mathrm{m}_{3}:=401.253$

$\mathrm{m}_{4}:=375.365$

$\mathrm{m}_{5}:=403.194$

$\mathrm{m}_{6}:=366.077$

$\mathrm{m}_{7}:=370.044$

$\mathrm{m}_{8}:=400.901$

mean_m $:=\frac{m_{1}+m_{2}+m_{3}+m_{4}+m_{5}+m_{6}+m_{7}+m_{8}}{8}$

mean_m $=385.98$

$\frac{\mathrm{m}_{1}-\text { mean_m }}{\mathrm{m}_{1}}=4.617 \% \quad\left|\frac{\mathrm{m}_{6}-\text { mean_m }}{\mathrm{m}_{6}}\right|=5.437 \%$

Thus we have a 95\% confidence and a 5\% error on the radial concentration values measured. 


\section{Appendix 8.2}

Average $\mathrm{CO} 2$ Calculations

These calculations are done to calculate the calculated mean CO2 ppm levels

$\mathrm{x}=\frac{\text { baseline_meas } 1+\text { baseline_meas } 2}{2}$

$\mathrm{x}:=\frac{555.271+490.571}{2}=522.921 \quad \mathrm{U}_{\mathrm{g}}:=25.649 \cdot 60 \cdot 60 \cdot 1 \cdot 1=9.234 \times 10^{4}$

THEORETICAL MEAN

$$
\begin{aligned}
& \frac{\mathrm{x} \cdot \mathrm{U}_{\mathrm{g}}}{6}+\mathrm{y} \cdot \mathrm{F}_{\mathrm{CO} 2} \quad \mathrm{~F}_{\mathrm{CO} 2}:=20 \quad \mathrm{y}:=1 \\
& \text { co2_calc }=\frac{10^{6}}{\mathrm{U}_{\mathrm{g}}+\mathrm{F}_{\mathrm{CO} 2}} \cdot 10^{6} \\
& \text { co2_calc }:=\frac{\frac{\mathrm{x} \cdot \mathrm{U}_{\mathrm{g}}}{10^{6}}+\mathrm{y} \cdot \mathrm{F}_{\mathrm{co} 2}}{\mathrm{U}_{\mathrm{g}}+\mathrm{F}_{\mathrm{CO} 2}} \cdot 10^{6}
\end{aligned}
$$$$
\text { co2_calc }=739.36 \quad \text { Calculated Mean }=739.36 \mathrm{ppm}
$$

\section{EXPERIMENTAL MEAN}

Ex_mean $:=\frac{1459.341+1176.516+957.407+857.184+506.532+698.026+567.532+557.448}{8}=847.498$

$\frac{\text { Ex_mean }- \text { co2_calc }}{\text { Ex_mean }}=12.76 \%$

Thus there is a $12 \%$ difference between the actual and theoretical mean. 


\section{Appendix 8.3}

F distribution critical value landmarks

Table entrles are critlcal values for $F^{*}$ with probably $p$ in right tal of the distribution.
Flgure of $F$ distribution (ilke in Moore, 2004, p. 656)

here.

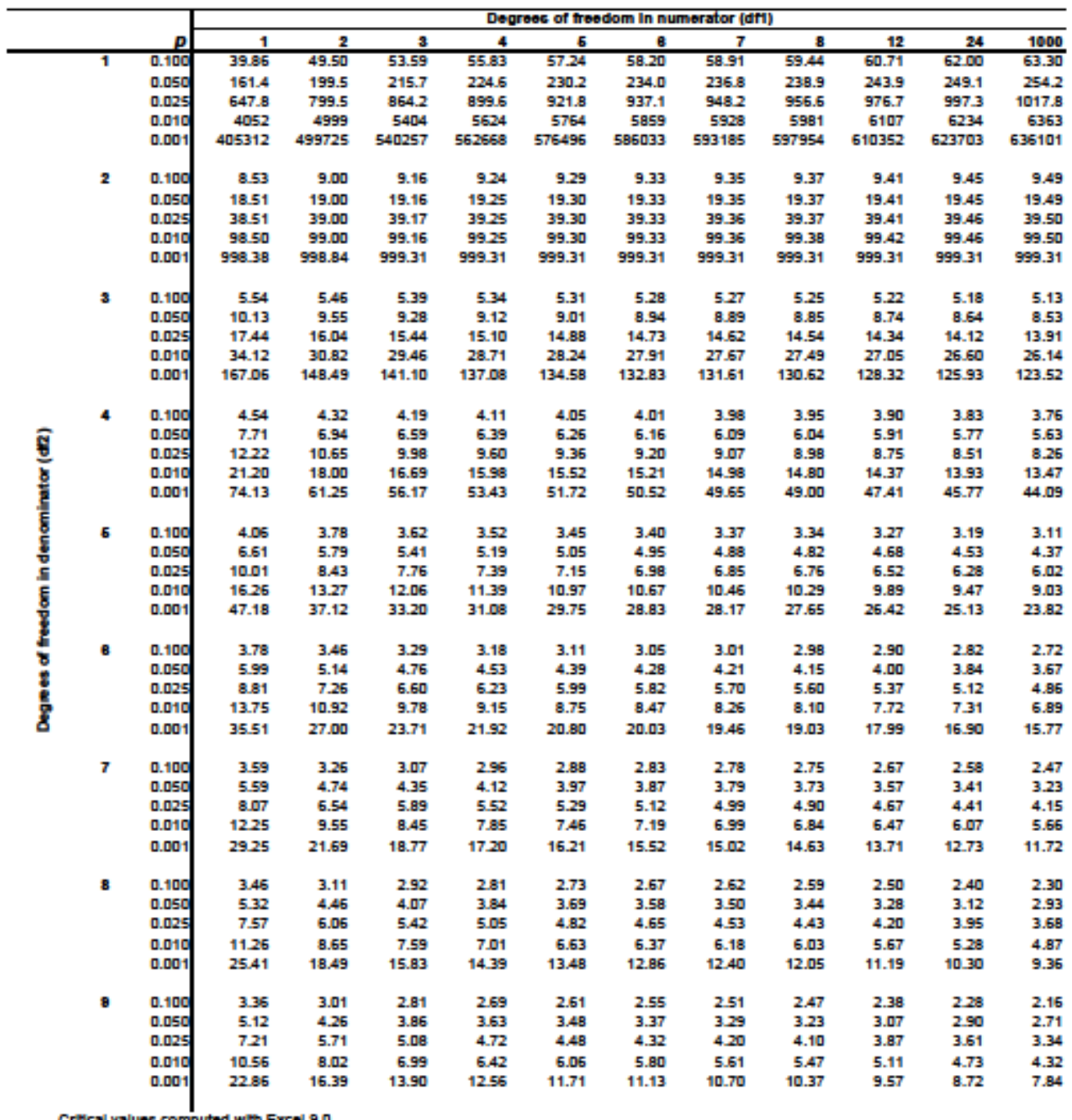

Critcal values computed with Excel 9.0 


\section{Appendix 8.4}

Correlation - Correlation measures the strength of the linear association between two variables. The formula for correlation (r) is:

$$
r=\frac{1}{n-1} \sum\left(\frac{x-\bar{x}}{s_{x}}\right)\left(\frac{y-\bar{y}}{s_{y}}\right)
$$

Computationally the Descriptor systems uses what is sometimes referred to as the sum of squares formula for $r$.

$$
r=\frac{\sum X Y-\frac{\sum X \sum Y}{N}}{\sqrt{\left(\sum X^{2}-\frac{\left(\sum X\right)^{2}}{N}\right)\left(\sum Y^{2}-\frac{\left(\sum Y\right)^{2}}{N}\right)}}
$$

Covariance - Covariance measures the extent to which pairs of points in two data series systematically vary around their respective means. In other words, if the paired values of $x$ and $y$ tend to be both be above or below the mean at the same time, then there will be a high positive covariance. If they tend to be on opposite sides of the mean then there will be a high negative covariance.

$$
\operatorname{Cov}_{x y}=\frac{\sum X Y-\frac{\sum X \sum Y}{N}}{N-1}
$$

Independence - Independence is a measure of the independent variation of each variable. It is calculated by regressing each variable on a constant and set of all other variables and then calculating the proportion unexplained (one minus r-squared).

Jarque-Bera - A statistic that measures the normality of an observed distribution. The J-B statistic is dependant on the values for Skewness and Kurtosis. J-B probability is calculated from chi-square table, with 2 degrees of freedom. J-B is not available for qualitative data if not ordered and numbered.

$$
J B=\frac{n}{6}\left(S k^{2}+\frac{(K-3)^{2}}{4}\right)
$$


Kurtosis - A measure of the data's flatness or peakedness. The Kurtosis of a "Normal” distribution is 3 . Kurtosis values greater than 3 indicate that the distribution is peaked relative to the normal (leptokurtic). If the Kurtosis is less than three the distribution is flatter than the ideal normal curve (platykurtic).

$$
K=\frac{n(n+1)}{(n-1)(n-2)(n-3)} \sum\left(\frac{x_{1}-\bar{x}}{s}\right)^{4}
$$

Mean - Arithmetic average, as a measure of concentration. It is sensitive to skewed data.

$$
\bar{x}=\frac{\sum x_{i}}{n}
$$

Median - The middle value of ranked observations if the number of observations is odd or mean of two middle observations if the number of observations is even. A measure of concentration that is insensitive to skewed data.

Minimum and Maximum - The smallest and largest observations of ranked observations.

Obs - Number of observations in a data series.

Skewness - A measure of the data's symmetry. If a distribution is "Normal" skewness will equal 0 (zero). A distribution with a significant positive skewness value has a long right tail. In other words there are one or more extreme large values.

$$
S k=\frac{1}{n} \sum\left(\frac{x_{i}-\bar{x}}{s}\right)^{3}
$$

Standard Deviation - A measure of how dispersed are the data.

$$
s=\sqrt{\frac{1}{n-1} \sum\left(x_{i}-\bar{x}\right)^{2}}
$$

Standardized Value - Sometimes called a z-score, a standardized value is a measure of how many standard deviations an observed values sits above or below the mean.

$$
z=\frac{x-\bar{x}}{s_{x}}
$$

\subsection{Critical Values for the F-Distribution $F(0.95)$}

F-Distribution (0.95) 


\begin{tabular}{|c|c|c|c|c|c|c|c|c|c|c|c|c|c|c|c|}
\hline \multirow{2}{*}{ DF } & \multicolumn{15}{|c|}{ DF Numerator } \\
\hline & 1 & 2 & 3 & 4 & 5 & 6 & 7 & 8 & 9 & 10 & 15 & 20 & 30 & 60 & 100 \\
\hline 1 & 161.45 & 199.50 & 215.71 & 224.58 & 230.16 & 233.99 & 236.77 & 238.88 & 240.54 & 241.88 & 245.95 & 248.02 & 250.10 & 252.20 & 253.04 \\
\hline 2 & 18.51 & 19.00 & 19.16 & 19.25 & 19.30 & 19.33 & 19.35 & 19.37 & 19.38 & 19.40 & 19.43 & 19.45 & 19.46 & 19.48 & 19.4 \\
\hline 3 & 10.13 & 9.55 & 9.28 & 9.12 & 9.01 & 8.94 & 8.89 & 8.85 & 8.81 & 8.79 & 8.70 & 8.66 & 8.62 & 8.57 & 8.5 \\
\hline 4 & 7.71 & 6.94 & 6.59 & 6.39 & 6.26 & 6.16 & 6.09 & 6.04 & 6.00 & 5.96 & 5.86 & 5.80 & 5.75 & 5.69 & 5.6 \\
\hline 5 & 6.61 & 5.79 & 5.41 & 5.19 & 5.05 & 4.95 & 4.88 & 4.82 & 4.77 & 4.74 & 4.62 & 4.56 & 4.50 & 4.43 & 4.41 \\
\hline 6 & 5.99 & 5.14 & 4.76 & 4.53 & 4.39 & 4.28 & 4.21 & 4.15 & 4.10 & 4.06 & 3.94 & 3.87 & 3.81 & 3.74 & 3.71 \\
\hline 7 & 5.59 & 4.74 & 4.35 & 4.12 & 3.97 & 3.87 & 3.79 & 3.73 & 3.68 & 3.64 & 3.51 & 3.44 & 3.38 & 3.30 & 3.2 \\
\hline 8 & 5.32 & 4.46 & 4.07 & 3.84 & 3.69 & 3.58 & 3.50 & 3.44 & 3.39 & 3.35 & 3.22 & 3.15 & 3.08 & 3.01 & 2.9 \\
\hline 9 & 5.12 & 4.26 & 3.86 & 3.63 & 3.48 & 3.37 & 3.29 & 3.23 & 3.18 & 3.14 & 3.01 & 2.94 & 2.86 & 2.79 & 2.7 \\
\hline 10 & 4.96 & 4.10 & 3.71 & 3.48 & 3.33 & 3.22 & 3.14 & 3.07 & 3.02 & 2.98 & 2.85 & 2.77 & 2.70 & 2.62 & .5 \\
\hline 11 & 4.84 & 3.98 & 3.59 & 3.36 & 3.20 & 3.09 & 3.01 & 2.95 & 2.90 & 2.85 & 2.72 & 2.65 & 2.57 & 2.49 & 2.4 \\
\hline 12 & 4.75 & 3.89 & 3.49 & 3.26 & 3.11 & 3.00 & 2.91 & 2.85 & 2.80 & 2.75 & 2.62 & 2.54 & 2.47 & 2.38 & 2.3 \\
\hline 13 & 4.67 & 3.81 & 3.41 & 3.18 & 3.03 & 2.92 & 2.83 & 2.77 & 2.71 & 2.67 & 2.53 & 2.46 & 2.38 & 2.30 & 2.2 \\
\hline 14 & 4.60 & 3.74 & 3.34 & 3.11 & 2.96 & 2.85 & 2.76 & 2.70 & 2.65 & 2.60 & 2.46 & 2.39 & 2.31 & 2.22 & 2.1 \\
\hline 15 & 4.54 & 3.68 & 3.29 & 3.06 & 2.90 & 2.79 & 2.71 & 2.64 & 2.59 & 2.54 & 2.40 & 2.33 & 2.25 & 2.16 & 2.12 \\
\hline 16 & 1.49 & 3.63 & 3.24 & 3.01 & 2.85 & 2.74 & 2.66 & 2.59 & 2.54 & 2.49 & 2.35 & 2.28 & 2.19 & 2.11 & 2.07 \\
\hline 17 & 4.45 & 3.59 & 3.20 & 2.96 & 2.81 & 2.70 & 2.61 & 2.55 & 2.49 & 2.45 & 2.31 & 2.23 & 2.15 & 2.06 & 2.0 \\
\hline 18 & 4.41 & 3.55 & 3.16 & 2.93 & 2.77 & 2.66 & 2.58 & 2.51 & 2.46 & 2.41 & 2.27 & 2.19 & 2.11 & 2.02 & 1.9 \\
\hline 19 & 4.38 & 3.52 & 3.13 & 2.90 & 2.74 & 2.63 & 2.54 & 2.48 & 2.42 & 2.38 & 2.23 & 2.16 & 2.07 & 1.98 & 1.9 \\
\hline 20 & 4.35 & 3.49 & 3.10 & 2.87 & 2.71 & 2.60 & 2.51 & 2.45 & 2.39 & 2.35 & 2.20 & 2.12 & 2.04 & 1.95 & 1.9 \\
\hline 21 & 4.32 & 3.47 & 3.07 & 2.84 & 2.68 & 2.57 & 2.49 & 2.42 & 2.37 & 2.32 & 2.18 & 2.10 & 2.01 & 1.92 & 1.8 \\
\hline 22 & 4.30 & 3.44 & 3.05 & 2.82 & 2.66 & 2.55 & 2.46 & 2.40 & 2.34 & 2.30 & 2.15 & 2.07 & 1.98 & 1.89 & 1.8 \\
\hline 23 & 4.28 & 3.42 & 3.03 & 2.80 & 2.64 & 2.53 & 2.44 & 2.37 & 2.32 & 2.27 & 2.13 & 2.05 & 1.96 & 1.86 & 1.8 \\
\hline 24 & 4.26 & 3.40 & 3.01 & 2.78 & 2.62 & 2.51 & 2.42 & 2.36 & 2.30 & 2.25 & 2.11 & 2.03 & 1.94 & 1.84 & 1.8 \\
\hline 25 & 4.24 & 3.39 & 2.99 & 2.76 & 2.60 & 2.49 & 2.40 & 2.34 & 2.28 & 2.24 & 2.09 & 2.01 & 1.92 & 1.82 & 1.7 \\
\hline 26 & 4.23 & 3.37 & 2.98 & 2.74 & 2.59 & 2.47 & 2.39 & 2.32 & 2.27 & 2.22 & 2.07 & 1.99 & 1.90 & 1.80 & 1.76 \\
\hline 27 & 4.21 & 3.35 & 2.96 & 2.73 & 2.57 & 2.46 & 2.37 & 2.31 & 2.25 & 2.20 & 2.06 & 1.97 & 1.88 & 1.79 & 1.7 \\
\hline 28 & 4.20 & 3.34 & 2.95 & 2.71 & 2.56 & 2.45 & 2.36 & 2.29 & 2.24 & 2.19 & 2.04 & 1.96 & 1.87 & 1.77 & 1.73 \\
\hline 29 & 4.18 & 3.33 & 2.93 & 2.70 & 2.55 & 2.43 & 2.35 & 2.28 & 2.22 & 2.18 & 2.03 & 1.94 & 1.85 & 1.75 & 1.71 \\
\hline 30 & 4.17 & 3.32 & 2.92 & 2.69 & 2.53 & 2.42 & 2.33 & 2.27 & 2.21 & 2.16 & 2.01 & 1.93 & 1.84 & 1.74 & 1.7 \\
\hline 60 & 4.00 & 3.15 & 2.76 & 2.53 & 2.37 & 2.25 & 2.17 & 2.10 & 2.04 & 1.99 & 1.84 & 1.75 & 1.65 & 1.53 & 1.4 \\
\hline 100 & 3.94 & 3.09 & 2.70 & 2.46 & 2.31 & 2.19 & 2.10 & 2.03 & 1.97 & 1.93 & 1.77 & 1.68 & 1.57 & 1.45 & 1.3 \\
\hline 1000 & 3.85 & 3.00 & 2.61 & 2.38 & 2.22 & 2.11 & 2.02 & 1.95 & 1.89 & 1.84 & 1.68 & 1.58 & 1.47 & 1.33 & \\
\hline
\end{tabular}


$4 \quad$ Definitions of ANOVA Parameters

$\underline{\text { SUM OF SQUARES }}\left(\sum \square\right.$ 's)

Total Sum of Squares - Summation of the square of the dependent variables ( $\left.Y_{\exp }\right)$.

Model Sum of Squares - Sum of squares accounted for by the model.

Residual Sum of Squares - Sum of squares not accounted for by the model.

Sum of Squares for 'Left Out Terms' - Sum of squares contributed to the residual due to the lack of fit of the model with the experimental data.

True Error Sum of Squares - Sum of squares contributed to the residual that would be expected due to sampling error for the number of total samples taken.

DEGREES OF FREEDOM $\quad\left({ }^{\circ}\right.$ Freedom )

Total Degrees of Freedom - total number of samples taken.

Model Degrees of Freedom - the number of adjustable parameters in the model.

Residual Degrees of Freedom - The difference between the total and model degrees of freedom. This is the number of degrees of freedom that are not taken up by the model.

Degrees of Freedom for 'Left Out Terms' - The 'left over' degrees of freedom that could be used to add additional terms / parameters to the model.

True Error Degrees of Freedom - The number of degrees of freedom that are utilized in estimating the true error of the system. This is equal to the number of independent estimates of the error, which is equal to the number of sample levels that have independent replicates.

\section{MEAN SQUARE $\quad($ Mean $\square)$}

Model Mean Square - The average square of the dependent variable associated with each degree of freedom of the model; equal to the model sum of squares divided by the number of degrees of freedom of the model.

Mean Square for 'Left Out Terms' - The average square associated with each degree of freedom for those terms / parameters not included in the model.

True Error Mean Square - The average square error as calculated from multiple estimates of the error from replicates at different sample levels.

\section{CALCULATED 'F TEST' RATIO (F calculated)}

Calculated F ratio for Model - Ratio of the mean square of the model to the mean square of the true error. A good model will have a high mean square for the model; therefore, the larger this ratio, the better the model 'fits' the experimental data. 
Calculated F ratio for 'Left Out Terms' - Ratio of the mean square of the 'Left Out Terms' to the mean square of the true error. A good model will have a low mean square for the 'Left Out Terms'; therefore, the smaller this ratio, the better the model 'fits' the experimental data.

Tabulated 'F Test' Ratio (F table )

Model 'F test' ratio - $\mathrm{F}$ ratio is taken from the table based on the number of degrees of freedom of the model (numerator - typically x coordinate of $\mathrm{F}$ table) and the number of degrees of freedom for the error (denominator - typically the y coordinate of the $\mathrm{F}$ table).

'F test' ratio for 'Left Out Terms' - F ratio is taken from the table based on the number of degrees of freedom of the 'Left Out Terms' (numerator - typically x coordinate of F table) and the number of degrees of freedom for the error (denominator - typically the y coordinate of the F table). 
II. Sequential Procedure used to Generate an ANOVA Table

Filling in an ANOVA table is completed in two steps. First, parts of the table are computed from the experimental data. The second step involves calculating the remaining parts of the table from definitions which interrelate various parts of the table. This allows the calculation of $F$ ratios which can be used to test the statistical significance of the proposed model, and possible 'Left Out Terms'.

\subsection{Step 1 - Calculation of Table Entries from Experimental Data}

Calculate total sum of squares - sum square of all points

$$
\text { Total Sum of Squares }=\sum_{i=1}^{n}\left(Y_{\text {exp erimental }}\right)^{2}
$$

Evaluate the model sum of squares - determined by the minimum square error determined from the computer program.

$$
\text { Model Sum of Squares }=\sum_{i=1}^{n}\left(\mathbf{Y}_{\text {mod el }_{\mathrm{i}}}\right)^{2}
$$

\section{Estimate True error sum of squares}

calculated from the variance of replicates, $\mathrm{p}_{\mathrm{j}}=$ \# of replicates at $\mathrm{j}^{\text {th }}$ level sum the variance of replicates at each experimentallevel, $\mathrm{m}=\#$ of levels

True Error Sum of Squares $=\sum_{j=1}^{m} \frac{\sum_{\mathrm{k}=1}^{\mathrm{p}}\left(\mathrm{Y}_{\text {exp erimental }}-\overline{\mathrm{Y}_{\mathrm{avg}_{\mathrm{j}}}}\right)^{2}}{\mathrm{P}_{\mathrm{j}}-1}$

The value of the true error sum of squares is actually the sum of the squared variance at each of the experimental levels. In terms of setting up a spreadsheet, this may be an easier way of calculating it. [sum (STD) ${ }^{2}$ for all ' $m$ ' experimental levels]

where the average at experimental level $j$ is: $\overline{Y_{\text {avg }}}=\frac{1}{P_{j}} \cdot \sum_{j=1}^{P_{j}} Y_{\text {exp erimental }_{k}}$

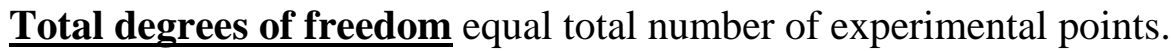

Model degrees of freedom equal the number of adjustable parameters in the model.

Error degrees of freedom equal the number of independent estimates of error.

(equal to the number of experimental levels with replicates) 
5 Step 2 - Calculate the Rest of the Table as Dependent Variables from Step 1

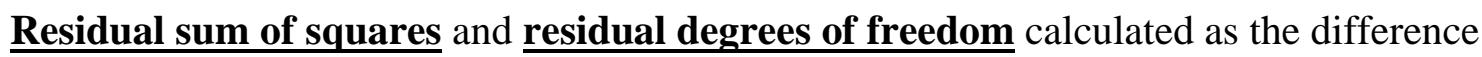
between respective total and model values.

$$
\text { Residual }=\text { Total }- \text { Model }
$$

'Left Out Terms' sum of squares and 'Left Out Terms' degrees of freedom calculated as the difference between respective residual and error values.

'Left Out Terms’ $(\mathrm{LOT})=$ Residual - Error

The mean square for model, 'Left Out Terms', and Error are calculated as the ratio of the respective sum of squares divided by the corresponding degrees of freedom. Example:

$$
(\text { Mean Square })_{\text {model }}=\frac{(\text { Sum of Squares })_{\text {model }}}{(\text { Degrees of Freedom })_{\text {mod el }}}
$$

F ratio for model is the ratio of the mean square of the model to the mean square of the error.

$$
(\text { F calculated })_{\text {model }}=\frac{(\text { Mean Square })_{\text {mod el }}}{(\text { Mean Square })_{\text {true error }}}
$$

F ratio for 'Left Out Terms' is the ratio of the mean square of the 'Left Out Terms' to the mean square of the error.

$$
(\text { F calculated }) \text { "left out terms” }=\frac{(\text { Mean Square })_{\text {"left out terms" }}}{(\text { Mean Square })_{\text {true error }}}
$$




\section{Chapter IX \\ References}

Abrahamsen, A.R., Gcldart, D., Powder Technology, vol. 26, p. 35, 1980.

Adams, C.K., "Gas Mixing in Fast Fluidized Bed", in Circulating Fluidized Bed

Technology II, ed., Basu, P,. Large, J.F., Pergamon Press, Toronto, pp. 299-306, 1988.

Amos, G., Rhodes, M.J., Mineo H., "Gas Mixing in Gas-Solid Risers", Chemical Engineering Science, vol. 48, No. 5, pp. 943-949, 1993.

Arena, U., "Gas Mixing" in Circulating Fluidized Beds, eds., Grace, J.R., Avidan, A.A., Knowlton, T.M., chp. 3, pp. 86-1 18, Chapman \& Hall, London, 1997.

Arena, U., A. Cammarota, and L. Pistone, "High Velocity Fluidization Behavior of Solids in a Laboratory Scale Circulating Bed," Circulating Fluidized Bed Technology I, P. Basu, ed., Pergamon Press, Oxford, p. 19 (1985).

Arena, U., Marzocchella, A., Massimilla, L., Malandrino, A., "Hydrodynamics of Circulating Fluidized Beds with Risers of Different Shape and Size", Powder Technology, 70,237-247, 1992.

Bai, D.-R., Jin, Y., and Yu, 2.-Q. (1990), 'Particle Acceleration Length in a Circulating Fluidized Bed", Chem. Eng. and Technol. (in Chinese), 34-39.

Bai, D.R., Jin, Y., Yu, Z.Q. and Gan, N.J. (1991), "Radial Profiles of Local Solids Concentration and Velocity in a Concurrent Downflow Fast Fluidized Bed", in Circulating Fluidized Bed Technology ID, P. Basu, M. Horio and M. Hasatani, eds., pergamon Press, Toronto, pp. 157- 162. 
Bai, D.-R., Jin, Y., Yu, 2.-Q. and Zhu, J.-X. (1992), "The Axial Distribution of the Cross- Sectionally Averaged Voidage in Fast Fluidized Beds", Powder Technol., 71.5 $1-58$

Bai, D.-R., Jin, Y. and Yu, Z. (1993), "Flow Regimes in Circulating Fluidized Beds", Chem. Eng. Technol., 16.307-313.

Bai, D.-R. and Kato, K. (1 995), "Saturation Carrying Capacity of Gas and Flow Regimes in CFB", J. Chem. Eng. Japan, 28, 179-185.

Bai, D., Yi, J., Jin, Y., Yu, Z., "Residence Time Distributions of Gas and Solids in a Circulating Fluidized Bed", in Fluidization VII, eds., Potter, O.E., Nicklin, D.J., Engineering Foundation, pp. 195-202, 1993.

Beruti, F., Chaouki, J., Pugsley, T.S., Patience, G.S., "Hydrodynamics of Circulating Fluidized Bed Risers: A Review", the Canadian Journal of Chemical Engineering, vol. 73, p. 579-602, 1995.

Bi, H. T., and L.S. Fan, "Existence of turbulent regime in gas-solid fluidization," AIChE J., 38, 297 (1992).

Bi, H.T., and J.R. Grace, "Effect of measurement method on the velocities used to demarcate the onset of turbulent fluidization," Chem. Eng. J., 57, 251 (1995a).

Bi, H.T., and J.R. Grace, "Flow regime diagrams for gas-solid fluidization and upward transport," Int. J. Multiphase Flow, 21 (6), 1229 (1995b).

Bi, H.T., and L.-S. Fan, "Regime transition in gas-solid circulating fluidized beds," AIChE Annual Meeting, Los Angeles, Calif., Nov. 17 (1991). 
Bi, H.T., and P.C. Su, "Local Phase holdups in gas-solids fluidization and transport," AIChE J., 47 (9), 2025 (2001).

Bi, H.T., J.R. Grace, and J.X. Zhu, "Regime transitions affecting gas-solids suspensions and fluidized beds," Trans. IChemE., 73, 154 (1995).

Bi, H.T., J.R. Grace, and J.X. Zhu, "Types of choking in vertical pneumatic systems,"

Int. J. Multiphase Flow, 19 (6), 1077 (1993).

Bi, H.T., N. Ellis, I.A. Abba, and J.R. Grace, "A state-of-the-art review of gas-solid turbulent fluidization," Chem. Eng. Sci., 55, 4789 (2000).

Brereton, C., Grace, J.R., "End Effects in Circulating Fluidized Bed Hydrodynamics", in Preprints of CFB IV, ed., Avidan, A.A., pp. 169- 174, 1993.

Elgobashi, S., "On Predicting Particle-Laden Turbulent Flows", Applied Scientific Research, vol. 52, p. 309-329, 1994.

Elgobashi, S.E.. Abou-Arab, T.W., "A Two-Equation Turbulence Model for TwoPhase Flows", Physics of Fluids, vol- 26(4), p. 931-938, 1983.

Ergun, S., Fluid flow through packed columns, Chem. Eng. Prog. 48 (1952) 89- 94.

Gayan, P., Diego, L.F., Adanez. D.J.,"Radial Gas Mixing in a Fast Fluidized Bed", Powder Technology, vol. 94, pp. 163- 171, 1997.

Gore, R.A., Crowe, C.T., "Effect of Particle Size on Modulating Turbulent Intensity", Int. J. Multiphase Flow, vol. 15, no. 2, p. 279-285, 1989. 
Grace, J.R. (1990), "High Velocity Fluidized Bed Reactors", Chem. Eng. Sci., $45,1953-1966$.

Grace, J.R., Bi, KT., "Fundamentals of Circulating Fluidized Beds", in Circulating Fluidized Beds, eds., Grace, J.R., Avidan, A.A., Knowlton, T.M., chp. 1, Chapman \& Hail, London, 1997.

Grace, J.R., Issangya, A.S., Bai, D., Bi, H.. Zhu, J., "Situating the High-Density Circulating Fluidized Bed", AiChE J, vol. 45, pp. 2108-2116, 1999.

Hestroni, G., "Particles-Turbulence Interaction". Int. J. Multiphase Flow, vol. 15, No. 5, pp. 735-746, 1989.

Hirama, T., H. Takeuchi, and T. Chiba, "Regime Classification of Macroscopic GasSolid Flow in a Circulating Fluidized Bed Riser," Powder Technol., 70, 215 (1992).

Horio, M., "Hydrodynamics", in Circulating Fluidized Beds, eds., Grace, J.R., Avidan, A.A., Knowlton, T.M., chp. 3, Chapman \& Hall, London. 1997.

Horio, M., Ishii, H., Nishimuro, M., "On the Nature of Turbulent and Fast Fluidized Beds", Powder technology, vol. 70, p. 229-236, 1992.

Hrenya, C.M., Sinclair, J.L., "Effects of Particle-Phase Turbulence in Gas-Solid Flows", AIChE J, vol. 43, no. 4., pp. 853-869, 1997.

Johnsson, F., Andersson, S. \& Leckner, B. 1991 Expansion of a freely bubbling bed. Powder Technol. 68, 117-123. 
Johnsson, F. \& Leckner, B. 1995 Vertical distribution of solids in a CFB furnace. In Proe. 13 $3^{\text {th }}$ Conf. Fluidized Bed Combustion (Edited by Heinschel, K. J.). The American Society of Mechanical Engineers, New York, pp. 671-679.

Johnsson, F., Svensson, A. \& Leckner, B. 1992 Fluidization regimes in circulating fluidized bed boilers. In Fluidization VII (Edited by Potter, O. P. \& Nicklin, D. J.). Engineering Foundation, pp. 471--478.

Kim, S.W., Kirbas, G., Bi, H., Lim, J.L., Grace, J. R., "Flow behavior and regime transition in a high-density circulating fluidized bed riser". Chemical Engineering Science, 59 (18), pp. 3955-3963.

Knowlton, T.M., Bachovchin, D.M., "The determination of gas-solids pressure drop and choking velocity as a function of gas density in a vertical pneumatic conveying line”, in: D.L. Keairns (Ed.), Fluidization Technology, Hemisphere Publishing Corp, Washington D.C., 1976, pp. 253- 282.

Kunii, O., Levenspiel, O., "Circulating Fluidized Bed-Reactors", Chemical Engineering Science, vol. 52, No. 15, p. 2471 -2482, 1997.

Kunii, D., Levenspiel, O., "Effect of Exit Geometry on the Vertical Distribution of Solids in Circulating Fluidized Beds. Part 1: Solution of Fundamental Equations; Part II: Analysis of Reported Data and Prediction", Powder Technology, 84, 83-90, (1995).

Kunii, D., Levenspiel, O., Fluidization Engineering, Butterworth-Heinemann, 1991.

Kwauk, M., and J. Li, “Fluidization Regimes,” Powder Technol., 87-193 (1996). 
Leckner, B., "Fluidized Bed Combustion: Mixing and Pollutant Limitation", Progress in Energy and Combustion Science, vol. 24, pp. 3 1-6 1, 1998.

Leung L.S., Wiles, R.J., Nicklin, D.J., "Correlation for predicting choking flowrates in vertical pneumatic conveying”, Ind. Eng. Chem. Process Des. Dev. 10 (1971) 183189.

Levenspiel. O., Chemical Reaction Engineering, Wiley \& Sons, 2nd Edition, 1964.

Li, Y., Wu, P., "A Study on Axial Gas Mixing in a Fast Fluidized Bed", in Circulating Fluidized Bed Technology III, eds., Bassu, P., Horio, M., Hasatani, M., Pergamon Press, Toronto, pp. 581-586, 1991.

Lim. K.S.. Zhu. J.X., Grace, J.R., "Hydrodynamics of Gas-Solid Fluidization", Int. J. Multipiiasc Flow, vol. 2 1, Suppl., pp. 141- 193. 1995.

Liu, J., Grace, J.R., Bi, H., Morikowa, H., Zhu, J., "Gas dispersion in fast fluidization and dense suspension up flow", Chem. Eng. Sci., vol. 54, pp. 5441-5449, 1999.

Namkung, W., Kim, S.D., "Radial Gas Mixing in a Circulating Fluidized Bed", Powder Technology, vol. 113, 23-29, 2000.

Patience, G.S., Chaouki, J., "Gas Phase Hydrodynamics in the Riser of a Circulating Fluidized Bed", Chemical Engineering Science, vol. 48, No. 18, pp. 3 195-3205, 1993.

Peirano, E.. Leckner, B., "Fundamentals of Turbulent Gas-Solid Flows Applied to Circulating Fluidized Bed Combustion", Progress in Energy and Combustion Science, vol. 24, pp. 359-296, 1998. 
Peirano, E., Palchonok, G.I., Johnsson, F., Leckner, B.,"Estimates of Turbulence Mechanisms in Circulating Fluidized Bed Combustors" Powder Technology, vol. 96, pp. 90-105, 1998.

Pugsley, T., Lapointe, D., Hirschberg, B., Werther, J., "Exit Effects in Circulating Fluidized Bed Risers", Can. J. Chem. Eng., 75, 1001 - 10 10, 1997.

Reh, L. and J. Li (1991), "Measurement of Voidage in Fluidized Beds by Optical Probes", in Circulating Fluidized Bed Technology III, P. Basu, M. Horio, and M. Hasatani, Eds., Pergamon Press, Toronto, pp. 163- 170.

Rhodes, M., "What is Turbulent Fluidization?" Powder Technology, vol. 88, pp. 3-14, 1996.

Sinclair, J.L., Jackson, R., "Gas-Particle Flow in a Vertical Pipe with ParticleParticle Interactions", AIChE Journal, vol. 35, no. 9, pp. 1473-1486, 1989.

Sterneus, J., Johnsson, F., Leckner, B., "Gas Mixing in Circulating Fluidised-Bed Risers", Chem. Eng. Science, vol. 55, pp. 129-148, 2000.

Sterneus, J., Johnsson, F., Leckner, B., Palchonok, G.I., "Gas and Solids Flow in Circulating Beds - Discussion on Turbulence", Chem. Eng. Science, vol. 54, pp. 5377-5382, 1999.

Svensson, A., Johnsson, F. \& Leckner, B. 1993 Fluid-dynamics of the bottom bed of circulating fluidized bed boilers. Proc. 12th Int. Conf. on Fluidized Bed Combustion (Edited by Rubow, L. N. \& Commonwealth, G.). The American Society of Mechanical Engineers, New York, pp. 887-897. 
Svensson, A., Johnsson, F. \& Leckner, B. 1996 Fluidization regimes in non-slugging fluidized beds: the influence of pressure drop across the air distributor. Powder Technol. 68, 299-312.

Takeuchi, H., T. Hirama, and T. Chiba, "A Quantitative Definition and Flow Regime Diagram for Fast Fluidization,” Powder Technol., 47, 195 (1986).

van Deemter, J.J., "Mixing Patterns in Large-Scale Fluidized Beds", in Fluidization, eds., Grace, J.R. and Matsen, J.M., Plenum Press, New York, 1980.

van Deemter, J.J., "Mixing" in Fluidization, eds., Davidson, J.F., Clift, R., Harrison, D., $2^{\text {nd }}$ Edition, Academic Press, 1985.

Weinstein, H., and J. Li, "An Evaluation of the Actual Density in the Acceleration Section of Vertical Risers," Powder Technol., 57-77 (1989).

Werther, J., "Fluid Mechanics of Large-Scale CFB Units", Circulating Fluidized Bed Technology IV, Ed., Avidan, A.A., pp. 1- 14, AIChE, New York, 1994.

Werther, J., Hartge, E.U., Kruse, M., "Radial Gas Mixing in the Upper Dilute Core of a Circulating Fluidized Bed", Powder Technology, vol. 70, p. 293-301, I992a.

Werther, J., Hartge, E.U ., Kmse, M., Nowak, W., "Radial Gas Mixing in Core Zone of a Pilot Scale CFB", in Circulating Fluidized Bed Technology III, ed., Basu, P., Horio, M., Hasatani, M., Pergamon Press, Toronto, pp. 593-598, 1991.

Werther, J., Hartge, E.U., Kruse, M., "Gas Mixing and Interphase Mass Transfer in the Circulating Fluidized Bed", in Fluidization VII, eds., Potter, O.E., Nicklin, D.J., Engineering Foundation, pp. 257-264, 1992b. 
Werther, J., Hirschberg, B., "Factors Affecting Segregation in Circulating FluidizedBed Riser", AIChE J, vol. 44, no.I, pp. 25-34, 1998.

Schlichthaerle, P., Werther, J., "Solids Mixing in the bottom zone of a circulating fluidized bed". Power Technology 120, pp 21-33, 2001.

Yang, W. C., ““'Choking” revisited,” Ind. Eng. Chem. Res., 43, 5496 (2004).

Yang, W.C., "A mathematical definition of choking phenomenon and a mathematic model for predicting choking velocity and choking voidage," AIChE J., 21 (5), 1013 (1975).

Yang, W. C., "Criteria for choking in vertical pneumatic conveying lines", Powder Technol. 35 (1983) 143- 150.

Yates, J., Simons, S.J.R, "Experimental Methods in Fluidization Research", Int. J. Multiphase Flow, vol. 20, Suppl., p. 297-330, 1994.

Yerushalmi, Y., and Avidan A.A., "High Velocity Fluidization" in Fluidization (J. F Davidson, R. Clift and D. Harrison, eds.), 2 ${ }^{\text {nd }}$ edition, Academic Press, New York (1995)

Y. Yousfi, G. Gau, Chem. Eng. Sci. 29 (1974) 1939-1946.

Zenz F.A., and Othmer D. F., "Fluidization and Fluid Particle Systems" Reinhold Publishing Corporation, New York, 1960.

Zheng, Q., Wei, X., Fei. L., "Experimental Study on Radial Gas Dispersion and its enhancement in Circulating Fluidized Beds", in Fluidization VII, eds., Potter. O.E., Nicklin, D.J., Engineering Foundation, pp. 285-293, 1992. 
Zhou, J., Grace, J.R., Brereton, C.H.M.. Lim, C.J.. "Influence of Wall Roughness on the Hydrodynamics in a Circulating Fluidized Bed". AIChE J., 42, no.4, 1 153-1 156, 1996.

Zhu, J.X., Bi, H.T., "Development of CFB and High Density Circulating Fluidized Beds". in Preprints of CFB IV. ed., Avidan, A.A., pp. 565-570. 1993. 
GAS-SOLID INTERACTION AND FLOW BEHAVIOR ANALYSIS IN THE DENSE ENTRY REGION OF AN ASYMMETRICALLY LOADED COLD

FLOW CFB RISER

Rajiv Dastane

Thesis submitted

to

The College of Engineering and Mineral Resources

at West Virginia University

in partial fulfillment of the requirements for the degree of

\author{
Master of Science \\ in \\ Aerospace Engineering
}

Department of Mechanical and Aerospace Engineering

APPROVAL OF THE EXAMINING COMMITTEE

Bruce S. Kang, Ph.D.

Lawrence J. Shadle, Ph.D.

Date

Eric K. Johnson, Ph.D., Chair

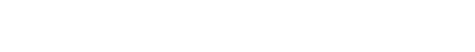

